\title{
Depletion Analysis of Modular High Temperature Gas-Cooled Reactor Loaded with LEU/Thorium Fuel
}

Sonat Sen Gilles Youinou

February 2013

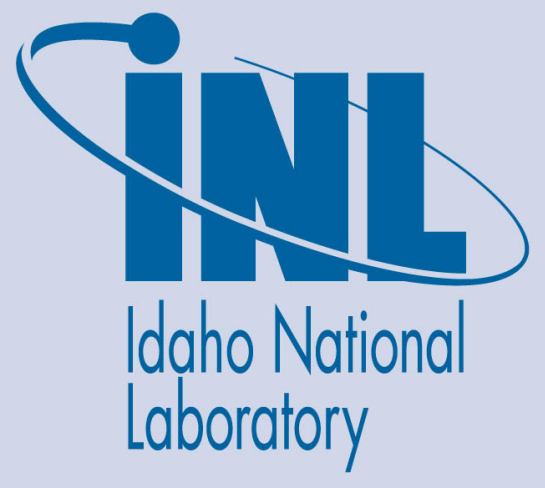

The INL is a U.S. Department of Energy National Laboratory operated by Battelle Energy Alliance 
INL/EXT-13-28449

FCRD-FCT-2013-000105

\title{
Depletion Analysis of Modular High Temperature Gas- Cooled Reactor Loaded with LEU/Thorium Fuel
}

\author{
Sonat Sen \\ Gilles Youinou
}

February 2013

\author{
Idaho National Laboratory \\ Fuel Cycle Research and Development \\ Idaho Falls, Idaho 83415
}

http://www.inl.gov

Prepared for the

U.S. Department of Energy

Office of Nuclear Energy

Under DOE Idaho Operations Office

Contract DE-AC07-05ID14517 


\section{DISCLAIMER}

This information was prepared as an account of work sponsored by an agency of the U.S. Government. Neither the U.S. Government nor any agency thereof, nor any of their employees, makes any warranty, expressed or implied, or assumes any legal liability or responsibility for the accuracy, completeness, or usefulness, of any information, apparatus, product, or process disclosed, or represents that its use would not infringe privately owned rights. References herein to any specific commercial product, process, or service by trade name, trade mark, manufacturer, or otherwise, does not necessarily constitute or imply its endorsement, recommendation, or favoring by the U.S. Government or any agency thereof. The views and opinions of authors expressed herein do not necessarily state or reflect those of the U.S. Government or any agency thereof. 
Depletion Analysis of Modular High Temperature Gas-cooled Reactor Loaded with LEU/Thorium Fuel

February 2013 
Depletion Analysis of Modular High Temperature Gas-cooled Reactor Loaded with LEU/Thorium Fuel 


\section{SUMMARY}

Thorium based fuel has been considered as an option to uranium-based fuel, based on considerations of resource utilization (Thorium is more widely available when compared to Uranium). The fertile isotope of Thorium (Th-232) can be converted to fissile isotope U-233 by neutron capture during the operation of a suitable nuclear reactor such as High Temperature Gas-cooled Reactor (HTGR). However, the fertile Thorium needs a fissile supporter to start and maintain the conversion process such as U-235 or Pu-239.

This report presents the results of a study that analyzed the thorium utilization in a prismatic HTGR, namely Modular High Temperature Gas-Cooled Reactor (MHTGR) that was designed by General Atomics (GA). The collected for the modeling of this design come from Chapter 4 of MHTGR Preliminary Safety Information Document that GA sent to Department of Energy (DOE) on 1995.

Both full core and unit cell models were used to perform this analysis using SCALE 6.1 and Serpent 1.1.18. Because of the long mean free paths (and migration lengths) of neutrons in HTRs, using a unit cell to represent a whole core can be non-trivial. The sizes of these cells were set to match the spectral index between unit cell and full core domains. It was found that for the purposes of this study an adjusted unit cell model is adequate.

Discharge isotopics and one-group cross-sections were delivered to the transmutation analysis team. This report provides documentation for these calculations. 
Depletion Analysis of Modular High Temperature Gas-cooled Reactor Loaded with LEU/Thorium Fuel 


\section{CONTENTS}

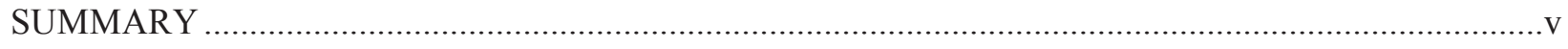

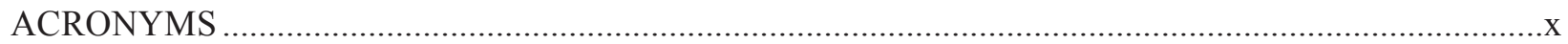

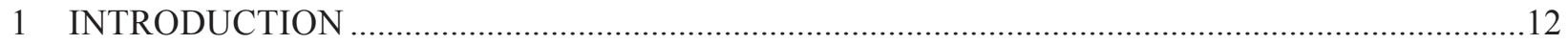

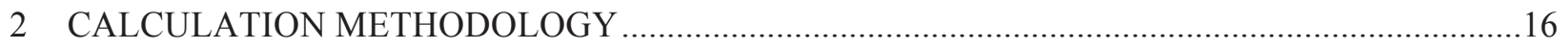

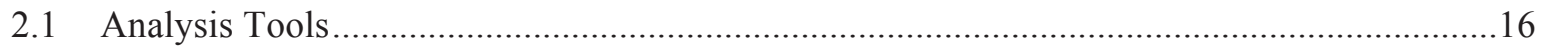

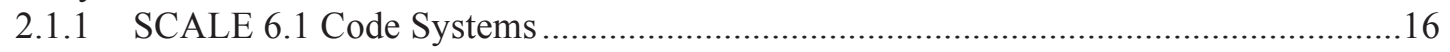

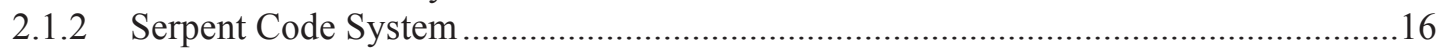

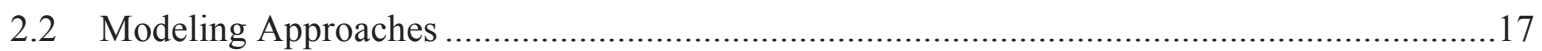

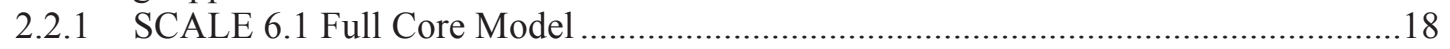

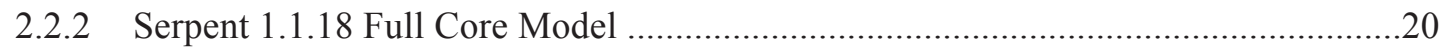

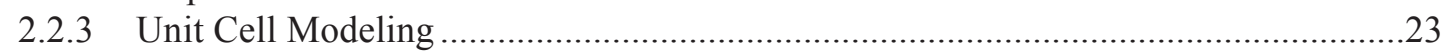

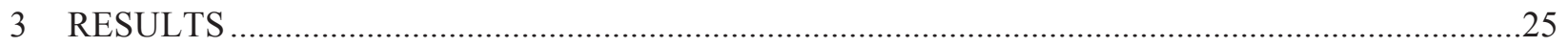

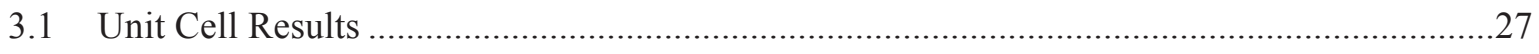

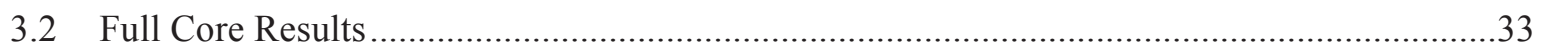

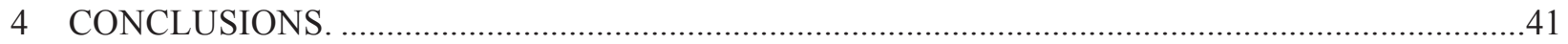

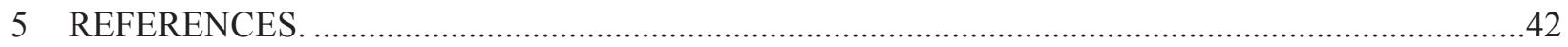

Appendix 1: Detailed isotopic masses (grams per initial ton HM) in fresh and discharged fuel for the Serpent Models.

\section{FIGURES}

Figure 1-1: MHTGR Reactor Plan view

Figure 1-2: Fuel Element Designs (units in inches): (a) Standard Fuel Element, (b) Reserve

Shutdown Fuel Element.

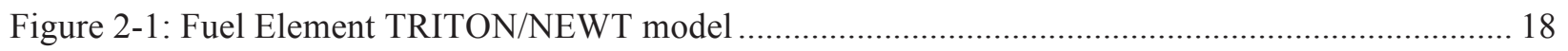

Figure 2-2: Reserve Shutdown Control (RSC) Fuel Element TRITON/NEWT model .......................... 19

Figure 2-3: $1 / 4^{\text {th }}$ symmetrical full core TRITON/NEWT model. ........................................................... 20

Figure 2-4: Fuel compact cutaway in xy coordinates .......................................................................... 21

Figure 2-5: A cutaway of full core Serpent model in xy coordinates.................................................... 22

Figure 2-6: A cutaway of Serpent model in xy coordinates. .............................................................. 23

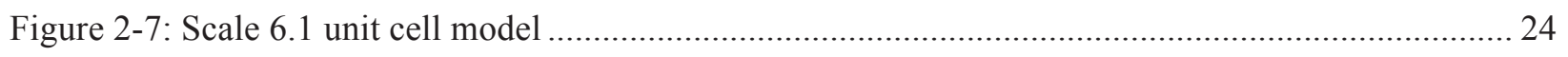

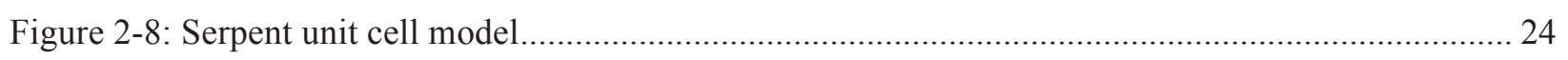

Figure 3-1: Ratio of capture in both U238 and Th232 to fission in both U233 and U235 ratio ................ 25

Figure 3-2: Capture in Th232 to fission in U233 ratio .......................................................................... 26 


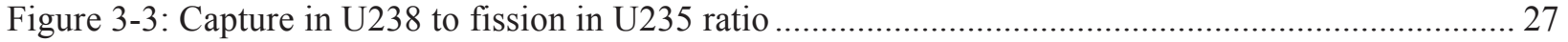

Figure 3-4: k-infinity trends during depletion for the unit cell models ................................................. 28

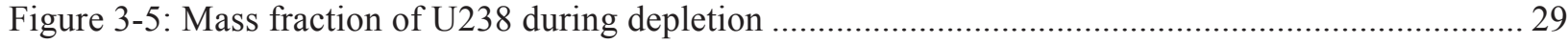

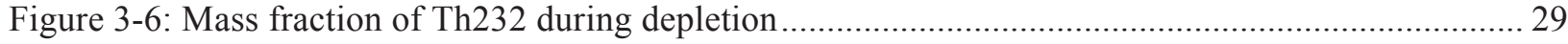

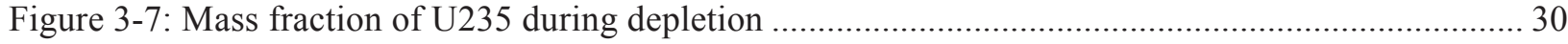

Figure 3-8: Mass fraction of U233 during depletion ....................................................................... 30

Figure 3-9: Mass fraction of Pu239 during depletion ........................................................................ 31

Figure $3-10: \mathrm{k}_{\infty}$ for different models during depletion ....................................................................... 32

Figure 3-11: k-effective during depletion for the full core model ......................................................... 34

Figure 3-12: U238 and Th232 mass fractions during depletion ........................................................ 35

Figure 3-13: U233 and Pu239 mass fractions during depletion........................................................... 35

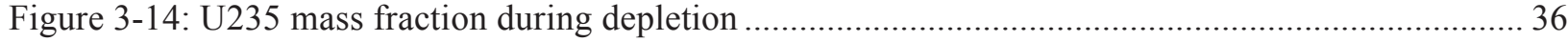

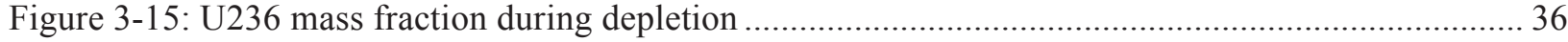

Figure 3-16: Np237 mass fraction during depletion ............................................................................. 37

Figure 3-17: Pu240 mass fraction during depletion................................................................................ 37

Figure 3-18: Pu241 mass fraction during depletion.............................................................................. 38

Figure 3-19: Pu242 mass fraction during depletion .............................................................................. 38

Figure 3-20: Am242m mass fraction during depletion ............................................................................. 39

TABLES

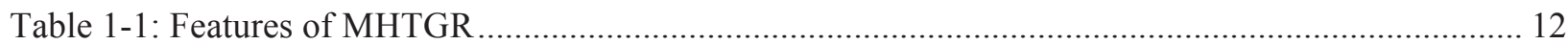

Table 1-2: Characteristics of the TRISO particles and the fuel compacts ............................................. 14

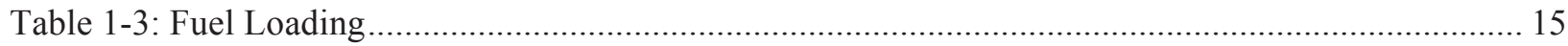

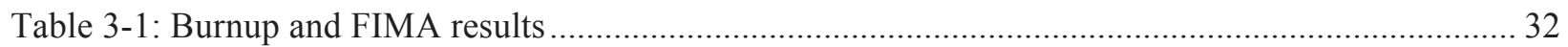

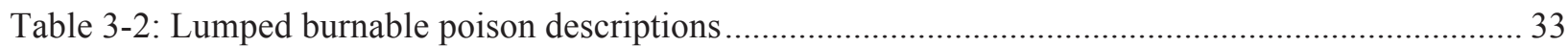

Table 3-3: Mass fractions of actinides at discharge for Serpent models .................................................. 39 
Depletion Analysis of Modular High Temperature Gas-cooled Reactor Loaded with LEU/Thorium Fuel

February 2013 


\section{ACRONYMS}

$\begin{array}{ll}\text { BOL } & \text { Beginning of Life } \\ \text { CRAM } & \text { Chebyshev Rational Approximation Method } \\ \text { DOE } & \text { Department of Energy } \\ \text { EFPD } & \text { Equivalent Full Power Days } \\ \text { EOL } & \text { End of Life } \\ \text { FIMA } & \text { Fissions per Initial Metal Atom } \\ \text { GA } & \text { General Atomics } \\ \text { GWd/tHM } & \text { Gigawatt-days per tonne of initial heavy metal } \\ \text { HTGR } & \text { High Temperature Gas-cooled Reactor } \\ \text { INL } & \text { Idaho National Laboratory } \\ \text { LBP } & \text { Lumped Burnable Poison } \\ \text { LEU } & \text { Low-Enriched Uranium } \\ \text { MHTGR } & \text { Modular High Temperature Gas-cooled Reactor } \\ \text { PyC } & \text { Pyrolitic Carbon } \\ \text { RSC } & \text { Reserve Shutdown Control } \\ \text { TRISO } & \text { Tri-isotropic }\end{array}$


Depletion Analysis of Modular High Temperature Gas-cooled Reactor Loaded with LEU/Thorium Fuel

February 2013 


\section{DEPLETION ANALYSIS OF MODULAR HIGH TEMPERATURE GAS-COOLED REACTOR LOADED WITH LEU/THORIUM FUEL}

\section{INTRODUCTION}

Thorium based fuel has been considered as an option to uranium-based fuel, based on considerations of resource utilization (Thorium is more widely available when compared to Uranium). The fertile isotope of Thorium (Th-232) can be converted to fissile isotope U-233 by neutron capture during the operation of a suitable nuclear reactor such as High Temperature Gas-cooled Reactor (HTGR). However, the fertile Thorium needs a fissile supporter to start and maintain the conversion process such as U-235 or Pu-239.

This report presents the results of a study that analyzed the thorium utilization in a prismatic HTGR, namely Modular High Temperature Gas-Cooled Reactor (MHTGR) that was designed by General Atomics (GA). The collected for the modeling of this design come from Chapter 4 of MHTGR Preliminary Safety Information Document that GA sent to Department of Energy (DOE) on 1995 [1].

The general features of the MHTGR are given in Table 1-1. MHTGR produces $350 \mathrm{MW}_{\text {th }}$ power at a 5.9 $\mathrm{W} / \mathrm{cm}^{3}$ power density and fueled with $\mathrm{UCO}+\mathrm{ThO}_{2}$ particles placed in fuel compacts. The inlet temperature of the helium coolant is $259^{\circ} \mathrm{C}$ while it heats up in the reactor core and reaches to an outlet temperature of $687^{\circ} \mathrm{C}$.

Table 1-1: Features of MHTGR

\begin{tabular}{|l|r|}
\hline Fuel & $\mathrm{UCO}+\mathrm{ThO}_{2}$ particles \\
\hline Coating & Ceramic $(\mathrm{PyC} / \mathrm{SiC} / \mathrm{PyC})$ \\
\hline Moderator & Graphite \\
\hline Coolant & Helium \\
\hline Coolant Boundary & Steel Pressure Vessel \\
\hline Power per Module & $140 \mathrm{MW} / 350 \mathrm{MW}$ th \\
\hline Power Density $\left(\mathrm{W} / \mathrm{cm}^{3}\right)$ & 5.9 \\
\hline Fuel Temperature $(\mathrm{Max} / \mathrm{Ave})\left({ }^{\circ} \mathrm{C}\right)$ & $1060 / 677$ \\
\hline Coolant Temperature $(\mathrm{In} / \mathrm{Out})\left({ }^{\circ} \mathrm{C}\right)$ & $259 / 687$ \\
\hline Coolant Pressure $(\mathrm{MPa})$ & 6.4 \\
\hline Steam Temperature $\left({ }^{\circ} \mathrm{C}\right)$ & 541 \\
\hline Steam Pressure $(\mathrm{MPa})$ & 17.2 \\
\hline
\end{tabular}

Figure 1-1 shows a cross section of the reactor core. The active core consists of hexagonal fuel elements, which are stacked in columns forming an annulus with columns of hexagonal graphite elements in the central and surrounding the active core. The central reflector allows a higher core power level while ensuring the peak fuel temperatures are limited in accident conditions.

The reactor is controlled by two independent means; the control rod system located in the reflector blocks adjacent to the active core (6 inner and 24 outer) and the reserve shutdown system located in the inner active core blocks (12). 


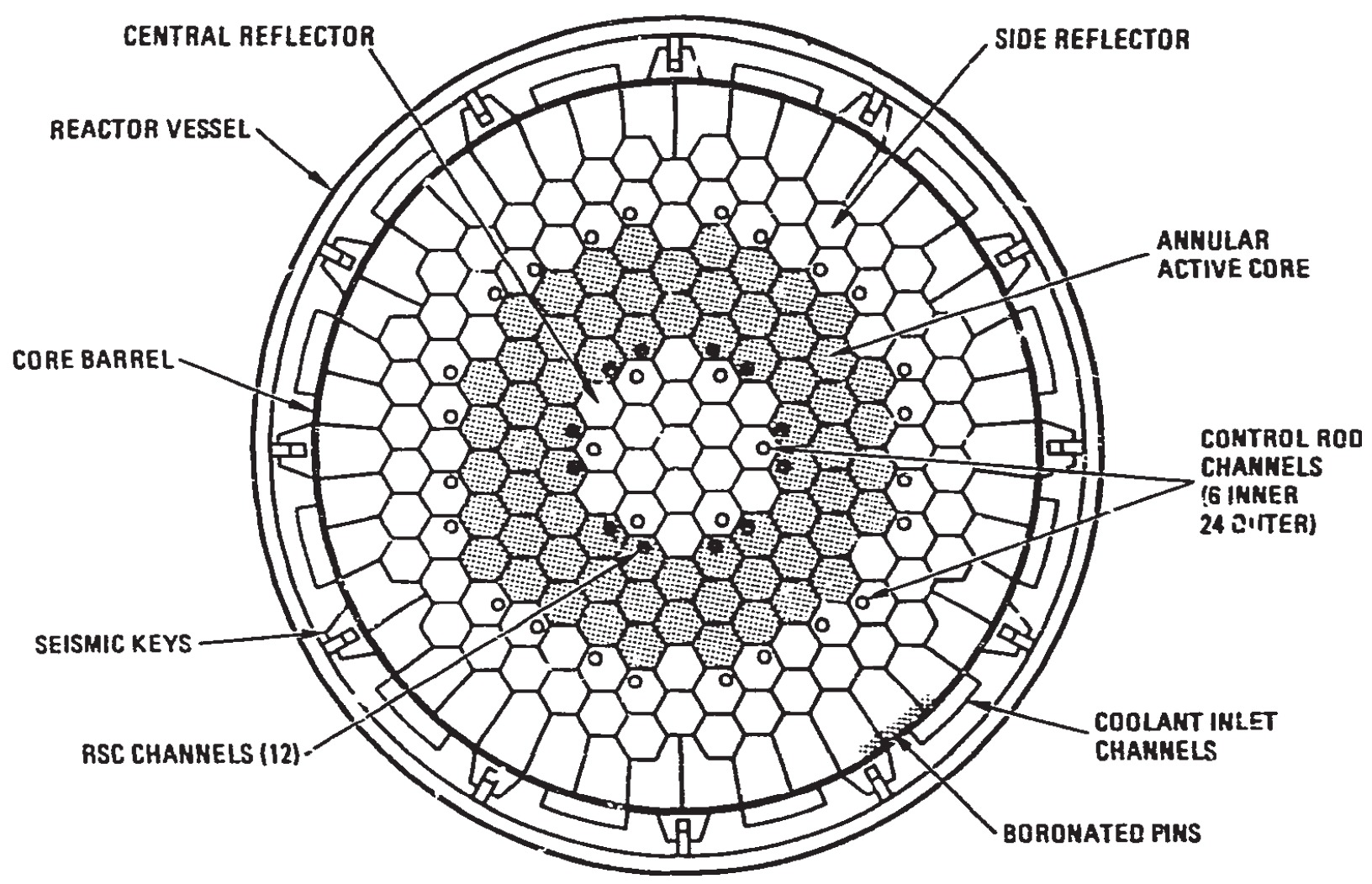

Figure 1-1: MHTGR Reactor Plan view

TRISO coated particles are used in MHTGR. The fuel kernels are coated with a porous graphite buffer to absorb gaseous fission products and provide a space for fission expansion. Two external coatings of pyrolytic carbon and one external coating of silicon carbide are added to retain the fission products within the fuel particle and buffer region. Both $\mathrm{UCO}$ and $\mathrm{ThO}_{2}$ kernels are manufactured in same configuration. The TRISO particles bonded by carbonaceous matrix are packed in fuel compacts, which are then stacked in each of the fuel element fuel holes. There are two types of fuel element; the standard fuel elements, and the RSC fuel elements that have a channel for reserve shutdown control material. The dimensions and the characteristics of the fuel elements are illustrated in Figure 1-2. The standard fuel element contains a continuous pattern of fuel and coolant holes except in for a central handling hole surrounded by smaller coolant holes and corner holes in which the fuel is replaced with lumped burnable poison (LBP). The reserve shutdown fuel elements differ from standard fuel elements that they contains a channel for reserve shutdown material. The LBP consists of boron carbide $\left(\mathrm{B}_{4} \mathrm{C}\right)$ granules dispersed in graphite rods. The $\mathrm{B}_{4} \mathrm{C}$ granules are pyrocarbon (PyC) coated to limit oxidation and loss from the system. The dimensions and the amount of the burnable poisons are not given in [1], however, the amount is determined by the reactivity requirements of each load and the size and the concentration are specified according to the requirements for self-shielding of the absorber to control its burnout rate relative to the fissile fuel burnout rate. 

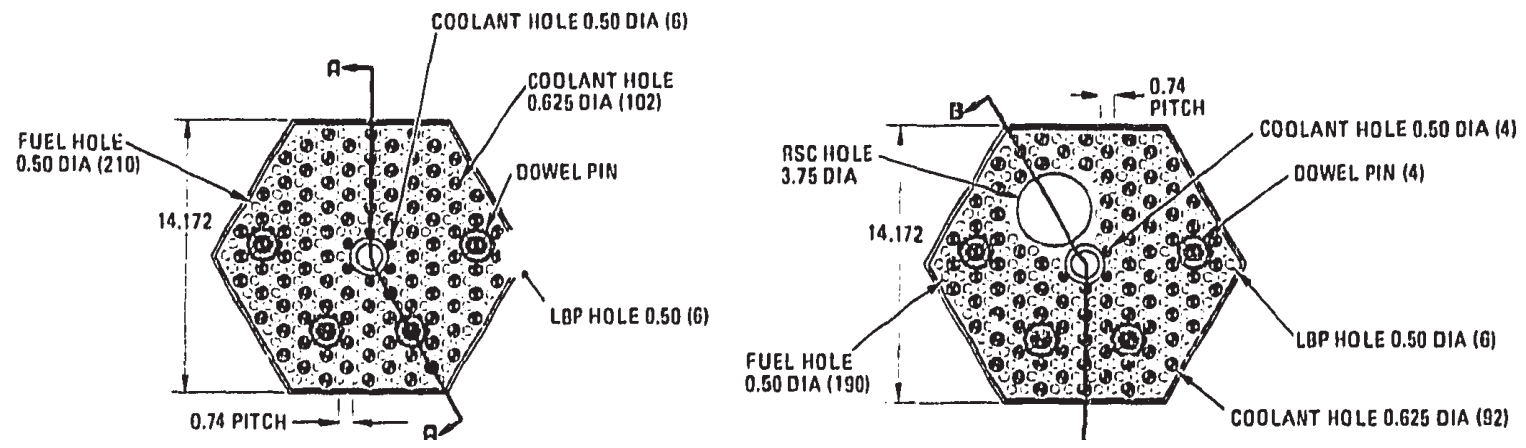

(a)

(b)

Figure 1-2: Fuel Element Designs (units in inches): (a) Standard Fuel Element, (b) Reserve Shutdown Fuel Element

The reference fuel cycle employs low-enriched uranium and thorium (LEU/Th). The fissile fuel is 19.9\% enriched UCO, having an oxygen-to-uranium ratio of 1.7 in the fresh fuel. The details of the TRISO particle and fuel compact design are given in Table 1-2.

Table 1-2: Characteristics of the TRISO particles and the fuel compacts

\begin{tabular}{|l|r|r|}
\hline \multicolumn{3}{|c|}{ TRISO Particle Design } \\
\hline Composition & $\mathrm{UC}_{0.3} \mathrm{U}_{1.7}$ & $\mathrm{ThO}_{2}$ \\
\hline Fuel kernel diameter $(\mu \mathrm{m})$ & 350 & 500 \\
\hline Buffer layer thickness $(\mu \mathrm{m})$ & 100 & 65 \\
\hline Inner PyC layer thickness $(\mu \mathrm{m})$ & 50 & 50 \\
\hline SiC layer thickness $(\mu \mathrm{m})$ & 35 & 35 \\
\hline Outer PyC layer thickness $(\mu \mathrm{m})$ & 40 & 40 \\
\hline Fuel kernel density $\left(\mathrm{g} / \mathrm{cm}^{3}\right)$ & 10.70 & 9.80 \\
\hline Buffer layer density $\left(\mathrm{g} / \mathrm{cm}^{3}\right)$ & 1.00 & 1.00 \\
\hline Inner PyC layer density $\left(\mathrm{g} / \mathrm{cm}^{3}\right)$ & 1.90 & 1.90 \\
\hline SiC layer density $\left(\mathrm{g} / \mathrm{cm}^{3}\right)$ & 3.20 & 3.20 \\
\hline Outer PyC layer density $\left(\mathrm{g} / \mathrm{cm}^{3}\right)$ & 1.87 & 1.87 \\
\hline \multicolumn{2}{|c|}{ Fuel Compact Design $^{3}$} & \\
\hline Diameter $(\mathrm{mm})$ & \multicolumn{2}{|c|}{$0.8-1.2$} \\
\hline Length $(\mathrm{mm})$ & \\
\hline Matrix density $\left(\mathrm{g} / \mathrm{cm}^{3}\right)$ & & 49.3 \\
\hline
\end{tabular}

The core incorporates a graded LEU/Th fuel cycle with an initial cycle length of 1.9 years. Equilibrium burnup cycles are designed to be 3.3 equivalent full power years (EFPD) and one half of the active core being replaced each 1.65 years. Before reaching the equilibrium cycle, there are three transition reload cycles. Table 1-3 gives the fuel loading for the first seven cycles. This fuel cycle is based on a 66-column annular core, which operates at $350 \mathrm{MW}_{\text {th }}$, corresponding to a power density of $5.9 \mathrm{MW} / \mathrm{cm}^{3}$. 
Depletion Analysis of Modular High Temperature Gas-cooled Reactor Loaded with LEU/Thorium Fuel

February 2012

Table 1-3: Fuel Loading

\begin{tabular}{|l|r|r|r|r|r|r|}
\hline \multirow{2}{*}{ Cycle } & \multirow{2}{*}{ EFPD } & \multirow{2}{*}{ C/Th } & \multirow{2}{*}{ C/U } & \multirow{2}{*}{ C/HM } & \multicolumn{2}{c|}{ Fuel Loading (kg) } \\
\cline { 6 - 7 } & & & & \multicolumn{1}{c|}{ Uranium } & \multicolumn{1}{c|}{ Thorium } \\
\hline 1 & 555 & 600 & 834 & 349 & 1726.3 & 2346.3 \\
\hline 3 & 380 & 1000 & 784 & 440 & 922.8 & 707.1 \\
\hline 4 & 490 & 1000 & 722 & 419 & 1001.1 & 706.1 \\
\hline 5 & 450 & 1000 & 730 & 422 & 990.7 & 706.2 \\
\hline 6 & 482 & 1000 & 701 & 412 & 1030.6 & 705.7 \\
\hline 7 & 482 & 1000 & 689 & 408 & 1048.2 & 705.4 \\
\hline
\end{tabular}




\section{CALCULATION METHODOLOGY}

\subsection{Analysis Tools}

The main purpose of this study is to provide the mass balances to the fuel cycle campaign. Therefore, SCALE 6.1/TRITON/ORIGEN code system is the primary code to be used in the system due to ORIGEN's extensive isotope library. The Serpent Monte-Carlo Depletion code is the secondary code used in the analysis to check the results for any errors in the models. A small overview on the main capabilities and models used is reported in the following paragraphs.

\subsubsection{SCALE 6.1 Code Systems}

The reactor physics calculations have been performed by the lattice physics capabilities of the SCALE 6.1 code systems. The calculation flow consists of the use of several modules mutually coupled. The discrete-ordinates code NEWT (New ESC-based Weighting Transport code) coupled to the depletion code ORIGEN [2] via the TRITON control module [3]. Using the discrete-ordinates approximation to the transport equation on an arbitrary grid, together with a 238-group neutron cross-section library based on ENDF/B-VII, NEWT provides a robust and rigorous deterministic solution for non-orthogonal configurations. The differencing scheme employed by NEWT, the Extended Step Characteristic Approximation, allows a computational two-dimensional mesh based on arbitrary polygons. Such a mesh can be used to closely approximate curved or irregular surfaces to provide the capability to model problems that were formerly difficult or impractical to model directly with discrete-ordinates methods.

The TRITON control module performs the task of coordination of data transfer between various physics codes available within SCALE 6.1 and of invoking those codes in the proper sequence for a desired type of calculation. The high-fidelity nature of the NEWT solution in estimating angular flux distributions combined with the rigor of the ORIGEN depletion solver gives TRITON the capability to perform precise burnup-dependent physics calculations with few implicit approximations, and limited primarily by the accuracy of nuclide cross-sectional data. Such rigor may be necessary to capture the unique attributes of Thorium fuel behavior as well as that of advanced, highly heterogeneous fuel assembly designs being deployed in current-generation reactors. Cross-sectional self-shielding is carried out by BONAMI for unresolved-range resonance data; the resolved resonance processor module CENTRM performs a 1-D discrete-ordinates code that uses point-wise cross-section data to produce a set of continuous-energy fluxes at discrete spatial intervals for each unit cell. Following a CENTRM calculation, the code PMC uses the resulting flux to collapse the point-wise continuous-energy cross sections into multi-group cross sections for each nuclide in each material in a unit (e.g., pin cell). The result is a multi-group library in which point cross-sectional data are weighted using the explicit point-wise spectrum representative of the nuclides present in a pin cell. Effects from overlapping resonances, fissile material in the fuel and surrounding moderator, anisotropic scattering, and inelastic level scattering are explicitly handled by this approach.

\subsubsection{Serpent Code System}

Serpent continuous-energy Monte Carlo reactor physics burnup calculation code [4] developed at the VTT Technical Research Centre of Finland. Serpent code reads continuous-energy interaction data from 
ACE format cross-section libraries. It contains libraries based on JEF2-2, JEFF-3.1, ENDF/b-VI.8 and ENDF/B-VII evaluated data files. Radioactive decay and fission yield data is needed for running the Serpent code in the burnup calculation mode. The decay constants and fission product distributions are read from standardized ENDF format data files. The format is directly accessible and the data requires no preprocessing. JEF-2.2, JEFF-3.1, ENDF/B-VI.8 and ENDF/B-VII data libraries are available within the code package.

The Serpent code uses a universe-based geometry model for describing complicated structures. This means that the geometry is divided into separate levels, which are all constructed independently and nested one inside the other. This approach allows the complexity of the geometry to be divided into smaller parts, which are much easier to handle. It also enables the use of regular geometry structures, such as square and hexagonal lattices, commonly encountered in reactor applications.

The fuels in high-temperature gas-cooled reactors (HTGR) consist of microscopic TRISO particles dispersed in a graphite matrix. The multi-layer particles can be simply defined with a regular lattice in regular arrangements. However, the regular arrangement fails to account for the random distribution of the particles and often leads to a distorted fuel-to-moderator ratio due to cell cut-off at the outer boundary. For this reason the Serpent code has two geometry models specifically designed for HTGR fuels.

The implicit particle fuel model works by sampling new particles on the neutron flight path during the tracking process. It should be noted that the model is not exact and there are statistically significant differences compared to the explicit model described below.

A better choice for modeling HTGR geometries is the explicit particle fuel model, which reads the positions of the particles from a separate file. The same model can be used for setting up reactor-scale pebble-bed geometries. The particle / pebble distribution is handled explicitly, so there are no approximations done in the modeling.

\subsection{Modeling Approaches}

The large neutron mean free paths in the HTGRs lead to difficulty capturing an appropriate spectrum in small-domain lattice calculations [5]. A method where the pitch of a single cell unit was modified to achieve the same averaged ratio of capture in U-238 to fission in U-235 (c8/f5) as in a whole core model was developed in previous studies [6]. Pope used the fresh fuel c8/f5 ratio to adjust their unit cell model to achieve the unit cell that would most closely represent the whole-core average condition, however during depletion the $\mathrm{c} 8 / \mathrm{f} 5$ ratio would change due to the change of the inventory. In a Thorium based fuel loaded core, the average ratio of capture in Th-232 to fission U-233 (c2/f3) will play role in representing the whole core conditions during depletion. Thus, in this study a whole-core model for depletion was chosen to pursue.

The fuel loadings from the equilibrium fuel cycle (Table 1-3) were used in the fuel models. It is assumed that the equilibrium cycle begins with a whole core loaded with fresh fuel and produces power for 964 EFPD (MHTGR has a 2-batch fuel cycle and each of the loading length is 482 EFPD), i.e. no transition cycle is modeled, since the shuffling methodology is not known.

The amount and the dimensions of the lumped burnable poisons are determined with the reactivity and self-shielding requirements for the full core depletion calculations. For the unit cell calculations no burnable poison is used. 


\subsubsection{SCALE 6.1 Full Core Model}

TRITON/NEWT module of SCALE6.1 system is used to model the whole core in 2-dimensions. The fuel compacts and the burnable poisons are modeled with the DOUBLEHET feature of the code system. Fuel compacts have two different grain types, which are modeled explicitly. A separate material number is used for the graphite surrounding the burnable poisons to take into account the spectrum effects. Fuel compacts, burnable poisons and helium coolant flow channels are modeled explicitly in the fuel elements. Figure 2-1 and Figure 2-2 shows the TRITON/NEWT models of fuel element and RSC fuel elements, respectively, where with different materials represented by different colors. Figure 2-3 shows the $1 / 4^{\text {th }}$ symmetrical full core model with the mesh grid. Reflected boundary conditions are used in the model.

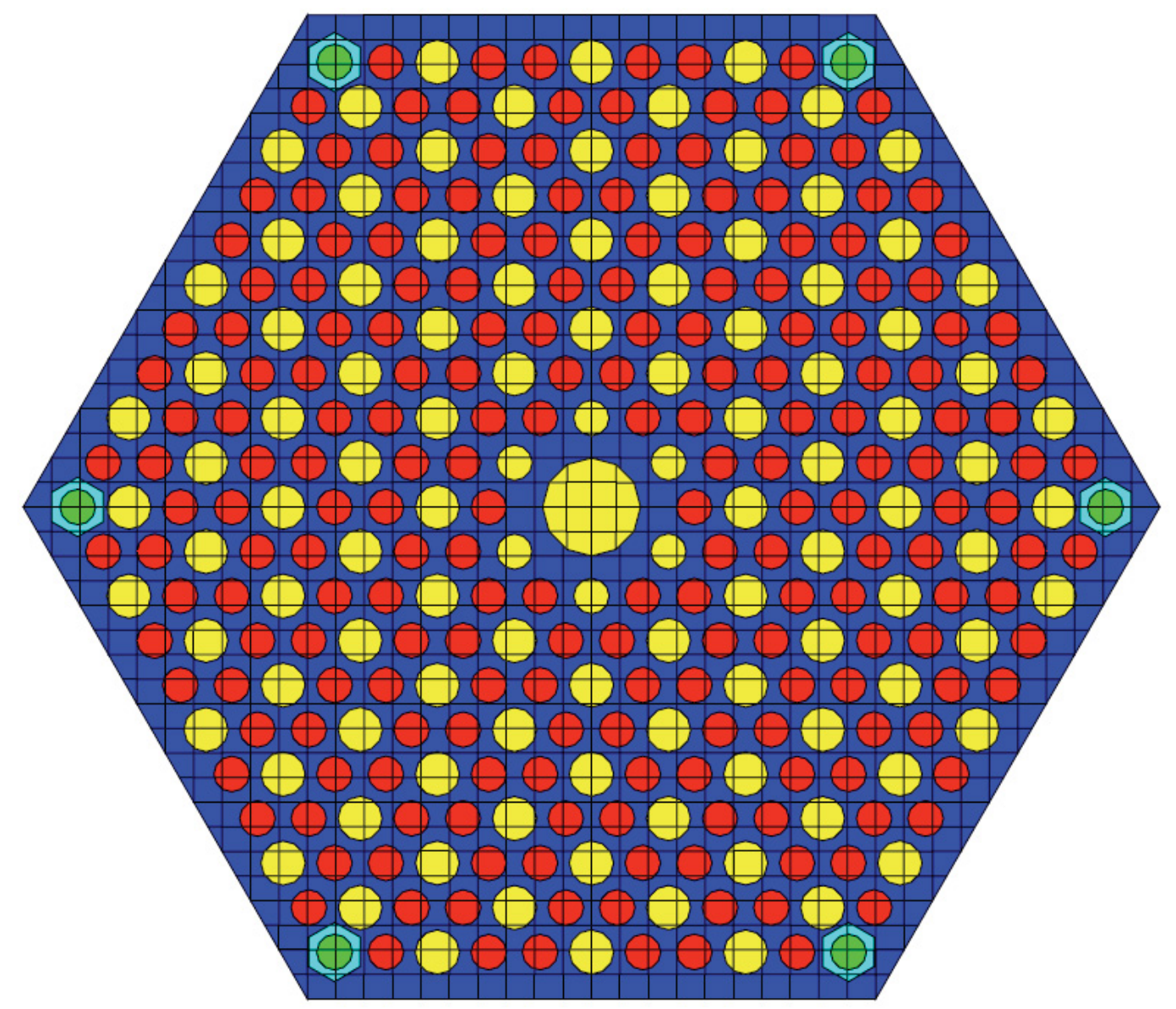

Figure 2-1: Fuel Element TRITON/NEWT model 


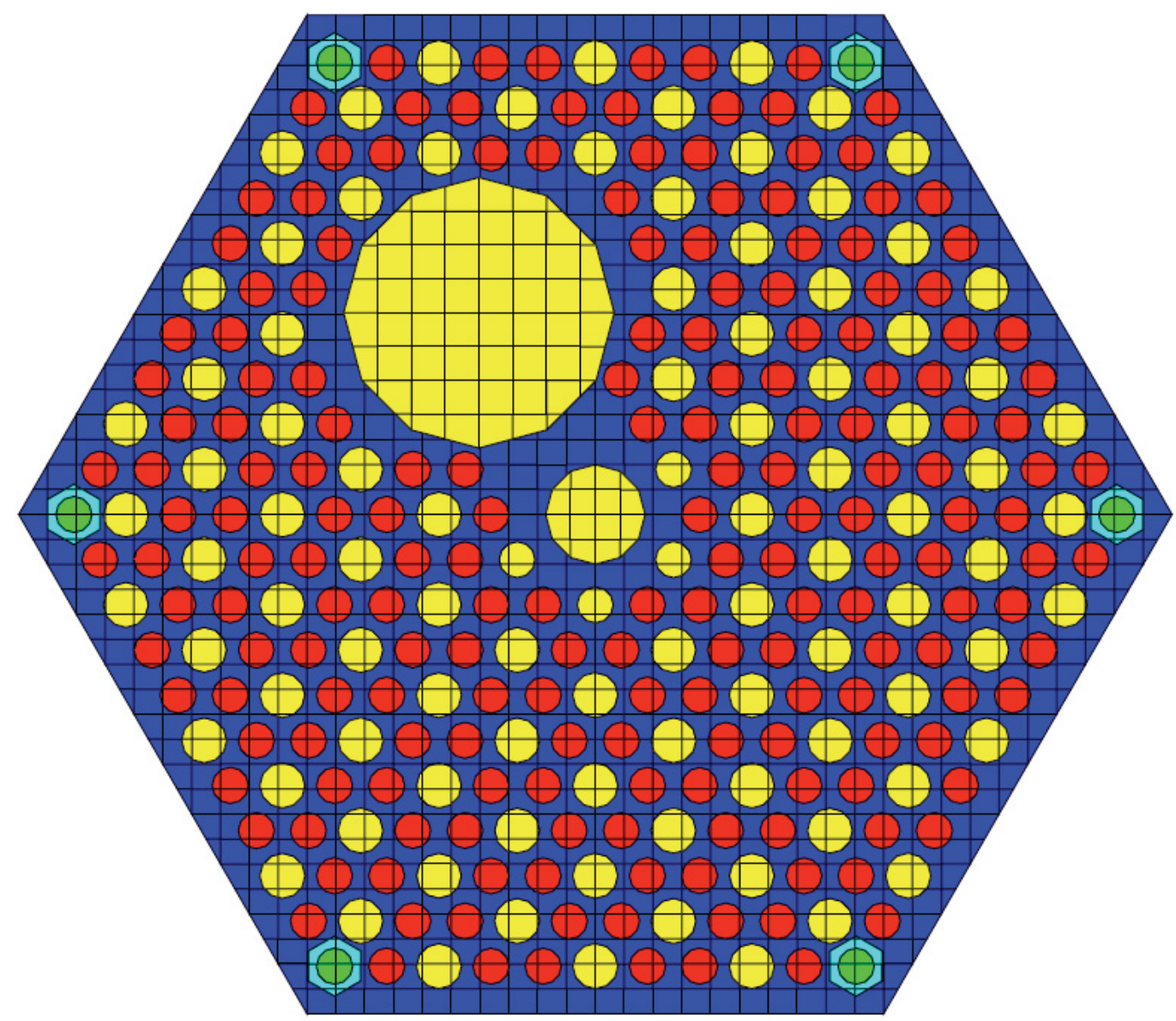

Figure 2-2: Reserve Shutdown Control (RSC) Fuel Element TRITON/NEWT model 


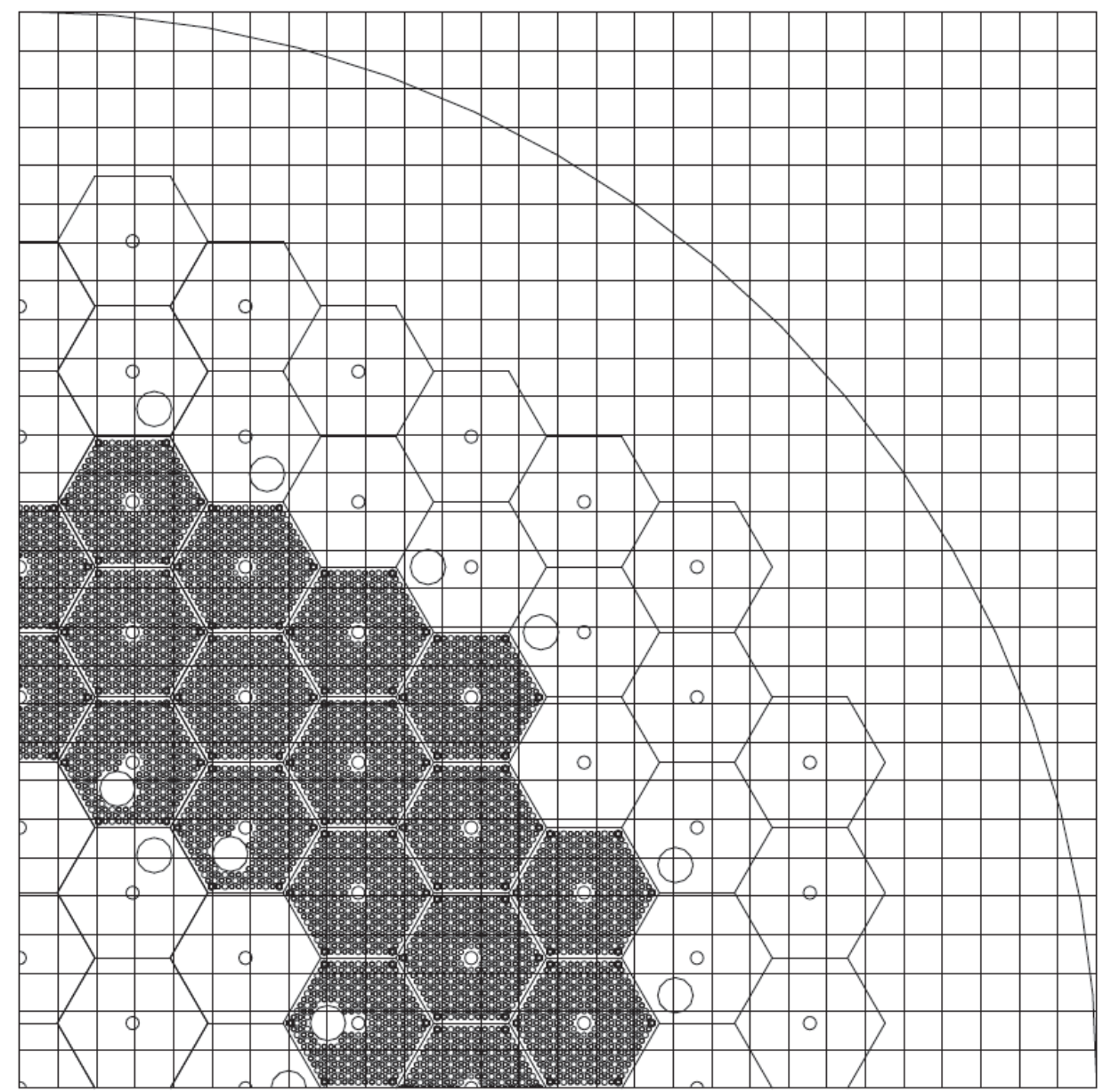

Figure 2-3: $1 / 4^{\text {th }}$ symmetrical full core TRITON/NEWT model.

The TRITON/NEWT calculations are carried out with using 238-group ENDF/B-VII library, using SN method.

The first efforts to simulate the whole core depletion with TRITON/NEWT model resulted with a domain that is so large that the calculations did not converge within a reasonable time frame. Therefore the same unit cell calculation methodology as in [6] was carried out with Scale6.1.

\subsubsection{Serpent 1.1.18 Full Core Model}

The two different types, $\mathrm{UCO}$ and $\mathrm{ThO}_{2}$, within the fuel compacts as well as the burnable poisons are modeled explicitly with using the random dispersion functionality of the Serpent code. A cutaway of a compact having two different types of grains, $\mathrm{UCO}$ and $\mathrm{ThO}_{2}$, with diameters of $0.0175 \mathrm{~cm}, 0.0250 \mathrm{~cm}$ and a packing fraction of $21.2 \%$ and $7.3 \%$, respectively, is shown in Figure 2-4. 


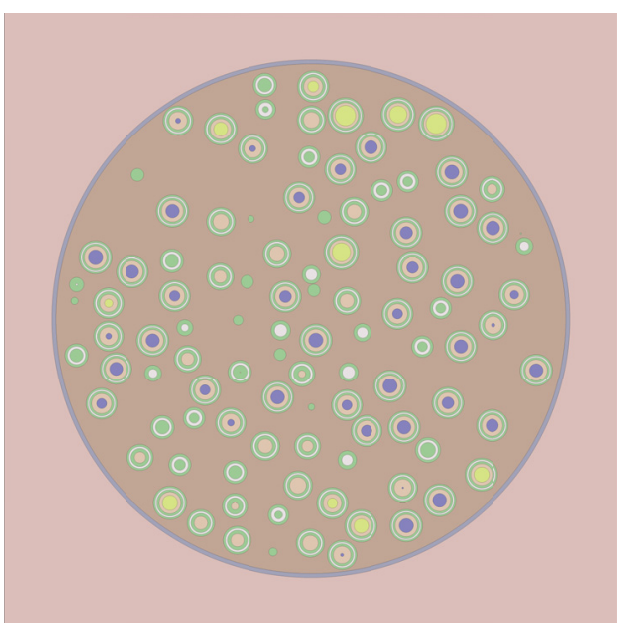

Figure 2-4: Fuel compact cutaway in $x y$ coordinates

Fuel compacts, burnable poisons and coolant channels are modeled explicitly in fuel elements and RSC fuel elements. The helium coolant gaps between the blocks are modeled explicitly. Figure 2-5 shows the entire domain of the Serpent full core model in xy coordinates, while Figure 2-6 shows a more close-up cutaway to the blocks where the fuel compacts with TRISO particles, burnable poisons, coolant and RSC channels and the coolant gaps between the blocks are seen explicitly.

Serpent is used as a stand-alone system for depletion calculations in which case the code uses an internal calculation routine for solving the set of Bateman equations describing the changes in the material compositions caused by neutron induced reactions and radioactive decay. The CRAM method option is used to solve the Bateman equations.

The cross section libraries based on ENDF/B-VII evaluated nuclear data are used for both neutronic and depletion calculations. 


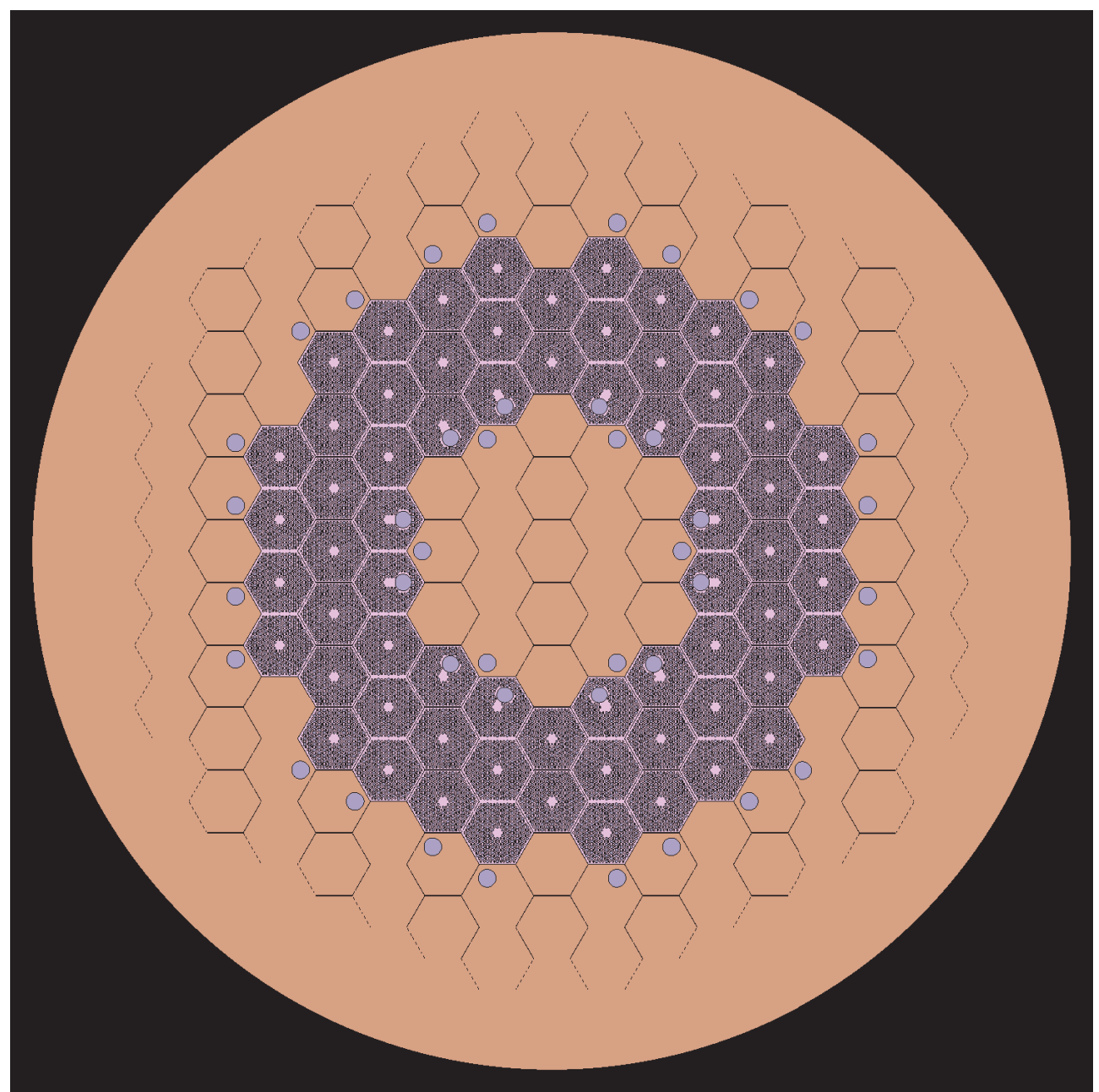

Figure 2-5: A cutaway of full core Serpent model in xy coordinates. 


\section{Depletion Analysis of Modular High Temperature Gas-cooled Reactor Loaded with LEU/Thorium Fuel}

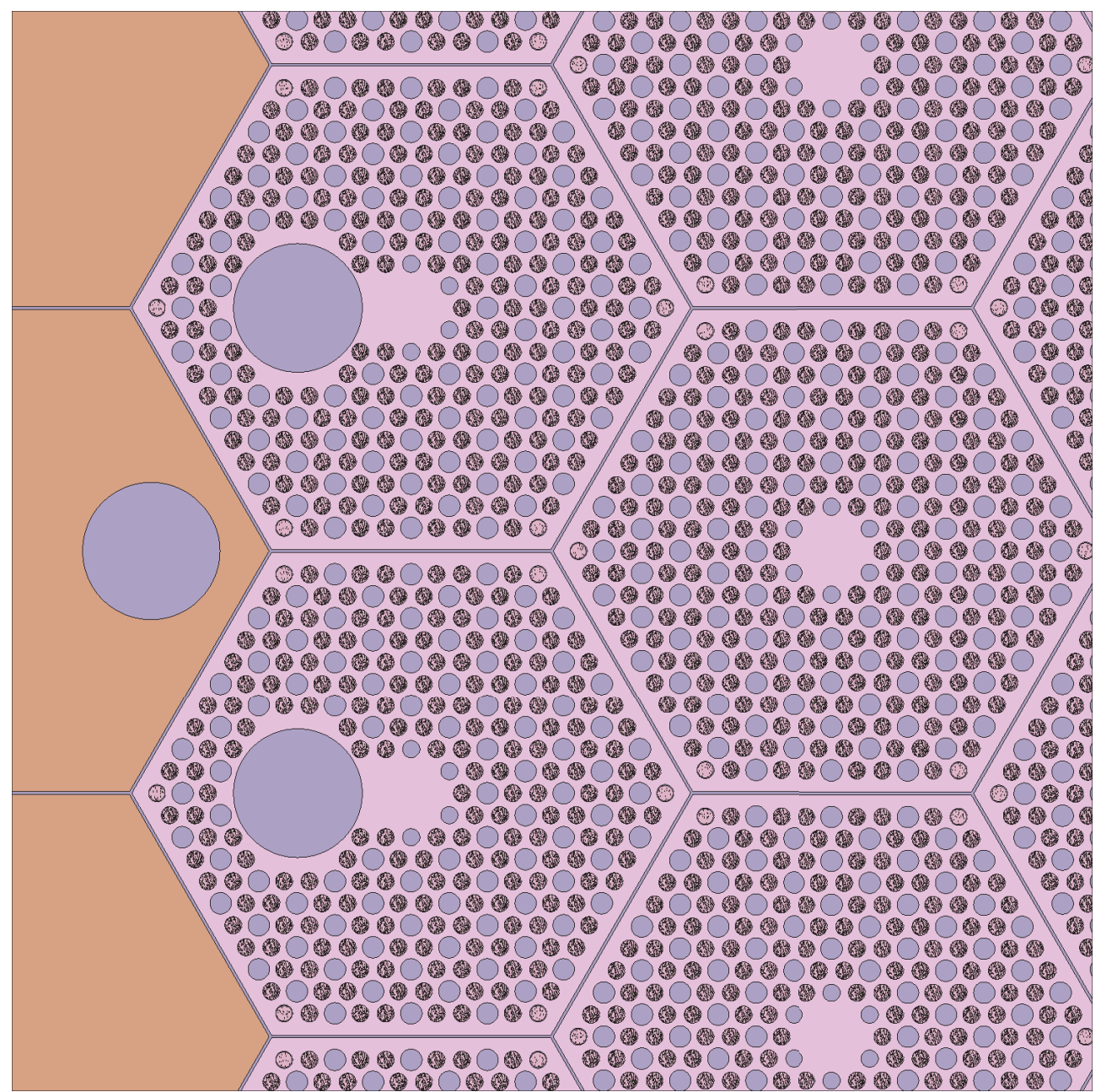

Figure 2-6: A cutaway of Serpent model in xy coordinates.

\subsubsection{Unit Cell Modeling}

A unit cell model developed by the methodology that is suggested by Pope. The full core model as described in section 2.2.2 is used to calculate the whole-core averaged ratio of capture in U-238 to fission in U-235 and the averaged ratio of capture in Th-232 to fission in U-233. The pitch dimension in the unit cell geometry is adjusted to get the same ratios as in the whole-core model. Figure 2-7 and Figure 2-8 show the unit cell models of Scale 6.1 and Serpent codes, respectively, that are used in the analysis. The fuel compact contains both $\mathrm{UCO}$ and $\mathrm{ThO}_{2}$ grain types. 
Figure 2-7: Scale 6.1 unit cell model
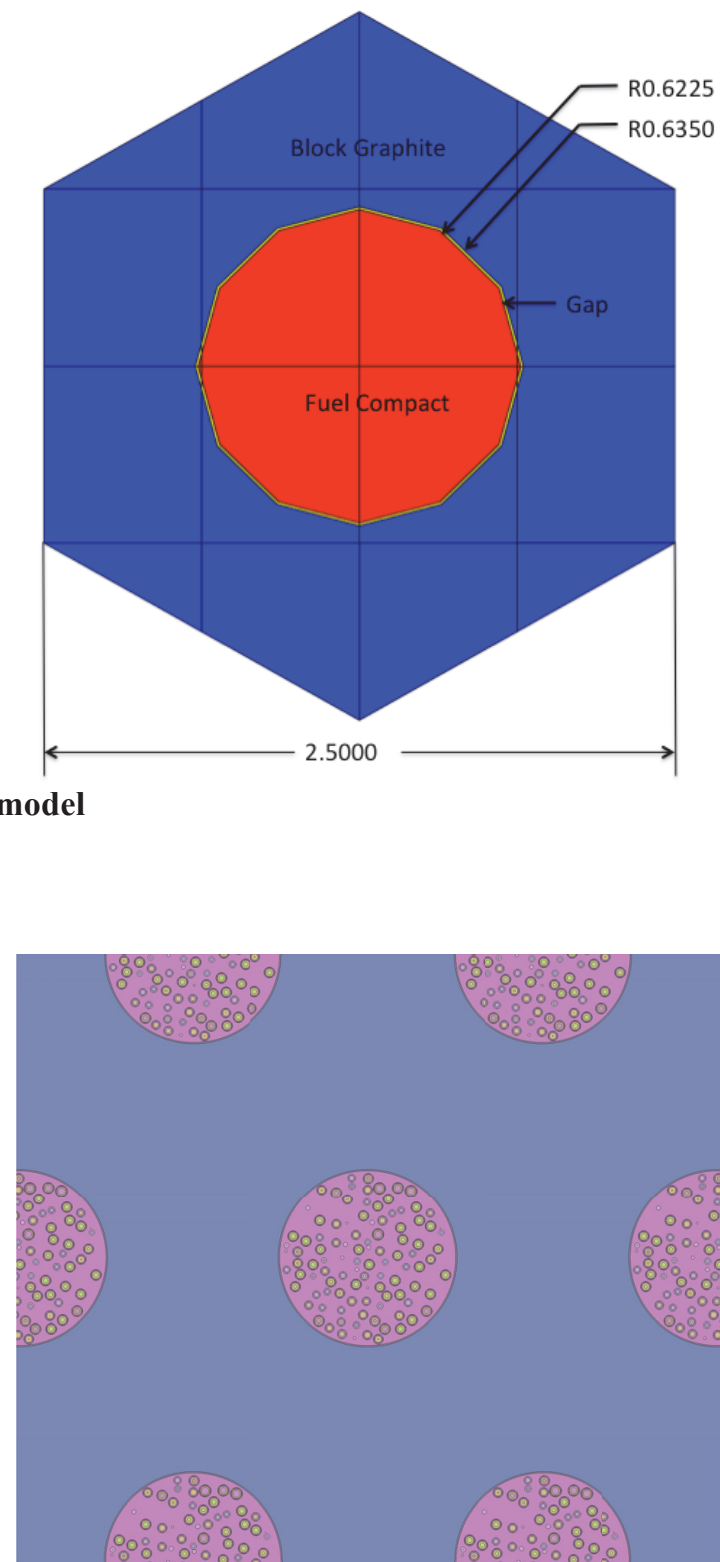

Figure 2-8: Serpent unit cell model 


\section{RESULTS}

The results of the analysis are presented in section. The main purpose of this report is to estimate the inventory of the MHTGR for the equilibrium cycle; therefore only the effective or infinite multiplication factor is presented in terms of the reactor physics characteristics of the MHTGR core.

The inventory of the core (from BOL to EOL), the production of U-233 from Th-232 and the transuranics are the main results discussed in this section.

The Serpent full core model is used to calculate the core-averaged ratio of capture (U238 and Th232) to fission (U235 and U233). This was selected as spectral index of interest because it is an indicator of the amount of heavy metal that is converted to fissile material as per the thermal power produced. Then a unit cell model, having the same fuel, developed and the pitch of the representative hexagonal unit cell was varied until the same capture to fission ratio is achieved. The effective pitch size was found to be around $2.5 \mathrm{~cm}$. Figure 3-1, Figure 3-2 and Figure 3-3 shows the ratio of capture in both U238 and Th232 to fission in both $\mathrm{U} 235$ and $\mathrm{U} 233$, the ratio of capture in U238 to fission in U235 and the ratio of capture in Th232 to fission in U233, respectively. The full core model results with and without lumped burnable poisons (LBP) are also shown. Due to the presence of the LBP the capture to fission ratio is higher at the beginning of the depletion. After the LBP is burned out the capture to fission ratio equalizes with the full core model without LBP.
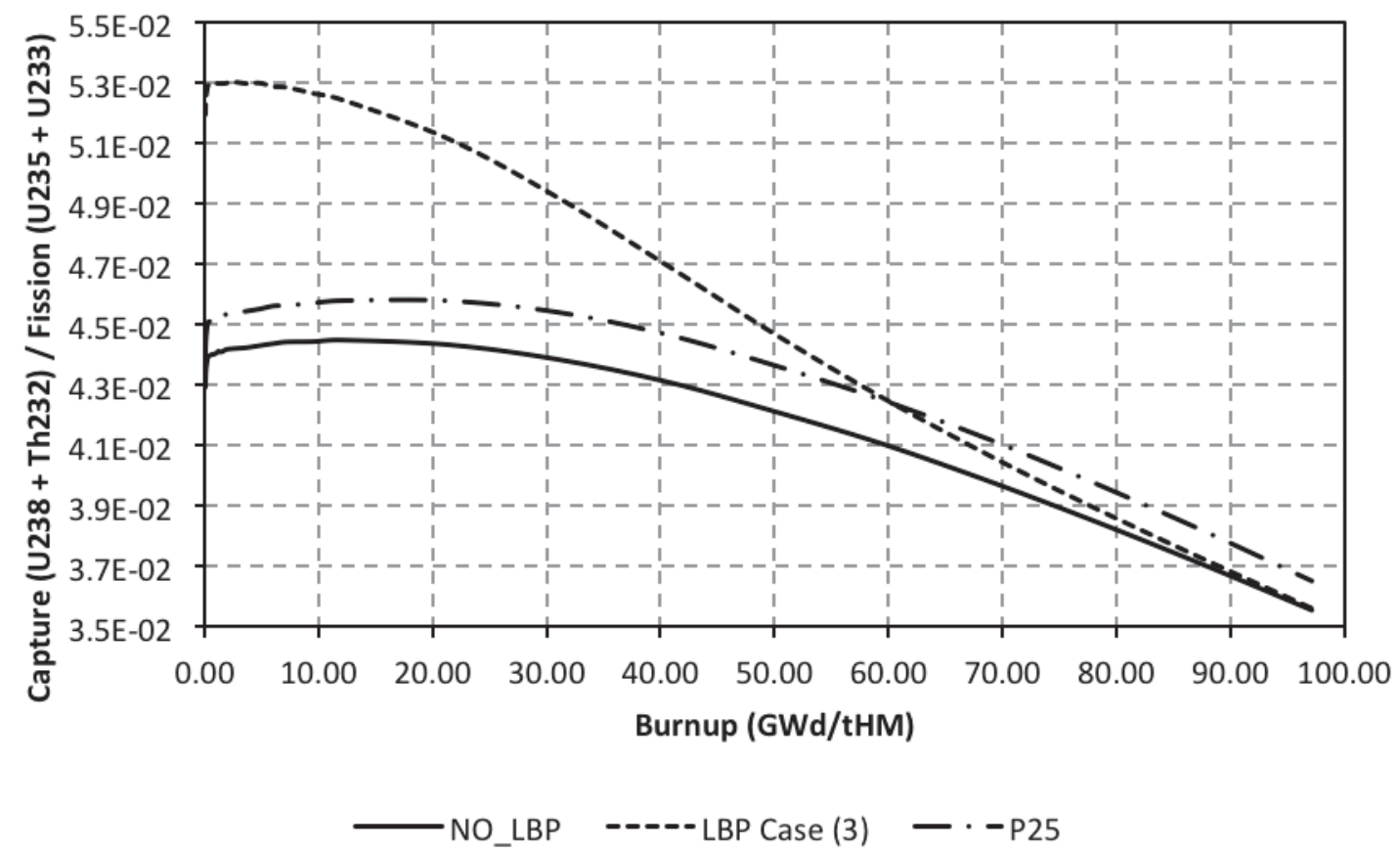

Figure 3-1: Ratio of capture in both U238 and Th232 to fission in both U233 and U235 ratio 


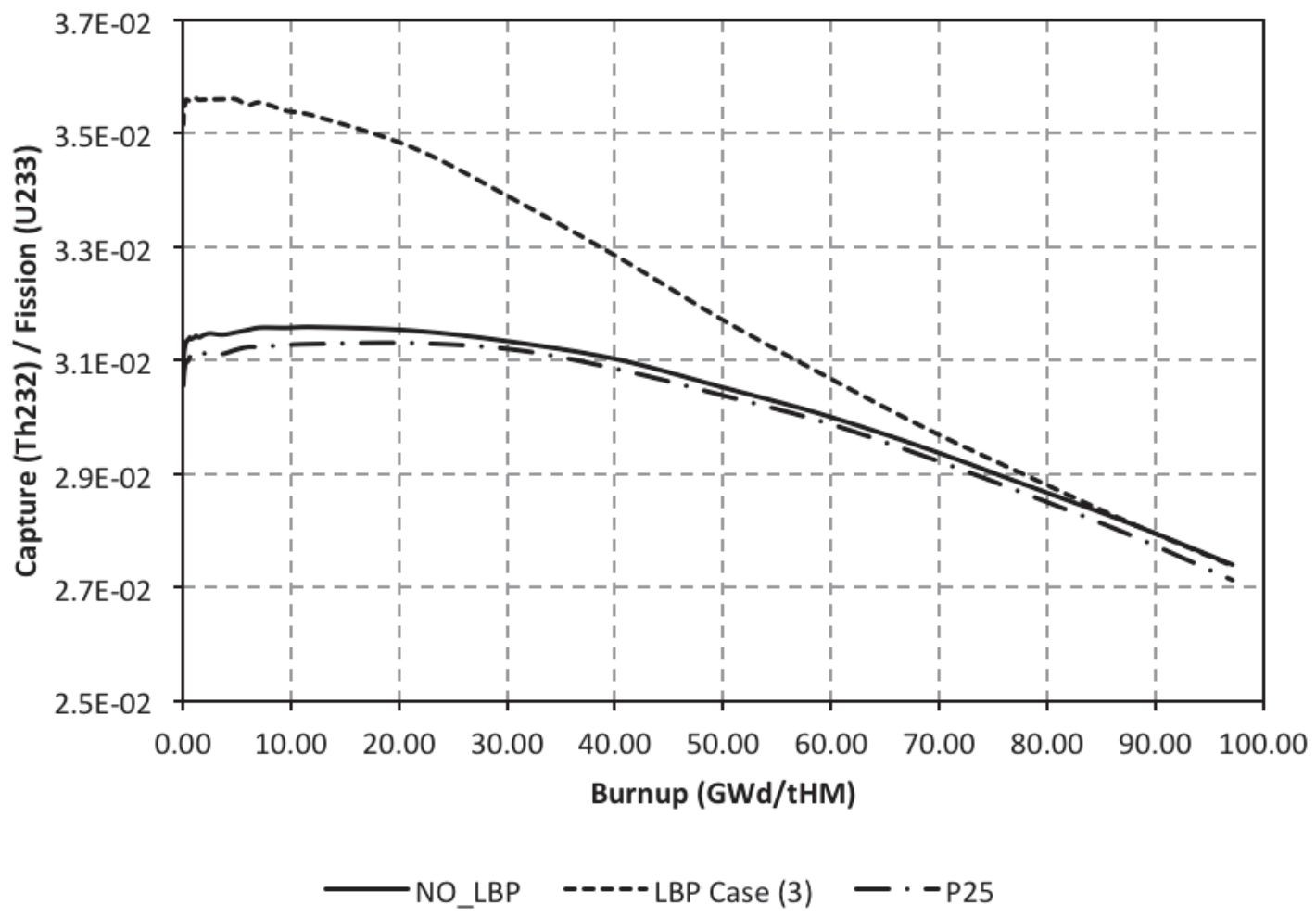

Figure 3-2: Capture in Th232 to fission in U233 ratio 

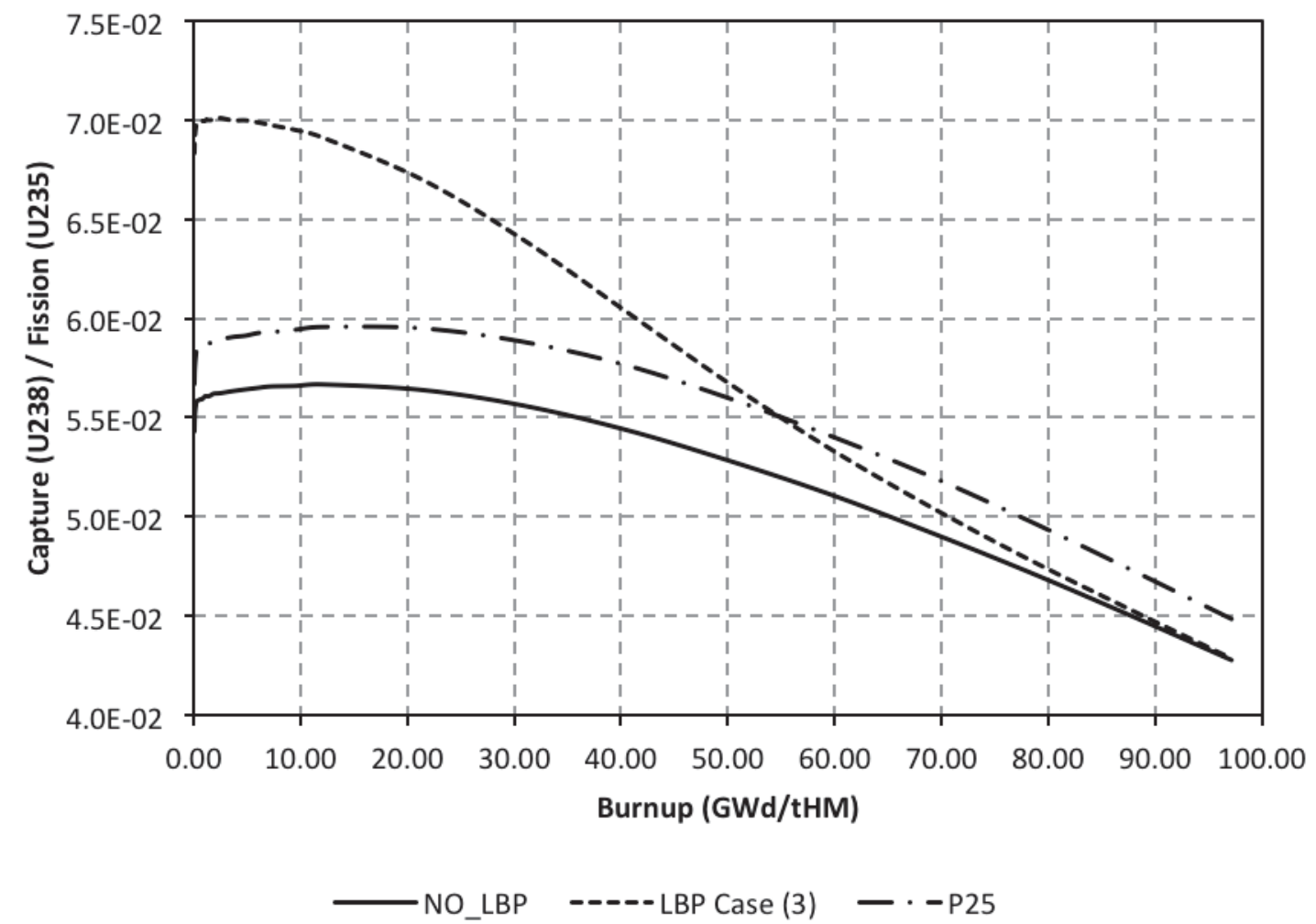

Figure 3-3: Capture in $\mathbf{U} 238$ to fission in $\mathrm{U} 235$ ratio

\subsection{Unit Cell Results}

For depletion calculations, it is important to add trace quantities $\left(1 \times 10^{-20}\right.$ atoms $\left./ \mathrm{b}-\mathrm{cm}\right)$ of certain nuclides to the inventories of depletion materials in order to accurately track the nuclides' impact on cross-section processing and transport calculations as a function of burnup. By default, TRITON automatically adds to all fuel materials trace quantities of a set of nuclides that have been determined to be important in the characterization of spent fuel. TRITON recognizes fuel materials as any material containing quantities of heavy metals $(Z>89)$ in the standard composition specification [7].

TRITON provides user control of the set of nuclides added to a fuel material through the parm $=($ addnux $=N)$ control parameter, where $\mathrm{N}$ is an integer value. For $\mathrm{N}=2$, the default setting for the TRITON depletion sequences, 94 nuclides are added. $\mathrm{N}=3$ and $\mathrm{N}=4$ add 230 and 388 nuclides, respectively; this much detail is generally not needed for depletion calculations unless one wishes to closely estimate $\mathbf{k}_{\text {eff }}$ near the end of life. At such high burnups, these nuclides have little effect on the system spectrum, but taken as a whole, they do contribute to the total system reactivity. The default in the SCALE 6.1 release remains parm= $(a d d n u x=2)$. For the calculation of MHTGR with LEU/Th fuel cycle, the control parameter parm $=(\boldsymbol{a d d n u x}=3)$ should be used, since $\mathbf{U} 233$, Pa233 is not added to the transport calculations with the default option.

The unit cell model $\mathrm{k}_{\infty}$ during depletion for Scale 6.1 with both addnux $=2$ and addnux $=3$ options and Serpent model are shown in Figure 3-4. The $\mathrm{k}_{\infty}$ for addnux $=2$ is much lower when compared to addnux=3 
and Serpent results, as expected. However, Scale6.1 with addnux=3 and Serpent results show significant differences as well, which needs more investigation.
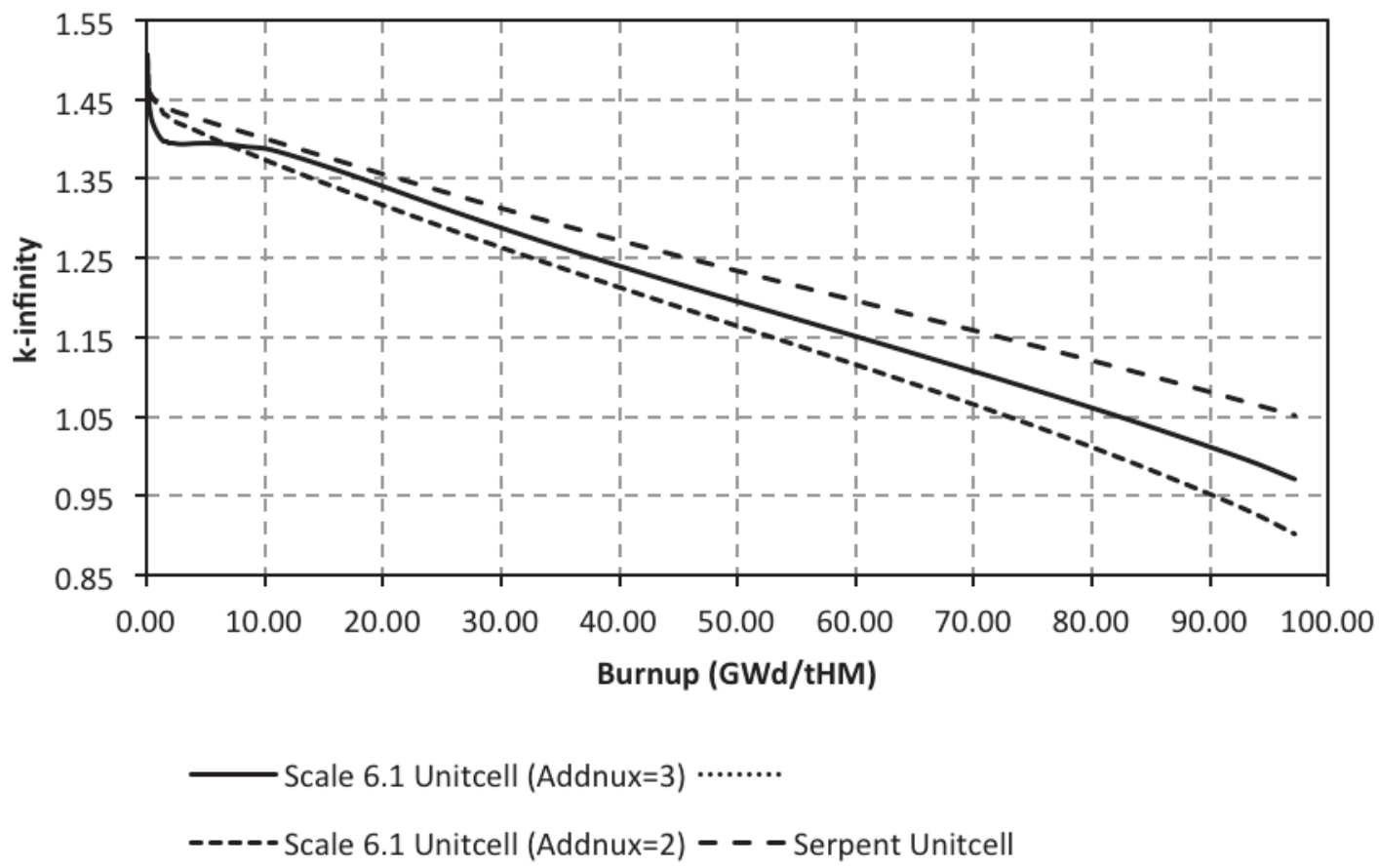

Figure 3-4: k-infinity trends during depletion for the unit cell models

The mass fractions of U238, Th232, U235, U233 and Pu239 during depletion are shown in Figure 3-5, Figure 3-6, Figure 3-7, Figure 3-8 and Figure 3-9, respectively. The mass fraction of U238 is lower in Scale 6.1 model during the depletion, while the Pu239 mass fraction is higher. This difference can be explained with the higher value of the ratio of capture in U238 to fission in U235 in Scale 6.1 model (Figure 3-3). However, the mass ratio of Th232 and U233 shows significant differences, which needs more investigation. 


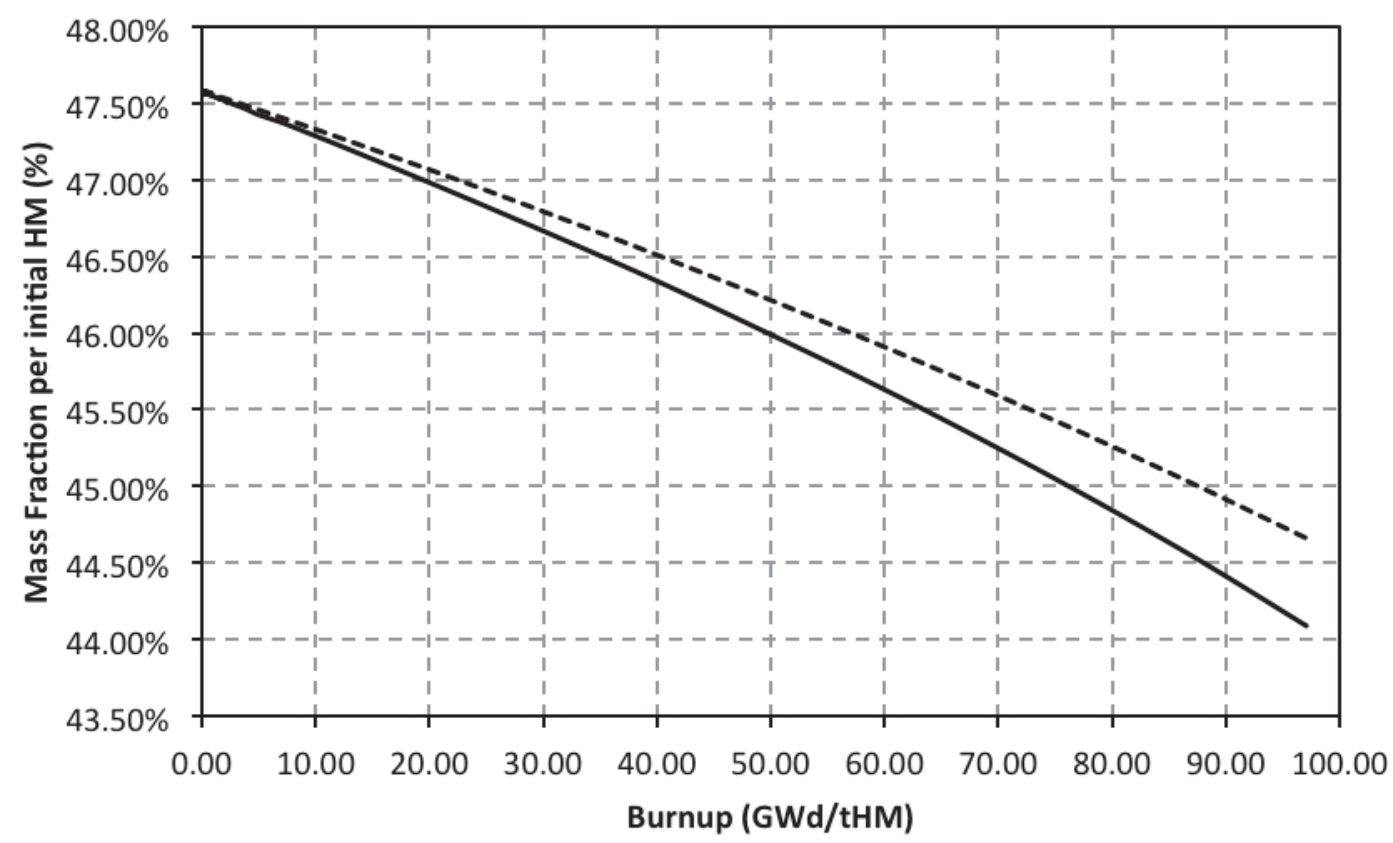

- Scale6.1 U238 ------Serpent U238

Figure 3-5: Mass fraction of U238 during depletion

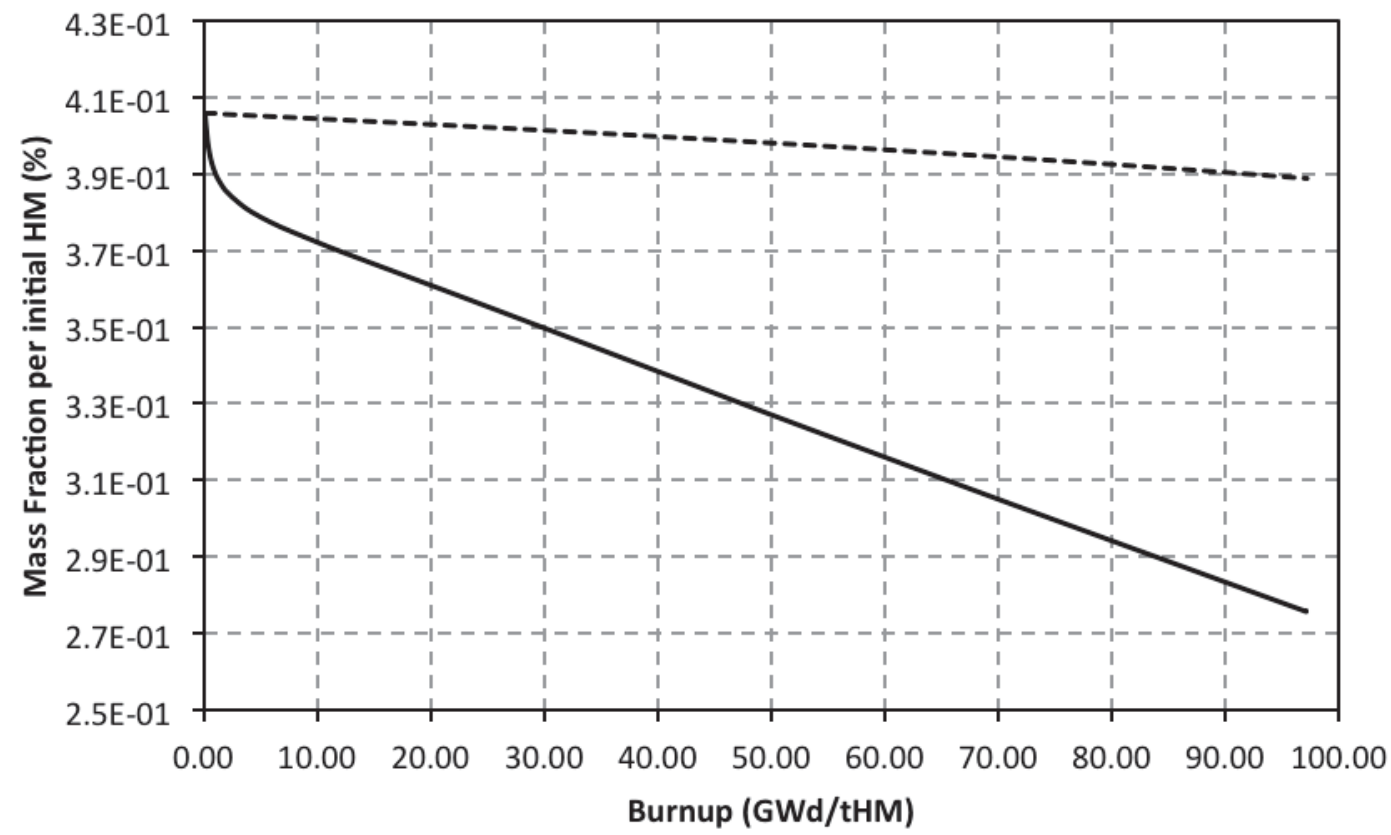

- Scale6.1 Th232 -----Serpent Th232

Figure 3-6: Mass fraction of Th232 during depletion 


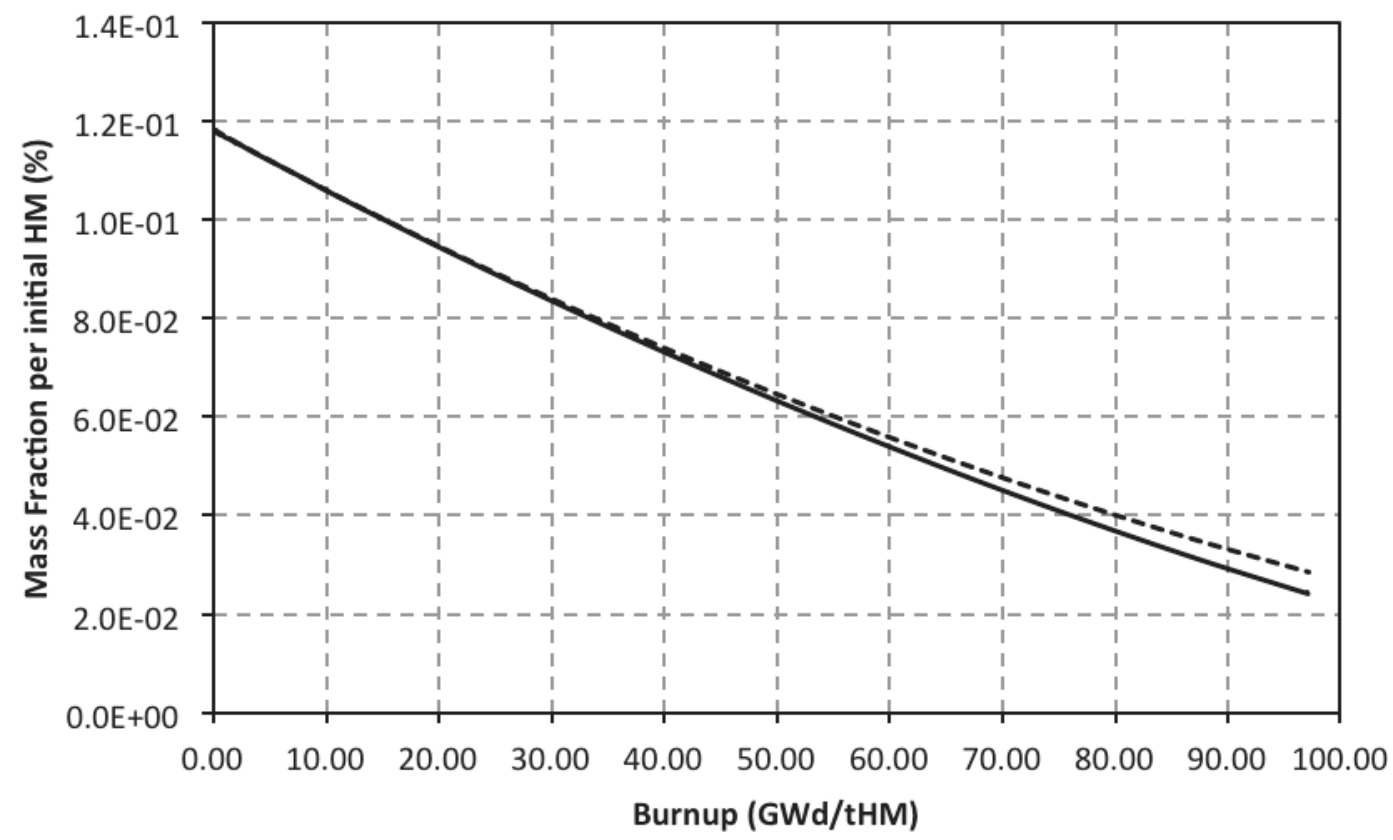

—-Scale6.1 U235 ------Serpent U235

Figure 3-7: Mass fraction of U235 during depletion

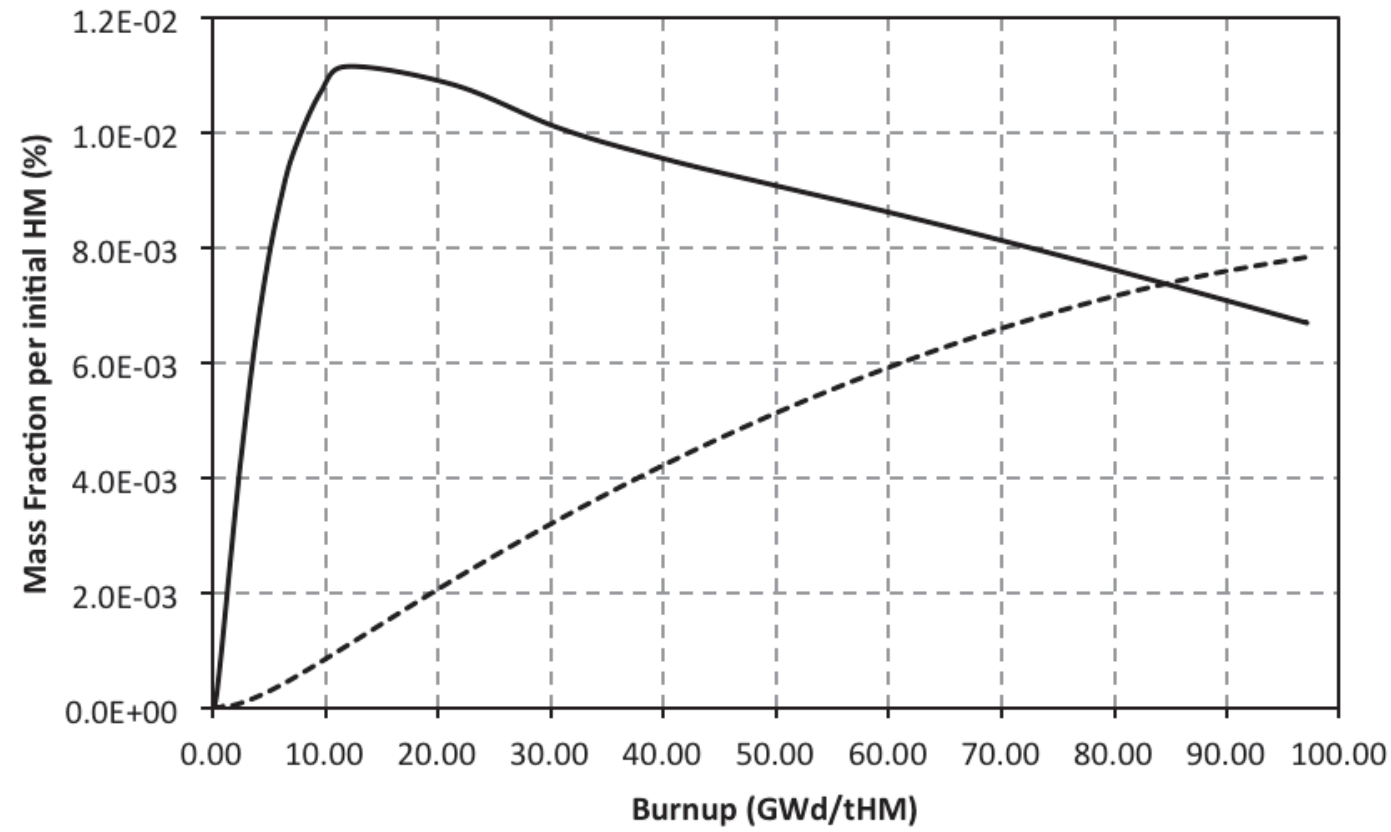

—Scale6.1 U233 ------Serpent U233

Figure 3-8: Mass fraction of U233 during depletion 


\section{Depletion Analysis of Modular High Temperature Gas-cooled Reactor Loaded with LEU/Thorium Fuel}

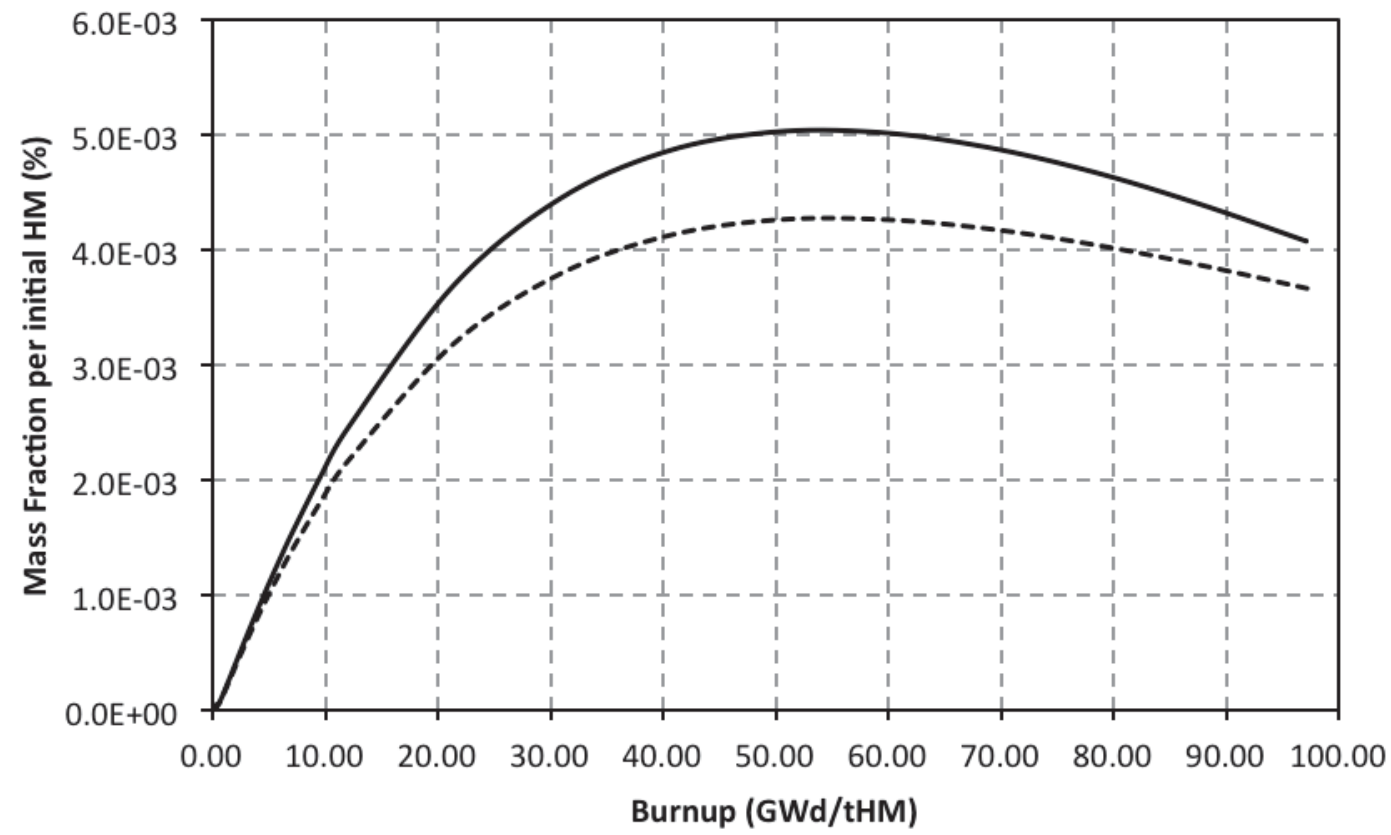

- Scale6.1 Pu239 -----Serpent Pu239

Figure 3-9: Mass fraction of Pu239 during depletion

For investigating the conversion/depletion of the heavy metals with burnup of the fuel fissions per initial metal atom (FIMA) is another useful figure of merit. If $10 \%$ of initial metal atoms have undergone fission, the burnup is $10 \%$ FIMA. FIMA can easily be calculated as the ratio of the mass of fission products to the initial mass of metal atoms $\left(\mathrm{FP}_{\mathrm{f}} / \mathrm{MA}_{\mathrm{i}}\right)$ or 1.0 minus the ratio of the mass of metal atoms to the initial mass of metal atoms $\left(1.0-\mathrm{MA}_{\mathrm{f}} / \mathrm{MA}_{\mathrm{i}}\right)$. As an approximation 10\% FIMA is just less than 100 $\mathrm{GWd} / \mathrm{tHM}$. So in our case the final burnup of $97 \mathrm{GWD} / \mathrm{tHM}$ is equivalent to $10 \%$ FIMA. Table 3-1 gives the calculated burnup and FIMA values for Scale 6.1 and Serpent. Discrepancies were observed in the Scale 6.1 model results; therefore several different models were developed to identify the reason of these discrepancies. To eliminate the effect of thorium cycle from the calculation both grain types were converted to UCO fuel, however the discrepancies between the burnup and the calculated FIMA were still observed. Then the second grain type was removed from the model, and a good agreement between the burnup and the calculated FIMA were observed. 
Table 3-1: Burnup and FIMA results

\begin{tabular}{|c|c|c|c|}
\hline Code & Model & $\begin{array}{l}\text { Burnup } \\
\text { (GWd/thM) }\end{array}$ & FIMA (\%) \\
\hline \multirow{5}{*}{ Scale 6.1} & \multirow{3}{*}{$\begin{array}{l}\text { Two type of grains }\left(\mathrm{UCO} \text { and } \mathrm{ThO}_{2}\right) \text { in fuel } \\
\text { compact }\end{array}$} & 1.21 & $0.23 \%$ \\
\hline & & 40.70 & $10.86 \%$ \\
\hline & & 97.07 & $21.18 \%$ \\
\hline & $\begin{array}{l}\text { Two type of grains (UCO and UCO) in fuel } \\
\text { compact }\end{array}$ & 97.07 & $20.19 \%$ \\
\hline & One type of grain (UCO) in fuel compact & 97.07 & $10.27 \%$ \\
\hline \multirow{5}{*}{ Serpent } & \multirow{3}{*}{$\begin{array}{l}\text { Two type of grains }\left(\mathrm{UCO} \text { and } \mathrm{ThO}_{2}\right) \text { in fuel } \\
\text { compact }\end{array}$} & 1.01 & $0.10 \%$ \\
\hline & & 40.29 & $4.18 \%$ \\
\hline & & 97.08 & $10.04 \%$ \\
\hline & $\begin{array}{l}\text { Two type of grains (UCO and UCO) in fuel } \\
\text { compact }\end{array}$ & 97.08 & $10.11 \%$ \\
\hline & One type of grain (UCO) in fuel compact & 97.08 & 10.1 \\
\hline
\end{tabular}

Figure 3-10 shows the $\mathrm{k}_{\infty}$ values during depletion for the cases explained above and in Table $3-1$. The $\mathrm{k}_{\infty}$ values for the cases that have two different grain types show significant discrepancies while the case with a single grain type show very good agreement.
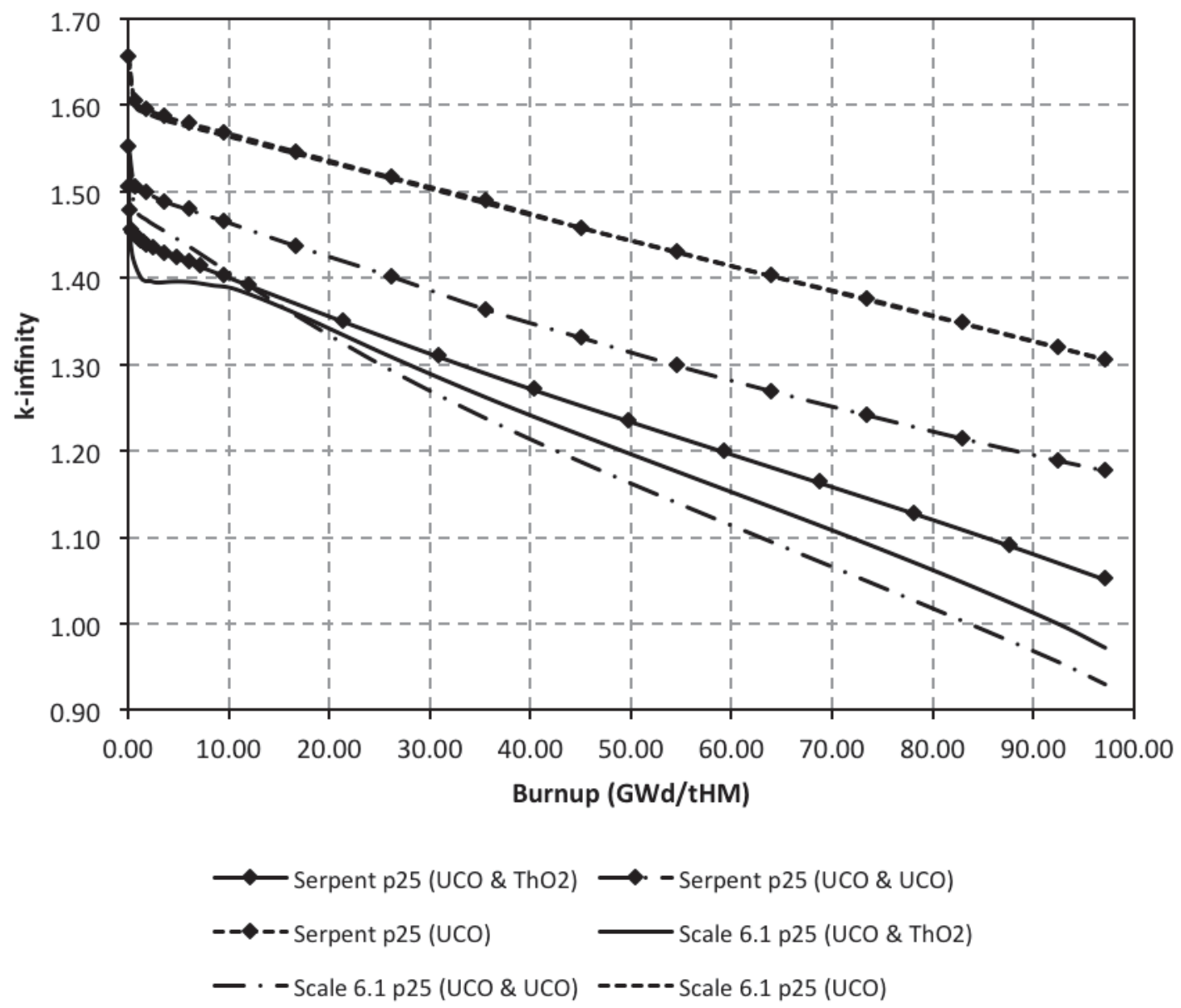

Figure 3-10: $\mathbf{k}_{\infty}$ for different models during depletion 


\subsection{Full Core Results}

As mentioned in section 2.2.1, the SCALE6.1 full core model did not converge for the transport calculation in a reasonable amount of time. Therefore, only Serpent full core results are presented in this section.

The burnable poison characteristics are not given in [1], and the size and the concentration are specified according to the reactivity and self-shielding requirements in each load. A simple attempt to identify the burnable poison characteristics was performed. Table 3-2 gives the characteristics of the lumped burnable poisons that were used in the analysis.

The unrodded hot $\mathrm{k}_{\text {eff }}$ for the equilibrium core is given in [1] to be 1.010 for the BOL, 1.025 for the MOL and 1.005 for the EOL. Figure 3-11 gives the calculated $\mathrm{k}_{\text {eff }}$ during depletion with the full core Serpent model. The effect of the self-shielding of the burnable poisons increases with increasing the diameter of the $\mathrm{B}_{4} \mathrm{C}$ kernel. Even though the cases that were studied in this report do not give the same $\mathrm{k}_{\text {eff }}$ results that were reported in [1], no further attempt was made to achieve similar results since it is outside of the scope of this work.

Table 3-2: Lumped burnable poison descriptions

\begin{tabular}{|l|r|r|r|}
\hline & Case (1) & Case (2) & Case (3) \\
\hline LBP holes per element & 6 & 6 & 6 \\
\hline Number of $B_{4}$ C particles per compact & 20,000 & 24,000 & 8,000 \\
\hline Volume fraction of $B_{4}$ C particles & 0.039 & 0.047 & 0.039 \\
\hline & 200 & 200 & 300 \\
\hline Kernel diameter $(\mu \mathrm{m})$ & 18 & 18 & 18 \\
\hline Buffer Coating thickness $(\mu \mathrm{m})$ & 23 & 23 & 23 \\
\hline Pyrolitic coating thickness $(\mu \mathrm{m})$ & 2.47 & 2.47 & 2.47 \\
\hline Kernel Density $\left(\mathrm{g} / \mathrm{cm}_{4}^{3}\right)$ & 1.00 & 1.00 & 1.00 \\
\hline Buffer Coating Density $\left(\mathrm{g} / \mathrm{cm}^{3}\right)$ & 1.87 & 1.87 & 1.87 \\
\hline Pyrolitic Coating Density $\left(\mathrm{g} / \mathrm{cm}^{3}\right)$ & 0.94 & 0.94 & 0.94 \\
\hline Matrix Graphite Density $\left(\mathrm{g} / \mathrm{cm}^{3}\right)$ & & & \\
\hline
\end{tabular}




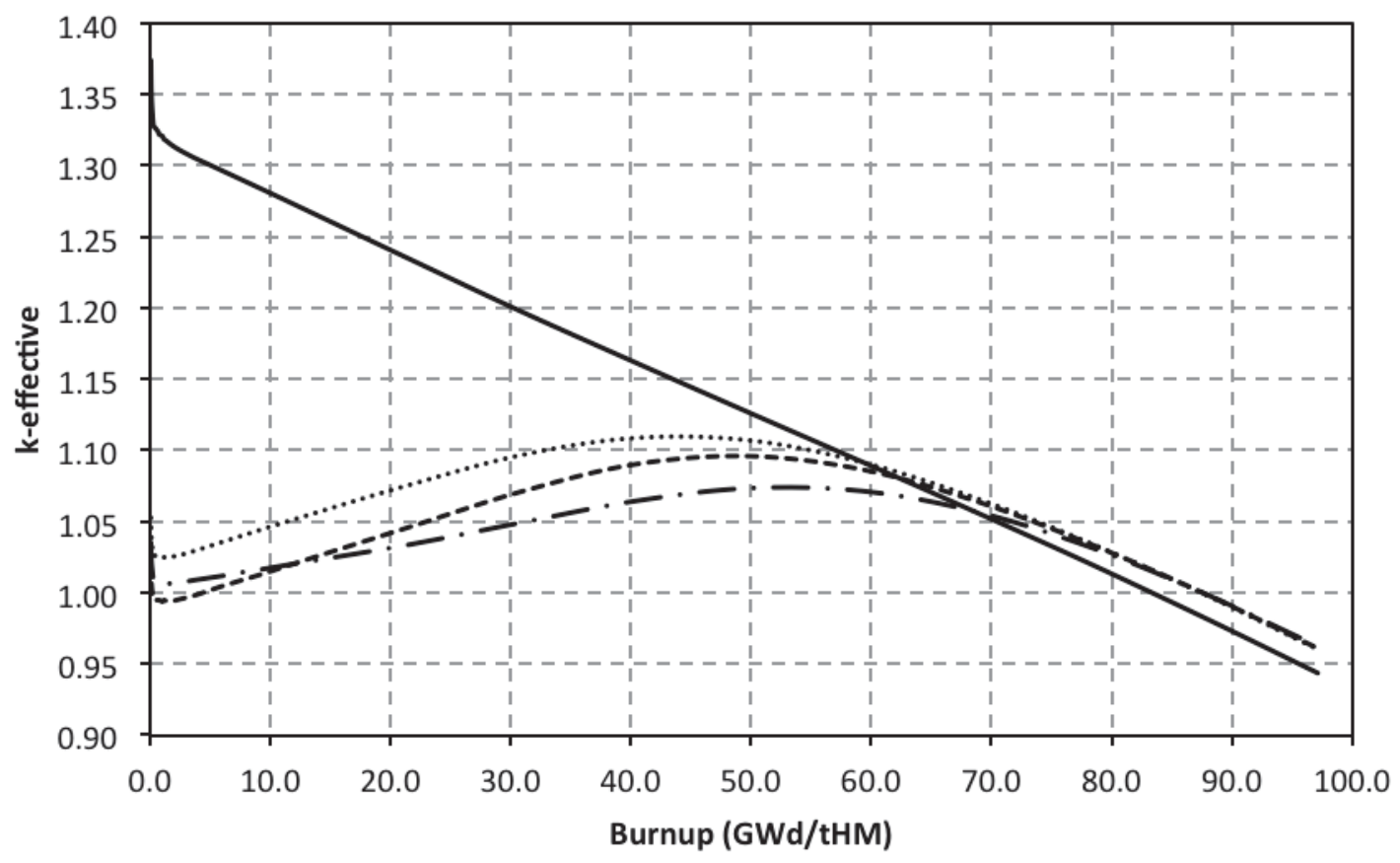

Case (1) -----Case (2) - - - Case (3) - no LBP

Figure 3-11: k-effective during depletion for the full core model

Figure 3-12 gives the mass fractions of U238 and Th232 during depletion for both unit cell and full core (with and without LBP) Serpent models, while Figure 3-13 gives the mass fraction of Pu239 and U233. Figure 3-14 to Figure 3-20 give the mass fractions of U235, U236, Np237, Pu240, Pu241, P242, and Am 242m, respectively. The results shown from Figure 3-13 to Figure 3-20 show good agreement between the unit cell and full core models. Table 3-3 gives the mass fraction of the actinides at the discharge burnup for 3 different Serpent models used in the analysis. The effect of the presence of LBP is not significant and for the purpose of this work only unit cell can be used. 

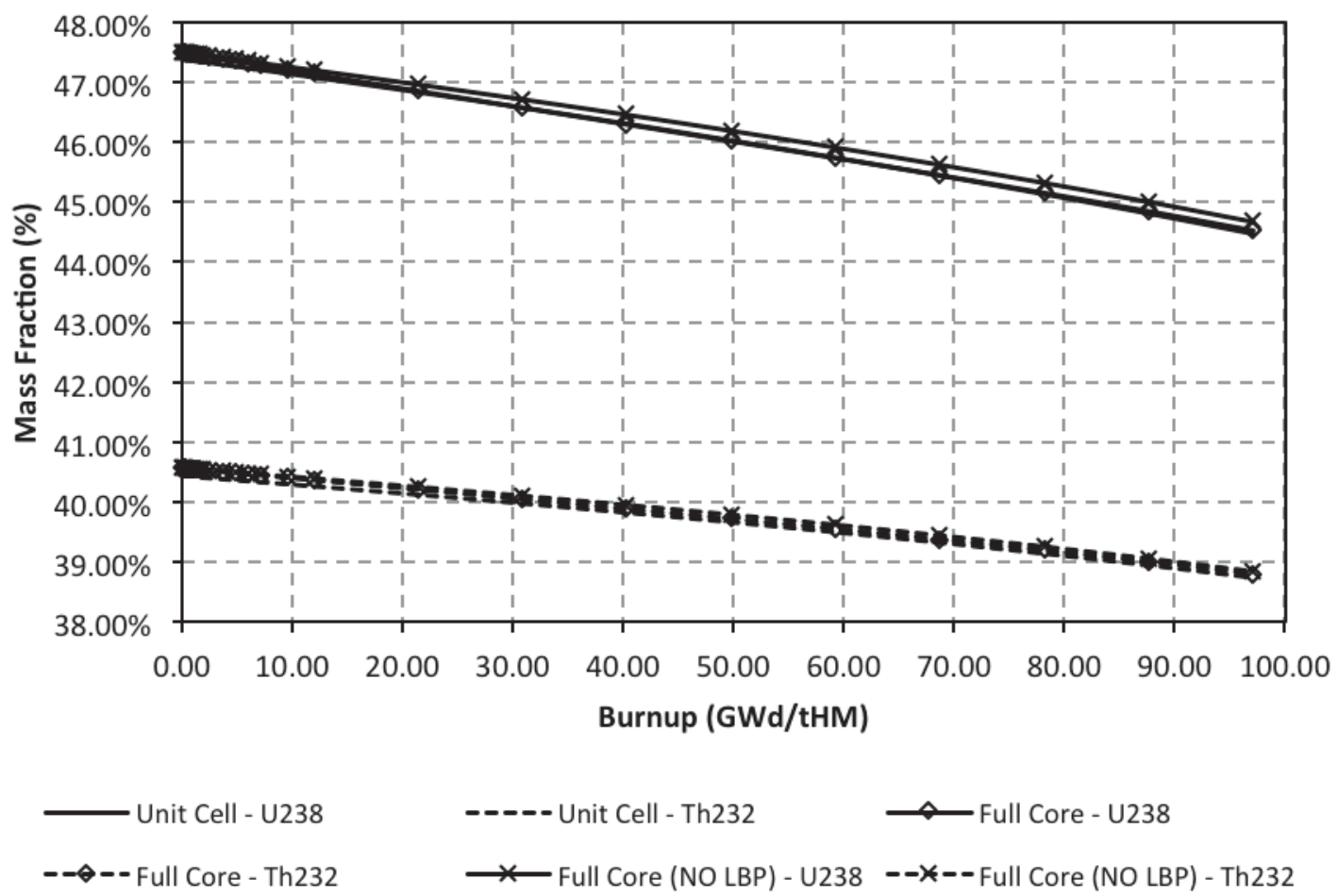

Figure 3-12: U238 and Th232 mass fractions during depletion

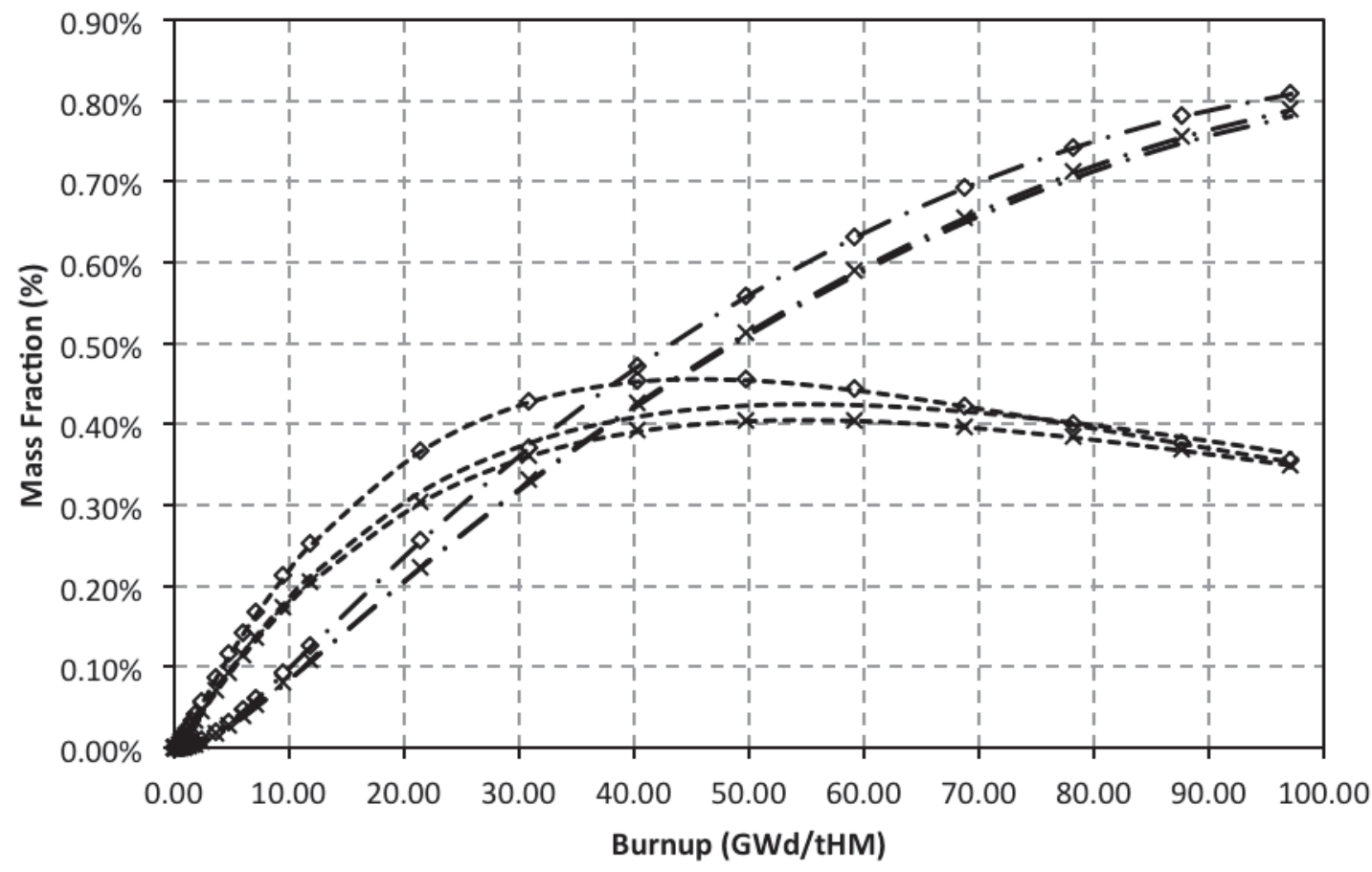

$$
\begin{array}{lll}
----- \text { Unit Cell - Pu239 } & -\cdot-\text { Unit Cell - U233 } & --\triangleright-- \text { Full Core - Pu239 } \\
\longrightarrow \text { - Full Core - U233 } & --x-- \text { Full Core (NO LBP) - Pu239 } \rightarrow \times \cdot-\text { Full Core (NO LBP) - U233 }
\end{array}
$$

Figure 3-13: U233 and Pu239 mass fractions during depletion 


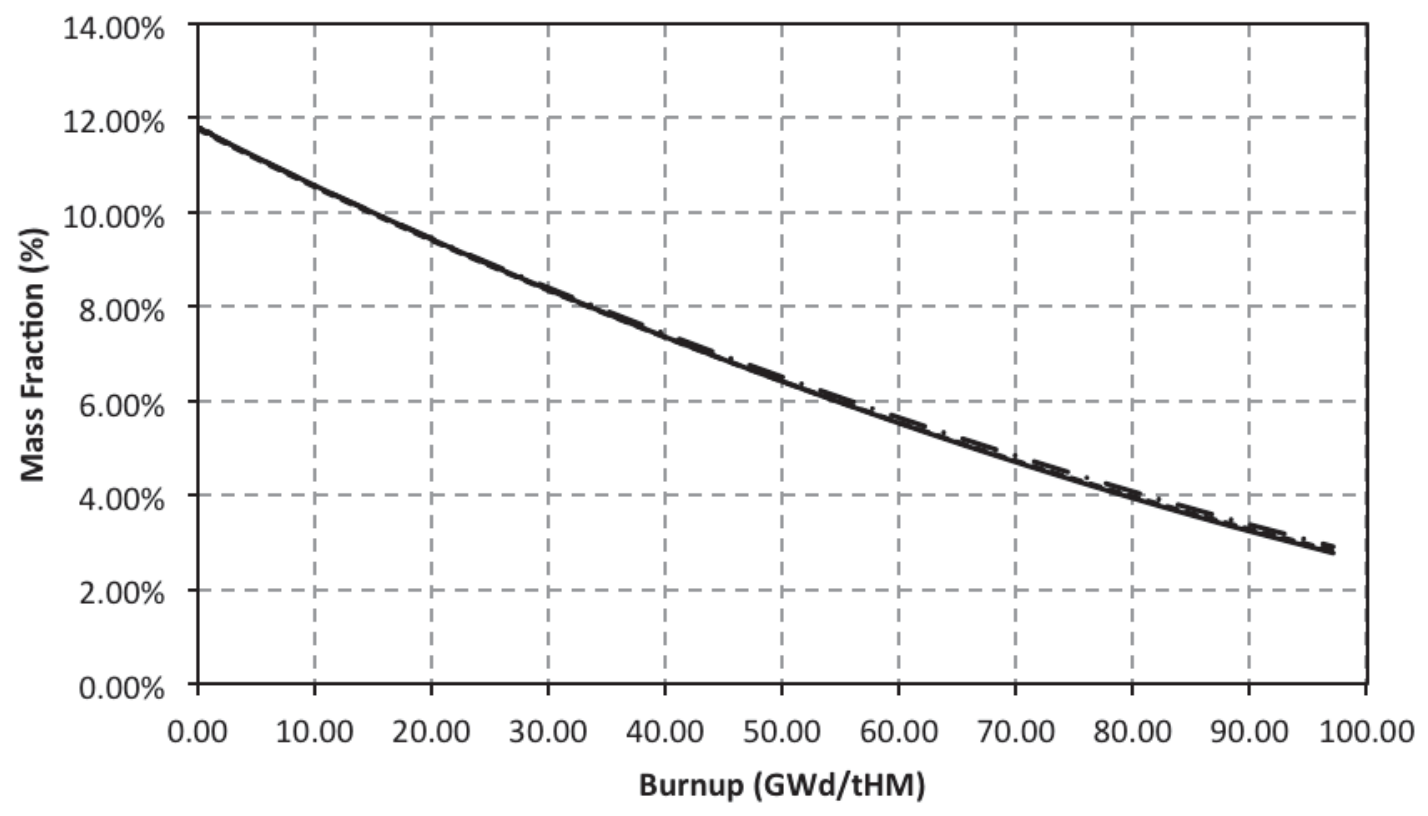

----Unit Cell - U235 - - Full Core - U235 — Full Core (NO LBP) - U235

Figure 3-14: U235 mass fraction during depletion

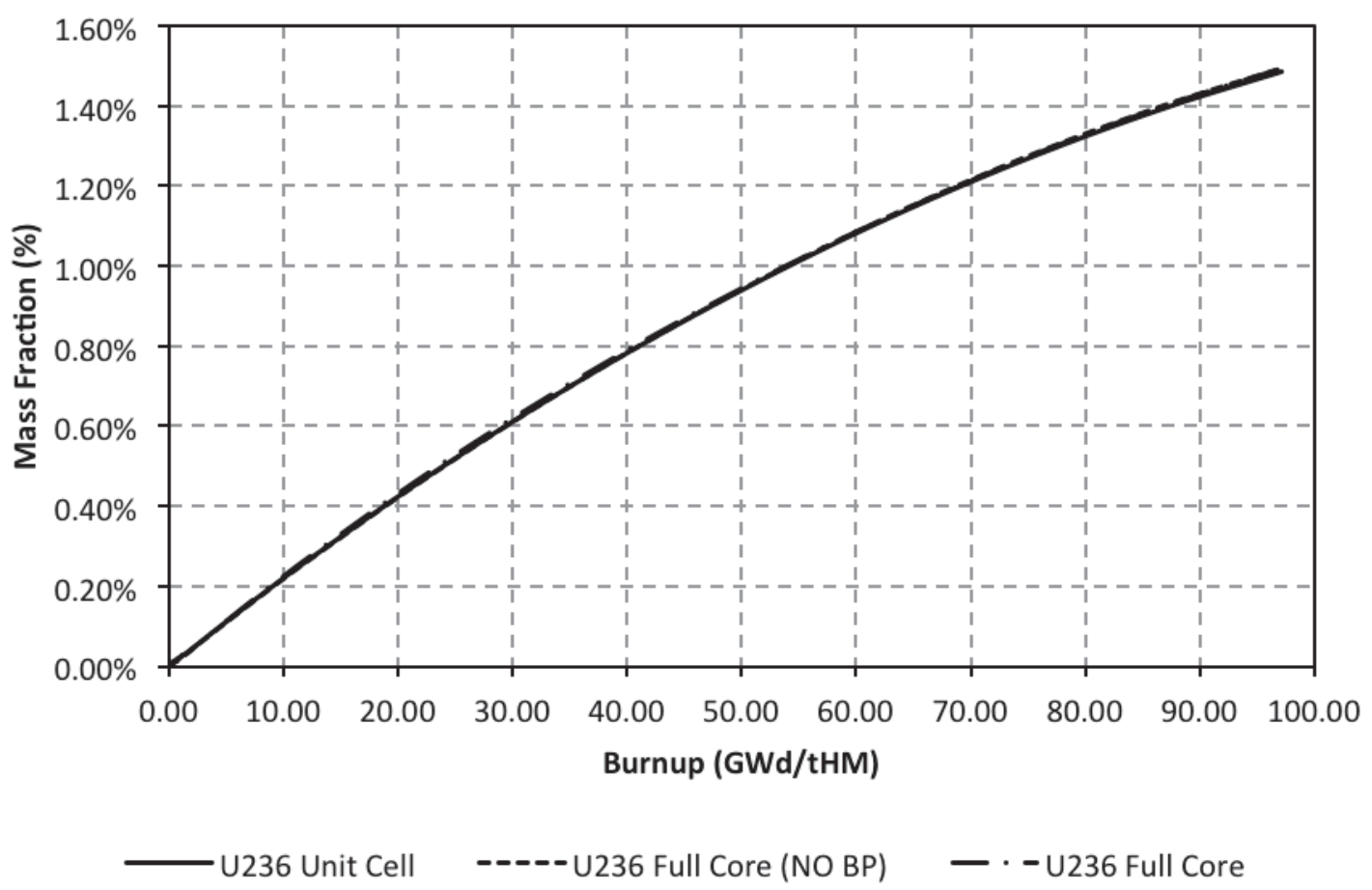

Figure 3-15: U236 mass fraction during depletion 


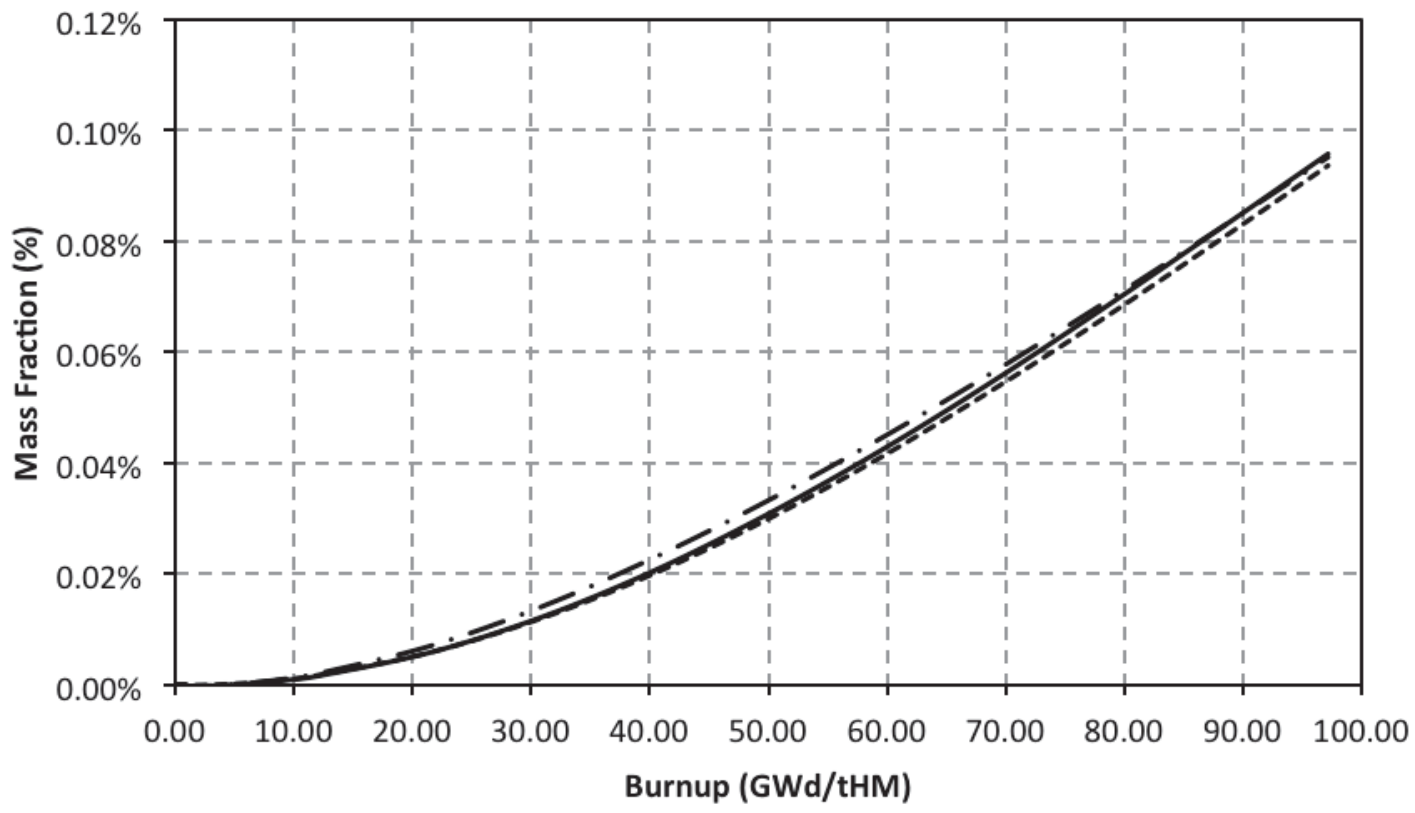

- Np237 Unit Cell _----Np237 Full Core (NO BP) — - Np237 Full Core

Figure 3-16: Np237 mass fraction during depletion

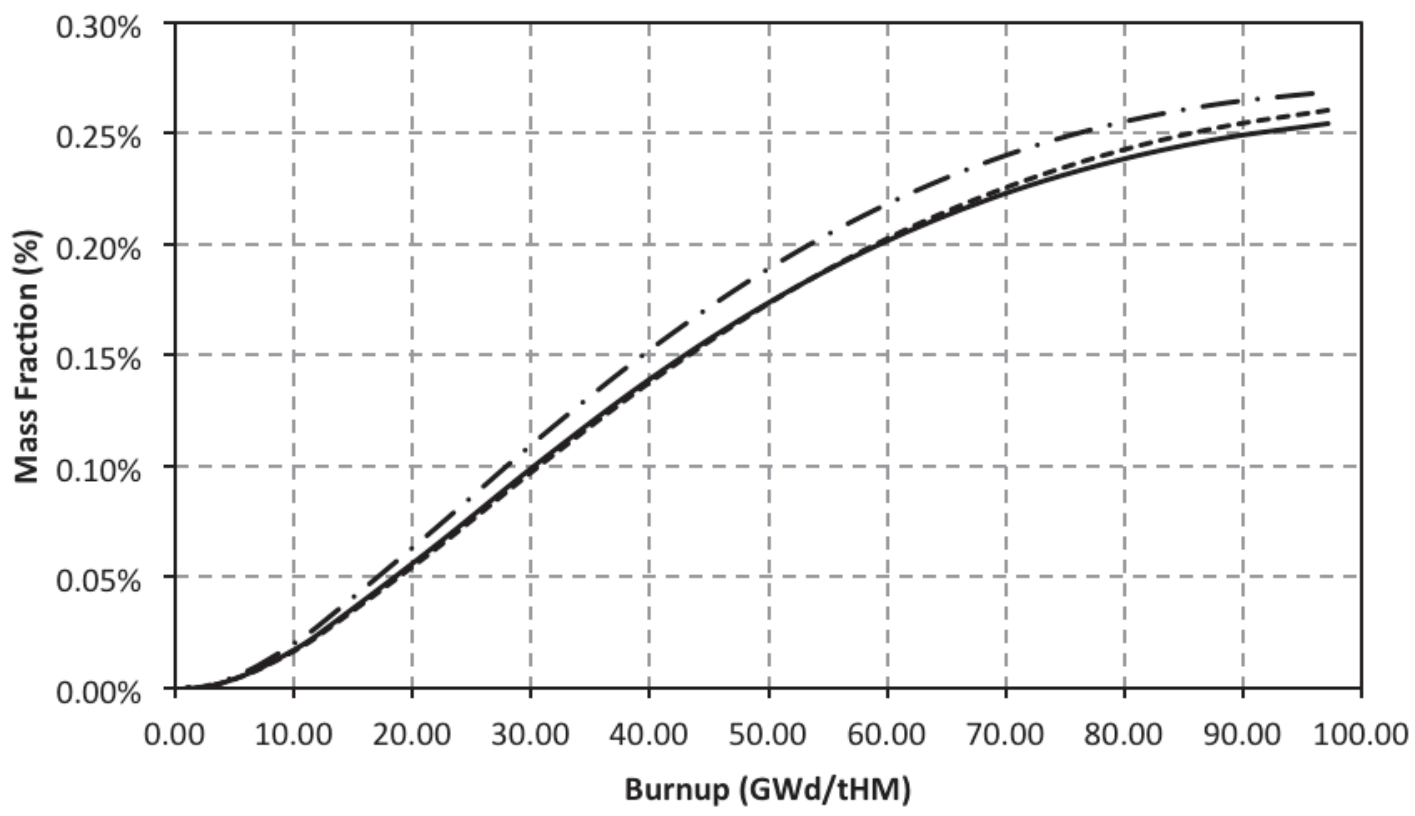

— Pu240 Unit Cell ----- Pu240 Full Core (NO BP) - - - Pu240 Full Core

Figure 3-17: Pu240 mass fraction during depletion 


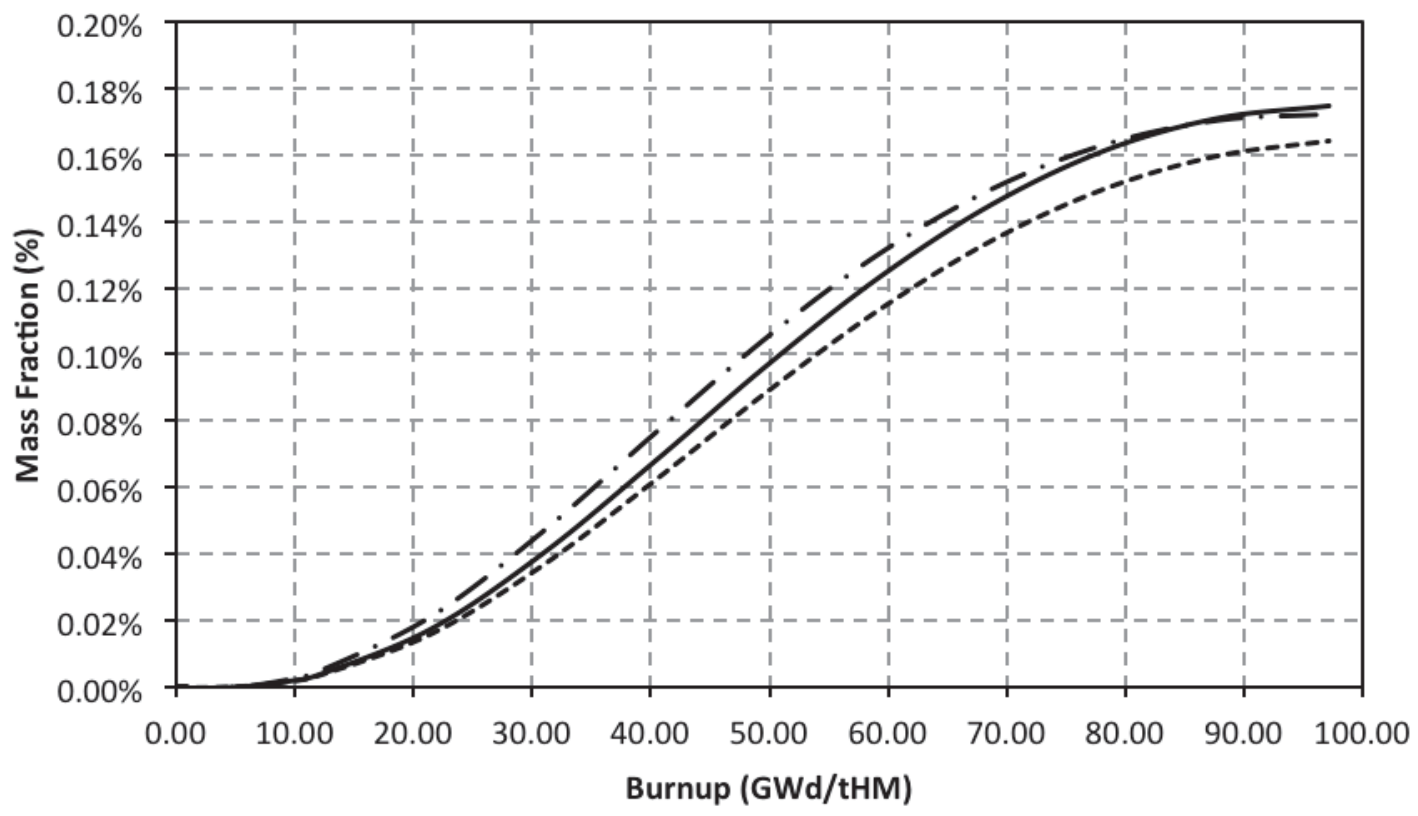

—-Pu241 Unit Cell ----- Pu241 Full Core (NO BP) - - - Pu241 Full Core

Figure 3-18: Pu241 mass fraction during depletion

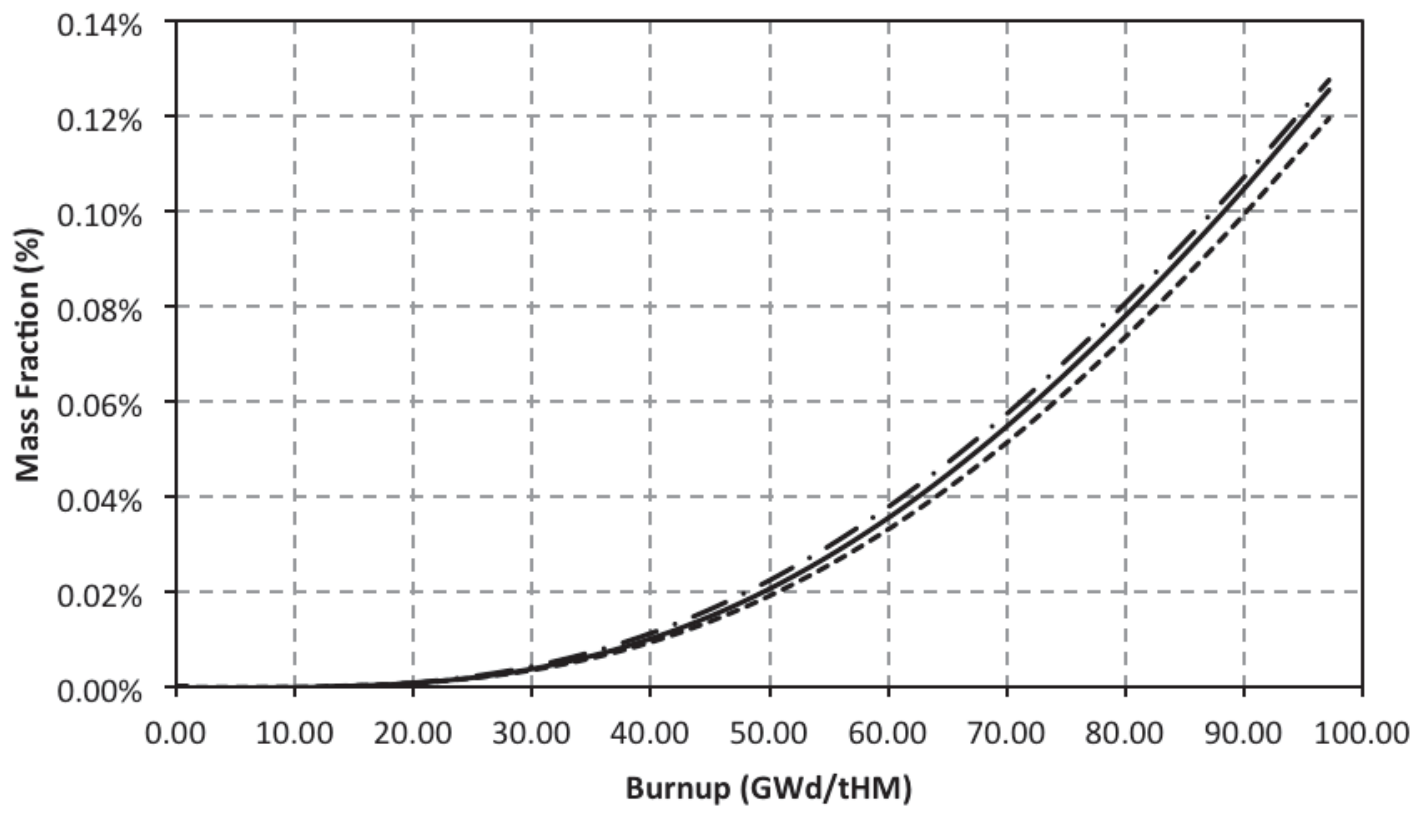

— Pu242 Unit Cell -----Pu242 Full Core (NO BP) - - - Pu242 Full Core

Figure 3-19: Pu242 mass fraction during depletion 


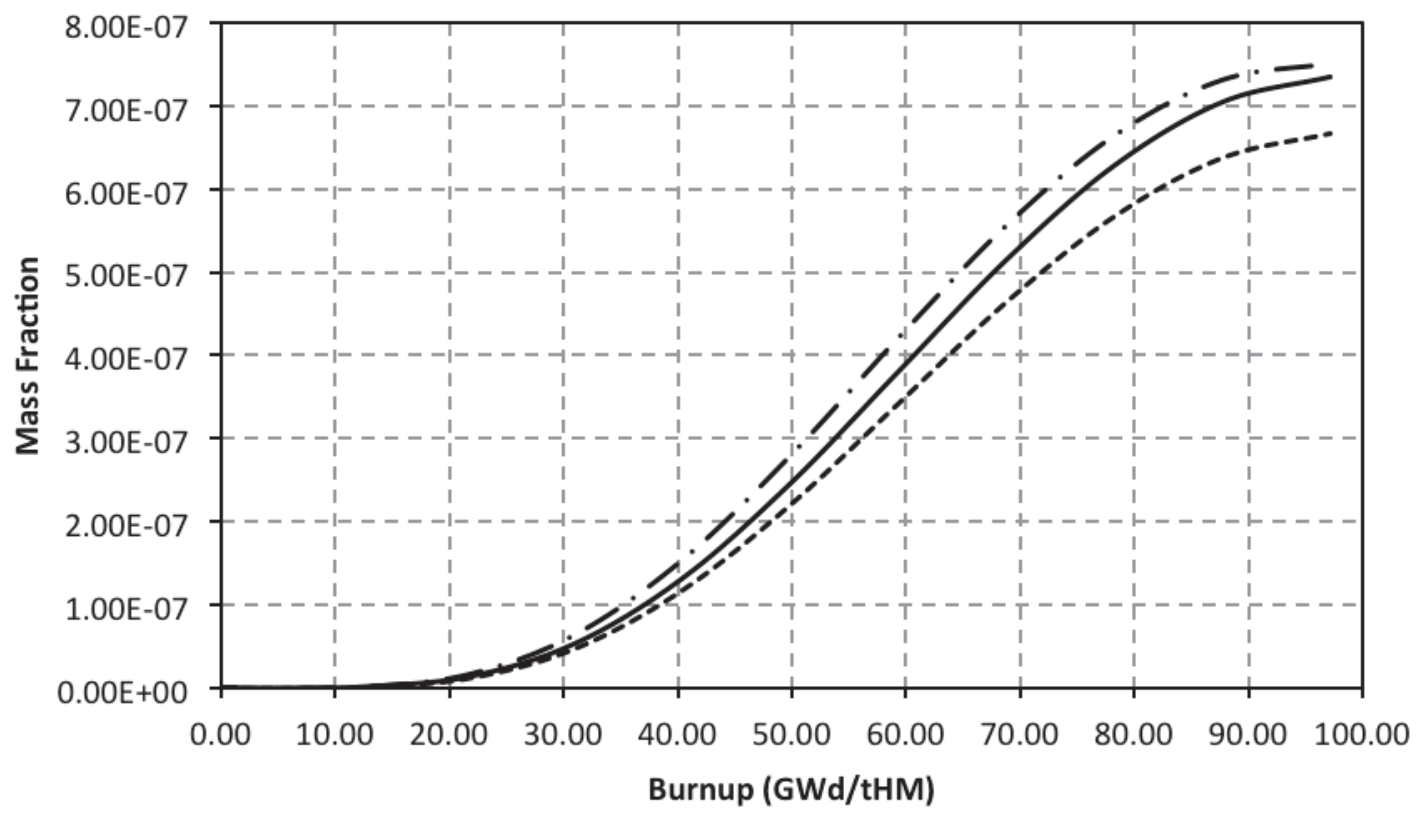

—Am242m Unit Cell ----Am242m Full Core (NO BP) - - Am242m Full Core

Figure 3-20: Am242m mass fraction during depletion

Table 3-3: Mass fractions of actinides at discharge for Serpent models

\begin{tabular}{|c|r|r|r|}
\hline \multirow{2}{*}{ Nuclide } & \multicolumn{3}{|c|}{ Mass Fraction } \\
\cline { 2 - 4 } & Unit Cell & Full Core (NO LBP) & Full Core (Case 3) \\
\hline \hline Th-232 & $3.8920 \mathrm{E}-01$ & $3.8923 \mathrm{E}-01$ & $3.8879 \mathrm{E}-01$ \\
\hline$P a-231$ & $1.9134 \mathrm{E}-06$ & $2.3664 \mathrm{E}-06$ & $2.4682 \mathrm{E}-06$ \\
\hline$U-232$ & $8.3564 \mathrm{E}-04$ & $8.5869 \mathrm{E}-04$ & $8.2775 \mathrm{E}-04$ \\
\hline$U-233$ & $2.1880 \mathrm{E}-06$ & $2.7012 \mathrm{E}-06$ & $2.9198 \mathrm{E}-06$ \\
\hline$U-234$ & $7.8503 \mathrm{E}-03$ & $7.9177 \mathrm{E}-03$ & $8.1098 \mathrm{E}-03$ \\
\hline$U-235$ & $9.9609 \mathrm{E}-04$ & $1.0015 \mathrm{E}-03$ & $1.0394 \mathrm{E}-03$ \\
\hline$U-236$ & $2.8564 \mathrm{E}-02$ & $2.7889 \mathrm{E}-02$ & $2.9263 \mathrm{E}-02$ \\
\hline$U-237$ & $1.4868 \mathrm{E}-02$ & $1.4961 \mathrm{E}-02$ & $1.4980 \mathrm{E}-02$ \\
\hline$U-238$ & $2.5984 \mathrm{E}-05$ & $2.5654 \mathrm{E}-05$ & $2.4878 \mathrm{E}-05$ \\
\hline$U-239$ & $4.4690 \mathrm{E}-01$ & $4.4773 \mathrm{E}-01$ & $4.4628 \mathrm{E}-01$ \\
\hline$N p-236$ & $8.4224 \mathrm{E}-07$ & $8.2699 \mathrm{E}-07$ & $7.9865 \mathrm{E}-07$ \\
\hline$N p-237$ & $2.4016 \mathrm{E}-10$ & $2.7876 \mathrm{E}-10$ & $2.9394 \mathrm{E}-10$ \\
\hline$N p-238$ & $9.6129 \mathrm{E}-04$ & $9.3814 \mathrm{E}-04$ & $9.5496 \mathrm{E}-04$ \\
\hline
\end{tabular}




\begin{tabular}{|c|c|c|c|}
\hline \multirow[b]{2}{*}{ Nuclide } & \multicolumn{3}{|c|}{ Mass Fraction } \\
\hline & Unit Cell & Full Core (NO LBP) & Full Core (Case 3) \\
\hline$N p-239$ & $1.2170 \mathrm{E}-04$ & $1.1949 \mathrm{E}-04$ & $1.1540 \mathrm{E}-04$ \\
\hline$P u-236$ & $2.6336 \mathrm{E}-12$ & $2.9249 \mathrm{E}-12$ & $3.2003 \mathrm{E}-12$ \\
\hline$P u-237$ & $4.7489 \mathrm{E}-11$ & $5.5609 \mathrm{E}-11$ & $5.9189 \mathrm{E}-11$ \\
\hline$P u-238$ & $3.0022 \mathrm{E}-04$ & $2.8749 \mathrm{E}-04$ & $2.9996 \mathrm{E}-04$ \\
\hline$P u-239$ & $3.6715 \mathrm{E}-03$ & $3.5112 \mathrm{E}-03$ & $3.5537 \mathrm{E}-03$ \\
\hline$P u-240$ & $2.5547 \mathrm{E}-03$ & $2.6136 \mathrm{E}-03$ & $2.6961 \mathrm{E}-03$ \\
\hline$P u-241$ & $1.7520 \mathrm{E}-03$ & $1.6452 \mathrm{E}-03$ & $1.7272 \mathrm{E}-03$ \\
\hline$P u-242$ & $1.2577 \mathrm{E}-03$ & $1.1970 \mathrm{E}-03$ & $1.2797 \mathrm{E}-03$ \\
\hline$P u-243$ & $2.7690 \mathrm{E}-07$ & $2.5650 \mathrm{E}-07$ & $2.6585 \mathrm{E}-07$ \\
\hline$P u-244$ & 4.0162E-08 & $3.5950 \mathrm{E}-08$ & $2.4143 \mathrm{E}-08$ \\
\hline$A m-241$ & $3.7553 \mathrm{E}-05$ & $3.4684 \mathrm{E}-05$ & 3.8819E-05 \\
\hline$A m-242$ & $1.8501 \mathrm{E}-07$ & $1.7297 \mathrm{E}-07$ & $1.8687 \mathrm{E}-07$ \\
\hline$A m-242 m$ & $7.3553 \mathrm{E}-07$ & $6.6793 \mathrm{E}-07$ & $7.5229 \mathrm{E}-07$ \\
\hline$A m-243$ & $1.8874 \mathrm{E}-04$ & $1.7232 \mathrm{E}-04$ & $1.8738 \mathrm{E}-04$ \\
\hline$A m-244$ & $7.3895 \mathrm{E}-09$ & $6.5757 \mathrm{E}-09$ & $1.3172 \mathrm{E}-07$ \\
\hline$A m-244 m$ & $6.0742 \mathrm{E}-09$ & $5.4065 \mathrm{E}-09$ & $2.9989 \mathrm{E}-10$ \\
\hline $\mathrm{Cm}-242$ & $2.5661 \mathrm{E}-05$ & $2.3834 \mathrm{E}-05$ & $2.6455 \mathrm{E}-05$ \\
\hline $\mathrm{Cm}-243$ & 4.3910E-07 & $4.1400 \mathrm{E}-07$ & $4.6308 \mathrm{E}-07$ \\
\hline $\mathrm{Cm}-244$ & 4.2947E-05 & $3.7461 \mathrm{E}-05$ & $4.1183 \mathrm{E}-05$ \\
\hline$C m-245$ & $1.5949 \mathrm{E}-06$ & $1.3578 \mathrm{E}-06$ & $1.5423 \mathrm{E}-06$ \\
\hline
\end{tabular}




\section{CONCLUSIONS.}

This report presents the results of a neutronics analysis in which the option of a LEU/Th based fuel cycle in high temperature reactors.

The main objective of these calculations is to quantify the isotopic inventory of a LEU/Th based MHTGR at the discharge burnup. During the analysis some problems were observed with the use of Scale6.1, therefore Serpent code were used for the final results. A full core and a unit cell model were developed. Unit cell model have been adjusted to have a similar spectrum indices (capture to fission ratio) as the full core model. A simple attempt to identify the burnable poison design was made, however an optimum design has not been achieved. The final results showed that the use of unit cell for quantifying the isotopic inventory at the discharge burnup is adequate.

It is important to recognize that these results of LEU/Th based MHTGR are based on the information available in literature. It does not represent the optimum performance achievable. 


\section{REFERENCES.}

1. MHTGR Preliminary Safety Information Document, DOE-HTGR-86-024, General Atomics, 1995.

2. L.Gauld, O. Herman, et al., "Origen-S: Scale system module to calculate fuel depletion, Actinide transmutation, fission product buildup and decay, and associated radiation source terms", Oak Ridge National Laboratory (2009).

3. M. DeHart, "Lattice Physics Capabilities of the SCALE Code System Using TRITON", Physor Conference (2006).

4. Leppänen, J. "PSG2/Serpent - A Continuous-energy Monte Carlo Reactor Physics Burnup Calculation Code: User's Manual," VTT Technical Research Centre of Finland, available at http://montecarlo.vtt.fi, August 16, 2012.

5. Massimo, L., Physics of High Temperature Reactors, Elsevier Science and Technology Books, 1976.

6. M.A. Pope, "Transmutation Analysis of Enriched Uranium and Deep Burn High Temperature Reactors”, Idaho National Laboratory, INL/EXT-12-26423 (2012).

7. M.A. Jessee, M.D. DeHart, "TRITON: A Multipurpose Transport, Depletion, and Sensitivity and Uncertainity Analysis Module", Oak Ridge National Laboratory, ORNL/TM-2005/39 Version 6.1, Sect. T1 (2011). 


\section{Appendix 1: Detailed isotopic masses (grams per initial ton HM) in fresh and discharged fuel for the Serpent Models.}

\begin{tabular}{|c|c|c|c|c|c|c|}
\hline \multirow[b]{2}{*}{ Nuclide } & \multicolumn{3}{|c|}{ Fresh Fuel } & \multicolumn{3}{|c|}{ Discharge } \\
\hline & Unit Cell & $\begin{array}{l}\text { Full Core } \\
\text { (NO LBP) }\end{array}$ & Full Core (3) & Unit Cell & $\begin{array}{l}\text { Full Core } \\
\text { (NO LBP) }\end{array}$ & Full Core (3) \\
\hline $\mathrm{He}-6$ & $0.00000 \mathrm{E}+00$ & $0.00000 \mathrm{E}+00$ & $0.00000 \mathrm{E}+00$ & $3.72515 \mathrm{E}-31$ & $8.38446 \mathrm{E}-31$ & $1.06505 \mathrm{E}-30$ \\
\hline$L i-6$ & $0.00000 \mathrm{E}+00$ & $0.00000 \mathrm{E}+00$ & $0.00000 \mathrm{E}+00$ & $3.63867 \mathrm{E}-24$ & $8.10504 \mathrm{E}-24$ & $1.10796 \mathrm{E}-23$ \\
\hline$L i-8$ & $0.00000 \mathrm{E}+00$ & $0.00000 \mathrm{E}+00$ & $0.00000 \mathrm{E}+00$ & $1.19942 \mathrm{E}-29$ & $1.94826 \mathrm{E}-29$ & $1.85511 \mathrm{E}-29$ \\
\hline$L i-9$ & $0.00000 \mathrm{E}+00$ & $0.00000 \mathrm{E}+00$ & $0.00000 \mathrm{E}+00$ & $0.00000 \mathrm{E}+00$ & $0.00000 \mathrm{E}+00$ & $0.00000 \mathrm{E}+00$ \\
\hline $\mathrm{Be}-8$ & $0.00000 \mathrm{E}+00$ & $0.00000 \mathrm{E}+00$ & $0.00000 \mathrm{E}+00$ & $0.00000 \mathrm{E}+00$ & $0.00000 \mathrm{E}+00$ & $0.00000 \mathrm{E}+00$ \\
\hline$B e-10$ & $0.00000 \mathrm{E}+00$ & $0.00000 \mathrm{E}+00$ & $0.00000 \mathrm{E}+00$ & $4.88780 \mathrm{E}-24$ & $9.09589 \mathrm{E}-24$ & $1.22070 \mathrm{E}-23$ \\
\hline$B e-11$ & $0.00000 \mathrm{E}+00$ & $0.00000 \mathrm{E}+00$ & $0.00000 \mathrm{E}+00$ & $4.65560 \mathrm{E}-30$ & $7.15894 \mathrm{E}-30$ & $6.69423 \mathrm{E}-30$ \\
\hline$B-12$ & $0.00000 \mathrm{E}+00$ & $0.00000 \mathrm{E}+00$ & $0.00000 \mathrm{E}+00$ & $3.52649 \mathrm{E}-21$ & $5.16693 \mathrm{E}-21$ & $67326 \mathrm{E}-21$ \\
\hline$C-13$ & $0.00000 \mathrm{E}+00$ & $0.00000 \mathrm{E}+00$ & $0.00000 \mathrm{E}+00$ & $7.21365 \mathrm{E}-01$ & $8.67386 \mathrm{E}-01$ & $30633 \mathrm{E}-01$ \\
\hline$C-14$ & $0.00000 \mathrm{E}+00$ & $0.00000 \mathrm{E}+00$ & $0.00000 \mathrm{E}+00$ & $1.55277 \mathrm{E}-05$ & $1.66612 \mathrm{E}-05$ & $1.56599 \mathrm{E}-05$ \\
\hline$C-15$ & $0.00000 \mathrm{E}+00$ & $0.00000 \mathrm{E}+00$ & $0.00000 \mathrm{E}+00$ & $1.01779 \mathrm{E}-19$ & $1.47464 \mathrm{E}-19$ & $1.61693 \mathrm{E}-19$ \\
\hline $0-18$ & $0.00000 \mathrm{E}+00$ & $0.00000 \mathrm{E}+00$ & $0.00000 \mathrm{E}+00$ & $2.56049 \mathrm{E}-07$ & $2.64475 \mathrm{E}-07$ & $2.45496 \mathrm{E}-07$ \\
\hline$M g-25$ & $0.00000 \mathrm{E}+00$ & $0.00000 \mathrm{E}+00$ & $0.00000 \mathrm{E}+00$ & $0.00000 \mathrm{E}+00$ & $0.00000 \mathrm{E}+00$ & $0.00000 \mathrm{E}+00$ \\
\hline$M g-26$ & $0.00000 \mathrm{E}+00$ & $0.00000 \mathrm{E}+00$ & $0.00000 \mathrm{E}+00$ & $0.00000 \mathrm{E}+00$ & $0.00000 \mathrm{E}+00$ & $0.00000 \mathrm{E}+00$ \\
\hline$M g-27$ & $0.00000 \mathrm{E}+00$ & $0.00000 \mathrm{E}+00$ & $0.00000 \mathrm{E}+00$ & $0.00000 \mathrm{E}+00$ & $0.00000 \mathrm{E}+00$ & $0.00000 \mathrm{E}+00$ \\
\hline Al-27 & $0.00000 \mathrm{E}+00$ & $0.00000 \mathrm{E}+00$ & $0.00000 \mathrm{E}+00$ & $0.00000 \mathrm{E}+00$ & $0.00000 \mathrm{E}+00$ & $0.00000 \mathrm{E}+00$ \\
\hline Al-28 & $0.00000 \mathrm{E}+00$ & $0.00000 \mathrm{E}+00$ & $0.00000 \mathrm{E}+00$ & $0.00000 \mathrm{E}+00$ & $0.00000 \mathrm{E}+00$ & $0.00000 \mathrm{E}+00$ \\
\hline Al-29 & $0.00000 \mathrm{E}+00$ & $0.00000 \mathrm{E}+00$ & $0.00000 \mathrm{E}+00$ & $0.00000 \mathrm{E}+00$ & $0.00000 \mathrm{E}+00$ & $0.00000 \mathrm{E}+00$ \\
\hline Al-30 & $0.00000 \mathrm{E}+00$ & $0.00000 \mathrm{E}+00$ & $0.00000 \mathrm{E}+00$ & $0.00000 \mathrm{E}+00$ & $0.00000 \mathrm{E}+00$ & $0.00000 \mathrm{E}+00$ \\
\hline$S i-31$ & $0.00000 \mathrm{E}+00$ & $0.00000 \mathrm{E}+00$ & $0.00000 \mathrm{E}+00$ & $0.00000 \mathrm{E}+00$ & $0.00000 \mathrm{E}+00$ & $0.00000 \mathrm{E}+00$ \\
\hline$P-31$ & $0.00000 \mathrm{E}+00$ & $0.00000 \mathrm{E}+00$ & $0.00000 \mathrm{E}+00$ & $0.00000 \mathrm{E}+00$ & $0.00000 \mathrm{E}+00$ & $0.00000 \mathrm{E}+00$ \\
\hline $\mathrm{Cr}-66$ & $0.00000 \mathrm{E}+00$ & $0.00000 \mathrm{E}+00$ & $0.00000 \mathrm{E}+00$ & $2.65712 \mathrm{E}-19$ & $3.11942 \mathrm{E}-19$ & $3.06943 \mathrm{E}-19$ \\
\hline $\mathrm{Cr}-67$ & $0.00000 \mathrm{E}+00$ & $0.00000 \mathrm{E}+00$ & $0.00000 \mathrm{E}+00$ & $3.38246 \mathrm{E}-19$ & $4.18810 \mathrm{E}-19$ & $4.06760 \mathrm{E}-19$ \\
\hline$M n-66$ & $0.00000 \mathrm{E}+00$ & $0.00000 \mathrm{E}+00$ & $0.00000 \mathrm{E}+00$ & $3.78363 \mathrm{E}-16$ & $4.24039 \mathrm{E}-16$ & 4.21952E-16 \\
\hline$M n-67$ & $0.00000 \mathrm{E}+00$ & $0.00000 \mathrm{E}+00$ & $0.00000 \mathrm{E}+00$ & $2.19617 \mathrm{E}-16$ & $2.52080 \mathrm{E}-16$ & $2.48074 \mathrm{E}-16$ \\
\hline$M n-68$ & $0.00000 \mathrm{E}+00$ & $0.00000 \mathrm{E}+00$ & $0.00000 \mathrm{E}+00$ & $2.25380 \mathrm{E}-17$ & $2.62331 \mathrm{E}-17$ & $2.57177 \mathrm{E}-17$ \\
\hline Mn-69 & $0.00000 \mathrm{E}+00$ & $0.00000 \mathrm{E}+00$ & $0.00000 \mathrm{E}+00$ & $1.45286 \mathrm{E}-18$ & $1.68288 \mathrm{E}-18$ & $1.64935 \mathrm{E}-18$ \\
\hline $\mathrm{Fe}-66$ & $0.00000 \mathrm{E}+00$ & $0.00000 \mathrm{E}+00$ & $0.00000 \mathrm{E}+00$ & $1.29785 \mathrm{E}-13$ & $1.39944 \mathrm{E}-13$ & $1.40710 \mathrm{E}-13$ \\
\hline $\mathrm{Fe}-67$ & $0.00000 \mathrm{E}+00$ & $0.00000 \mathrm{E}+00$ & $0.00000 \mathrm{E}+00$ & $1.78483 \mathrm{E}-13$ & $1.91169 \mathrm{E}-13$ & $1.90544 \mathrm{E}-13$ \\
\hline $\mathrm{Fe}-68$ & $0.00000 \mathrm{E}+00$ & $0.00000 \mathrm{E}+00$ & $0.00000 \mathrm{E}+00$ & $4.32050 \mathrm{E}-14$ & $4.60346 \mathrm{E}-14$ & $4.57675 \mathrm{E}-14$ \\
\hline $\mathrm{Fe}-69$ & $0.00000 \mathrm{E}+00$ & $0.00000 \mathrm{E}+00$ & $0.00000 \mathrm{E}+00$ & $8.76863 \mathrm{E}-15$ & $9.32719 \mathrm{E}-15$ & $9.25400 \mathrm{E}-15$ \\
\hline
\end{tabular}




\begin{tabular}{|c|c|c|c|c|c|c|}
\hline \multirow[b]{2}{*}{ Nuclide } & \multicolumn{3}{|c|}{ Fresh Fuel } & \multicolumn{3}{|c|}{ Discharge } \\
\hline & Unit Cell & $\begin{array}{l}\text { Full Core } \\
\text { (NO LBP) }\end{array}$ & Full Core (3) & Unit Cell & $\begin{array}{l}\text { Full Core } \\
(N O L B P)\end{array}$ & Full Core (3) \\
\hline $\mathrm{Fe}-70$ & $0.00000 \mathrm{E}+00$ & $0.00000 \mathrm{E}+00$ & $0.00000 \mathrm{E}+00$ & $2.42422 \mathrm{E}-15$ & $2.55690 \mathrm{E}-15$ & $2.53441 \mathrm{E}-15$ \\
\hline $\mathrm{Fe}-71$ & $0.00000 \mathrm{E}+00$ & $0.00000 \mathrm{E}+00$ & $0.00000 \mathrm{E}+00$ & $5.66363 \mathrm{E}-16$ & $5.95268 \mathrm{E}-16$ & $5.89670 \mathrm{E}-16$ \\
\hline $\mathrm{Fe}-72$ & $0.00000 \mathrm{E}+00$ & $0.00000 \mathrm{E}+00$ & $0.00000 \mathrm{E}+00$ & $5.65176 \mathrm{E}-17$ & $5.95200 \mathrm{E}-17$ & $5.89738 \mathrm{E}-17$ \\
\hline Co-66 & $0.00000 \mathrm{E}+00$ & $0.00000 \mathrm{E}+00$ & $0.00000 \mathrm{E}+00$ & $1.82468 \mathrm{E}-13$ & $1.97210 \mathrm{E}-13$ & $1.98747 \mathrm{E}-13$ \\
\hline Co-67 & $0.00000 \mathrm{E}+00$ & $0.00000 \mathrm{E}+00$ & $0.00000 \mathrm{E}+00$ & 7.94714E-13 & $8.39248 \mathrm{E}-13$ & $8.39052 \mathrm{E}-13$ \\
\hline Co-68 & $0.00000 \mathrm{E}+00$ & $0.00000 \mathrm{E}+00$ & $0.00000 \mathrm{E}+00$ & $4.46762 \mathrm{E}-13$ & $4.65598 \mathrm{E}-13$ & $4.63718 \mathrm{E}-13$ \\
\hline Co-69 & $0.00000 \mathrm{E}+00$ & $0.00000 \mathrm{E}+00$ & $0.00000 \mathrm{E}+00$ & $6.08749 \mathrm{E}-13$ & $6.27527 \mathrm{E}-13$ & $6.23582 \mathrm{E}-13$ \\
\hline Co-70 & $0.00000 \mathrm{E}+00$ & $0.00000 \mathrm{E}+00$ & $0.00000 \mathrm{E}+00$ & $2.63295 \mathrm{E}-13$ & $2.69841 \mathrm{E}-13$ & $2.67726 \mathrm{E}-13$ \\
\hline Co-71 & $0.00000 \mathrm{E}+00$ & $0.00000 \mathrm{E}+00$ & $0.00000 \mathrm{E}+00$ & $1.39986 \mathrm{E}-13$ & $1.43590 \mathrm{E}-13$ & $1.42498 \mathrm{E}-13$ \\
\hline Co-72 & $0.00000 \mathrm{E}+00$ & $0.00000 \mathrm{E}+00$ & $0.00000 \mathrm{E}+00$ & $3.30483 \mathrm{E}-14$ & $3.40503 \mathrm{E}-14$ & $3.37859 \mathrm{E}-14$ \\
\hline Co-73 & $0.00000 \mathrm{E}+00$ & $0.00000 \mathrm{E}+00$ & $0.00000 \mathrm{E}+00$ & $9312 \mathrm{E}-15$ & $6.61821 \mathrm{E}-15$ & $7883 \mathrm{E}-15$ \\
\hline Co- 74 & $0.00000 \mathrm{E}+00$ & $0.00000 \mathrm{E}+00$ & $0.00000 \mathrm{E}+00$ & $6.31863 \mathrm{E}-16$ & $6.55620 \mathrm{E}-16$ & $6.52426 \mathrm{E}-16$ \\
\hline Co- 75 & $0.00000 \mathrm{E}+00$ & $0.00000 \mathrm{E}+00$ & $0.00000 \mathrm{E}+00$ & $7.78742 \mathrm{E}-17$ & $8.09186 \mathrm{E}-17$ & $8.05473 \mathrm{E}-17$ \\
\hline $\mathrm{Ni}-66$ & $0.00000 \mathrm{E}+00$ & $0.00000 \mathrm{E}+00$ & $0.00000 \mathrm{E}+00$ & $2.01167 \mathrm{E}-07$ & $2.18224 \mathrm{E}-07$ & $2.20185 \mathrm{E}-07$ \\
\hline $\mathrm{Ni}-67$ & $0.00000 \mathrm{E}+00$ & $0.00000 \mathrm{E}+00$ & $0.00000 \mathrm{E}+00$ & $6.38922 \mathrm{E}-11$ & $6.73810 \mathrm{E}-11$ & $6.74422 \mathrm{E}-11$ \\
\hline $\mathrm{Ni}-68$ & $0.00000 \mathrm{E}+00$ & $0.00000 \mathrm{E}+00$ & $0.00000 \mathrm{E}+00$ & $2.13673 \mathrm{E}-10$ & $2.20764 \mathrm{E}-10$ & $2.19937 \mathrm{E}-10$ \\
\hline $\mathrm{Ni}-69$ & $0.00000 \mathrm{E}+00$ & $0.00000 \mathrm{E}+00$ & $0.00000 \mathrm{E}+00$ & $1.93774 \mathrm{E}-10$ & $1.97532 \mathrm{E}-10$ & $1.96128 \mathrm{E}-10$ \\
\hline $\mathrm{Ni}-70$ & $0.00000 \mathrm{E}+00$ & $0.00000 \mathrm{E}+00$ & $0.00000 \mathrm{E}+00$ & $2.63044 \mathrm{E}-10$ & $2.66634 \mathrm{E}-10$ & $2.64252 \mathrm{E}-10$ \\
\hline $\mathrm{Ni}-71$ & $0.00000 \mathrm{E}+00$ & $0.00000 \mathrm{E}+00$ & $0.00000 \mathrm{E}+00$ & $1.94058 \mathrm{E}-10$ & $1.97408 \mathrm{E}-10$ & $1.95795 \mathrm{E}-10$ \\
\hline $\mathrm{Ni}-72$ & $0.00000 \mathrm{E}+00$ & $0.00000 \mathrm{E}+00$ & $0.00000 \mathrm{E}+00$ & $1.35458 \mathrm{E}-10$ & $1.38189 \mathrm{E}-10$ & $1.37042 \mathrm{E}-10$ \\
\hline $\mathrm{Ni}-73$ & $0.00000 \mathrm{E}+00$ & $0.00000 \mathrm{E}+00$ & $0.00000 \mathrm{E}+00$ & $4.68658 \mathrm{E}-11$ & $4.78370 \mathrm{E}-11$ & 4.75693E-11 \\
\hline $\mathrm{Ni}-74$ & $0.00000 \mathrm{E}+00$ & $0.00000 \mathrm{E}+00$ & $0.00000 \mathrm{E}+00$ & $1.85826 \mathrm{E}-11$ & $1.89413 \mathrm{E}-11$ & $1.88817 \mathrm{E}-11$ \\
\hline $\mathrm{Ni}-75$ & $0.00000 \mathrm{E}+00$ & $0.00000 \mathrm{E}+00$ & $0.00000 \mathrm{E}+00$ & $5.18957 \mathrm{E}-12$ & $5.29510 \mathrm{E}-12$ & $5.28315 \mathrm{E}-12$ \\
\hline $\mathrm{Ni}-76$ & $0.00000 \mathrm{E}+00$ & $0.00000 \mathrm{E}+00$ & $0.00000 \mathrm{E}+00$ & $5.06471 \mathrm{E}-13$ & $5.16516 \mathrm{E}-13$ & 5.17354E-13 \\
\hline $\mathrm{Ni}-77$ & $0.00000 \mathrm{E}+00$ & $0.00000 \mathrm{E}+00$ & $0.00000 \mathrm{E}+00$ & $2.83490 \mathrm{E}-14$ & $2.90190 \mathrm{E}-14$ & 90966E-14 \\
\hline $\mathrm{Ni}-78$ & $0.00000 \mathrm{E}+00$ & $0.00000 \mathrm{E}+00$ & $0.00000 \mathrm{E}+00$ & $2.50345 \mathrm{E}-15$ & $2.59285 \mathrm{E}-15$ & $2.59710 \mathrm{E}-15$ \\
\hline $\mathrm{Cu}-66$ & $0.00000 \mathrm{E}+00$ & $0.00000 \mathrm{E}+00$ & $0.00000 \mathrm{E}+00$ & $3.15656 \mathrm{E}-10$ & $3.42464 \mathrm{E}-10$ & $3.45551 \mathrm{E}-10$ \\
\hline $\mathrm{Cu}-67$ & $0.00000 \mathrm{E}+00$ & $0.00000 \mathrm{E}+00$ & $0.00000 \mathrm{E}+00$ & $6.88962 \mathrm{E}-07$ & $7.26586 \mathrm{E}-07$ & $7.27416 \mathrm{E}-07$ \\
\hline Cu-68 & $0.00000 \mathrm{E}+00$ & $0.00000 \mathrm{E}+00$ & $0.00000 \mathrm{E}+00$ & $2.41347 \mathrm{E}-10$ & $2.49329 \mathrm{E}-10$ & $2.48393 \mathrm{E}-10$ \\
\hline $\mathrm{Cu}-68 \mathrm{~m}$ & $0.00000 \mathrm{E}+00$ & $0.00000 \mathrm{E}+00$ & $0.00000 \mathrm{E}+00$ & $6.96853 \mathrm{E}-11$ & $7.18471 \mathrm{E}-11$ & $7.15686 \mathrm{E}-11$ \\
\hline Cu-69 & $0.00000 \mathrm{E}+00$ & $0.00000 \mathrm{E}+00$ & $0.00000 \mathrm{E}+00$ & 3.58373E-09 & $3.64838 \mathrm{E}-09$ & 3.62054E-09 \\
\hline $\mathrm{Cu}-70$ & $0.00000 \mathrm{E}+00$ & $0.00000 \mathrm{E}+00$ & $0.00000 \mathrm{E}+00$ & $1.12326 \mathrm{E}-09$ & $1.13240 \mathrm{E}-09$ & $1.12064 \mathrm{E}-09$ \\
\hline $\mathrm{Cu}-70 \mathrm{~m}$ & $0.00000 \mathrm{E}+00$ & $0.00000 \mathrm{E}+00$ & $0.00000 \mathrm{E}+00$ & $6.61620 \mathrm{E}-10$ & $6.65103 \mathrm{E}-10$ & $6.57644 \mathrm{E}-10$ \\
\hline $\mathrm{Cu}-70 \mathrm{~m}$ & $0.00000 \mathrm{E}+00$ & $0.00000 \mathrm{E}+00$ & $0.00000 \mathrm{E}+00$ & $6.61620 \mathrm{E}-10$ & $6.65103 \mathrm{E}-10$ & $6.57644 \mathrm{E}-10$ \\
\hline Cu-71 & $0.00000 \mathrm{E}+00$ & $0.00000 \mathrm{E}+00$ & $0.00000 \mathrm{E}+00$ & 3.75923E-09 & $3.81030 \mathrm{E}-09$ & $3.77568 \mathrm{E}-09$ \\
\hline Cu-72 & $0.00000 \mathrm{E}+00$ & $0.00000 \mathrm{E}+00$ & $0.00000 \mathrm{E}+00$ & $2.24634 \mathrm{E}-09$ & $2.28167 \mathrm{E}-09$ & $2.25738 \mathrm{E}-09$ \\
\hline
\end{tabular}




\section{Depletion Analysis of Modular High Temperature Gas-cooled Reactor Loaded with LEU/Thorium Fuel}

February 2012

\begin{tabular}{|c|c|c|c|c|c|c|}
\hline \multirow[b]{2}{*}{ Nuclide } & \multicolumn{3}{|c|}{ Fresh Fuel } & \multicolumn{3}{|c|}{ Discharge } \\
\hline & Unit Cell & $\begin{array}{l}\text { Full Core } \\
(N O L B P)\end{array}$ & Full Core (3) & Unit Cell & $\begin{array}{l}\text { Full Core } \\
\text { (NO LBP) }\end{array}$ & Full Core (3) \\
\hline $\mathrm{Cu}-73$ & $0.00000 \mathrm{E}+00$ & $0.00000 \mathrm{E}+00$ & $0.00000 \mathrm{E}+00$ & $2.54532 \mathrm{E}-09$ & $2.58801 \mathrm{E}-09$ & $2.56652 \mathrm{E}-09$ \\
\hline $\mathrm{Cu}-74$ & $0.00000 \mathrm{E}+00$ & $0.00000 \mathrm{E}+00$ & $0.00000 \mathrm{E}+00$ & $9.29093 \mathrm{E}-10$ & $9.43502 \mathrm{E}-10$ & $9.37696 \mathrm{E}-10$ \\
\hline $\mathrm{Cu}-75$ & $0.00000 \mathrm{E}+00$ & $0.00000 \mathrm{E}+00$ & $0.00000 \mathrm{E}+00$ & $8.68143 \mathrm{E}-10$ & $8.82836 \mathrm{E}-10$ & $8.79045 \mathrm{E}-10$ \\
\hline $\mathrm{Cu}-76$ & $0.00000 \mathrm{E}+00$ & $0.00000 \mathrm{E}+00$ & $0.00000 \mathrm{E}+00$ & $2.48833 \mathrm{E}-10$ & $2.52617 \mathrm{E}-10$ & $2.52738 \mathrm{E}-10$ \\
\hline $\mathrm{Cu}-77$ & $0.00000 \mathrm{E}+00$ & $0.00000 \mathrm{E}+00$ & $0.00000 \mathrm{E}+00$ & 8.19772E-11 & 8.31487E-11 & $8.34078 \mathrm{E}-11$ \\
\hline $\mathrm{Cu}-78$ & $0.00000 \mathrm{E}+00$ & $0.00000 \mathrm{E}+00$ & $0.00000 \mathrm{E}+00$ & $1.34437 \mathrm{E}-11$ & $1.36530 \mathrm{E}-11$ & $1.37165 \mathrm{E}-11$ \\
\hline $\mathrm{Cu}-79$ & $0.00000 \mathrm{E}+00$ & $0.00000 \mathrm{E}+00$ & $0.00000 \mathrm{E}+00$ & $2.49840 \mathrm{E}-13$ & $2.73402 \mathrm{E}-13$ & $2.68319 \mathrm{E}-13$ \\
\hline $\mathrm{Cu}-80$ & $0.00000 \mathrm{E}+00$ & $0.00000 \mathrm{E}+00$ & $0.00000 \mathrm{E}+00$ & $5.93786 \mathrm{E}-15$ & $6.23032 \mathrm{E}-15$ & $6.22078 \mathrm{E}-15$ \\
\hline$Z n-66$ & $0.00000 \mathrm{E}+00$ & $0.00000 \mathrm{E}+00$ & $0.00000 \mathrm{E}+00$ & $3.94000 \mathrm{E}-05$ & 4.10291E-05 & 24998E-05 \\
\hline$Z n-67$ & $0.00000 \mathrm{E}+00$ & $0.00000 \mathrm{E}+00$ & $0.00000 \mathrm{E}+00$ & $1.44021 \mathrm{E}-04$ & 1.48514E-04 & 51369E-04 \\
\hline$Z n-68$ & $0.00000 \mathrm{E}+00$ & $0.00000 \mathrm{E}+00$ & $0.00000 \mathrm{E}+00$ & $3.43286 \mathrm{E}-04$ & $3.49059 \mathrm{E}-04$ & $.56594 \mathrm{E}-04$ \\
\hline Zn-69 & $0.00000 \mathrm{E}+00$ & $0.00000 \mathrm{E}+00$ & $0.00000 \mathrm{E}+00$ & $7.14852 \mathrm{E}-08$ & $7.27745 \mathrm{E}-08$ & $7.22180 \mathrm{E}-08$ \\
\hline$Z n-69 m$ & $0.00000 \mathrm{E}+00$ & $0.00000 \mathrm{E}+00$ & $0.00000 \mathrm{E}+00$ & $6.72529 \mathrm{E}-09$ & $6.84560 \mathrm{E}-09$ & $6.77637 \mathrm{E}-09$ \\
\hline$Z n-70$ & $0.00000 \mathrm{E}+00$ & $0.00000 \mathrm{E}+00$ & $0.00000 \mathrm{E}+00$ & $2.74474 \mathrm{E}-03$ & $2.74092 \mathrm{E}-03$ & $2.81984 \mathrm{E}-03$ \\
\hline$Z n-71$ & $0.00000 \mathrm{E}+00$ & $0.00000 \mathrm{E}+00$ & $0.00000 \mathrm{E}+00$ & $2.92181 \mathrm{E}-08$ & $2.96118 \mathrm{E}-08$ & $2.93409 \mathrm{E}-08$ \\
\hline$Z n-71 m$ & $0.00000 \mathrm{E}+00$ & $0.00000 \mathrm{E}+00$ & $0.00000 \mathrm{E}+00$ & $3.65329 \mathrm{E}-07$ & $3.69014 \mathrm{E}-07$ & $3.64878 \mathrm{E}-07$ \\
\hline$Z n-72$ & $0.00000 \mathrm{E}+00$ & $0.00000 \mathrm{E}+00$ & $0.00000 \mathrm{E}+00$ & $9.55172 \mathrm{E}-05$ & $9.69108 \mathrm{E}-05$ & $9.57894 \mathrm{E}-05$ \\
\hline$Z n-73$ & $0.00000 \mathrm{E}+00$ & $0.00000 \mathrm{E}+00$ & $0.00000 \mathrm{E}+00$ & $3.57630 \mathrm{E}-08$ & 3.63174E-08 & $3.59527 \mathrm{E}-08$ \\
\hline$Z n-74$ & $0.00000 \mathrm{E}+00$ & $0.00000 \mathrm{E}+00$ & $0.00000 \mathrm{E}+00$ & $3.44846 \mathrm{E}-07$ & 3.49969E-07 & $3.46960 \mathrm{E}-07$ \\
\hline$Z n-75$ & $0.00000 \mathrm{E}+00$ & $0.00000 \mathrm{E}+00$ & $0.00000 \mathrm{E}+00$ & $8.31186 \mathrm{E}-08$ & $8.44836 \mathrm{E}-08$ & $8.39055 \mathrm{E}-08$ \\
\hline$Z n-76$ & $0.00000 \mathrm{E}+00$ & $0.00000 \mathrm{E}+00$ & $0.00000 \mathrm{E}+00$ & $7.21244 \mathrm{E}-08$ & $7.32573 \mathrm{E}-08$ & $7.29910 \mathrm{E}-08$ \\
\hline$Z n-77$ & $0.00000 \mathrm{E}+00$ & $0.00000 \mathrm{E}+00$ & $0.00000 \mathrm{E}+00$ & $2.90905 \mathrm{E}-08$ & $2.94328 \mathrm{E}-08$ & $2.94757 \mathrm{E}-08$ \\
\hline$Z n-78$ & $0.00000 \mathrm{E}+00$ & $0.00000 \mathrm{E}+00$ & $0.00000 \mathrm{E}+00$ & $1.94877 \mathrm{E}-08$ & $1.96657 \mathrm{E}-08$ & $1.97578 \mathrm{E}-08$ \\
\hline$Z n-79$ & $0.00000 \mathrm{E}+00$ & $0.00000 \mathrm{E}+00$ & $0.00000 \mathrm{E}+00$ & $5.49409 \mathrm{E}-09$ & 5.54256E-09 & 5.57857E-09 \\
\hline$Z n-80$ & $0.00000 \mathrm{E}+00$ & $0.00000 \mathrm{E}+00$ & $0.00000 \mathrm{E}+00$ & $4.75932 \mathrm{E}-10$ & $4.81825 \mathrm{E}-10$ & $4.84177 \mathrm{E}-10$ \\
\hline$Z n-81$ & $0.00000 \mathrm{E}+00$ & $0.00000 \mathrm{E}+00$ & $0.00000 \mathrm{E}+00$ & $8.07718 \mathrm{E}-12$ & $8.62161 \mathrm{E}-12$ & $8.47804 \mathrm{E}-12$ \\
\hline$Z n-82$ & $0.00000 \mathrm{E}+00$ & $0.00000 \mathrm{E}+00$ & $0.00000 \mathrm{E}+00$ & $2.21145 \mathrm{E}-12$ & $2.24292 \mathrm{E}-12$ & $2.26020 \mathrm{E}-12$ \\
\hline$Z n-83$ & $0.00000 \mathrm{E}+00$ & $0.00000 \mathrm{E}+00$ & $0.00000 \mathrm{E}+00$ & $1.56236 \mathrm{E}-14$ & $1.70848 \mathrm{E}-14$ & $1.69428 \mathrm{E}-14$ \\
\hline$G a-66$ & $0.00000 \mathrm{E}+00$ & $0.00000 \mathrm{E}+00$ & $0.00000 \mathrm{E}+00$ & $2.23168 \mathrm{E}-17$ & $2.81930 \mathrm{E}-17$ & $2.93867 \mathrm{E}-17$ \\
\hline$G a-67$ & $0.00000 \mathrm{E}+00$ & $0.00000 \mathrm{E}+00$ & $0.00000 \mathrm{E}+00$ & $1.14144 \mathrm{E}-14$ & $1.44114 \mathrm{E}-14$ & $1.50312 \mathrm{E}-14$ \\
\hline Ga-68 & $0.00000 \mathrm{E}+00$ & $0.00000 \mathrm{E}+00$ & $0.00000 \mathrm{E}+00$ & $2.62528 \mathrm{E}-14$ & $2.96284 \mathrm{E}-14$ & $3.01105 \mathrm{E}-14$ \\
\hline$G a-70$ & $0.00000 \mathrm{E}+00$ & $0.00000 \mathrm{E}+00$ & $0.00000 \mathrm{E}+00$ & $3.65188 \mathrm{E}-10$ & $3.85293 \mathrm{E}-10$ & 3.79894E-10 \\
\hline$G a-72$ & $0.00000 \mathrm{E}+00$ & $0.00000 \mathrm{E}+00$ & $0.00000 \mathrm{E}+00$ & $2.93333 \mathrm{E}-05$ & $2.97677 \mathrm{E}-05$ & $2.94249 \mathrm{E}-05$ \\
\hline$G a-73$ & $0.00000 \mathrm{E}+00$ & $0.00000 \mathrm{E}+00$ & $0.00000 \mathrm{E}+00$ & $2.77965 \mathrm{E}-05$ & $2.82186 \mathrm{E}-05$ & $2.79301 \mathrm{E}-05$ \\
\hline$G a-74$ & $0.00000 \mathrm{E}+00$ & $0.00000 \mathrm{E}+00$ & $0.00000 \mathrm{E}+00$ & $1.91965 \mathrm{E}-06$ & $1.94712 \mathrm{E}-06$ & $1.92953 \mathrm{E}-06$ \\
\hline$G a-74 m$ & $0.00000 \mathrm{E}+00$ & $0.00000 \mathrm{E}+00$ & $0.00000 \mathrm{E}+00$ & $1.97782 \mathrm{E}-09$ & $1.99432 \mathrm{E}-09$ & $1.96692 \mathrm{E}-09$ \\
\hline
\end{tabular}




\begin{tabular}{|c|c|c|c|c|c|c|}
\hline \multirow[b]{2}{*}{ Nuclide } & \multicolumn{3}{|c|}{ Fresh Fuel } & \multicolumn{3}{|c|}{ Discharge } \\
\hline & Unit Cell & $\begin{array}{l}\text { Full Core } \\
\text { (NO LBP) }\end{array}$ & Full Core (3) & Unit Cell & $\begin{array}{l}\text { Full Core } \\
\text { (NO LBP) }\end{array}$ & Full Core (3) \\
\hline$G a-75$ & $0.00000 \mathrm{E}+00$ & $0.00000 \mathrm{E}+00$ & $0.00000 \mathrm{E}+00$ & $1.43316 \mathrm{E}-06$ & $1.45541 \mathrm{E}-06$ & $1.44383 \mathrm{E}-06$ \\
\hline Ga-76 & $0.00000 \mathrm{E}+00$ & $0.00000 \mathrm{E}+00$ & $0.00000 \mathrm{E}+00$ & $7.32692 \mathrm{E}-07$ & $7.42826 \mathrm{E}-07$ & $7.38933 \mathrm{E}-07$ \\
\hline Ga-77 & $0.00000 \mathrm{E}+00$ & $0.00000 \mathrm{E}+00$ & $0.00000 \mathrm{E}+00$ & $5.65550 \mathrm{E}-07$ & $5.71930 \mathrm{E}-07$ & $5.70319 \mathrm{E}-07$ \\
\hline Ga-78 & $0.00000 \mathrm{E}+00$ & $0.00000 \mathrm{E}+00$ & $0.00000 \mathrm{E}+00$ & $3.39352 \mathrm{E}-07$ & $3.41915 \mathrm{E}-07$ & 3.42103E-07 \\
\hline Ga-79 & $0.00000 \mathrm{E}+00$ & $0.00000 \mathrm{E}+00$ & $0.00000 \mathrm{E}+00$ & $2.26201 \mathrm{E}-07$ & $2.28007 \mathrm{E}-07$ & $2.28527 \mathrm{E}-07$ \\
\hline$G a-80$ & $0.00000 \mathrm{E}+00$ & $0.00000 \mathrm{E}+00$ & $0.00000 \mathrm{E}+00$ & $7.89565 \mathrm{E}-08$ & $7.94623 \mathrm{E}-08$ & $7.97471 \mathrm{E}-08$ \\
\hline$G a-81$ & $0.00000 \mathrm{E}+00$ & $0.00000 \mathrm{E}+00$ & $0.00000 \mathrm{E}+00$ & $3.63611 \mathrm{E}-08$ & $3.66264 \mathrm{E}-08$ & $3.68213 \mathrm{E}-08$ \\
\hline Ga-82 & $0.00000 \mathrm{E}+00$ & $0.00000 \mathrm{E}+00$ & $0.00000 \mathrm{E}+00$ & $1.18439 \mathrm{E}-08$ & $1.19223 \mathrm{E}-08$ & $1.20277 \mathrm{E}-08$ \\
\hline Ga-83 & $0.00000 \mathrm{E}+00$ & $0.00000 \mathrm{E}+00$ & $0.00000 \mathrm{E}+00$ & $2.38690 \mathrm{E}-10$ & $2.45121 \mathrm{E}-10$ & $2.45805 \mathrm{E}-10$ \\
\hline$G a-84$ & $0.00000 \mathrm{E}+00$ & $0.00000 \mathrm{E}+00$ & $0.00000 \mathrm{E}+00$ & $2.76126 \mathrm{E}-09$ & $2.77043 \mathrm{E}-09$ & $2.80133 \mathrm{E}-09$ \\
\hline$G a-85$ & $0.00000 \mathrm{E}+00$ & $0.00000 \mathrm{E}+00$ & $0.00000 \mathrm{E}+00$ & $1.77495 \mathrm{E}-13$ & $1.94482 \mathrm{E}-13$ & $1.92743 \mathrm{E}-13$ \\
\hline$G a-86$ & $0.00000 \mathrm{E}+00$ & $0.00000 \mathrm{E}+00$ & $0.00000 \mathrm{E}+00$ & $2.85883 \mathrm{E}-12$ & $2.86857 \mathrm{E}-12$ & $2.90054 \mathrm{E}-12$ \\
\hline$G e-66$ & $0.00000 \mathrm{E}+00$ & $0.00000 \mathrm{E}+00$ & $0.00000 \mathrm{E}+00$ & $0.00000 \mathrm{E}+00$ & $0.00000 \mathrm{E}+00$ & $0.00000 \mathrm{E}+00$ \\
\hline$G e-67$ & $0.00000 \mathrm{E}+00$ & $0.00000 \mathrm{E}+00$ & $0.00000 \mathrm{E}+00$ & $0.00000 \mathrm{E}+00$ & $0.00000 \mathrm{E}+00$ & $0.00000 \mathrm{E}+00$ \\
\hline$G e-68$ & $0.00000 \mathrm{E}+00$ & $0.00000 \mathrm{E}+00$ & $0.00000 \mathrm{E}+00$ & $1.95246 \mathrm{E}-15$ & $2.44744 \mathrm{E}-15$ & $2.64523 \mathrm{E}-15$ \\
\hline Ge-69 & $0.00000 \mathrm{E}+00$ & $0.00000 \mathrm{E}+00$ & $0.00000 \mathrm{E}+00$ & $3.04442 \mathrm{E}-15$ & $3.80330 \mathrm{E}-15$ & $3.93859 \mathrm{E}-15$ \\
\hline$G e-71$ & $0.00000 \mathrm{E}+00$ & $0.00000 \mathrm{E}+00$ & $0.00000 \mathrm{E}+00$ & $1.54333 \mathrm{E}-09$ & $1.63895 \mathrm{E}-09$ & $1.62493 \mathrm{E}-09$ \\
\hline Ge-71m & $0.00000 \mathrm{E}+00$ & $0.00000 \mathrm{E}+00$ & $0.00000 \mathrm{E}+00$ & 3.93709E-18 & $3.98754 \mathrm{E}-18$ & $3.93724 \mathrm{E}-18$ \\
\hline$G e-73 m$ & $0.00000 \mathrm{E}+00$ & $0.00000 \mathrm{E}+00$ & $0.00000 \mathrm{E}+00$ & $5.55559 \mathrm{E}-11$ & $5.63991 \mathrm{E}-11$ & $5.58220 \mathrm{E}-11$ \\
\hline$G e-75$ & $0.00000 \mathrm{E}+00$ & $0.00000 \mathrm{E}+00$ & $0.00000 \mathrm{E}+00$ & $5.76644 \mathrm{E}-05$ & 5.85554E-05 & $5.80822 \mathrm{E}-05$ \\
\hline$G e-75 m$ & $0.00000 \mathrm{E}+00$ & $0.00000 \mathrm{E}+00$ & $0.00000 \mathrm{E}+00$ & $9.55496 \mathrm{E}-09$ & $9.66119 \mathrm{E}-09$ & $9.52463 \mathrm{E}-09$ \\
\hline Ge-77 & $0.00000 \mathrm{E}+00$ & $0.00000 \mathrm{E}+00$ & $0.00000 \mathrm{E}+00$ & $2.18275 \mathrm{E}-03$ & $2.20709 \mathrm{E}-03$ & $2.19707 \mathrm{E}-03$ \\
\hline Ge-77m & $0.00000 \mathrm{E}+00$ & $0.00000 \mathrm{E}+00$ & $0.00000 \mathrm{E}+00$ & $8.26404 \mathrm{E}-08$ & $8.34869 \mathrm{E}-08$ & $.25349 \mathrm{E}-08$ \\
\hline $\mathrm{Ge}-78$ & $0.00000 \mathrm{E}+00$ & $0.00000 \mathrm{E}+00$ & $0.00000 \mathrm{E}+00$ & 7.04487E-04 & 7.09961E-04 & 7.07596E-04 \\
\hline$G e-79$ & $0.00000 \mathrm{E}+00$ & $0.00000 \mathrm{E}+00$ & $0.00000 \mathrm{E}+00$ & $3.60259 \mathrm{E}-06$ & $3.63688 \mathrm{E}-06$ & $3.62601 \mathrm{E}-06$ \\
\hline$G e-79 m$ & $0.00000 \mathrm{E}+00$ & $0.00000 \mathrm{E}+00$ & $0.00000 \mathrm{E}+00$ & 4.13869E-06 & 4.18264E-06 & 4.15432E-06 \\
\hline Ge- 80 & $0.00000 \mathrm{E}+00$ & $0.00000 \mathrm{E}+00$ & $0.00000 \mathrm{E}+00$ & $1.71670 \mathrm{E}-05$ & $1.72987 \mathrm{E}-05$ & $1.72879 \mathrm{E}-05$ \\
\hline Ge- 81 & $0.00000 \mathrm{E}+00$ & $0.00000 \mathrm{E}+00$ & $0.00000 \mathrm{E}+00$ & 4.58944E-06 & $4.61641 \mathrm{E}-06$ & 4.62364E-06 \\
\hline$G e-82$ & $0.00000 \mathrm{E}+00$ & $0.00000 \mathrm{E}+00$ & $0.00000 \mathrm{E}+00$ & $2.35020 \mathrm{E}-06$ & $2.36520 \mathrm{E}-06$ & $2.37632 \mathrm{E}-06$ \\
\hline$G e-83$ & $0.00000 \mathrm{E}+00$ & $0.00000 \mathrm{E}+00$ & $0.00000 \mathrm{E}+00$ & $3.42702 \mathrm{E}-07$ & $3.45600 \mathrm{E}-07$ & $3.47386 \mathrm{E}-07$ \\
\hline$G e-84$ & $0.00000 \mathrm{E}+00$ & $0.00000 \mathrm{E}+00$ & $0.00000 \mathrm{E}+00$ & $1.05957 \mathrm{E}-07$ & $1.07027 \mathrm{E}-07$ & $1.07665 \mathrm{E}-07$ \\
\hline$G e-85$ & $0.00000 \mathrm{E}+00$ & $0.00000 \mathrm{E}+00$ & $0.00000 \mathrm{E}+00$ & 4.65251E-09 & 4.75167E-09 & 4.77005E-09 \\
\hline Ge- 86 & $0.00000 \mathrm{E}+00$ & $0.00000 \mathrm{E}+00$ & $0.00000 \mathrm{E}+00$ & $1.60467 \mathrm{E}-07$ & $1.60986 \mathrm{E}-07$ & $1.62785 \mathrm{E}-07$ \\
\hline$G e-87$ & $0.00000 \mathrm{E}+00$ & $0.00000 \mathrm{E}+00$ & $0.00000 \mathrm{E}+00$ & $9.35688 \mathrm{E}-10$ & $9.38933 \mathrm{E}-10$ & $9.49372 \mathrm{E}-10$ \\
\hline$G e-88$ & $0.00000 \mathrm{E}+00$ & $0.00000 \mathrm{E}+00$ & $0.00000 \mathrm{E}+00$ & $1.05998 \mathrm{E}-11$ & $1.06437 \mathrm{E}-11$ & $1.07605 \mathrm{E}-11$ \\
\hline$G e-89$ & $0.00000 \mathrm{E}+00$ & $0.00000 \mathrm{E}+00$ & $0.00000 \mathrm{E}+00$ & $2.73535 \mathrm{E}-15$ & $3.25289 \mathrm{E}-15$ & $3.17857 \mathrm{E}-15$ \\
\hline
\end{tabular}




\section{Depletion Analysis of Modular High Temperature Gas-cooled Reactor Loaded with LEU/Thorium Fuel}

February 2012

\begin{tabular}{|c|c|c|c|c|c|c|}
\hline \multirow[b]{2}{*}{ Nuclide } & \multicolumn{3}{|c|}{ Fresh Fuel } & \multicolumn{3}{|c|}{ Discharge } \\
\hline & Unit Cell & $\begin{array}{l}\text { Full Core } \\
(N O L B P)\end{array}$ & Full Core (3) & Unit Cell & $\begin{array}{l}\text { Full Core } \\
(N O L B P)\end{array}$ & Full Core (3) \\
\hline$A s-69$ & $0.00000 \mathrm{E}+00$ & $0.00000 \mathrm{E}+00$ & $0.00000 \mathrm{E}+00$ & $0.00000 \mathrm{E}+00$ & $0.00000 \mathrm{E}+00$ & $0.00000 \mathrm{E}+00$ \\
\hline$A s-71$ & $0.00000 \mathrm{E}+00$ & $0.00000 \mathrm{E}+00$ & $0.00000 \mathrm{E}+00$ & $6.68607 \mathrm{E}-16$ & $8.44633 \mathrm{E}-16$ & $8.80821 \mathrm{E}-16$ \\
\hline$A s-72$ & $0.00000 \mathrm{E}+00$ & $0.00000 \mathrm{E}+00$ & $0.00000 \mathrm{E}+00$ & $1.99421 \mathrm{E}-13$ & $2.00181 \mathrm{E}-13$ & $1.96852 \mathrm{E}-13$ \\
\hline$A s-73$ & $0.00000 \mathrm{E}+00$ & $0.00000 \mathrm{E}+00$ & $0.00000 \mathrm{E}+00$ & $1.51180 \mathrm{E}-09$ & $1.50236 \mathrm{E}-09$ & $1.49512 \mathrm{E}-09$ \\
\hline$A s-75 m$ & $0.00000 \mathrm{E}+00$ & $0.00000 \mathrm{E}+00$ & $0.00000 \mathrm{E}+00$ & $5.24353 \mathrm{E}-19$ & 5.45696E-19 & $5.59013 \mathrm{E}-19$ \\
\hline$A s-76$ & $0.00000 \mathrm{E}+00$ & $0.00000 \mathrm{E}+00$ & $0.00000 \mathrm{E}+00$ & 4.42723E-05 & 4.66029E-05 & $4.54181 \mathrm{E}-05$ \\
\hline$A s-77$ & $0.00000 \mathrm{E}+00$ & $0.00000 \mathrm{E}+00$ & $0.00000 \mathrm{E}+00$ & $7.72415 \mathrm{E}-03$ & $7.80990 \mathrm{E}-03$ & $7.77340 \mathrm{E}-03$ \\
\hline$A s-78$ & $0.00000 \mathrm{E}+00$ & $0.00000 \mathrm{E}+00$ & $0.00000 \mathrm{E}+00$ & $7.41532 \mathrm{E}-04$ & 7.47172E-04 & $7.44535 \mathrm{E}-04$ \\
\hline$A s-79$ & $0.00000 \mathrm{E}+00$ & $0.00000 \mathrm{E}+00$ & $0.00000 \mathrm{E}+00$ & $1.72376 \mathrm{E}-04$ & $1.73953 \mathrm{E}-04$ & $1.73125 \mathrm{E}-04$ \\
\hline$A s-80$ & $0.00000 \mathrm{E}+00$ & $0.00000 \mathrm{E}+00$ & $0.00000 \mathrm{E}+00$ & $1.07115 \mathrm{E}-05$ & $1.07828 \mathrm{E}-05$ & $07621 \mathrm{E}-05$ \\
\hline$A s-81$ & $0.00000 \mathrm{E}+00$ & $0.00000 \mathrm{E}+00$ & $0.00000 \mathrm{E}+00$ & $3.72805 \mathrm{E}-05$ & $3.74869 \mathrm{E}-05$ & 74014E-05 \\
\hline$A s-82$ & $0.00000 \mathrm{E}+00$ & $0.00000 \mathrm{E}+00$ & $0.00000 \mathrm{E}+00$ & $2.16747 \mathrm{E}-05$ & $2.18016 \mathrm{E}-05$ & $8608 \mathrm{E}-05$ \\
\hline$A s-82 m$ & $0.00000 \mathrm{E}+00$ & $0.00000 \mathrm{E}+00$ & $0.00000 \mathrm{E}+00$ & 4.45303E-06 & 4.48587E-06 & 4.45041E-06 \\
\hline$A s-83$ & $0.00000 \mathrm{E}+00$ & $0.00000 \mathrm{E}+00$ & $0.00000 \mathrm{E}+00$ & & $2.27616 \mathrm{E}-05$ & $2.27829 \mathrm{E}-05$ \\
\hline$A s-84$ & $0.00000 \mathrm{E}+00$ & $0.00000 \mathrm{E}+00$ & $0.00000 \mathrm{E}+00$ & $1.79770 \mathrm{E}-06$ & $1.80976 \mathrm{E}-06$ & $1.81632 \mathrm{E}-06$ \\
\hline$A s-85$ & $0.00000 \mathrm{E}+00$ & $0.00000 \mathrm{E}+00$ & $0.00000 \mathrm{E}+00$ & $1.06762 \mathrm{E}-06$ & $1.07901 \mathrm{E}-06$ & $1.08351 \mathrm{E}-06$ \\
\hline$A s-86$ & $0.00000 \mathrm{E}+00$ & $0.00000 \mathrm{E}+00$ & $0.00000 \mathrm{E}+00$ & $1.90154 \mathrm{E}-06$ & $1.90934 \mathrm{E}-06$ & $1.92921 \mathrm{E}-06$ \\
\hline As- 87 & $0.00000 \mathrm{E}+00$ & $0.00000 \mathrm{E}+00$ & $0.00000 \mathrm{E}+00$ & $9.42824 \mathrm{E}-08$ & 9.47594E-08 & $9.57032 \mathrm{E}-08$ \\
\hline$A s-88$ & $0.00000 \mathrm{E}+00$ & $0.00000 \mathrm{E}+00$ & $0.00000 \mathrm{E}+00$ & $4.28880 \mathrm{E}-08$ & 4.30337E-08 & $4.35121 \mathrm{E}-08$ \\
\hline$A s-89$ & $0.00000 \mathrm{E}+00$ & $0.00000 \mathrm{E}+00$ & $0.00000 \mathrm{E}+00$ & $3.39331 \mathrm{E}-11$ & 3.47914E-11 & 3.49823E-11 \\
\hline$A s-90$ & $0.00000 \mathrm{E}+00$ & $0.00000 \mathrm{E}+00$ & $0.00000 \mathrm{E}+00$ & $8.36533 \mathrm{E}-13$ & $8.48740 \mathrm{E}-13$ & $8.50332 \mathrm{E}-13$ \\
\hline$A s-91$ & $0.00000 \mathrm{E}+00$ & $0.00000 \mathrm{E}+00$ & $0.00000 \mathrm{E}+00$ & $4.76905 \mathrm{E}-14$ & $5.01940 \mathrm{E}-14$ & $5.02211 \mathrm{E}-14$ \\
\hline$A s-92$ & $0.00000 \mathrm{E}+00$ & $0.00000 \mathrm{E}+00$ & $0.00000 \mathrm{E}+00$ & $6.54144 \mathrm{E}-16$ & 7.99116E-16 & $7.77032 \mathrm{E}-16$ \\
\hline $\mathrm{Se}-72$ & $0.00000 \mathrm{E}+00$ & $0.00000 \mathrm{E}+00$ & $0.00000 \mathrm{E}+00$ & $0.00000 \mathrm{E}+00$ & $0.00000 \mathrm{E}+00$ & $0.00000 \mathrm{E}+00$ \\
\hline $\mathrm{Se}-73$ & $0.00000 \mathrm{E}+00$ & $0.00000 \mathrm{E}+00$ & $0.00000 \mathrm{E}+00$ & $2.35679 \mathrm{E}-17$ & $2.97378 \mathrm{E}-17$ & 3.09917E-17 \\
\hline $\mathrm{Se}-73 \mathrm{~m}$ & $0.00000 \mathrm{E}+00$ & $0.00000 \mathrm{E}+00$ & $0.00000 \mathrm{E}+00$ & $4.26675 \mathrm{E}-19$ & 5.39024E-19 & $5.61801 \mathrm{E}-19$ \\
\hline $\mathrm{Se}-75$ & $0.00000 \mathrm{E}+00$ & $0.00000 \mathrm{E}+00$ & $0.00000 \mathrm{E}+00$ & 4.75503E-09 & 4.94858E-09 & $5.06934 \mathrm{E}-09$ \\
\hline$S e-77 m$ & $0.00000 \mathrm{E}+00$ & $0.00000 \mathrm{E}+00$ & $0.00000 \mathrm{E}+00$ & $3.19941 \mathrm{E}-09$ & $3.23480 \mathrm{E}-09$ & 3.21957E-09 \\
\hline $\mathrm{Se}-79 \mathrm{~m}$ & $0.00000 \mathrm{E}+00$ & $0.00000 \mathrm{E}+00$ & $0.00000 \mathrm{E}+00$ & 7.05127E-05 & $7.11581 \mathrm{E}-05$ & $7.08190 \mathrm{E}-05$ \\
\hline Se-81 & $0.00000 \mathrm{E}+00$ & $0.00000 \mathrm{E}+00$ & $0.00000 \mathrm{E}+00$ & $1.32921 \mathrm{E}-03$ & $1.33616 \mathrm{E}-03$ & $1.33237 \mathrm{E}-03$ \\
\hline $\mathrm{Se}-81 \mathrm{~m}$ & $0.00000 \mathrm{E}+00$ & $0.00000 \mathrm{E}+00$ & $0.00000 \mathrm{E}+00$ & $2.62473 \mathrm{E}-04$ & $2.62581 \mathrm{E}-04$ & 2.60205E-04 \\
\hline $\mathrm{Se}-83$ & $0.00000 \mathrm{E}+00$ & $0.00000 \mathrm{E}+00$ & $0.00000 \mathrm{E}+00$ & $3.59332 \mathrm{E}-03$ & $3.62188 \mathrm{E}-03$ & 3.61912E-03 \\
\hline$S e-83 m$ & $0.00000 \mathrm{E}+00$ & $0.00000 \mathrm{E}+00$ & $0.00000 \mathrm{E}+00$ & $1.72870 \mathrm{E}-05$ & $1.73973 \mathrm{E}-05$ & $1.73174 \mathrm{E}-05$ \\
\hline $\mathrm{Se}-84$ & $0.00000 \mathrm{E}+00$ & $0.00000 \mathrm{E}+00$ & $0.00000 \mathrm{E}+00$ & $7.77098 \mathrm{E}-04$ & 7.83096E-04 & $7.82741 \mathrm{E}-04$ \\
\hline $\mathrm{Se}-85$ & $0.00000 \mathrm{E}+00$ & $0.00000 \mathrm{E}+00$ & $0.00000 \mathrm{E}+00$ & $8.90545 \mathrm{E}-05$ & $8.96968 \mathrm{E}-05$ & $8.98137 \mathrm{E}-05$ \\
\hline $\mathrm{Se}-86$ & $0.00000 \mathrm{E}+00$ & $0.00000 \mathrm{E}+00$ & $0.00000 \mathrm{E}+00$ & $9.07466 \mathrm{E}-05$ & $9.12543 \mathrm{E}-05$ & $9.17173 \mathrm{E}-05$ \\
\hline
\end{tabular}




\begin{tabular}{|c|c|c|c|c|c|c|}
\hline \multirow[b]{2}{*}{ Nuclide } & \multicolumn{3}{|c|}{ Fresh Fuel } & \multicolumn{3}{|c|}{ Discharge } \\
\hline & Unit Cell & $\begin{array}{l}\text { Full Core } \\
\text { (NO LBP) }\end{array}$ & Full Core (3) & Unit Cell & $\begin{array}{l}\text { Full Core } \\
(N O L B P)\end{array}$ & Full Core (3) \\
\hline $\mathrm{Se}-87$ & $0.00000 \mathrm{E}+00$ & $0.00000 \mathrm{E}+00$ & $0.00000 \mathrm{E}+00$ & $1.67854 \mathrm{E}-05$ & $1.68757 \mathrm{E}-05$ & $1.69756 \mathrm{E}-05$ \\
\hline $\mathrm{Se}-88$ & $0.00000 \mathrm{E}+00$ & $0.00000 \mathrm{E}+00$ & $0.00000 \mathrm{E}+00$ & 2.19894E-06 & $2.21235 \mathrm{E}-06$ & $2.22857 \mathrm{E}-06$ \\
\hline $\mathrm{Se}-89$ & $0.00000 \mathrm{E}+00$ & $0.00000 \mathrm{E}+00$ & $0.00000 \mathrm{E}+00$ & $8.02539 \mathrm{E}-08$ & $8.11890 \mathrm{E}-08$ & $8.16746 \mathrm{E}-08$ \\
\hline $\mathrm{Se}-90$ & $0.00000 \mathrm{E}+00$ & $0.00000 \mathrm{E}+00$ & $0.00000 \mathrm{E}+00$ & $8.93549 \mathrm{E}-09$ & $9.02356 \mathrm{E}-09$ & 07620E-09 \\
\hline $\mathrm{Se}-91$ & $0.00000 \mathrm{E}+00$ & $0.00000 \mathrm{E}+00$ & $0.00000 \mathrm{E}+00$ & $6.95724 \mathrm{E}-10$ & $7.15334 \mathrm{E}-10$ & $7.19340 \mathrm{E}-10$ \\
\hline $\mathrm{Se}-92$ & $0.00000 \mathrm{E}+00$ & $0.00000 \mathrm{E}+00$ & $0.00000 \mathrm{E}+00$ & $1.73829 \mathrm{E}-11$ & $1.80587 \mathrm{E}-11$ & $1.80925 \mathrm{E}-11$ \\
\hline $\mathrm{Se}-93$ & $0.00000 \mathrm{E}+00$ & $0.00000 \mathrm{E}+00$ & $0.00000 \mathrm{E}+00$ & $1.61730 \mathrm{E}-12$ & $1.65586 \mathrm{E}-12$ & $1.65028 \mathrm{E}-12$ \\
\hline $\mathrm{Se}-94$ & $0.00000 \mathrm{E}+00$ & $0.00000 \mathrm{E}+00$ & $0.00000 \mathrm{E}+00$ & $2.96722 \mathrm{E}-14$ & $3.04750 \mathrm{E}-14$ & $2.98370 \mathrm{E}-14$ \\
\hline$B r-75$ & $0.00000 \mathrm{E}+00$ & $0.00000 \mathrm{E}+00$ & $0.00000 \mathrm{E}+00$ & $9.04388 \mathrm{E}-19$ & $1.14252 \mathrm{E}-18$ & $1.19082 \mathrm{E}-18$ \\
\hline$B r-76$ & $0.00000 \mathrm{E}+00$ & $0.00000 \mathrm{E}+00$ & $0.00000 \mathrm{E}+00$ & $1.57828 \mathrm{E}-27$ & $1.99695 \mathrm{E}-27$ & $2.12348 \mathrm{E}-27$ \\
\hline$B r-77$ & $0.00000 \mathrm{E}+00$ & $0.00000 \mathrm{E}+00$ & $0.00000 \mathrm{E}+00$ & $1.02714 \mathrm{E}-11$ & $1.02083 \mathrm{E}-11$ & $1.00084 \mathrm{E}-11$ \\
\hline$B r-77 m$ & $0.00000 \mathrm{E}+00$ & $0.00000 \mathrm{E}+00$ & $0.00000 \mathrm{E}+00$ & $9.88874 \mathrm{E}-15$ & $9.82974 \mathrm{E}-15$ & $9.63458 \mathrm{E}-15$ \\
\hline$B r-78$ & $0.00000 \mathrm{E}+00$ & $0.00000 \mathrm{E}+00$ & $0.00000 \mathrm{E}+00$ & $2.08478 \mathrm{E}-12$ & $2.06346 \mathrm{E}-12$ & $2.02266 \mathrm{E}-12$ \\
\hline$B r-79 m$ & $0.00000 \mathrm{E}+00$ & $0.00000 \mathrm{E}+00$ & $0.00000 \mathrm{E}+00$ & $1.68576 \mathrm{E}-12$ & $1.67422 \mathrm{E}-12$ & $1.64105 \mathrm{E}-12$ \\
\hline$B r-80$ & $0.00000 \mathrm{E}+00$ & $0.00000 \mathrm{E}+00$ & $0.00000 \mathrm{E}+00$ & $3.04233 \mathrm{E}-08$ & $3.14790 \mathrm{E}-08$ & $3.07346 \mathrm{E}-08$ \\
\hline$B r-80 m$ & $0.00000 \mathrm{E}+00$ & $0.00000 \mathrm{E}+00$ & $0.00000 \mathrm{E}+00$ & $1.38078 \mathrm{E}-07$ & $1.40623 \mathrm{E}-07$ & $1.38832 \mathrm{E}-07$ \\
\hline$B r-82$ & $0.00000 \mathrm{E}+00$ & $0.00000 \mathrm{E}+00$ & $0.00000 \mathrm{E}+00$ & $6.20344 \mathrm{E}-03$ & $6.54241 \mathrm{E}-03$ & $6.30414 \mathrm{E}-03$ \\
\hline$B r-82 m$ & $0.00000 \mathrm{E}+00$ & $0.00000 \mathrm{E}+00$ & $0.00000 \mathrm{E}+00$ & $3.13949 \mathrm{E}-07$ & $3.12109 \mathrm{E}-07$ & $3.06948 \mathrm{E}-07$ \\
\hline$B r-83$ & $0.00000 \mathrm{E}+00$ & $0.00000 \mathrm{E}+00$ & $0.00000 \mathrm{E}+00$ & $2.63430 \mathrm{E}-02$ & $2.65464 \mathrm{E}-02$ & $2.65159 \mathrm{E}-02$ \\
\hline$B r-84$ & $0.00000 \mathrm{E}+00$ & $0.00000 \mathrm{E}+00$ & $0.00000 \mathrm{E}+00$ & $8.30386 \mathrm{E}-03$ & $8.36796 \mathrm{E}-03$ & $8.36205 \mathrm{E}-03$ \\
\hline$B r-84 m$ & $0.00000 \mathrm{E}+00$ & $0.00000 \mathrm{E}+00$ & $0.00000 \mathrm{E}+00$ & $9.43301 \mathrm{E}-05$ & 9.51544E-05 & $9.41986 \mathrm{E}-05$ \\
\hline$B r-85$ & $0.00000 \mathrm{E}+00$ & $0.00000 \mathrm{E}+00$ & $0.00000 \mathrm{E}+00$ & 8.06494E-04 & $8.13629 \mathrm{E}-04$ & $8.12978 \mathrm{E}-04$ \\
\hline$B r-86$ & $0.00000 \mathrm{E}+00$ & $0.00000 \mathrm{E}+00$ & $0.00000 \mathrm{E}+00$ & $4.21258 \mathrm{E}-04$ & 4.23866E-04 & 4.25177E-04 \\
\hline$B r-87$ & $0.00000 \mathrm{E}+00$ & $0.00000 \mathrm{E}+00$ & $0.00000 \mathrm{E}+00$ & 5.40761E-04 & $5.44068 \mathrm{E}-04$ & 5.45357E-04 \\
\hline$B r-88$ & $0.00000 \mathrm{E}+00$ & $0.00000 \mathrm{E}+00$ & $0.00000 \mathrm{E}+00$ & $1.28522 \mathrm{E}-04$ & $1.29200 \mathrm{E}-04$ & $1.29685 \mathrm{E}-04$ \\
\hline$B r-89$ & $0.00000 \mathrm{E}+00$ & $0.00000 \mathrm{E}+00$ & $0.00000 \mathrm{E}+00$ & $2.12489 \mathrm{E}-05$ & $2.13495 \mathrm{E}-05$ & $2.14335 \mathrm{E}-05$ \\
\hline Br-90 & $0.00000 \mathrm{E}+00$ & $0.00000 \mathrm{E}+00$ & $0.00000 \mathrm{E}+00$ & 4.81103E-06 & $4.82729 \mathrm{E}-06$ & $4.84680 \mathrm{E}-06$ \\
\hline Br-91 & $0.00000 \mathrm{E}+00$ & $0.00000 \mathrm{E}+00$ & $0.00000 \mathrm{E}+00$ & $4.55600 \mathrm{E}-07$ & $4.58531 \mathrm{E}-07$ & 4.62074E-07 \\
\hline Br-92 & $0.00000 \mathrm{E}+00$ & $0.00000 \mathrm{E}+00$ & $0.00000 \mathrm{E}+00$ & $3.69616 \mathrm{E}-08$ & $3.73218 \mathrm{E}-08$ & $3.75784 \mathrm{E}-08$ \\
\hline$B r-93$ & $0.00000 \mathrm{E}+00$ & $0.00000 \mathrm{E}+00$ & $0.00000 \mathrm{E}+00$ & $3.44941 \mathrm{E}-09$ & $3.48145 \mathrm{E}-09$ & $3.45781 \mathrm{E}-09$ \\
\hline$B r-94$ & $0.00000 \mathrm{E}+00$ & $0.00000 \mathrm{E}+00$ & $0.00000 \mathrm{E}+00$ & $3.41909 \mathrm{E}-10$ & $3.37420 \mathrm{E}-10$ & $3.30752 \mathrm{E}-10$ \\
\hline$B r-95$ & $0.00000 \mathrm{E}+00$ & $0.00000 \mathrm{E}+00$ & $0.00000 \mathrm{E}+00$ & $1.04720 \mathrm{E}-12$ & $1.06752 \mathrm{E}-12$ & $1.06941 \mathrm{E}-12$ \\
\hline$B r-96$ & $0.00000 \mathrm{E}+00$ & $0.00000 \mathrm{E}+00$ & $0.00000 \mathrm{E}+00$ & $3.34929 \mathrm{E}-13$ & 3.39792E-13 & $3.41120 \mathrm{E}-13$ \\
\hline Br-97 & $0.00000 \mathrm{E}+00$ & $0.00000 \mathrm{E}+00$ & $0.00000 \mathrm{E}+00$ & $5.39305 \mathrm{E}-15$ & $5.38812 \mathrm{E}-15$ & $5.25812 \mathrm{E}-15$ \\
\hline$K r-77$ & $0.00000 \mathrm{E}+00$ & $0.00000 \mathrm{E}+00$ & $0.00000 \mathrm{E}+00$ & $3.41603 \mathrm{E}-23$ & $3.90281 \mathrm{E}-23$ & $4.09551 \mathrm{E}-23$ \\
\hline$K r-79$ & $0.00000 \mathrm{E}+00$ & $0.00000 \mathrm{E}+00$ & $0.00000 \mathrm{E}+00$ & $4.08348 \mathrm{E}-12$ & 4.08923E-12 & $4.01025 \mathrm{E}-12$ \\
\hline
\end{tabular}




\section{Depletion Analysis of Modular High Temperature Gas-cooled Reactor Loaded with LEU/Thorium Fuel}

February 2012

\begin{tabular}{|c|c|c|c|c|c|c|}
\hline \multirow[b]{2}{*}{ Nuclide } & \multicolumn{3}{|c|}{ Fresh Fuel } & \multicolumn{3}{|c|}{ Discharge } \\
\hline & Unit Cell & $\begin{array}{l}\text { Full Core } \\
(N O L B P)\end{array}$ & Full Core (3) & Unit Cell & $\begin{array}{l}\text { Full Core } \\
\text { (NO LBP) }\end{array}$ & Full Core (3) \\
\hline$r-79 m$ & $0.00000 \mathrm{E}+00$ & $0.00000 \mathrm{E}+00$ & $0.00000 \mathrm{E}+00$ & $7.76604 \mathrm{E}-16$ & $7.71528 \mathrm{E}-16$ & 7.56137E-16 \\
\hline$K r-81$ & $0.00000 \mathrm{E}+00$ & $0.00000 \mathrm{E}+00$ & $0.00000 \mathrm{E}+00$ & $2.94227 \mathrm{E}-05$ & $2.97399 \mathrm{E}-05$ & $3.07574 \mathrm{E}-05$ \\
\hline$K r-81 m$ & $0.00000 \mathrm{E}+00$ & $0.00000 \mathrm{E}+00$ & $0.00000 \mathrm{E}+00$ & $6.70264 \mathrm{E}-13$ & $6.62819 \mathrm{E}-13$ & $6.48866 \mathrm{E}-13$ \\
\hline$K r-83 m$ & $0.00000 \mathrm{E}+00$ & $0.00000 \mathrm{E}+00$ & $0.00000 \mathrm{E}+00$ & $1.98076 \mathrm{E}-02$ & $1.99606 \mathrm{E}-02$ & $1.99377 \mathrm{E}-02$ \\
\hline$K r-85 m$ & $0.00000 \mathrm{E}+00$ & $0.00000 \mathrm{E}+00$ & $0.00000 \mathrm{E}+00$ & 7.24615E-02 & 7.31057E-02 & $7.30458 \mathrm{E}-02$ \\
\hline$K r-87$ & $0.00000 \mathrm{E}+00$ & $0.00000 \mathrm{E}+00$ & $0.00000 \mathrm{E}+00$ & $6.15845 \mathrm{E}-02$ & $6.20680 \mathrm{E}-02$ & $6.20983 \mathrm{E}-02$ \\
\hline$K r-88$ & $0.00000 \mathrm{E}+00$ & $0.00000 \mathrm{E}+00$ & $0.00000 \mathrm{E}+00$ & $1.88313 \mathrm{E}-01$ & $1.89751 \mathrm{E}-01$ & $1.89929 \mathrm{E}-01$ \\
\hline$K r-89$ & $0.00000 \mathrm{E}+00$ & $0.00000 \mathrm{E}+00$ & $0.00000 \mathrm{E}+00$ & $4.22331 \mathrm{E}-03$ & 4.25191E-03 & 4.26042E-03 \\
\hline$K r-90$ & $0.00000 \mathrm{E}+00$ & $0.00000 \mathrm{E}+00$ & $0.00000 \mathrm{E}+00$ & $7.34861 \mathrm{E}-04$ & 7.39181E-04 & 7.41769E-04 \\
\hline$K r-91$ & $0.00000 \mathrm{E}+00$ & $0.00000 \mathrm{E}+00$ & $0.00000 \mathrm{E}+00$ & $1.24835 \mathrm{E}-04$ & $1.25464 \mathrm{E}-04$ & $1.26090 \mathrm{E}-04$ \\
\hline$K r-92$ & $0.00000 \mathrm{E}+00$ & $0.00000 \mathrm{E}+00$ & $0.00000 \mathrm{E}+00$ & $1.31371 \mathrm{E}-05$ & $1.31973 \mathrm{E}-05$ & $1.32740 \mathrm{E}-05$ \\
\hline$K r-93$ & $0.00000 \mathrm{E}+00$ & $0.00000 \mathrm{E}+00$ & $0.00000 \mathrm{E}+00$ & $2.60324 \mathrm{E}-06$ & $2.61675 \mathrm{E}-06$ & $2.63420 \mathrm{E}-06$ \\
\hline$K r-94$ & $0.00000 \mathrm{E}+00$ & $0.00000 \mathrm{E}+00$ & $0.00000 \mathrm{E}+00$ & $8.52617 \mathrm{E}-08$ & $8.58192 \mathrm{E}-08$ & $.62315 \mathrm{E}-08$ \\
\hline$K r-95$ & $0.00000 \mathrm{E}+00$ & $0.00000 \mathrm{E}+00$ & $0.00000 \mathrm{E}+00$ & $3.92662 \mathrm{E}-09$ & 3.97287E-09 & $3.99690 \mathrm{E}-09$ \\
\hline$K r-96$ & $0.00000 \mathrm{E}+00$ & $0.00000 \mathrm{E}+00$ & $0.00000 \mathrm{E}+00$ & $1.12627 \mathrm{E}-08$ & $1.12818 \mathrm{E}-08$ & $1.13703 \mathrm{E}-08$ \\
\hline$K r-97$ & $0.00000 \mathrm{E}+00$ & $0.00000 \mathrm{E}+00$ & $0.00000 \mathrm{E}+00$ & $1.69680 \mathrm{E}-10$ & $1.66333 \mathrm{E}-10$ & $1.62626 \mathrm{E}-10$ \\
\hline$K r-98$ & $0.00000 \mathrm{E}+00$ & $0.00000 \mathrm{E}+00$ & $0.00000 \mathrm{E}+00$ & $2.57702 \mathrm{E}-10$ & $2.58580 \mathrm{E}-10$ & $2.61452 \mathrm{E}-10$ \\
\hline$K r-99$ & $0.00000 \mathrm{E}+00$ & $0.00000 \mathrm{E}+00$ & $0.00000 \mathrm{E}+00$ & $1.23202 \mathrm{E}-14$ & $1.50515 \mathrm{E}-14$ & $1.46205 \mathrm{E}-14$ \\
\hline$K r-100$ & $0.00000 \mathrm{E}+00$ & $0.00000 \mathrm{E}+00$ & $0.00000 \mathrm{E}+00$ & $2.09098 \mathrm{E}-13$ & $2.09834 \mathrm{E}-13$ & $2.12167 \mathrm{E}-13$ \\
\hline$R b-79$ & $0.00000 \mathrm{E}+00$ & $0.00000 \mathrm{E}+00$ & $0.00000 \mathrm{E}+00$ & $0.00000 \mathrm{E}+00$ & $0.00000 \mathrm{E}+00$ & $0.00000 \mathrm{E}+00$ \\
\hline$R b-81$ & $0.00000 \mathrm{E}+00$ & $0.00000 \mathrm{E}+00$ & $0.00000 \mathrm{E}+00$ & $1.50881 \mathrm{E}-13$ & $1.48306 \mathrm{E}-13$ & $1.44962 \mathrm{E}-13$ \\
\hline$R b-83$ & $0.00000 \mathrm{E}+00$ & $0.00000 \mathrm{E}+00$ & $0.00000 \mathrm{E}+00$ & $6.26675 \mathrm{E}-07$ & $6.17666 \mathrm{E}-07$ & $6.13784 \mathrm{E}-07$ \\
\hline$R b-84$ & $0.00000 \mathrm{E}+00$ & $0.00000 \mathrm{E}+00$ & $0.00000 \mathrm{E}+00$ & $3.30688 \mathrm{E}-06$ & 3.79153E-06 & $3.83358 \mathrm{E}-06$ \\
\hline$R b-86 m$ & $0.00000 \mathrm{E}+00$ & $0.00000 \mathrm{E}+00$ & $0.00000 \mathrm{E}+00$ & $3.73982 \mathrm{E}-08$ & $3.80278 \mathrm{E}-08$ & $3.75168 \mathrm{E}-08$ \\
\hline$R b-88$ & $0.00000 \mathrm{E}+00$ & $0.00000 \mathrm{E}+00$ & $0.00000 \mathrm{E}+00$ & $2.02113 \mathrm{E}-02$ & $2.03721 \mathrm{E}-02$ & 2.03834E-02 \\
\hline$R b-89$ & $0.00000 \mathrm{E}+00$ & $0.00000 \mathrm{E}+00$ & $0.00000 \mathrm{E}+00$ & $2.22059 \mathrm{E}-02$ & $2.23637 \mathrm{E}-02$ & $2.23918 \mathrm{E}-02$ \\
\hline$R b-90$ & $0.00000 \mathrm{E}+00$ & $0.00000 \mathrm{E}+00$ & $0.00000 \mathrm{E}+00$ & $3.79354 \mathrm{E}-03$ & $3.81620 \mathrm{E}-03$ & 3.82793E-03 \\
\hline$R b-90 m$ & $0.00000 \mathrm{E}+00$ & $0.00000 \mathrm{E}+00$ & $0.00000 \mathrm{E}+00$ & $1.34675 \mathrm{E}-03$ & $1.35688 \mathrm{E}-03$ & $1.35196 \mathrm{E}-03$ \\
\hline$R b-91$ & $0.00000 \mathrm{E}+00$ & $0.00000 \mathrm{E}+00$ & $0.00000 \mathrm{E}+00$ & $1.62380 \mathrm{E}-03$ & $1.63219 \mathrm{E}-03$ & $1.63590 \mathrm{E}-03$ \\
\hline$R b-92$ & $0.00000 \mathrm{E}+00$ & $0.00000 \mathrm{E}+00$ & $0.00000 \mathrm{E}+00$ & $1.05994 \mathrm{E}-04$ & $1.06381 \mathrm{E}-04$ & $1.06679 \mathrm{E}-04$ \\
\hline$R b-93$ & $0.00000 \mathrm{E}+00$ & $0.00000 \mathrm{E}+00$ & $0.00000 \mathrm{E}+00$ & $9.95749 \mathrm{E}-05$ & $9.98291 \mathrm{E}-05$ & $1.00179 \mathrm{E}-04$ \\
\hline$R b-94$ & $0.00000 \mathrm{E}+00$ & $0.00000 \mathrm{E}+00$ & $0.00000 \mathrm{E}+00$ & $2.17385 \mathrm{E}-05$ & $2.17543 \mathrm{E}-05$ & $2.18410 \mathrm{E}-05$ \\
\hline$R b-95$ & $0.00000 \mathrm{E}+00$ & $0.00000 \mathrm{E}+00$ & $0.00000 \mathrm{E}+00$ & $1.45664 \mathrm{E}-06$ & $1.45601 \mathrm{E}-06$ & $1.46075 \mathrm{E}-06$ \\
\hline$R b-96$ & $0.00000 \mathrm{E}+00$ & $0.00000 \mathrm{E}+00$ & $0.00000 \mathrm{E}+00$ & $1.89642 \mathrm{E}-07$ & $1.90239 \mathrm{E}-07$ & 1.91477E-07 \\
\hline$R b-97$ & $0.00000 \mathrm{E}+00$ & $0.00000 \mathrm{E}+00$ & $0.00000 \mathrm{E}+00$ & $2.95369 \mathrm{E}-08$ & $2.95825 \mathrm{E}-08$ & $2.97937 \mathrm{E}-08$ \\
\hline$R b-98$ & $0.00000 \mathrm{E}+00$ & $0.00000 \mathrm{E}+00$ & $0.00000 \mathrm{E}+00$ & 4.63141E-09 & $4.72602 \mathrm{E}-09$ & 4.70742E-09 \\
\hline
\end{tabular}




\begin{tabular}{|c|c|c|c|c|c|c|}
\hline \multirow[b]{2}{*}{ Nuclide } & \multicolumn{3}{|c|}{ Fresh Fuel } & \multicolumn{3}{|c|}{ Discharge } \\
\hline & Unit Cell & $\begin{array}{l}\text { Full Core } \\
\text { (NO LBP) }\end{array}$ & Full Core (3) & Unit Cell & $\begin{array}{l}\text { Full Core } \\
(N O L B P)\end{array}$ & Full Core (3) \\
\hline$R b-99$ & $0.00000 \mathrm{E}+00$ & $0.00000 \mathrm{E}+00$ & $0.00000 \mathrm{E}+00$ & $2.88647 \mathrm{E}-11$ & $3.18534 \mathrm{E}-11$ & $3.14074 \mathrm{E}-11$ \\
\hline$R b-100$ & $0.00000 \mathrm{E}+00$ & $0.00000 \mathrm{E}+00$ & $0.00000 \mathrm{E}+00$ & $6.19148 \mathrm{E}-09$ & $6.21157 \mathrm{E}-09$ & $6.28100 \mathrm{E}-09$ \\
\hline$R b-101$ & $0.00000 \mathrm{E}+00$ & $0.00000 \mathrm{E}+00$ & $0.00000 \mathrm{E}+00$ & $2.32329 \mathrm{E}-13$ & $2.36743 \mathrm{E}-13$ & $2.38324 \mathrm{E}-13$ \\
\hline$R b-102$ & $0.00000 \mathrm{E}+00$ & $0.00000 \mathrm{E}+00$ & $0.00000 \mathrm{E}+00$ & $3.19975 \mathrm{E}-15$ & $3.87984 \mathrm{E}-15$ & $3.77720 \mathrm{E}-15$ \\
\hline$S r-83$ & $0.00000 \mathrm{E}+00$ & $0.00000 \mathrm{E}+00$ & $0.00000 \mathrm{E}+00$ & $1.89491 \mathrm{E}-13$ & $1.87171 \mathrm{E}-13$ & $1.83230 \mathrm{E}-13$ \\
\hline$S r-85$ & $0.00000 \mathrm{E}+00$ & $0.00000 \mathrm{E}+00$ & $0.00000 \mathrm{E}+00$ & $1.36267 \mathrm{E}-07$ & $1.37371 \mathrm{E}-07$ & $1.36316 \mathrm{E}-07$ \\
\hline$S r-85 m$ & $0.00000 \mathrm{E}+00$ & $0.00000 \mathrm{E}+00$ & $0.00000 \mathrm{E}+00$ & $5.22780 \mathrm{E}-11$ & $5.22766 \mathrm{E}-11$ & $5.13084 \mathrm{E}-11$ \\
\hline$S r-87 m$ & $0.00000 \mathrm{E}+00$ & $0.00000 \mathrm{E}+00$ & $0.00000 \mathrm{E}+00$ & $1.61051 \mathrm{E}-07$ & $1.61319 \mathrm{E}-07$ & $1.58600 \mathrm{E}-07$ \\
\hline Sr-91 & $0.00000 \mathrm{E}+00$ & $0.00000 \mathrm{E}+00$ & $0.00000 \mathrm{E}+00$ & $1.05416 \mathrm{E}+00$ & $1.05997 \mathrm{E}+00$ & $1.06151 \mathrm{E}+00$ \\
\hline Sr-92 & $0.00000 \mathrm{E}+00$ & $0.00000 \mathrm{E}+00$ & $0.00000 \mathrm{E}+00$ & $3.03085 \mathrm{E}-01$ & $3.04449 \mathrm{E}-01$ & $3.04782 \mathrm{E}-01$ \\
\hline$S r-93$ & $0.00000 \mathrm{E}+00$ & $0.00000 \mathrm{E}+00$ & $0.00000 \mathrm{E}+00$ & 2101E-02 & $1.52624 \mathrm{E}-02$ & $1 \mathrm{E}-02$ \\
\hline Sr-94 & $0.00000 \mathrm{E}+00$ & $0.00000 \mathrm{E}+00$ & $0.00000 \mathrm{E}+00$ & $2.55785 \mathrm{E}-03$ & $2.56366 \mathrm{E}-03$ & $2.56661 \mathrm{E}-03$ \\
\hline Sr-95 & $0.00000 \mathrm{E}+00$ & $0.00000 \mathrm{E}+00$ & $0.00000 \mathrm{E}+00$ & $6.78384 \mathrm{E}-04$ & $6.79056 \mathrm{E}-04$ & $6.80420 \mathrm{E}-04$ \\
\hline Sr-96 & $0.00000 \mathrm{E}+00$ & $0.00000 \mathrm{E}+00$ & $0.00000 \mathrm{E}+00$ & $2.08145 \mathrm{E}-05$ & $2.08284 \mathrm{E}-05$ & $2.08947 \mathrm{E}-05$ \\
\hline Sr-97 & $0.00000 \mathrm{E}+00$ & $0.00000 \mathrm{E}+00$ & $0.00000 \mathrm{E}+00$ & $3.86633 \mathrm{E}-06$ & $3.86576 \mathrm{E}-06$ & $3.88151 \mathrm{E}-06$ \\
\hline Sr-98 & $0.00000 \mathrm{E}+00$ & $0.00000 \mathrm{E}+00$ & $0.00000 \mathrm{E}+00$ & $2.61111 \mathrm{E}-06$ & $2.61429 \mathrm{E}-06$ & 2.62603E-06 \\
\hline Sr-99 & $0.00000 \mathrm{E}+00$ & $0.00000 \mathrm{E}+00$ & $0.00000 \mathrm{E}+00$ & $1.80982 \mathrm{E}-07$ & $1.81477 \mathrm{E}-07$ & $1.82532 \mathrm{E}-07$ \\
\hline$S r-100$ & $0.00000 \mathrm{E}+00$ & $0.00000 \mathrm{E}+00$ & $0.00000 \mathrm{E}+00$ & $3.76657 \mathrm{E}-08$ & $3.78613 \mathrm{E}-08$ & $3.81414 \mathrm{E}-08$ \\
\hline Sr-101 & $0.00000 \mathrm{E}+00$ & $0.00000 \mathrm{E}+00$ & $0.00000 \mathrm{E}+00$ & $2.24139 \mathrm{E}-09$ & $2.26119 \mathrm{E}-09$ & $2.27951 \mathrm{E}-09$ \\
\hline$S r-102$ & $0.00000 \mathrm{E}+00$ & $0.00000 \mathrm{E}+00$ & $0.00000 \mathrm{E}+00$ & $5.95982 \mathrm{E}-11$ & $6.10080 \mathrm{E}-11$ & $6.13127 \mathrm{E}-11$ \\
\hline$S r-103$ & $0.00000 \mathrm{E}+00$ & $0.00000 \mathrm{E}+00$ & $0.00000 \mathrm{E}+00$ & $1.55033 \mathrm{E}-12$ & $1.57638 \mathrm{E}-12$ & $1.57665 \mathrm{E}-12$ \\
\hline$S r-104$ & $0.00000 \mathrm{E}+00$ & $0.00000 \mathrm{E}+00$ & $0.00000 \mathrm{E}+00$ & $6.16625 \mathrm{E}-14$ & $6.64349 \mathrm{E}-14$ & $6.60937 \mathrm{E}-14$ \\
\hline$S r-105$ & $0.00000 \mathrm{E}+00$ & $0.00000 \mathrm{E}+00$ & $0.00000 \mathrm{E}+00$ & $3.91963 \mathrm{E}-15$ & 4.83364E-15 & 4.69149E-15 \\
\hline$Y-85$ & $0.00000 \mathrm{E}+00$ & $0.00000 \mathrm{E}+00$ & $0.00000 \mathrm{E}+00$ & $7.84931 \mathrm{E}-17$ & $9.63849 \mathrm{E}-17$ & $1.00746 \mathrm{E}-16$ \\
\hline$Y-87$ & $0.00000 \mathrm{E}+00$ & $0.00000 \mathrm{E}+00$ & $0.00000 \mathrm{E}+00$ & $1.58763 \mathrm{E}-09$ & $1.57672 \mathrm{E}-09$ & $1.54549 \mathrm{E}-09$ \\
\hline$Y-87 m$ & $0.00000 \mathrm{E}+00$ & $0.00000 \mathrm{E}+00$ & $0.00000 \mathrm{E}+00$ & $7.73329 \mathrm{E}-17$ & $9.29545 \mathrm{E}-17$ & $9.73842 \mathrm{E}-17$ \\
\hline$Y-88$ & $0.00000 \mathrm{E}+00$ & $0.00000 \mathrm{E}+00$ & $0.00000 \mathrm{E}+00$ & $2.75642 \mathrm{E}-05$ & $3.11969 \mathrm{E}-05$ & $3.12627 \mathrm{E}-05$ \\
\hline$Y-89 m$ & $0.00000 \mathrm{E}+00$ & $0.00000 \mathrm{E}+00$ & $0.00000 \mathrm{E}+00$ & $3.93337 \mathrm{E}-08$ & $3.96464 \mathrm{E}-08$ & $3.96478 \mathrm{E}-08$ \\
\hline$Y-90 m$ & $0.00000 \mathrm{E}+00$ & $0.00000 \mathrm{E}+00$ & $0.00000 \mathrm{E}+00$ & $2.65722 \mathrm{E}-06$ & $2.64739 \mathrm{E}-06$ & 2.60053E-06 \\
\hline$Y-91 m$ & $0.00000 \mathrm{E}+00$ & $0.00000 \mathrm{E}+00$ & $0.00000 \mathrm{E}+00$ & $5.19202 \mathrm{E}-02$ & $5.22062 \mathrm{E}-02$ & $5.22820 \mathrm{E}-02$ \\
\hline$Y-92$ & $0.00000 \mathrm{E}+00$ & $0.00000 \mathrm{E}+00$ & $0.00000 \mathrm{E}+00$ & 4.09617E-01 & 4.11494E-01 & 4.11915E-01 \\
\hline$Y-93$ & $0.00000 \mathrm{E}+00$ & $0.00000 \mathrm{E}+00$ & $0.00000 \mathrm{E}+00$ & $1.29535 \mathrm{E}+00$ & $1.30004 \mathrm{E}+00$ & $1.30060 \mathrm{E}+00$ \\
\hline$Y-93 m$ & $0.00000 \mathrm{E}+00$ & $0.00000 \mathrm{E}+00$ & $0.00000 \mathrm{E}+00$ & $1.96447 \mathrm{E}-06$ & $1.97374 \mathrm{E}-06$ & $1.97116 \mathrm{E}-06$ \\
\hline$Y-94$ & $0.00000 \mathrm{E}+00$ & $0.00000 \mathrm{E}+00$ & $0.00000 \mathrm{E}+00$ & $4.24419 \mathrm{E}-02$ & $4.25499 \mathrm{E}-02$ & $4.25638 \mathrm{E}-02$ \\
\hline$Y-95$ & $0.00000 \mathrm{E}+00$ & $0.00000 \mathrm{E}+00$ & $0.00000 \mathrm{E}+00$ & $2.33984 \mathrm{E}-02$ & $2.34299 \mathrm{E}-02$ & $2.34338 \mathrm{E}-02$ \\
\hline$Y-96$ & $0.00000 \mathrm{E}+00$ & $0.00000 \mathrm{E}+00$ & $0.00000 \mathrm{E}+00$ & $1.12181 \mathrm{E}-04$ & $1.12248 \mathrm{E}-04$ & $1.12560 \mathrm{E}-04$ \\
\hline
\end{tabular}




\section{Depletion Analysis of Modular High Temperature Gas-cooled Reactor Loaded with LEU/Thorium Fuel}

February 2012

\begin{tabular}{|c|c|c|c|c|c|c|}
\hline \multirow[b]{2}{*}{ Nuclide } & \multicolumn{3}{|c|}{ Fresh Fuel } & \multicolumn{3}{|c|}{ Discharge } \\
\hline & Unit Cell & $\begin{array}{l}\text { Full Core } \\
(N O L B P)\end{array}$ & Full Core (3) & Unit Cell & $\begin{array}{l}\text { Full Core } \\
(N O L B P)\end{array}$ & Full Core (3) \\
\hline$Y-96 m$ & $0.00000 \mathrm{E}+00$ & $0.00000 \mathrm{E}+00$ & $0.00000 \mathrm{E}+00$ & $1.34589 \mathrm{E}-04$ & $1.34563 \mathrm{E}-04$ & $1.34261 \mathrm{E}-04$ \\
\hline$Y-97$ & $0.00000 \mathrm{E}+00$ & $0.00000 \mathrm{E}+00$ & $0.00000 \mathrm{E}+00$ & $7.16296 \mathrm{E}-05$ & $7.15726 \mathrm{E}-05$ & 7.16929E-05 \\
\hline$Y-97 m$ & $0.00000 \mathrm{E}+00$ & $0.00000 \mathrm{E}+00$ & $0.00000 \mathrm{E}+00$ & $1.17228 \mathrm{E}-05$ & $1.17067 \mathrm{E}-05$ & $1.17012 \mathrm{E}-05$ \\
\hline$Y-98$ & $0.00000 \mathrm{E}+00$ & $0.00000 \mathrm{E}+00$ & $0.00000 \mathrm{E}+00$ & 7.05053E-06 & 7.03367E-06 & $7.04205 \mathrm{E}-06$ \\
\hline$Y-98 m$ & $0.00000 \mathrm{E}+00$ & $0.00000 \mathrm{E}+00$ & $0.00000 \mathrm{E}+00$ & $1.47792 \mathrm{E}-05$ & $1.47197 \mathrm{E}-05$ & $1.47151 \mathrm{E}-05$ \\
\hline$Y-99$ & $0.00000 \mathrm{E}+00$ & $0.00000 \mathrm{E}+00$ & $0.00000 \mathrm{E}+00$ & $1.78538 \mathrm{E}-05$ & $1.78038 \mathrm{E}-05$ & $1.78457 \mathrm{E}-05$ \\
\hline$Y-100$ & $0.00000 \mathrm{E}+00$ & $0.00000 \mathrm{E}+00$ & $0.00000 \mathrm{E}+00$ & $2.69359 \mathrm{E}-06$ & $2.68488 \mathrm{E}-06$ & $2.69571 \mathrm{E}-06$ \\
\hline$Y-101$ & $0.00000 \mathrm{E}+00$ & $0.00000 \mathrm{E}+00$ & $0.00000 \mathrm{E}+00$ & $6.56054 \mathrm{E}-07$ & $6.56217 \mathrm{E}-07$ & $6.60188 \mathrm{E}-07$ \\
\hline$Y-102$ & $0.00000 \mathrm{E}+00$ & $0.00000 \mathrm{E}+00$ & $0.00000 \mathrm{E}+00$ & $3.10618 \mathrm{E}-07$ & $3.11568 \mathrm{E}-07$ & $3.14782 \mathrm{E}-07$ \\
\hline$Y-103$ & $0.00000 \mathrm{E}+00$ & $0.00000 \mathrm{E}+00$ & $0.00000 \mathrm{E}+00$ & 4.80885E-09 & 4.86798E-09 & $.88241 \mathrm{E}-09$ \\
\hline$Y-104$ & $0.00000 \mathrm{E}+00$ & $0.00000 \mathrm{E}+00$ & $0.00000 \mathrm{E}+00$ & $5.44002 \mathrm{E}-10$ & $5.51805 \mathrm{E}-10$ & $54982 \mathrm{E}-10$ \\
\hline$Y-105$ & $0.00000 \mathrm{E}+00$ & $0.00000 \mathrm{E}+00$ & $0.00000 \mathrm{E}+00$ & $7.24012 \mathrm{E}-12$ & $8.32602 \mathrm{E}-12$ & $14185 \mathrm{E}-12$ \\
\hline$Y-106$ & $0.00000 \mathrm{E}+00$ & $0.00000 \mathrm{E}+00$ & $0.00000 \mathrm{E}+00$ & $2980 \mathrm{E}-13$ & $1.32971 \mathrm{E}-13$ & $33670 \mathrm{E}-13$ \\
\hline$Y-107$ & $0.00000 \mathrm{E}+00$ & $0.00000 \mathrm{E}+00$ & $0.00000 \mathrm{E}+00$ & $3825 \mathrm{E}-16$ & $1.07569 \mathrm{E}-16$ & $1.07655 \mathrm{E}-16$ \\
\hline$Y-108$ & $0.00000 \mathrm{E}+00$ & $0.00000 \mathrm{E}+00$ & $0.00000 \mathrm{E}+00$ & $1.43174 \mathrm{E}-17$ & $1.58642 \mathrm{E}-17$ & $1.56311 \mathrm{E}-17$ \\
\hline$Z r-87$ & $0.00000 \mathrm{E}+00$ & $0.00000 \mathrm{E}+00$ & $0.00000 \mathrm{E}+00$ & $1.03175 \mathrm{E}-17$ & $1.24018 \mathrm{E}-17$ & $1.29912 \mathrm{E}-17$ \\
\hline$Z r-88$ & $0.00000 \mathrm{E}+00$ & $0.00000 \mathrm{E}+00$ & $0.00000 \mathrm{E}+00$ & $6.16054 \mathrm{E}-11$ & $6.08645 \mathrm{E}-11$ & $6.04968 \mathrm{E}-11$ \\
\hline$Z r-89$ & $0.00000 \mathrm{E}+00$ & $0.00000 \mathrm{E}+00$ & $0.00000 \mathrm{E}+00$ & $2.26768 \mathrm{E}-08$ & $2.68122 \mathrm{E}-08$ & $2.67290 \mathrm{E}-08$ \\
\hline$Z r-89 m$ & $0.00000 \mathrm{E}+00$ & $0.00000 \mathrm{E}+00$ & $0.00000 \mathrm{E}+00$ & $3.13957 \mathrm{E}-22$ & $3.45232 \mathrm{E}-22$ & $3.65128 \mathrm{E}-22$ \\
\hline$Z r-90 m$ & $0.00000 \mathrm{E}+00$ & $0.00000 \mathrm{E}+00$ & $0.00000 \mathrm{E}+00$ & $4.92436 \mathrm{E}-14$ & $4.89060 \mathrm{E}-14$ & 4.79192E-14 \\
\hline$Z r-97$ & $0.00000 \mathrm{E}+00$ & $0.00000 \mathrm{E}+00$ & $0.00000 \mathrm{E}+00$ & $2.30710 \mathrm{E}+00$ & $2.30704 \mathrm{E}+00$ & $2.30564 \mathrm{E}+00$ \\
\hline$Z r-98$ & $0.00000 \mathrm{E}+00$ & $0.00000 \mathrm{E}+00$ & $0.00000 \mathrm{E}+00$ & $1.14204 \mathrm{E}-03$ & $1.14068 \mathrm{E}-03$ & $1.13957 \mathrm{E}-03$ \\
\hline$Z r-99$ & $0.00000 \mathrm{E}+00$ & $0.00000 \mathrm{E}+00$ & $0.00000 \mathrm{E}+00$ & $7.70164 \mathrm{E}-05$ & 7.68514E-05 & 7.68514E-05 \\
\hline$Z r-100$ & $0.00000 \mathrm{E}+00$ & $0.00000 \mathrm{E}+00$ & $0.00000 \mathrm{E}+00$ & $2.51673 \mathrm{E}-04$ & $2.51011 \mathrm{E}-04$ & $2.51079 \mathrm{E}-04$ \\
\hline$Z r-101$ & $0.00000 \mathrm{E}+00$ & $0.00000 \mathrm{E}+00$ & $0.00000 \mathrm{E}+00$ & $4.44981 \mathrm{E}-05$ & $4.43427 \mathrm{E}-05$ & 4.44222E-05 \\
\hline$Z r-102$ & $0.00000 \mathrm{E}+00$ & $0.00000 \mathrm{E}+00$ & $0.00000 \mathrm{E}+00$ & $3.46615 \mathrm{E}-05$ & 3.45553E-05 & $3.46760 \mathrm{E}-05$ \\
\hline$Z r-103$ & $0.00000 \mathrm{E}+00$ & $0.00000 \mathrm{E}+00$ & $0.00000 \mathrm{E}+00$ & 4.05003E-06 & 4.03651E-06 & 4.05692E-06 \\
\hline$Z r-104$ & $0.00000 \mathrm{E}+00$ & $0.00000 \mathrm{E}+00$ & $0.00000 \mathrm{E}+00$ & 7.64519E-07 & $7.60785 \mathrm{E}-07$ & $7.63463 \mathrm{E}-07$ \\
\hline$Z r-105$ & $0.00000 \mathrm{E}+00$ & $0.00000 \mathrm{E}+00$ & $0.00000 \mathrm{E}+00$ & 2.80972E-07 & $2.81591 \mathrm{E}-07$ & $2.84390 \mathrm{E}-07$ \\
\hline$Z r-106$ & $0.00000 \mathrm{E}+00$ & $0.00000 \mathrm{E}+00$ & $0.00000 \mathrm{E}+00$ & $8.78000 \mathrm{E}-10$ & $8.51701 \mathrm{E}-10$ & $8.58162 \mathrm{E}-10$ \\
\hline$Z r-107$ & $0.00000 \mathrm{E}+00$ & $0.00000 \mathrm{E}+00$ & $0.00000 \mathrm{E}+00$ & $1.20488 \mathrm{E}-11$ & $1.17651 \mathrm{E}-11$ & $1.17674 \mathrm{E}-11$ \\
\hline$Z r-108$ & $0.00000 \mathrm{E}+00$ & $0.00000 \mathrm{E}+00$ & $0.00000 \mathrm{E}+00$ & $7.95522 \mathrm{E}-13$ & $7.88638 \mathrm{E}-13$ & $7.90853 \mathrm{E}-13$ \\
\hline$Z r-109$ & $0.00000 \mathrm{E}+00$ & $0.00000 \mathrm{E}+00$ & $0.00000 \mathrm{E}+00$ & $2.31770 \mathrm{E}-13$ & $2.34915 \mathrm{E}-13$ & $2.36626 \mathrm{E}-13$ \\
\hline$Z r-110$ & $0.00000 \mathrm{E}+00$ & $0.00000 \mathrm{E}+00$ & $0.00000 \mathrm{E}+00$ & $3.73211 \mathrm{E}-15$ & $3.77819 \mathrm{E}-15$ & $3.81206 \mathrm{E}-15$ \\
\hline$N b-89$ & $0.00000 \mathrm{E}+00$ & $0.00000 \mathrm{E}+00$ & $0.00000 \mathrm{E}+00$ & $1.18991 \mathrm{E}-18$ & $1.30845 \mathrm{E}-18$ & $1.38386 \mathrm{E}-18$ \\
\hline$N b-90$ & $0.00000 \mathrm{E}+00$ & $0.00000 \mathrm{E}+00$ & $0.00000 \mathrm{E}+00$ & $1.15692 \mathrm{E}-15$ & $1.29740 \mathrm{E}-15$ & $1.36931 \mathrm{E}-15$ \\
\hline
\end{tabular}




\begin{tabular}{|c|c|c|c|c|c|c|}
\hline \multirow[b]{2}{*}{ Nuclide } & \multicolumn{3}{|c|}{ Fresh Fuel } & \multicolumn{3}{|c|}{ Discharge } \\
\hline & Unit Cell & $\begin{array}{l}\text { Full Core } \\
\text { (NO LBP) }\end{array}$ & Full Core (3) & Unit Cell & $\begin{array}{l}\text { Full Core } \\
(N O L B P)\end{array}$ & Full Core (3) \\
\hline $\mathrm{Nb}-91$ & $0.00000 \mathrm{E}+00$ & $0.00000 \mathrm{E}+00$ & $0.00000 \mathrm{E}+00$ & $5.42034 \mathrm{E}-09$ & $5.26435 \mathrm{E}-09$ & $5.54961 \mathrm{E}-09$ \\
\hline $\mathrm{Nb}-91 \mathrm{~m}$ & $0.00000 \mathrm{E}+00$ & $0.00000 \mathrm{E}+00$ & $0.00000 \mathrm{E}+00$ & $2.14314 \mathrm{E}-20$ & $2.10549 \mathrm{E}-20$ & $2.28103 \mathrm{E}-20$ \\
\hline $\mathrm{Nb}-92$ & $0.00000 \mathrm{E}+00$ & $0.00000 \mathrm{E}+00$ & $0.00000 \mathrm{E}+00$ & $4.98668 \mathrm{E}-07$ & $4.84839 \mathrm{E}-07$ & $5.10346 \mathrm{E}-07$ \\
\hline$N b-93 m$ & $0.00000 \mathrm{E}+00$ & $0.00000 \mathrm{E}+00$ & $0.00000 \mathrm{E}+00$ & $1.31915 \mathrm{E}-03$ & $1.32205 \mathrm{E}-03$ & $1.31356 \mathrm{E}-03$ \\
\hline$N b-94 m$ & $0.00000 \mathrm{E}+00$ & $0.00000 \mathrm{E}+00$ & $0.00000 \mathrm{E}+00$ & 4.85741E-09 & 4.80217E-09 & 4.70277E-09 \\
\hline Nb-95m & $0.00000 \mathrm{E}+00$ & $0.00000 \mathrm{E}+00$ & $0.00000 \mathrm{E}+00$ & $1.38530 \mathrm{E}-01$ & $1.38880 \mathrm{E}-01$ & $1.38814 \mathrm{E}-01$ \\
\hline$N b-96$ & $0.00000 \mathrm{E}+00$ & $0.00000 \mathrm{E}+00$ & $0.00000 \mathrm{E}+00$ & $1.01924 \mathrm{E}-02$ & $1.05385 \mathrm{E}-02$ & $1.02157 \mathrm{E}-02$ \\
\hline Nb-97 & $0.00000 \mathrm{E}+00$ & $0.00000 \mathrm{E}+00$ & $0.00000 \mathrm{E}+00$ & $1.66793 \mathrm{E}-01$ & $1.66789 \mathrm{E}-01$ & $1.66673 \mathrm{E}-01$ \\
\hline$N b-97 m$ & $0.00000 \mathrm{E}+00$ & $0.00000 \mathrm{E}+00$ & $0.00000 \mathrm{E}+00$ & $32184 \mathrm{E}-06$ & $2.82433 \mathrm{E}-06$ & $8589 \mathrm{E}-06$ \\
\hline$N b-98$ & $0.00000 \mathrm{E}+00$ & $0.00000 \mathrm{E}+00$ & $0.00000 \mathrm{E}+00$ & $8674 \mathrm{E}-04$ & $1.08551 \mathrm{E}-04$ & $0 \mathrm{E}-04$ \\
\hline $\mathrm{Nb}-98 \mathrm{~m}$ & $0.00000 \mathrm{E}+00$ & $0.00000 \mathrm{E}+00$ & $0000 \mathrm{E}+00$ & 9599E-04 & $8.20481 \mathrm{E}-04$ & $6 \mathrm{E}-04$ \\
\hline$N b-99$ & $0.00000 \mathrm{E}+00$ & $0.00000 \mathrm{E}+00$ & $0.00000 \mathrm{E}+00$ & $6893 \mathrm{E}-04$ & $5.55701 \mathrm{E}-04$ & 18E-04 \\
\hline$N b-99 m$ & $0.00000 \mathrm{E}+00$ & $0.00000 \mathrm{E}+00$ & $0.00000 \mathrm{E}+00$ & $.7 \mathrm{E}-04$ & $5.91544 \mathrm{E}-04$ & $9 \mathrm{E}-04$ \\
\hline$N b-100$ & $0.00000 \mathrm{E}+00$ & $0.00000 \mathrm{E}+00$ & $0.00000 \mathrm{E}+00$ & $9123 \mathrm{E}-05$ & $5.77482 \mathrm{E}-05$ & $5.77326 \mathrm{E}-05$ \\
\hline $\mathrm{Nb}-100 \mathrm{~m}$ & $0.00000 \mathrm{E}+00$ & $0.00000 \mathrm{E}+00$ & $0.00000 \mathrm{E}+00$ & $9.45618 \mathrm{E}-06$ & $9.40771 \mathrm{E}-06$ & $9.34803 \mathrm{E}-06$ \\
\hline $\mathrm{Nb}-101$ & $0.00000 \mathrm{E}+00$ & $0.00000 \mathrm{E}+00$ & $0.00000 \mathrm{E}+00$ & $2.48291 \mathrm{E}-04$ & $2.47171 \mathrm{E}-04$ & $2.46947 \mathrm{E}-04$ \\
\hline$N b-102$ & $0.00000 \mathrm{E}+00$ & $0.00000 \mathrm{E}+00$ & $0.00000 \mathrm{E}+00$ & $2.48800 \mathrm{E}-05$ & $2.47487 \mathrm{E}-05$ & $2.47725 \mathrm{E}-05$ \\
\hline$N b-102 m$ & $0.00000 \mathrm{E}+00$ & $0.00000 \mathrm{E}+00$ & $0.00000 \mathrm{E}+00$ & $3.09033 \mathrm{E}-05$ & $3.06264 \mathrm{E}-05$ & $3.05260 \mathrm{E}-05$ \\
\hline$N b-103$ & $0.00000 \mathrm{E}+00$ & $0.00000 \mathrm{E}+00$ & $0.00000 \mathrm{E}+00$ & $2.44794 \mathrm{E}-05$ & $2.42584 \mathrm{E}-05$ & $.42356 \mathrm{E}-05$ \\
\hline Nb-104 & $0.00000 \mathrm{E}+00$ & $0.00000 \mathrm{E}+00$ & $0.00000 \mathrm{E}+00$ & $1.88817 \mathrm{E}-05$ & $1.86422 \mathrm{E}-05$ & $1.86449 \mathrm{E}-05$ \\
\hline $\mathrm{Nb}-104 \mathrm{~m}$ & $0.00000 \mathrm{E}+00$ & $0.00000 \mathrm{E}+00$ & $0.00000 \mathrm{E}+00$ & $3.02335 \mathrm{E}-06$ & $2.98033 \mathrm{E}-06$ & $2.97877 \mathrm{E}-06$ \\
\hline$N b-105$ & $0.00000 \mathrm{E}+00$ & $0.00000 \mathrm{E}+00$ & $0.00000 \mathrm{E}+00$ & $8.38952 \mathrm{E}-06$ & $8.27924 \mathrm{E}-06$ & $.27521 \mathrm{E}-06$ \\
\hline$N b-106$ & $0.00000 \mathrm{E}+00$ & $0.00000 \mathrm{E}+00$ & $0.00000 \mathrm{E}+00$ & $4.56938 \mathrm{E}-07$ & 4.47183E-07 & $01 \mathrm{E}-07$ \\
\hline$N b-107$ & $0.00000 \mathrm{E}+00$ & $0.00000 \mathrm{E}+00$ & $0.00000 \mathrm{E}+00$ & $2.87736 \mathrm{E}-08$ & $2.81946 \mathrm{E}-08$ & 2793E-08 \\
\hline$N b-108$ & $0.00000 \mathrm{E}+00$ & $0.00000 \mathrm{E}+00$ & $0.00000 \mathrm{E}+00$ & $1.60994 \mathrm{E}-09$ & $1.57789 \mathrm{E}-09$ & 91E-09 \\
\hline Nb-109 & $0.00000 \mathrm{E}+00$ & $0.00000 \mathrm{E}+00$ & $0.00000 \mathrm{E}+00$ & $4.34441 \mathrm{E}-10$ & $4.36323 \mathrm{E}-10$ & 4.40504E-10 \\
\hline$N b-110$ & $0.00000 \mathrm{E}+00$ & $0.00000 \mathrm{E}+00$ & $0.00000 \mathrm{E}+00$ & $1.09461 \mathrm{E}-11$ & $1.11271 \mathrm{E}-11$ & $1.12159 \mathrm{E}-11$ \\
\hline$N b-111$ & $0.00000 \mathrm{E}+00$ & $0.00000 \mathrm{E}+00$ & $0.00000 \mathrm{E}+00$ & $5.91175 \mathrm{E}-13$ & $7.23299 \mathrm{E}-13$ & 7.03307E-13 \\
\hline $\mathrm{Nb}-112$ & $0.00000 \mathrm{E}+00$ & $0.00000 \mathrm{E}+00$ & $0.00000 \mathrm{E}+00$ & $2.84838 \mathrm{E}-15$ & $3.35951 \mathrm{E}-15$ & $3.28746 \mathrm{E}-15$ \\
\hline$N b-113$ & $0.00000 \mathrm{E}+00$ & $0.00000 \mathrm{E}+00$ & $0.00000 \mathrm{E}+00$ & $1.67851 \mathrm{E}-16$ & $2.07424 \mathrm{E}-16$ & $2.01236 \mathrm{E}-16$ \\
\hline Mo-90 & $0.00000 \mathrm{E}+00$ & $0.00000 \mathrm{E}+00$ & $0.00000 \mathrm{E}+00$ & $0.00000 \mathrm{E}+00$ & $0.00000 \mathrm{E}+00$ & $0.00000 \mathrm{E}+00$ \\
\hline Mo-91 & $0.00000 \mathrm{E}+00$ & $0.00000 \mathrm{E}+00$ & $0.00000 \mathrm{E}+00$ & $2.29225 \mathrm{E}-20$ & $2.25930 \mathrm{E}-20$ & $2.42043 \mathrm{E}-20$ \\
\hline Mo-93 & $0.00000 \mathrm{E}+00$ & $0.00000 \mathrm{E}+00$ & $0.00000 \mathrm{E}+00$ & $1.04321 \mathrm{E}-09$ & $1.14058 \mathrm{E}-09$ & $1.21439 \mathrm{E}-09$ \\
\hline Mo- $-93 \mathrm{~m}$ & $0.00000 \mathrm{E}+00$ & $0.00000 \mathrm{E}+00$ & $0.00000 \mathrm{E}+00$ & $2.44769 \mathrm{E}-13$ & $2.41483 \mathrm{E}-13$ & $2.36426 \mathrm{E}-13$ \\
\hline Mo-101 & $0.00000 \mathrm{E}+00$ & $0.00000 \mathrm{E}+00$ & $0.00000 \mathrm{E}+00$ & $3.18866 \mathrm{E}-02$ & $3.17613 \mathrm{E}-02$ & $3.17279 \mathrm{E}-02$ \\
\hline Mo-102 & $0.00000 \mathrm{E}+00$ & $0.00000 \mathrm{E}+00$ & $0.00000 \mathrm{E}+00$ & $2.22836 \mathrm{E}-02$ & $2.21449 \mathrm{E}-02$ & $2.21071 \mathrm{E}-02$ \\
\hline
\end{tabular}




\section{Depletion Analysis of Modular High Temperature Gas-cooled Reactor Loaded with LEU/Thorium Fuel}

February 2012

53

\begin{tabular}{|c|c|c|c|c|c|c|}
\hline \multirow[b]{2}{*}{ Nuclide } & \multicolumn{3}{|c|}{ Fresh Fuel } & \multicolumn{3}{|c|}{ Discharge } \\
\hline & Unit Cell & $\begin{array}{l}\text { Full Core } \\
(N O L B P)\end{array}$ & Full Core (3) & Unit Cell & $\begin{array}{l}\text { Full Core } \\
\text { (NO LBP) }\end{array}$ & Full Core (3) \\
\hline Mo-103 & $0.00000 \mathrm{E}+00$ & $0.00000 \mathrm{E}+00$ & $0.00000 \mathrm{E}+00$ & $1.92732 \mathrm{E}-03$ & $1.90815 \mathrm{E}-03$ & $1.89988 \mathrm{E}-03$ \\
\hline Mo-104 & $0.00000 \mathrm{E}+00$ & $0.00000 \mathrm{E}+00$ & $0.00000 \mathrm{E}+00$ & $1.31412 \mathrm{E}-03$ & $1.29676 \mathrm{E}-03$ & $1.29011 \mathrm{E}-03$ \\
\hline Mo-105 & $0.00000 \mathrm{E}+00$ & $0.00000 \mathrm{E}+00$ & $0.00000 \mathrm{E}+00$ & $5.23721 \mathrm{E}-04$ & 5.15351E-04 & $5.12156 \mathrm{E}-04$ \\
\hline Mo-106 & $0.00000 \mathrm{E}+00$ & $0.00000 \mathrm{E}+00$ & $0.00000 \mathrm{E}+00$ & $7.20227 \mathrm{E}-05$ & $7.06338 \mathrm{E}-05$ & $7.02635 \mathrm{E}-05$ \\
\hline Mo-107 & $0.00000 \mathrm{E}+00$ & $0.00000 \mathrm{E}+00$ & $0.00000 \mathrm{E}+00$ & $1.11294 \mathrm{E}-05$ & $1.09005 \mathrm{E}-05$ & $1.08866 \mathrm{E}-05$ \\
\hline Mo-108 & $0.00000 \mathrm{E}+00$ & $0.00000 \mathrm{E}+00$ & $0.00000 \mathrm{E}+00$ & $1.15442 \mathrm{E}-06$ & $1.13118 \mathrm{E}-06$ & $1.12786 \mathrm{E}-06$ \\
\hline Mo-109 & $0.00000 \mathrm{E}+00$ & $0.00000 \mathrm{E}+00$ & $0.00000 \mathrm{E}+00$ & $9.12246 \mathrm{E}-08$ & $9.01128 \mathrm{E}-08$ & $9.07654 \mathrm{E}-08$ \\
\hline Mo-110 & $0.00000 \mathrm{E}+00$ & $0.00000 \mathrm{E}+00$ & $0.00000 \mathrm{E}+00$ & 4.77046E-09 & 4.87078E-09 & 4.90123E-09 \\
\hline Mo-111 & $0.00000 \mathrm{E}+00$ & $0.00000 \mathrm{E}+00$ & $0.00000 \mathrm{E}+00$ & $2.94531 \mathrm{E}-10$ & $3.12691 \mathrm{E}-10$ & $12303 \mathrm{E}-10$ \\
\hline Mo-112 & $0.00000 \mathrm{E}+00$ & $0.00000 \mathrm{E}+00$ & $0.00000 \mathrm{E}+00$ & $2.27815 \mathrm{E}-11$ & $2.54750 \mathrm{E}-11$ & $51697 \mathrm{E}-11$ \\
\hline Mo-113 & $0.00000 \mathrm{E}+00$ & $0.00000 \mathrm{E}+00$ & $0.00000 \mathrm{E}+00$ & 4.80727E-13 & $5.75538 \mathrm{E}-13$ & $.61031 \mathrm{E}-13$ \\
\hline Mo-114 & $0.00000 \mathrm{E}+00$ & $0.00000 \mathrm{E}+00$ & $0.00000 \mathrm{E}+00$ & $1.71694 \mathrm{E}-13$ & $1.74008 \mathrm{E}-13$ & 69819E-13 \\
\hline Mo-115 & $0.00000 \mathrm{E}+00$ & $0.00000 \mathrm{E}+00$ & $0.00000 \mathrm{E}+00$ & $8.24940 \mathrm{E}-15$ & $8.06133 \mathrm{E}-15$ & $8.13747 \mathrm{E}-15$ \\
\hline$T c-93$ & $0.00000 \mathrm{E}+00$ & $0.00000 \mathrm{E}+00$ & $0.00000 \mathrm{E}+00$ & $0.00000 \mathrm{E}+00$ & $0.00000 \mathrm{E}+00$ & $0.00000 \mathrm{E}+00$ \\
\hline$T c-94$ & $0.00000 \mathrm{E}+00$ & $0.00000 \mathrm{E}+00$ & $0.00000 \mathrm{E}+00$ & $0.00000 \mathrm{E}+00$ & $0.00000 \mathrm{E}+00$ & $0.00000 \mathrm{E}+00$ \\
\hline$T c-95$ & $0.00000 \mathrm{E}+00$ & $0.00000 \mathrm{E}+00$ & $0.00000 \mathrm{E}+00$ & $4.46724 \mathrm{E}-15$ & 4.55644E-15 & 4.86219E-15 \\
\hline$T c-95 m$ & $0.00000 \mathrm{E}+00$ & $0.00000 \mathrm{E}+00$ & $0.00000 \mathrm{E}+00$ & $5.42160 \mathrm{E}-14$ & $5.54319 \mathrm{E}-14$ & 5.97817E-14 \\
\hline Tc-96 & $0.00000 \mathrm{E}+00$ & $0.00000 \mathrm{E}+00$ & $0.00000 \mathrm{E}+00$ & $7.08001 \mathrm{E}-22$ & $1.06177 \mathrm{E}-21$ & $1.09486 \mathrm{E}-21$ \\
\hline Tc-97 & $0.00000 \mathrm{E}+00$ & $0.00000 \mathrm{E}+00$ & $0.00000 \mathrm{E}+00$ & $5.34019 \mathrm{E}-07$ & $5.18168 \mathrm{E}-07$ & $5.49088 \mathrm{E}-07$ \\
\hline$T c-97 m$ & $0.00000 \mathrm{E}+00$ & $0.00000 \mathrm{E}+00$ & $0.00000 \mathrm{E}+00$ & $5.68038 \mathrm{E}-08$ & $5.57350 \mathrm{E}-08$ & $5.54160 \mathrm{E}-08$ \\
\hline Tc-98 & $0.00000 \mathrm{E}+00$ & $0.00000 \mathrm{E}+00$ & $0.00000 \mathrm{E}+00$ & $1.38996 \mathrm{E}-03$ & $1.60693 \mathrm{E}-03$ & $1.66629 \mathrm{E}-03$ \\
\hline$T c-99 m$ & $0.00000 \mathrm{E}+00$ & $0.00000 \mathrm{E}+00$ & $0.00000 \mathrm{E}+00$ & $7.89559 \mathrm{E}-01$ & $7.88216 \mathrm{E}-01$ & 7.87572E-01 \\
\hline$T c-100$ & $0.00000 \mathrm{E}+00$ & $0.00000 \mathrm{E}+00$ & $0.00000 \mathrm{E}+00$ & $1.56885 \mathrm{E}-04$ & $1.56247 \mathrm{E}-04$ & $1.51019 \mathrm{E}-04$ \\
\hline$T c-101$ & $0.00000 \mathrm{E}+00$ & $0.00000 \mathrm{E}+00$ & $0.00000 \mathrm{E}+00$ & $3.10539 \mathrm{E}-02$ & 3.09316E-02 & $3.08986 \mathrm{E}-02$ \\
\hline$T c-102$ & $0.00000 \mathrm{E}+00$ & $0.00000 \mathrm{E}+00$ & $0.00000 \mathrm{E}+00$ & $1.74046 \mathrm{E}-04$ & $1.72960 \mathrm{E}-04$ & $1.72660 \mathrm{E}-04$ \\
\hline$T c-102 m$ & $0.00000 \mathrm{E}+00$ & $0.00000 \mathrm{E}+00$ & $0.00000 \mathrm{E}+00$ & $2.47998 \mathrm{E}-05$ & $2.45349 \mathrm{E}-05$ & $.42802 \mathrm{E}-05$ \\
\hline$T c-103$ & $0.00000 \mathrm{E}+00$ & $0.00000 \mathrm{E}+00$ & $0.00000 \mathrm{E}+00$ & $1.59071 \mathrm{E}-03$ & $1.57492 \mathrm{E}-03$ & $1.56788 \mathrm{E}-03$ \\
\hline$T c-104$ & $0.00000 \mathrm{E}+00$ & $0.00000 \mathrm{E}+00$ & $0.00000 \mathrm{E}+00$ & $2.56598 \mathrm{E}-02$ & $2.53189 \mathrm{E}-02$ & $2.51783 \mathrm{E}-02$ \\
\hline$T c-105$ & $0.00000 \mathrm{E}+00$ & $0.00000 \mathrm{E}+00$ & $0.00000 \mathrm{E}+00$ & $8.27058 \mathrm{E}-03$ & $8.13119 \mathrm{E}-03$ & $8.06371 \mathrm{E}-03$ \\
\hline$T c-106$ & $0.00000 \mathrm{E}+00$ & $0.00000 \mathrm{E}+00$ & $0.00000 \mathrm{E}+00$ & $4.65836 \mathrm{E}-04$ & $4.56118 \mathrm{E}-04$ & $4.52418 \mathrm{E}-04$ \\
\hline$T c-107$ & $0.00000 \mathrm{E}+00$ & $0.00000 \mathrm{E}+00$ & $0.00000 \mathrm{E}+00$ & $1.74392 \mathrm{E}-04$ & $1.70320 \mathrm{E}-04$ & $1.69083 \mathrm{E}-04$ \\
\hline$T c-108$ & $0.00000 \mathrm{E}+00$ & $0.00000 \mathrm{E}+00$ & $0.00000 \mathrm{E}+00$ & $2.02398 \mathrm{E}-05$ & $1.97244 \mathrm{E}-05$ & $1.96733 \mathrm{E}-05$ \\
\hline$T c-109$ & $0.00000 \mathrm{E}+00$ & $0.00000 \mathrm{E}+00$ & $0.00000 \mathrm{E}+00$ & $1.57546 \mathrm{E}-06$ & $1.53536 \mathrm{E}-06$ & $1.53060 \mathrm{E}-06$ \\
\hline Tc-110 & $0.00000 \mathrm{E}+00$ & $0.00000 \mathrm{E}+00$ & $0.00000 \mathrm{E}+00$ & $3.15000 \mathrm{E}-07$ & $3.08514 \mathrm{E}-07$ & $3.09390 \mathrm{E}-07$ \\
\hline$T c-111$ & $0.00000 \mathrm{E}+00$ & $0.00000 \mathrm{E}+00$ & $0.00000 \mathrm{E}+00$ & $1.85884 \mathrm{E}-08$ & $1.83420 \mathrm{E}-08$ & $1.83940 \mathrm{E}-08$ \\
\hline$T c-112$ & $0.00000 \mathrm{E}+00$ & $0.00000 \mathrm{E}+00$ & $0.00000 \mathrm{E}+00$ & $2.24570 \mathrm{E}-09$ & $2.24772 \mathrm{E}-09$ & $2.25237 \mathrm{E}-09$ \\
\hline
\end{tabular}




\begin{tabular}{|c|c|c|c|c|c|c|}
\hline \multirow[b]{2}{*}{ Nuclide } & \multicolumn{3}{|c|}{ Fresh Fuel } & \multicolumn{3}{|c|}{ Discharge } \\
\hline & Unit Cell & $\begin{array}{l}\text { Full Core } \\
\text { (NO LBP) }\end{array}$ & Full Core (3) & Unit Cell & $\begin{array}{l}\text { Full Core } \\
\text { (NO LBP) }\end{array}$ & Full Core (3) \\
\hline Tc-113 & $0.00000 \mathrm{E}+00$ & $0.00000 \mathrm{E}+00$ & $0.00000 \mathrm{E}+00$ & $2.20070 \mathrm{E}-10$ & $2.23736 \mathrm{E}-10$ & $2.23979 \mathrm{E}-10$ \\
\hline Tc-114 & $0.00000 \mathrm{E}+00$ & $0.00000 \mathrm{E}+00$ & $0.00000 \mathrm{E}+00$ & $8.73326 \mathrm{E}-11$ & $8.67452 \mathrm{E}-11$ & 8.49267E-11 \\
\hline$T c-115$ & $0.00000 \mathrm{E}+00$ & $0.00000 \mathrm{E}+00$ & $0.00000 \mathrm{E}+00$ & $2.13805 \mathrm{E}-11$ & $2.08574 \mathrm{E}-11$ & $2.10395 \mathrm{E}-11$ \\
\hline$T c-116$ & $0.00000 \mathrm{E}+00$ & $0.00000 \mathrm{E}+00$ & $0.00000 \mathrm{E}+00$ & $3.12527 \mathrm{E}-13$ & $3.13407 \mathrm{E}-13$ & $3.14182 \mathrm{E}-13$ \\
\hline Tc-117 & $0.00000 \mathrm{E}+00$ & $0.00000 \mathrm{E}+00$ & $0.00000 \mathrm{E}+00$ & $4.27668 \mathrm{E}-15$ & 4.37746E-15 & 4.38424E-15 \\
\hline Tc-118 & $0.00000 \mathrm{E}+00$ & $0.00000 \mathrm{E}+00$ & $0.00000 \mathrm{E}+00$ & $9.20976 \mathrm{E}-17$ & $1.12600 \mathrm{E}-16$ & $1.09525 \mathrm{E}-16$ \\
\hline$R u-95$ & $0.00000 \mathrm{E}+00$ & $0.00000 \mathrm{E}+00$ & $0.00000 \mathrm{E}+00$ & $0.00000 \mathrm{E}+00$ & $0.00000 \mathrm{E}+00$ & $0.00000 \mathrm{E}+00$ \\
\hline$R u-97$ & $0.00000 \mathrm{E}+00$ & $0.00000 \mathrm{E}+00$ & $0.00000 \mathrm{E}+00$ & $3.37412 \mathrm{E}-15$ & 3.49794E-15 & $3.73653 \mathrm{E}-15$ \\
\hline$R u-107$ & $0.00000 \mathrm{E}+00$ & $0.00000 \mathrm{E}+00$ & $0.00000 \mathrm{E}+00$ & $2.23054 \mathrm{E}-03$ & $2.17878 \mathrm{E}-03$ & $44 \mathrm{E}-03$ \\
\hline$R u-108$ & $0.00000 \mathrm{E}+00$ & $0.00000 \mathrm{E}+00$ & $0.00000 \mathrm{E}+00$ & $1.84941 \mathrm{E}-03$ & $1.80336 \mathrm{E}-03$ & $1.78808 \mathrm{E}-03$ \\
\hline$R u-109$ & $0.00000 \mathrm{E}+00$ & $0.00000 \mathrm{E}+00$ & $0.00000 \mathrm{E}+00$ & & $1.06844 \mathrm{E}-04$ & $5 \mathrm{E}-04$ \\
\hline$R u-110$ & $0.00000 \mathrm{E}+00$ & $0.00000 \mathrm{E}+00$ & $0.00000 \mathrm{E}+00$ & $7412 \mathrm{E}-05$ & $2.51081 \mathrm{E}-05$ & $2.49432 \mathrm{E}-05$ \\
\hline$R u-111$ & $0.00000 \mathrm{E}+00$ & $0.00000 \mathrm{E}+00$ & $0.00000 \mathrm{E}+00$ & & $1.97768 \mathrm{E}-06$ & $1.96596 \mathrm{E}-06$ \\
\hline$R u-112$ & $0.00000 \mathrm{E}+00$ & $0.00000 \mathrm{E}+00$ & $0.00000 \mathrm{E}+00$ & & $6.13876 \mathrm{E}-07$ & $6.10120 \mathrm{E}-07$ \\
\hline$R u-113$ & $0.00000 \mathrm{E}+00$ & $0.00000 \mathrm{E}+00$ & $0.00000 \mathrm{E}+00$ & $1.36731 \mathrm{E}-07$ & $1.33917 \mathrm{E}-07$ & $1.33416 \mathrm{E}-07$ \\
\hline$R u-114$ & $0.00000 \mathrm{E}+00$ & $0.00000 \mathrm{E}+00$ & $0.00000 \mathrm{E}+00$ & $3.13137 \mathrm{E}-08$ & $3.07084 \mathrm{E}-08$ & $3.04819 \mathrm{E}-08$ \\
\hline$R u-115$ & $0.00000 \mathrm{E}+00$ & $0.00000 \mathrm{E}+00$ & $0.00000 \mathrm{E}+00$ & 8.76516E-09 & $8.57871 \mathrm{E}-09$ & $8.58019 \mathrm{E}-09$ \\
\hline$R u-116$ & $0.00000 \mathrm{E}+00$ & $0.00000 \mathrm{E}+00$ & $0.00000 \mathrm{E}+00$ & $7.68876 \mathrm{E}-10$ & $7.58670 \mathrm{E}-10$ & $7.58253 \mathrm{E}-10$ \\
\hline$R u-117$ & $0.00000 \mathrm{E}+00$ & $0.00000 \mathrm{E}+00$ & $0.00000 \mathrm{E}+00$ & $2.15066 \mathrm{E}-11$ & $2.16302 \mathrm{E}-11$ & $16159 \mathrm{E}-11$ \\
\hline$R u-118$ & $0.00000 \mathrm{E}+00$ & $0.00000 \mathrm{E}+00$ & $0.00000 \mathrm{E}+00$ & $1.34569 \mathrm{E}-12$ & $1.43562 \mathrm{E}-12$ & $1.42393 \mathrm{E}-12$ \\
\hline$R u-119$ & $0.00000 \mathrm{E}+00$ & $0.00000 \mathrm{E}+00$ & $0.00000 \mathrm{E}+00$ & 2.63452E-14 & $2.95148 \mathrm{E}-14$ & $.91119 \mathrm{E}-14$ \\
\hline$R u-120$ & $0.00000 \mathrm{E}+00$ & $0.00000 \mathrm{E}+00$ & $0.00000 \mathrm{E}+00$ & $8.44852 \mathrm{E}-16$ & $9.64238 \mathrm{E}-16$ & $0 \mathrm{E}-16$ \\
\hline$R h-99$ & $0.00000 \mathrm{E}+00$ & $0.00000 \mathrm{E}+00$ & $0.00000 \mathrm{E}+00$ & $1.54629 \mathrm{E}-15$ & $1.52283 \mathrm{E}-15$ & $32 \mathrm{E}-15$ \\
\hline$R h-99 m$ & $0.00000 \mathrm{E}+00$ & $0.00000 \mathrm{E}+00$ & $0.00000 \mathrm{E}+00$ & $0.00000 \mathrm{E}+00$ & $0.00000 \mathrm{E}+00$ & $0.00000 \mathrm{E}+00$ \\
\hline$R h-101$ & $0.00000 \mathrm{E}+00$ & $0.00000 \mathrm{E}+00$ & $0.00000 \mathrm{E}+00$ & $8.29841 \mathrm{E}-09$ & $8.33215 \mathrm{E}-09$ & 22104E-09 \\
\hline$R h-101 m$ & $0.00000 \mathrm{E}+00$ & $0.00000 \mathrm{E}+00$ & $0.00000 \mathrm{E}+00$ & $2.63271 \mathrm{E}-11$ & $2.58762 \mathrm{E}-11$ & $2.53992 \mathrm{E}-11$ \\
\hline$R h-102$ & $0.00000 \mathrm{E}+00$ & $0.00000 \mathrm{E}+00$ & $0.00000 \mathrm{E}+00$ & $1.81325 \mathrm{E}-04$ & $2.14437 \mathrm{E}-04$ & $2.23402 \mathrm{E}-04$ \\
\hline$R h-102 m$ & $0.00000 \mathrm{E}+00$ & $0.00000 \mathrm{E}+00$ & $0.00000 \mathrm{E}+00$ & $2.21414 \mathrm{E}-07$ & $2.18672 \mathrm{E}-07$ & $2.21712 \mathrm{E}-07$ \\
\hline$R h-103 m$ & $0.00000 \mathrm{E}+00$ & $0.00000 \mathrm{E}+00$ & $0.00000 \mathrm{E}+00$ & & $1.00059 \mathrm{E}-01$ & $9.98292 \mathrm{E}-02$ \\
\hline$R h-104$ & $0.00000 \mathrm{E}+00$ & $0.00000 \mathrm{E}+00$ & $0.00000 \mathrm{E}+00$ & & $8.16557 \mathrm{E}-04$ & $8.02747 \mathrm{E}-04$ \\
\hline$R h-104 m$ & $0.00000 \mathrm{E}+00$ & $0.00000 \mathrm{E}+00$ & $0.00000 \mathrm{E}+00$ & $2.36930 \mathrm{E}-09$ & $2.31956 \mathrm{E}-09$ & $2.26557 \mathrm{E}-09$ \\
\hline$R h-105 m$ & $0.00000 \mathrm{E}+00$ & $0.00000 \mathrm{E}+00$ & $0.00000 \mathrm{E}+00$ & $2.21922 \mathrm{E}-04$ & $2.18286 \mathrm{E}-04$ & $2.16447 \mathrm{E}-04$ \\
\hline$R h-106$ & $0.00000 \mathrm{E}+00$ & $0.00000 \mathrm{E}+00$ & $0.00000 \mathrm{E}+00$ & $3.30807 \mathrm{E}-04$ & $3.23630 \mathrm{E}-04$ & $3.28339 \mathrm{E}-04$ \\
\hline$R h-106 m$ & $0.00000 \mathrm{E}+00$ & $0.00000 \mathrm{E}+00$ & $0.00000 \mathrm{E}+00$ & $3.51476 \mathrm{E}-06$ & $3.45215 \mathrm{E}-06$ & $3.38384 \mathrm{E}-06$ \\
\hline$R h-107$ & $0.00000 \mathrm{E}+00$ & $0.00000 \mathrm{E}+00$ & $0.00000 \mathrm{E}+00$ & $1.30809 \mathrm{E}-02$ & $1.27775 \mathrm{E}-02$ & $1.26558 \mathrm{E}-02$ \\
\hline$R h-108$ & $0.00000 \mathrm{E}+00$ & $0.00000 \mathrm{E}+00$ & $0.00000 \mathrm{E}+00$ & $1.14472 \mathrm{E}-04$ & $1.11624 \mathrm{E}-04$ & $1.10671 \mathrm{E}-04$ \\
\hline
\end{tabular}




\section{Depletion Analysis of Modular High Temperature Gas-cooled Reactor Loaded with LEU/Thorium Fuel}

February 2012

\begin{tabular}{|c|c|c|c|c|c|c|}
\hline \multirow[b]{2}{*}{ Nuclide } & \multicolumn{3}{|c|}{ Fresh Fuel } & \multicolumn{3}{|c|}{ Discharge } \\
\hline & Unit Cell & $\begin{array}{l}\text { Full Core } \\
(N O L B P)\end{array}$ & Full Core (3) & Unit Cell & $\begin{array}{l}\text { Full Core } \\
\text { (NO LBP) }\end{array}$ & Full Core (3) \\
\hline$R h-108 m$ & $0.00000 \mathrm{E}+00$ & $0.00000 \mathrm{E}+00$ & $0.00000 \mathrm{E}+00$ & $1.42279 \mathrm{E}-05$ & $1.39084 \mathrm{E}-05$ & $1.36341 \mathrm{E}-05$ \\
\hline Rh-109 & $0.00000 \mathrm{E}+00$ & $0.00000 \mathrm{E}+00$ & $0.00000 \mathrm{E}+00$ & $2.61020 \mathrm{E}-04$ & $2.54368 \mathrm{E}-04$ & $2.52584 \mathrm{E}-04$ \\
\hline$R h-110$ & $0.00000 \mathrm{E}+00$ & $0.00000 \mathrm{E}+00$ & $0.00000 \mathrm{E}+00$ & $7.21966 \mathrm{E}-06$ & $7.04216 \mathrm{E}-06$ & $6.99526 \mathrm{E}-06$ \\
\hline$R h-110 m$ & $0.00000 \mathrm{E}+00$ & $0.00000 \mathrm{E}+00$ & $0.00000 \mathrm{E}+00$ & $1.05849 \mathrm{E}-06$ & $1.03312 \mathrm{E}-06$ & $1.02033 \mathrm{E}-06$ \\
\hline$R h-111$ & $0.00000 \mathrm{E}+00$ & $0.00000 \mathrm{E}+00$ & $0.00000 \mathrm{E}+00$ & $1.18121 \mathrm{E}-05$ & $1.15344 \mathrm{E}-05$ & $1.14599 \mathrm{E}-05$ \\
\hline Rh-112 & $0.00000 \mathrm{E}+00$ & $0.00000 \mathrm{E}+00$ & $0.00000 \mathrm{E}+00$ & $1.62143 \mathrm{E}-06$ & $1.58820 \mathrm{E}-06$ & $1.57756 \mathrm{E}-06$ \\
\hline$R h-113$ & $0.00000 \mathrm{E}+00$ & $0.00000 \mathrm{E}+00$ & $0.00000 \mathrm{E}+00$ & $9.28486 \mathrm{E}-07$ & $9.11021 \mathrm{E}-07$ & $9.06140 \mathrm{E}-07$ \\
\hline$R h-114$ & $0.00000 \mathrm{E}+00$ & $0.00000 \mathrm{E}+00$ & $0.00000 \mathrm{E}+00$ & $3.29055 \mathrm{E}-07$ & $3.23208 \mathrm{E}-07$ & $.20816 \mathrm{E}-07$ \\
\hline$R h-115$ & $0.00000 \mathrm{E}+00$ & $0.00000 \mathrm{E}+00$ & $0.00000 \mathrm{E}+00$ & $7979 \mathrm{E}-08$ & $7.96043 \mathrm{E}-08$ & $.91190 \mathrm{E}-08$ \\
\hline$R h-116$ & $0.00000 \mathrm{E}+00$ & $0.00000 \mathrm{E}+00$ & $0.00000 \mathrm{E}+00$ & $1.92037 \mathrm{E}-08$ & $1.89044 \mathrm{E}-08$ & 87791E-08 \\
\hline$R h-117$ & $0.00000 \mathrm{E}+00$ & $0.00000 \mathrm{E}+00$ & $0.00000 \mathrm{E}+00$ & 3.49914E-09 & $3.46880 \mathrm{E}-09$ & 45798E-09 \\
\hline$R h-118$ & $0.00000 \mathrm{E}+00$ & $0.00000 \mathrm{E}+00$ & $0.00000 \mathrm{E}+00$ & $3.16366 \mathrm{E}-10$ & $3.17505 \mathrm{E}-10$ & $16874 \mathrm{E}-10$ \\
\hline$R h-119$ & $0.00000 \mathrm{E}+00$ & $0.00000 \mathrm{E}+00$ & $0.00000 \mathrm{E}+00$ & $4.47547 \mathrm{E}-11$ & $4.60305 \mathrm{E}-11$ & 819E-11 \\
\hline$R h-120$ & $0.00000 \mathrm{E}+00$ & $0.00000 \mathrm{E}+00$ & $0.00000 \mathrm{E}+00$ & $3.03876 \mathrm{E}-12$ & $3.13948 \mathrm{E}-12$ & $.14668 \mathrm{E}-12$ \\
\hline$R h-121$ & $0.00000 \mathrm{E}+00$ & $0.00000 \mathrm{E}+00$ & $0.00000 \mathrm{E}+00$ & $8.87246 \mathrm{E}-14$ & $9.84028 \mathrm{E}-14$ & $.71406 \mathrm{E}-14$ \\
\hline$R h-122$ & $0.00000 \mathrm{E}+00$ & $0.00000 \mathrm{E}+00$ & $0.00000 \mathrm{E}+00$ & $7.71073 \mathrm{E}-16$ & $9.02225 \mathrm{E}-16$ & $8.83195 \mathrm{E}-16$ \\
\hline$P d-99$ & $0.00000 \mathrm{E}+00$ & $0.00000 \mathrm{E}+00$ & $0.00000 \mathrm{E}+00$ & $0.00000 \mathrm{E}+00$ & $0.00000 \mathrm{E}+00$ & $0.00000 \mathrm{E}+00$ \\
\hline$P d-101$ & $0.00000 \mathrm{E}+00$ & $0.00000 \mathrm{E}+00$ & $0.00000 \mathrm{E}+00$ & $5.95019 \mathrm{E}-15$ & $8.31072 \mathrm{E}-15$ & $9.27870 \mathrm{E}-15$ \\
\hline$P d-103$ & $0.00000 \mathrm{E}+00$ & $0.00000 \mathrm{E}+00$ & $0.00000 \mathrm{E}+00$ & $6.10117 \mathrm{E}-06$ & $7.02722 \mathrm{E}-06$ & 7.15294E-06 \\
\hline$P d-107 m$ & $0.00000 \mathrm{E}+00$ & $0.00000 \mathrm{E}+00$ & $0.00000 \mathrm{E}+00$ & $4.81461 \mathrm{E}-10$ & $4.71465 \mathrm{E}-10$ & $4.60556 \mathrm{E}-10$ \\
\hline$P d-109$ & $0.00000 \mathrm{E}+00$ & $0.00000 \mathrm{E}+00$ & $0.00000 \mathrm{E}+00$ & $1.95153 \mathrm{E}-01$ & $1.90412 \mathrm{E}-01$ & $1.89984 \mathrm{E}-01$ \\
\hline$P d-109 m$ & $0.00000 \mathrm{E}+00$ & $0.00000 \mathrm{E}+00$ & $0.00000 \mathrm{E}+00$ & $3.13012 \mathrm{E}-08$ & $3.01692 \mathrm{E}-08$ & 3.09292E-08 \\
\hline$P d-111$ & $0.00000 \mathrm{E}+00$ & $0.00000 \mathrm{E}+00$ & $0.00000 \mathrm{E}+00$ & $1.51349 \mathrm{E}-03$ & $1.47828 \mathrm{E}-03$ & $1.46889 \mathrm{E}-03$ \\
\hline$P d-111 m$ & $0.00000 \mathrm{E}+00$ & $0.00000 \mathrm{E}+00$ & $0.00000 \mathrm{E}+00$ & $7.58179 \mathrm{E}-06$ & 7.37847E-06 & 7.83774E-06 \\
\hline$P d-112$ & $0.00000 \mathrm{E}+00$ & $0.00000 \mathrm{E}+00$ & $0.00000 \mathrm{E}+00$ & $3.58220 \mathrm{E}-02$ & $3.50896 \mathrm{E}-02$ & 3.48684E-02 \\
\hline$P d-113$ & $0.00000 \mathrm{E}+00$ & $0.00000 \mathrm{E}+00$ & $0.00000 \mathrm{E}+00$ & $3.19164 \mathrm{E}-05$ & $3.13455 \mathrm{E}-05$ & $.11924 \mathrm{E}-05$ \\
\hline$P d-114$ & $0.00000 \mathrm{E}+00$ & $0.00000 \mathrm{E}+00$ & $0.00000 \mathrm{E}+00$ & $3.32699 \mathrm{E}-05$ & $3.27972 \mathrm{E}-05$ & $3.25554 \mathrm{E}-05$ \\
\hline$P d-115$ & $0.00000 \mathrm{E}+00$ & $0.00000 \mathrm{E}+00$ & $0.00000 \mathrm{E}+00$ & 4.25294E-06 & 4.21307E-06 & 4.18313E-06 \\
\hline$P d-116$ & $0.00000 \mathrm{E}+00$ & $0.00000 \mathrm{E}+00$ & $0.00000 \mathrm{E}+00$ & $1.75262 \mathrm{E}-06$ & $1.73436 \mathrm{E}-06$ & $1.71643 \mathrm{E}-06$ \\
\hline$P d-117$ & $0.00000 \mathrm{E}+00$ & $0.00000 \mathrm{E}+00$ & $0.00000 \mathrm{E}+00$ & $4.62351 \mathrm{E}-07$ & $4.58937 \mathrm{E}-07$ & $.56383 \mathrm{E}-07$ \\
\hline$P d-118$ & $0.00000 \mathrm{E}+00$ & $0.00000 \mathrm{E}+00$ & $0.00000 \mathrm{E}+00$ & $8.46589 \mathrm{E}-08$ & $8.41084 \mathrm{E}-08$ & 8.37103E-08 \\
\hline$P d-119$ & $0.00000 \mathrm{E}+00$ & $0.00000 \mathrm{E}+00$ & $0.00000 \mathrm{E}+00$ & $9.57752 \mathrm{E}-09$ & $9.52743 \mathrm{E}-09$ & 9.49581E-09 \\
\hline$P d-120$ & $0.00000 \mathrm{E}+00$ & $0.00000 \mathrm{E}+00$ & $0.00000 \mathrm{E}+00$ & $6.59010 \mathrm{E}-09$ & $6.61270 \mathrm{E}-09$ & $6.67235 \mathrm{E}-09$ \\
\hline$P d-121$ & $0.00000 \mathrm{E}+00$ & $0.00000 \mathrm{E}+00$ & $0.00000 \mathrm{E}+00$ & $1.87962 \mathrm{E}-10$ & $1.90584 \mathrm{E}-10$ & $1.90964 \mathrm{E}-10$ \\
\hline$P d-122$ & $0.00000 \mathrm{E}+00$ & $0.00000 \mathrm{E}+00$ & $0.00000 \mathrm{E}+00$ & $2.37240 \mathrm{E}-11$ & $2.46225 \mathrm{E}-11$ & $2.46003 \mathrm{E}-11$ \\
\hline$P d-123$ & $0.00000 \mathrm{E}+00$ & $0.00000 \mathrm{E}+00$ & $0.00000 \mathrm{E}+00$ & $8.14824 \mathrm{E}-13$ & $8.70872 \mathrm{E}-13$ & $8.67462 \mathrm{E}-13$ \\
\hline
\end{tabular}




\begin{tabular}{|c|c|c|c|c|c|c|}
\hline \multirow[b]{2}{*}{ Nuclide } & \multicolumn{3}{|c|}{ Fresh Fuel } & \multicolumn{3}{|c|}{ Discharge } \\
\hline & Unit Cell & $\begin{array}{l}\text { Full Core } \\
\text { (NO LBP) }\end{array}$ & Full Core (3) & Unit Cell & $\begin{array}{l}\text { Full Core } \\
\text { (NO LBP) }\end{array}$ & Full Core (3) \\
\hline$P d-124$ & $0.00000 \mathrm{E}+00$ & $0.00000 \mathrm{E}+00$ & $0.00000 \mathrm{E}+00$ & $2.54314 \mathrm{E}-13$ & $2.58803 \mathrm{E}-13$ & $2.60894 \mathrm{E}-13$ \\
\hline Ag-103 & $0.00000 \mathrm{E}+00$ & $0.00000 \mathrm{E}+00$ & $0.00000 \mathrm{E}+00$ & $0.00000 \mathrm{E}+00$ & $0.00000 \mathrm{E}+00$ & $0.00000 \mathrm{E}+00$ \\
\hline Ag-104 & $0.00000 \mathrm{E}+00$ & $0.00000 \mathrm{E}+00$ & $0.00000 \mathrm{E}+00$ & $1.73998 \mathrm{E}-28$ & $2.08830 \mathrm{E}-28$ & $2.20493 \mathrm{E}-28$ \\
\hline Ag-105 & $0.00000 \mathrm{E}+00$ & $0.00000 \mathrm{E}+00$ & $0.00000 \mathrm{E}+00$ & $1.66776 \mathrm{E}-13$ & $1.63174 \mathrm{E}-13$ & $1.76466 \mathrm{E}-13$ \\
\hline $\mathrm{Ag}-105 \mathrm{~m}$ & $0.00000 \mathrm{E}+00$ & $0.00000 \mathrm{E}+00$ & $0.00000 \mathrm{E}+00$ & $2.09245 \mathrm{E}-17$ & $2.05176 \mathrm{E}-17$ & $2.20052 \mathrm{E}-17$ \\
\hline Ag-106 & $0.00000 \mathrm{E}+00$ & $0.00000 \mathrm{E}+00$ & $0.00000 \mathrm{E}+00$ & $1.07717 \mathrm{E}-14$ & $1.07448 \mathrm{E}-14$ & $7246 \mathrm{E}-14$ \\
\hline Ag- $106 \mathrm{~m}$ & $0.00000 \mathrm{E}+00$ & $0.00000 \mathrm{E}+00$ & $0.00000 \mathrm{E}+00$ & $1.14824 \mathrm{E}-11$ & $1.12410 \mathrm{E}-11$ & $77 \mathrm{E}-11$ \\
\hline Ag- $107 \mathrm{~m}$ & $0.00000 \mathrm{E}+00$ & $0.00000 \mathrm{E}+00$ & $0.00000 \mathrm{E}+00$ & $6.96998 \mathrm{E}-14$ & $6.82624 \mathrm{E}-14$ & $22 \mathrm{E}-14$ \\
\hline Ag-108 & $0.00000 \mathrm{E}+00$ & $0.00000 \mathrm{E}+00$ & $0.00000 \mathrm{E}+00$ & $2.29672 \mathrm{E}-10$ & $2.66856 \mathrm{E}-10$ & $5 \mathrm{E}-10$ \\
\hline Ag-108m & $0.00000 \mathrm{E}+00$ & $0.00000 \mathrm{E}+00$ & $0.00000 \mathrm{E}+00$ & $1.50885 \mathrm{E}-06$ & $1.45750 \mathrm{E}-06$ & $9 \mathrm{E}-06$ \\
\hline $\mathrm{Ag}-109 \mathrm{~m}$ & $0.00000 \mathrm{E}+00$ & $0.00000 \mathrm{E}+00$ & $0000 \mathrm{E}+00$ & & $1.52729 \mathrm{E}-04$ & 7E-04 \\
\hline Ag- 110 & $0.00000 \mathrm{E}+00$ & $0.00000 \mathrm{E}+00$ & $0.00000 \mathrm{E}+00$ & $0 \mathrm{E}-06$ & $2.54018 \mathrm{E}-06$ & $77 \mathrm{E}-06$ \\
\hline $\mathrm{Ag}-111 \mathrm{~m}$ & $0.00000 \mathrm{E}+00$ & $0.00000 \mathrm{E}+00$ & $0.00000 \mathrm{E}+00$ & $6.77811 \mathrm{E}-05$ & $6.62042 \mathrm{E}-05$ & $6.57836 \mathrm{E}-05$ \\
\hline Ag-112 & $0.00000 \mathrm{E}+00$ & $0.00000 \mathrm{E}+00$ & $0.00000 \mathrm{E}+00$ & $5.34262 \mathrm{E}-03$ & $5.23371 \mathrm{E}-03$ & $5.20060 \mathrm{E}-03$ \\
\hline Ag-113 & $0.00000 \mathrm{E}+00$ & $0.00000 \mathrm{E}+00$ & $0.00000 \mathrm{E}+00$ & $4.35355 \mathrm{E}-03$ & $4.27566 \mathrm{E}-03$ & $4.25502 \mathrm{E}-03$ \\
\hline Ag-113m & $0.00000 \mathrm{E}+00$ & $0.00000 \mathrm{E}+00$ & $0.00000 \mathrm{E}+00$ & $2.25501 \mathrm{E}-05$ & $2.21468 \mathrm{E}-05$ & $2.20392 \mathrm{E}-05$ \\
\hline Ag-114 & $0.00000 \mathrm{E}+00$ & $0.00000 \mathrm{E}+00$ & $0.00000 \mathrm{E}+00$ & $1.07413 \mathrm{E}-06$ & $1.05924 \mathrm{E}-06$ & $1.05172 \mathrm{E}-06$ \\
\hline Ag-115 & $0.00000 \mathrm{E}+00$ & $0.00000 \mathrm{E}+00$ & $0.00000 \mathrm{E}+00$ & $2.10902 \mathrm{E}-04$ & $2.09028 \mathrm{E}-04$ & 3E-04 \\
\hline Ag-115m & $0.00000 \mathrm{E}+00$ & $0.00000 \mathrm{E}+00$ & $0.00000 \mathrm{E}+00$ & $1.54157 \mathrm{E}-07$ & $1.54877 \mathrm{E}-07$ & $4 \mathrm{E}-07$ \\
\hline Ag-116 & $0.00000 \mathrm{E}+00$ & $0.00000 \mathrm{E}+00$ & $0.00000 \mathrm{E}+00$ & $2.86608 \mathrm{E}-05$ & $2.84075 \mathrm{E}-05$ & $0 \mathrm{E}-05$ \\
\hline $\mathrm{Ag}-116 \mathrm{~m}$ & $0.00000 \mathrm{E}+00$ & $0.00000 \mathrm{E}+00$ & $0.00000 \mathrm{E}+00$ & $1.10392 \mathrm{E}-07$ & $1.09740 \mathrm{E}-07$ & $08370 \mathrm{E}-07$ \\
\hline Ag-117 & $0.00000 \mathrm{E}+00$ & $0.00000 \mathrm{E}+00$ & $0.00000 \mathrm{E}+00$ & $1.02939 \mathrm{E}-05$ & $1.02184 \mathrm{E}-05$ & $3 \mathrm{E}-05$ \\
\hline $\mathrm{Ag}-117 \mathrm{~m}$ & $0.00000 \mathrm{E}+00$ & $0.00000 \mathrm{E}+00$ & $0.00000 \mathrm{E}+00$ & $1.64382 \mathrm{E}-07$ & $1.63203 \mathrm{E}-07$ & E-07 \\
\hline Ag-118 & $0.00000 \mathrm{E}+00$ & $0.00000 \mathrm{E}+00$ & $0.00000 \mathrm{E}+00$ & $3.87408 \mathrm{E}-07$ & $3.84831 \mathrm{E}-07$ & $9 \mathrm{E}-07$ \\
\hline Ag-118m & $0.00000 \mathrm{E}+00$ & $0.00000 \mathrm{E}+00$ & $0.00000 \mathrm{E}+00$ & $4774 \mathrm{E}-08$ & $7.99079 \mathrm{E}-08$ & $2410 \mathrm{E}-08$ \\
\hline Ag-119 & $0.00000 \mathrm{E}+00$ & $0.00000 \mathrm{E}+00$ & $0.00000 \mathrm{E}+00$ & $1.86138 \mathrm{E}-07$ & $1.85145 \mathrm{E}-07$ & $4392 \mathrm{E}-07$ \\
\hline Ag-120 & $0.00000 \mathrm{E}+00$ & $0.00000 \mathrm{E}+00$ & $0.00000 \mathrm{E}+00$ & 4.00144E-08 & $3.98306 \mathrm{E}-08$ & $98285 \mathrm{E}-08$ \\
\hline $\mathrm{Ag}-120 \mathrm{~m}$ & $0.00000 \mathrm{E}+00$ & $0.00000 \mathrm{E}+00$ & $0.00000 \mathrm{E}+00$ & $5.65037 \mathrm{E}-09$ & $5.59351 \mathrm{E}-09$ & $5.55821 \mathrm{E}-09$ \\
\hline Ag-121 & $0.00000 \mathrm{E}+00$ & $0.00000 \mathrm{E}+00$ & $0.00000 \mathrm{E}+00$ & $1.75629 \mathrm{E}-08$ & $1.75176 \mathrm{E}-08$ & $1.75420 \mathrm{E}-08$ \\
\hline Ag-122 & $0.00000 \mathrm{E}+00$ & $0.00000 \mathrm{E}+00$ & $0.00000 \mathrm{E}+00$ & $1.37376 \mathrm{E}-09$ & $1.37646 \mathrm{E}-09$ & $1.37960 \mathrm{E}-09$ \\
\hline $\mathrm{Ag}-122 \mathrm{~m}$ & $0.00000 \mathrm{E}+00$ & $0.00000 \mathrm{E}+00$ & $0.00000 \mathrm{E}+00$ & $4.22722 \mathrm{E}-09$ & $4.23315 \mathrm{E}-09$ & 4.24305E-09 \\
\hline Ag-123 & $0.00000 \mathrm{E}+00$ & $0.00000 \mathrm{E}+00$ & $0.00000 \mathrm{E}+00$ & $4.85490 \mathrm{E}-10$ & $4.90111 \mathrm{E}-10$ & $4.93242 \mathrm{E}-10$ \\
\hline Ag-124 & $0.00000 \mathrm{E}+00$ & $0.00000 \mathrm{E}+00$ & $0.00000 \mathrm{E}+00$ & $4.18412 \mathrm{E}-10$ & $4.20451 \mathrm{E}-10$ & $4.24978 \mathrm{E}-10$ \\
\hline Ag-125 & $0.00000 \mathrm{E}+00$ & $0.00000 \mathrm{E}+00$ & $0.00000 \mathrm{E}+00$ & $5.52700 \mathrm{E}-13$ & $6.84288 \mathrm{E}-13$ & $6.64030 \mathrm{E}-13$ \\
\hline Ag-126 & $0.00000 \mathrm{E}+00$ & $0.00000 \mathrm{E}+00$ & $0.00000 \mathrm{E}+00$ & $2.72264 \mathrm{E}-16$ & $2.77544 \mathrm{E}-16$ & $2.79331 \mathrm{E}-16$ \\
\hline Ag-127 & $0.00000 \mathrm{E}+00$ & $0.00000 \mathrm{E}+00$ & $0.00000 \mathrm{E}+00$ & $5.90022 \mathrm{E}-17$ & $5.96274 \mathrm{E}-17$ & $6.01790 \mathrm{E}-17$ \\
\hline
\end{tabular}




\section{Depletion Analysis of Modular High Temperature Gas-cooled Reactor Loaded with LEU/Thorium Fuel}

February 2012

\begin{tabular}{|c|c|c|c|c|c|c|}
\hline \multirow[b]{2}{*}{ Nuclide } & \multicolumn{3}{|c|}{ Fresh Fuel } & \multicolumn{3}{|c|}{ Discharge } \\
\hline & Unit Cell & $\begin{array}{l}\text { Full Core } \\
\text { (NO LBP) }\end{array}$ & Full Core (3) & Unit Cell & $\begin{array}{l}\text { Full Core } \\
\text { (NO LBP) }\end{array}$ & Full Core (3) \\
\hline Ag- 128 & $0.00000 \mathrm{E}+00$ & $0.00000 \mathrm{E}+00$ & $0.00000 \mathrm{E}+00$ & $2.98613 \mathrm{E}-18$ & $3.01525 \mathrm{E}-18$ & $3.04482 \mathrm{E}-18$ \\
\hline Ag-129 & $0.00000 \mathrm{E}+00$ & $0.00000 \mathrm{E}+00$ & $0.00000 \mathrm{E}+00$ & 3.89844E-14 & 4.00494E-14 & $3.95600 \mathrm{E}-14$ \\
\hline Ag-130 & $0.00000 \mathrm{E}+00$ & $0.00000 \mathrm{E}+00$ & $0.00000 \mathrm{E}+00$ & $1.51374 \mathrm{E}-12$ & $1.51860 \mathrm{E}-12$ & $1.53552 \mathrm{E}-12$ \\
\hline $\mathrm{Cd}-105$ & $0.00000 \mathrm{E}+00$ & $0.00000 \mathrm{E}+00$ & $0.00000 \mathrm{E}+00$ & $2.64463 \mathrm{E}-23$ & $2.99374 \mathrm{E}-23$ & $3.26732 \mathrm{E}-23$ \\
\hline$C d-107$ & $0.00000 \mathrm{E}+00$ & $0.00000 \mathrm{E}+00$ & $0.00000 \mathrm{E}+00$ & $3.63797 \mathrm{E}-15$ & $4.79285 \mathrm{E}-15$ & $5.49178 \mathrm{E}-15$ \\
\hline Cd-109 & $0.00000 \mathrm{E}+00$ & $0.00000 \mathrm{E}+00$ & $0.00000 \mathrm{E}+00$ & $1.27809 \mathrm{E}-06$ & $1.40195 \mathrm{E}-06$ & $1.61306 \mathrm{E}-06$ \\
\hline $\mathrm{Cd}-111 \mathrm{~m}$ & $0.00000 \mathrm{E}+00$ & $0.00000 \mathrm{E}+00$ & $0.00000 \mathrm{E}+00$ & $2.39355 \mathrm{E}-11$ & $2.30550 \mathrm{E}-11$ & $2.49816 \mathrm{E}-11$ \\
\hline$C d-113 m$ & $0.00000 \mathrm{E}+00$ & $0.00000 \mathrm{E}+00$ & $0.00000 \mathrm{E}+00$ & $1.54575 \mathrm{E}-01$ & $1.51454 \mathrm{E}-01$ & $56165 \mathrm{E}-01$ \\
\hline$C d-115$ & $0.00000 \mathrm{E}+00$ & $0.00000 \mathrm{E}+00$ & $0.00000 \mathrm{E}+00$ & $3.40219 \mathrm{E}-02$ & $3.37722 \mathrm{E}-02$ & $5505 \mathrm{E}-02$ \\
\hline$C d-117$ & $0.00000 \mathrm{E}+00$ & $0.00000 \mathrm{E}+00$ & $0.00000 \mathrm{E}+00$ & $1.53457 \mathrm{E}-03$ & $1.52366 \mathrm{E}-03$ & $51085 \mathrm{E}-03$ \\
\hline$C d-117 m$ & $0.00000 \mathrm{E}+00$ & $0.00000 \mathrm{E}+00$ & $0.00000 \mathrm{E}+00$ & $7.53468 \mathrm{E}-05$ & $7.53929 \mathrm{E}-05$ & $45050 \mathrm{E}-05$ \\
\hline $\mathrm{Cd}-118$ & $0.00000 \mathrm{E}+00$ & $0.00000 \mathrm{E}+00$ & $0.00000 \mathrm{E}+00$ & & $4.53282 \mathrm{E}-04$ & $0074 \mathrm{E}-04$ \\
\hline$C d-119$ & $0.00000 \mathrm{E}+00$ & $0.00000 \mathrm{E}+00$ & $0.00000 \mathrm{E}+00$ & & $1.98540 \mathrm{E}-05$ & $38 \mathrm{E}-05$ \\
\hline$C d-119 m$ & $0.00000 \mathrm{E}+00$ & $0.00000 \mathrm{E}+00$ & $0.00000 \mathrm{E}+00$ & 4.49858E-06 & 4.49144E-06 & 4.44737E-06 \\
\hline$C d-120$ & $0.00000 \mathrm{E}+00$ & $0.00000 \mathrm{E}+00$ & $0.00000 \mathrm{E}+00$ & 493E-06 & $7.73200 \mathrm{E}-06$ & $7.68811 \mathrm{E}-06$ \\
\hline$C d-121$ & $0.00000 \mathrm{E}+00$ & $0.00000 \mathrm{E}+00$ & $0.00000 \mathrm{E}+00$ & 1014E-06 & $1.00598 \mathrm{E}-06$ & $1.00083 \mathrm{E}-06$ \\
\hline $\mathrm{Cd}-121 \mathrm{~m}$ & $0.00000 \mathrm{E}+00$ & $0.00000 \mathrm{E}+00$ & $0.00000 \mathrm{E}+00$ & $4.72077 \mathrm{E}-07$ & $4.69900 \mathrm{E}-07$ & $.66521 \mathrm{E}-07$ \\
\hline$C d-122$ & $0.00000 \mathrm{E}+00$ & $0.00000 \mathrm{E}+00$ & $0.00000 \mathrm{E}+00$ & $6.58810 \mathrm{E}-07$ & $6.58221 \mathrm{E}-07$ & $6.56314 \mathrm{E}-07$ \\
\hline$C d-123$ & $0.00000 \mathrm{E}+00$ & $0.00000 \mathrm{E}+00$ & $0.00000 \mathrm{E}+00$ & $1.40831 \mathrm{E}-07$ & $1.40783 \mathrm{E}-07$ & $1.41373 \mathrm{E}-07$ \\
\hline$C d-123 m$ & $0.00000 \mathrm{E}+00$ & $0.00000 \mathrm{E}+00$ & $0.00000 \mathrm{E}+00$ & $3.69271 \mathrm{E}-10$ & $3.72786 \mathrm{E}-10$ & $3.75167 \mathrm{E}-10$ \\
\hline$C d-124$ & $0.00000 \mathrm{E}+00$ & $0.00000 \mathrm{E}+00$ & $0.00000 \mathrm{E}+00$ & $7.56612 \mathrm{E}-08$ & $7.59775 \mathrm{E}-08$ & 7.67251E-08 \\
\hline$C d-125$ & $0.00000 \mathrm{E}+00$ & $0.00000 \mathrm{E}+00$ & $0.00000 \mathrm{E}+00$ & $1.64216 \mathrm{E}-08$ & $1.65044 \mathrm{E}-08$ & $1.66722 \mathrm{E}-08$ \\
\hline$C d-126$ & $0.00000 \mathrm{E}+00$ & $0.00000 \mathrm{E}+00$ & $0.00000 \mathrm{E}+00$ & $1.84795 \mathrm{E}-08$ & $1.85410 \mathrm{E}-08$ & $1.87449 \mathrm{E}-08$ \\
\hline$C d-127$ & $0.00000 \mathrm{E}+00$ & $0.00000 \mathrm{E}+00$ & $0.00000 \mathrm{E}+00$ & $1.34212 \mathrm{E}-08$ & $1.34649 \mathrm{E}-08$ & $1.36147 \mathrm{E}-08$ \\
\hline$C d-128$ & $0.00000 \mathrm{E}+00$ & $0.00000 \mathrm{E}+00$ & $0.00000 \mathrm{E}+00$ & 4.45194E-09 & 4.46639E-09 & $4.51618 \mathrm{E}-09$ \\
\hline$C d-129$ & $0.00000 \mathrm{E}+00$ & $0.00000 \mathrm{E}+00$ & $0.00000 \mathrm{E}+00$ & $3.69703 \mathrm{E}-10$ & $3.79843 \mathrm{E}-10$ & $3.75211 \mathrm{E}-10$ \\
\hline$C d-130$ & $0.00000 \mathrm{E}+00$ & $0.00000 \mathrm{E}+00$ & $0.00000 \mathrm{E}+00$ & $6.46363 \mathrm{E}-08$ & $6.48440 \mathrm{E}-08$ & $6.55665 \mathrm{E}-08$ \\
\hline$C d-131$ & $0.00000 \mathrm{E}+00$ & $0.00000 \mathrm{E}+00$ & $0.00000 \mathrm{E}+00$ & $4.29079 \mathrm{E}-09$ & $4.30450 \mathrm{E}-09$ & $4.35258 \mathrm{E}-09$ \\
\hline Cd-132 & $0.00000 \mathrm{E}+00$ & $0.00000 \mathrm{E}+00$ & $0.00000 \mathrm{E}+00$ & $6.68813 \mathrm{E}-13$ & $7.47598 \mathrm{E}-13$ & $7.33228 \mathrm{E}-13$ \\
\hline In-107 & $0.00000 \mathrm{E}+00$ & $0.00000 \mathrm{E}+00$ & $0.00000 \mathrm{E}+00$ & $0.00000 \mathrm{E}+00$ & $0.00000 \mathrm{E}+00$ & $0.00000 \mathrm{E}+00$ \\
\hline In-109 & $0.00000 \mathrm{E}+00$ & $0.00000 \mathrm{E}+00$ & $0.00000 \mathrm{E}+00$ & $7.21736 \mathrm{E}-18$ & $7.07038 \mathrm{E}-18$ & 7.58724E-18 \\
\hline In- 110 & $0.00000 \mathrm{E}+00$ & $0.00000 \mathrm{E}+00$ & $0.00000 \mathrm{E}+00$ & $3.61862 \mathrm{E}-26$ & $5.57009 \mathrm{E}-26$ & $6.01085 \mathrm{E}-26$ \\
\hline In-111 & $0.00000 \mathrm{E}+00$ & $0.00000 \mathrm{E}+00$ & $0.00000 \mathrm{E}+00$ & $6.97549 \mathrm{E}-13$ & $6.80550 \mathrm{E}-13$ & $7.31366 \mathrm{E}-13$ \\
\hline$I n-111 m$ & $0.00000 \mathrm{E}+00$ & $0.00000 \mathrm{E}+00$ & $0.00000 \mathrm{E}+00$ & $3.20047 \mathrm{E}-23$ & $3.20372 \mathrm{E}-23$ & $3.44073 \mathrm{E}-23$ \\
\hline In-112 & $0.00000 \mathrm{E}+00$ & $0.00000 \mathrm{E}+00$ & $0.00000 \mathrm{E}+00$ & $9.44115 \mathrm{E}-14$ & $1.09477 \mathrm{E}-13$ & $1.15806 \mathrm{E}-13$ \\
\hline In-112m & $0.00000 \mathrm{E}+00$ & $0.00000 \mathrm{E}+00$ & $0.00000 \mathrm{E}+00$ & $8.06377 \mathrm{E}-15$ & $7.58517 \mathrm{E}-15$ & $8.45571 \mathrm{E}-15$ \\
\hline
\end{tabular}




\begin{tabular}{|c|c|c|c|c|c|c|}
\hline \multirow[b]{2}{*}{ Nuclide } & \multicolumn{3}{|c|}{ Fresh Fuel } & \multicolumn{3}{|c|}{ Discharge } \\
\hline & Unit Cell & $\begin{array}{l}\text { Full Core } \\
\text { (NO LBP) }\end{array}$ & Full Core (3) & Unit Cell & $\begin{array}{l}\text { Full Core } \\
(N O L B P)\end{array}$ & Full Core (3) \\
\hline$I n-113 m$ & $0.00000 \mathrm{E}+00$ & $0.00000 \mathrm{E}+00$ & $0.00000 \mathrm{E}+00$ & $1.31643 \mathrm{E}-13$ & $1.16881 \mathrm{E}-13$ & $1.42321 \mathrm{E}-13$ \\
\hline $\operatorname{In}-114$ & $0.00000 \mathrm{E}+00$ & $0.00000 \mathrm{E}+00$ & $0.00000 \mathrm{E}+00$ & $2.25730 \mathrm{E}-09$ & $2.21512 \mathrm{E}-09$ & $2.23646 \mathrm{E}-09$ \\
\hline In-114m & $0.00000 \mathrm{E}+00$ & $0.00000 \mathrm{E}+00$ & $0.00000 \mathrm{E}+00$ & $1.42688 \mathrm{E}-09$ & $1.46930 \mathrm{E}-09$ & $1.46153 \mathrm{E}-09$ \\
\hline In-115m & $0.00000 \mathrm{E}+00$ & $0.00000 \mathrm{E}+00$ & $0.00000 \mathrm{E}+00$ & $2.85533 \mathrm{E}-03$ & $2.83437 \mathrm{E}-03$ & $2.81581 \mathrm{E}-03$ \\
\hline In-116 & $0.00000 \mathrm{E}+00$ & $0.00000 \mathrm{E}+00$ & $0.00000 \mathrm{E}+00$ & $2.29840 \mathrm{E}-06$ & $2.27202 \mathrm{E}-06$ & $2.24948 \mathrm{E}-06$ \\
\hline$I n-116 m$ & $0.00000 \mathrm{E}+00$ & $0.00000 \mathrm{E}+00$ & $0.00000 \mathrm{E}+00$ & 2.39322E-09 & $2.42309 \mathrm{E}-09$ & $2.38590 \mathrm{E}-09$ \\
\hline In-116m & $0.00000 \mathrm{E}+00$ & $0.00000 \mathrm{E}+00$ & $0.00000 \mathrm{E}+00$ & 2.39322E-09 & $2.42309 \mathrm{E}-09$ & $2.38590 \mathrm{E}-09$ \\
\hline In-117 & $0.00000 \mathrm{E}+00$ & $0.00000 \mathrm{E}+00$ & $0.00000 \mathrm{E}+00$ & $2.77310 \mathrm{E}-04$ & $2.75464 \mathrm{E}-04$ & $2.73095 \mathrm{E}-04$ \\
\hline$I n-117 m$ & $0.00000 \mathrm{E}+00$ & $0.00000 \mathrm{E}+00$ & $0.00000 \mathrm{E}+00$ & $9.28879 \mathrm{E}-04$ & $9.22279 \mathrm{E}-04$ & $30 \mathrm{E}-04$ \\
\hline In-118 & $0.00000 \mathrm{E}+00$ & $0.00000 \mathrm{E}+00$ & $0.00000 \mathrm{E}+00$ & $7.55861 \mathrm{E}-07$ & $7.52373 \mathrm{E}-07$ & 37E-07 \\
\hline In-118m & $0.00000 \mathrm{E}+00$ & $0.00000 \mathrm{E}+00$ & $0.00000 \mathrm{E}+00$ & $57 \mathrm{E}-08$ & $1.07244 \mathrm{E}-08$ & $8 \mathrm{E}-08$ \\
\hline In-118m & $0.00000 \mathrm{E}+00$ & $0.00000 \mathrm{E}+00$ & $0.00000 \mathrm{E}+00$ & $1.06657 \mathrm{E}-08$ & $1.07244 \mathrm{E}-08$ & $1.05588 \mathrm{E}-08$ \\
\hline In-119 & $0.00000 \mathrm{E}+00$ & $0.00000 \mathrm{E}+00$ & $0.00000 \mathrm{E}+00$ & $1.24219 \mathrm{E}-05$ & $1.23858 \mathrm{E}-05$ & $1.22963 \mathrm{E}-05$ \\
\hline In-119m & & $0.00000 \mathrm{E}+00$ & $0.00000 \mathrm{E}+00$ & $8.72577 \mathrm{E}-05$ & $8.68902 \mathrm{E}-05$ & $8.64005 \mathrm{E}-05$ \\
\hline In-120 & $0.00000 \mathrm{E}+00$ & $0.00000 \mathrm{E}+00$ & $0.00000 \mathrm{E}+00$ & $4.83539 \mathrm{E}-07$ & $4.82132 \mathrm{E}-07$ & 4.79306E-07 \\
\hline In-120m & $0.00000 \mathrm{E}+00$ & $0.00000 \mathrm{E}+00$ & $0.00000 \mathrm{E}+00$ & $2.05294 \mathrm{E}-07$ & $2.05002 \mathrm{E}-07$ & $2.02467 \mathrm{E}-07$ \\
\hline In-120m & $0.00000 \mathrm{E}+00$ & $0.00000 \mathrm{E}+00$ & $0.00000 \mathrm{E}+00$ & 2.05294E-07 & $2.05002 \mathrm{E}-07$ & $2.02467 \mathrm{E}-07$ \\
\hline In-121 & $0.00000 \mathrm{E}+00$ & $0.00000 \mathrm{E}+00$ & $0.00000 \mathrm{E}+00$ & $3.10194 \mathrm{E}-06$ & $3.08962 \mathrm{E}-06$ & $3.06571 \mathrm{E}-06$ \\
\hline In-121m & $0.00000 \mathrm{E}+00$ & $0.00000 \mathrm{E}+00$ & $0.00000 \mathrm{E}+00$ & $1.10420 \mathrm{E}-05$ & $1.09966 \mathrm{E}-05$ & $1.09235 \mathrm{E}-05$ \\
\hline In-122 & $0.00000 \mathrm{E}+00$ & $0.00000 \mathrm{E}+00$ & $0.00000 \mathrm{E}+00$ & $2.32204 \mathrm{E}-07$ & $2.31895 \mathrm{E}-07$ & $2.30760 \mathrm{E}-07$ \\
\hline In- $122 m$ & $0.00000 \mathrm{E}+00$ & $0.00000 \mathrm{E}+00$ & $0.00000 \mathrm{E}+00$ & $2.99508 \mathrm{E}-07$ & $2.98547 \mathrm{E}-07$ & $2.94501 \mathrm{E}-07$ \\
\hline$I n-122 m$ & $0.00000 \mathrm{E}+00$ & $0.00000 \mathrm{E}+00$ & $0.00000 \mathrm{E}+00$ & $2.99508 \mathrm{E}-07$ & $2.98547 \mathrm{E}-07$ & $2.94501 \mathrm{E}-07$ \\
\hline In-123 & $0.00000 \mathrm{E}+00$ & $0.00000 \mathrm{E}+00$ & $0.00000 \mathrm{E}+00$ & $9.05232 \mathrm{E}-07$ & $9.07638 \mathrm{E}-07$ & $9.00752 \mathrm{E}-07$ \\
\hline$I n-123 m$ & $0.00000 \mathrm{E}+00$ & $0.00000 \mathrm{E}+00$ & $0.00000 \mathrm{E}+00$ & 2.79672E-06 & $2.79979 \mathrm{E}-06$ & $36 \mathrm{E}-06$ \\
\hline In-124 & $0.00000 \mathrm{E}+00$ & $0.00000 \mathrm{E}+00$ & $0.00000 \mathrm{E}+00$ & $4.24328 \mathrm{E}-07$ & $4.24668 \mathrm{E}-07$ & $4.23431 \mathrm{E}-07$ \\
\hline In- $124 m$ & $0.00000 \mathrm{E}+00$ & $0.00000 \mathrm{E}+00$ & $0.00000 \mathrm{E}+00$ & $2.80878 \mathrm{E}-07$ & $2.80347 \mathrm{E}-07$ & $2.76661 \mathrm{E}-07$ \\
\hline In-125 & $0.00000 \mathrm{E}+00$ & $0.00000 \mathrm{E}+00$ & $0.00000 \mathrm{E}+00$ & $2.91949 \mathrm{E}-07$ & $2.92190 \mathrm{E}-07$ & $2.90704 \mathrm{E}-07$ \\
\hline In- $125 m$ & $0.00000 \mathrm{E}+00$ & $0.00000 \mathrm{E}+00$ & $0.00000 \mathrm{E}+00$ & $1.21952 \mathrm{E}-06$ & $1.21930 \mathrm{E}-06$ & $1.20866 \mathrm{E}-06$ \\
\hline In-126 & $0.00000 \mathrm{E}+00$ & $0.00000 \mathrm{E}+00$ & $0.00000 \mathrm{E}+00$ & $1.77339 \mathrm{E}-07$ & $1.77289 \mathrm{E}-07$ & $1.76592 \mathrm{E}-07$ \\
\hline$I n-126 m$ & $0.00000 \mathrm{E}+00$ & $0.00000 \mathrm{E}+00$ & $0.00000 \mathrm{E}+00$ & $1.31244 \mathrm{E}-07$ & $1.30995 \mathrm{E}-07$ & $1.29599 \mathrm{E}-07$ \\
\hline $\operatorname{In}-127$ & $0.00000 \mathrm{E}+00$ & $0.00000 \mathrm{E}+00$ & $0.00000 \mathrm{E}+00$ & 3.61871E-07 & $3.62299 \mathrm{E}-07$ & $3.63335 \mathrm{E}-07$ \\
\hline$I n-127 m$ & $0.00000 \mathrm{E}+00$ & $0.00000 \mathrm{E}+00$ & $0.00000 \mathrm{E}+00$ & $5.17168 \mathrm{E}-07$ & $5.16350 \mathrm{E}-07$ & $5.11979 \mathrm{E}-07$ \\
\hline $\operatorname{In}-128$ & $0.00000 \mathrm{E}+00$ & $0.00000 \mathrm{E}+00$ & $0.00000 \mathrm{E}+00$ & $1.48797 \mathrm{E}-07$ & $1.47907 \mathrm{E}-07$ & $1.47107 \mathrm{E}-07$ \\
\hline In-128m & $0.00000 \mathrm{E}+00$ & $0.00000 \mathrm{E}+00$ & $0.00000 \mathrm{E}+00$ & $1.16093 \mathrm{E}-07$ & $1.15293 \mathrm{E}-07$ & $1.14479 \mathrm{E}-07$ \\
\hline In-129 & $0.00000 \mathrm{E}+00$ & $0.00000 \mathrm{E}+00$ & $0.00000 \mathrm{E}+00$ & $1.28086 \mathrm{E}-07$ & $1.29294 \mathrm{E}-07$ & 1.29533E-07 \\
\hline In-129m & $0.00000 \mathrm{E}+00$ & $0.00000 \mathrm{E}+00$ & $0.00000 \mathrm{E}+00$ & $2.44417 \mathrm{E}-07$ & $2.46769 \mathrm{E}-07$ & $2.47135 \mathrm{E}-07$ \\
\hline
\end{tabular}




\section{Depletion Analysis of Modular High Temperature Gas-cooled Reactor Loaded with LEU/Thorium Fuel}

February 2012

\begin{tabular}{|c|c|c|c|c|c|c|}
\hline \multirow[b]{2}{*}{ Nuclide } & \multicolumn{3}{|c|}{ Fresh Fuel } & \multicolumn{3}{|c|}{ Discharge } \\
\hline & Unit Cell & $\begin{array}{l}\text { Full Core } \\
(N O L B P)\end{array}$ & Full Core (3) & Unit Cell & $\begin{array}{l}\text { Full Core } \\
\text { (NO LBP) }\end{array}$ & Full Core (3) \\
\hline In-130 & $0.00000 \mathrm{E}+00$ & $0.00000 \mathrm{E}+00$ & $0.00000 \mathrm{E}+00$ & $1.30275 \mathrm{E}-07$ & $1.30626 \mathrm{E}-07$ & $1.31779 \mathrm{E}-07$ \\
\hline In-130m & $0.00000 \mathrm{E}+00$ & $0.00000 \mathrm{E}+00$ & $0.00000 \mathrm{E}+00$ & $2.71425 \mathrm{E}-08$ & $2.71035 \mathrm{E}-08$ & $2.68402 \mathrm{E}-08$ \\
\hline In-130m & $0.00000 \mathrm{E}+00$ & $0.00000 \mathrm{E}+00$ & $0.00000 \mathrm{E}+00$ & $2.71425 \mathrm{E}-08$ & $2.71035 \mathrm{E}-08$ & 2.68402E-08 \\
\hline In-131 & $0.00000 \mathrm{E}+00$ & $0.00000 \mathrm{E}+00$ & $0.00000 \mathrm{E}+00$ & $3.21953 \mathrm{E}-08$ & $3.21790 \mathrm{E}-08$ & $3.24196 \mathrm{E}-08$ \\
\hline In-131m & $0.00000 \mathrm{E}+00$ & $0.00000 \mathrm{E}+00$ & $0.00000 \mathrm{E}+00$ & $1.81578 \mathrm{E}-08$ & $1.80668 \mathrm{E}-08$ & $1.81202 \mathrm{E}-08$ \\
\hline In-132 & $0.00000 \mathrm{E}+00$ & $0.00000 \mathrm{E}+00$ & $0.00000 \mathrm{E}+00$ & 7.73583E-09 & 7.78563E-09 & 7.83264E-09 \\
\hline In-133 & $0.00000 \mathrm{E}+00$ & $0.00000 \mathrm{E}+00$ & $0.00000 \mathrm{E}+00$ & $2.27570 \mathrm{E}-10$ & $2.32665 \mathrm{E}-10$ & $2.33306 \mathrm{E}-10$ \\
\hline In-134 & $0.00000 \mathrm{E}+00$ & $0.00000 \mathrm{E}+00$ & $0.00000 \mathrm{E}+00$ & $5.24487 \mathrm{E}-12$ & $5.55905 \mathrm{E}-12$ & $53616 \mathrm{E}-12$ \\
\hline In-135 & $0.00000 \mathrm{E}+00$ & $0.00000 \mathrm{E}+00$ & $0.00000 \mathrm{E}+00$ & $4.21500 \mathrm{E}-14$ & $5.03070 \mathrm{E}-14$ & $90826 \mathrm{E}-14$ \\
\hline$S n-111$ & $0.00000 \mathrm{E}+00$ & $0.00000 \mathrm{E}+00$ & $0.00000 \mathrm{E}+00$ & $1.61970 \mathrm{E}-19$ & $1.62135 \mathrm{E}-19$ & $74130 \mathrm{E}-19$ \\
\hline$S n-113 m$ & $0.00000 \mathrm{E}+00$ & $0.00000 \mathrm{E}+00$ & $0.00000 \mathrm{E}+00$ & $1.50504 \mathrm{E}-18$ & $1.31951 \mathrm{E}-18$ & 63404E-18 \\
\hline$S n-117 m$ & $0.00000 \mathrm{E}+00$ & $0.00000 \mathrm{E}+00$ & $0.00000 \mathrm{E}+00$ & 9309E-04 & $2.17851 \mathrm{E}-04$ & $6217 \mathrm{E}-04$ \\
\hline$S n-119 m$ & $0.00000 \mathrm{E}+00$ & $0.00000 \mathrm{E}+00$ & $0.00000 \mathrm{E}+00$ & 0143E-04 & 2.69503E-04 & $+\mathrm{AL}-04$ \\
\hline$S n-121$ & $0.00000 \mathrm{E}+00$ & $0.00000 \mathrm{E}+00$ & $0.00000 \mathrm{E}+00$ & & $1.69108 \mathrm{E}-02$ & $5 \mathrm{E}-02$ \\
\hline$S n-121 m$ & $0.00000 \mathrm{E}+00$ & $0.00000 \mathrm{E}+00$ & $0.00000 \mathrm{E}+00$ & $2745 \mathrm{E}-01$ & $5.48812 \mathrm{E}-01$ & $3 \mathrm{E}-01$ \\
\hline$S n-123 m$ & $0.00000 \mathrm{E}+00$ & $0.00000 \mathrm{E}+00$ & $0.00000 \mathrm{E}+00$ & $7586 \mathrm{E}-04$ & $3.68475 \mathrm{E}-04$ & $6 \mathrm{E}-04$ \\
\hline$S n-125 m$ & $0.00000 \mathrm{E}+00$ & $0.00000 \mathrm{E}+00$ & $0.00000 \mathrm{E}+00$ & $1.22289 \mathrm{E}-04$ & $1.22303 \mathrm{E}-04$ & $4 \mathrm{E}-04$ \\
\hline$S n-127$ & $0.00000 \mathrm{E}+00$ & $0.00000 \mathrm{E}+00$ & $0.00000 \mathrm{E}+00$ & $1.58652 \mathrm{E}-02$ & $1.58605 \mathrm{E}-02$ & $1.57402 \mathrm{E}-02$ \\
\hline$S n-127 m$ & $0.00000 \mathrm{E}+00$ & $0.00000 \mathrm{E}+00$ & $0.00000 \mathrm{E}+00$ & $1.36824 \mathrm{E}-04$ & $1.36598 \mathrm{E}-04$ & $1.34627 \mathrm{E}-04$ \\
\hline$S n-128$ & $0.00000 \mathrm{E}+00$ & $0.00000 \mathrm{E}+00$ & $0.00000 \mathrm{E}+00$ & $1.45579 \mathrm{E}-02$ & $1.45569 \mathrm{E}-02$ & $1.44736 \mathrm{E}-02$ \\
\hline$S n-128 m$ & $0.00000 \mathrm{E}+00$ & $0.00000 \mathrm{E}+00$ & $0.00000 \mathrm{E}+00$ & $1.22493 \mathrm{E}-05$ & $1.22554 \mathrm{E}-05$ & $1.21857 \mathrm{E}-05$ \\
\hline Sn-129 & $0.00000 \mathrm{E}+00$ & $0.00000 \mathrm{E}+00$ & $0.00000 \mathrm{E}+00$ & $5.90379 \mathrm{E}-04$ & $5.90037 \mathrm{E}-04$ & $.85813 \mathrm{E}-04$ \\
\hline$S n-129 m$ & $0.00000 \mathrm{E}+00$ & $0.00000 \mathrm{E}+00$ & $0.00000 \mathrm{E}+00$ & $8.84579 \mathrm{E}-04$ & $8.83860 \mathrm{E}-04$ & 8.80449E-04 \\
\hline$S n-130$ & $0.00000 \mathrm{E}+00$ & $0.00000 \mathrm{E}+00$ & $0.00000 \mathrm{E}+00$ & $1.15348 \mathrm{E}-03$ & $1.15186 \mathrm{E}-03$ & 1.15297E-03 \\
\hline Sn-130m & $0.00000 \mathrm{E}+00$ & $0.00000 \mathrm{E}+00$ & $0.00000 \mathrm{E}+00$ & $4.71055 \mathrm{E}-04$ & 4.70207E-04 & 4.70406E-04 \\
\hline Sn-131 & $0.00000 \mathrm{E}+00$ & $0.00000 \mathrm{E}+00$ & $0.00000 \mathrm{E}+00$ & $2.09302 \mathrm{E}-04$ & $2.09035 \mathrm{E}-04$ & $2.09472 \mathrm{E}-04$ \\
\hline$S n-131 m$ & $0.00000 \mathrm{E}+00$ & $0.00000 \mathrm{E}+00$ & $0.00000 \mathrm{E}+00$ & $2.08528 \mathrm{E}-04$ & $2.08268 \mathrm{E}-04$ & 2.08664E-04 \\
\hline$S n-132$ & $0.00000 \mathrm{E}+00$ & $0.00000 \mathrm{E}+00$ & $0.00000 \mathrm{E}+00$ & $1.76710 \mathrm{E}-04$ & $1.76147 \mathrm{E}-04$ & $1.76470 \mathrm{E}-04$ \\
\hline$S n-133$ & $0.00000 \mathrm{E}+00$ & $0.00000 \mathrm{E}+00$ & $0.00000 \mathrm{E}+00$ & $1.33378 \mathrm{E}-06$ & $1.33540 \mathrm{E}-06$ & $1.34389 \mathrm{E}-06$ \\
\hline$S n-134$ & $0.00000 \mathrm{E}+00$ & $0.00000 \mathrm{E}+00$ & $0.00000 \mathrm{E}+00$ & $1.21707 \mathrm{E}-07$ & $1.22442 \mathrm{E}-07$ & $1.23180 \mathrm{E}-07$ \\
\hline$S n-135$ & $0.00000 \mathrm{E}+00$ & $0.00000 \mathrm{E}+00$ & $0.00000 \mathrm{E}+00$ & $2.52649 \mathrm{E}-09$ & $2.57346 \mathrm{E}-09$ & -09 \\
\hline$S n-136$ & $0.00000 \mathrm{E}+00$ & $0.00000 \mathrm{E}+00$ & $0.00000 \mathrm{E}+00$ & $3.69259 \mathrm{E}-11$ & 3.86604E-11 & 3.86217E-11 \\
\hline Sn-137 & $0.00000 \mathrm{E}+00$ & $0.00000 \mathrm{E}+00$ & $0.00000 \mathrm{E}+00$ & $1.73461 \mathrm{E}-11$ & $1.74549 \mathrm{E}-11$ & $1.76366 \mathrm{E}-11$ \\
\hline$S b-113$ & $0.00000 \mathrm{E}+00$ & $0.00000 \mathrm{E}+00$ & $0.00000 \mathrm{E}+00$ & $0.00000 \mathrm{E}+00$ & $0.00000 \mathrm{E}+00$ & $0.00000 \mathrm{E}+00$ \\
\hline$S b-115$ & $0.00000 \mathrm{E}+00$ & $0.00000 \mathrm{E}+00$ & $0.00000 \mathrm{E}+00$ & $0.00000 \mathrm{E}+00$ & $0.00000 \mathrm{E}+00$ & $0.00000 \mathrm{E}+00$ \\
\hline$S b-115 m$ & $0.00000 \mathrm{E}+00$ & $0.00000 \mathrm{E}+00$ & $0.00000 \mathrm{E}+00$ & $0.00000 \mathrm{E}+00$ & $0.00000 \mathrm{E}+00$ & $0.00000 \mathrm{E}+00$ \\
\hline
\end{tabular}




\begin{tabular}{|c|c|c|c|c|c|c|}
\hline \multirow[b]{2}{*}{ Nuclide } & \multicolumn{3}{|c|}{ Fresh Fuel } & \multicolumn{3}{|c|}{ Discharge } \\
\hline & Unit Cell & $\begin{array}{l}\text { Full Core } \\
\text { (NO LBP) }\end{array}$ & Full Core (3) & Unit Cell & $\begin{array}{l}\text { Full Core } \\
\text { (NO LBP) }\end{array}$ & Full Core (3) \\
\hline$S b-117$ & $0.00000 \mathrm{E}+00$ & $0.00000 \mathrm{E}+00$ & $0.00000 \mathrm{E}+00$ & $1.08833 \mathrm{E}-15$ & $1.13388 \mathrm{E}-15$ & $1.13995 \mathrm{E}-15$ \\
\hline$S b-118$ & $0.00000 \mathrm{E}+00$ & $0.00000 \mathrm{E}+00$ & $0.00000 \mathrm{E}+00$ & $3.02444 \mathrm{E}-15$ & $3.08181 \mathrm{E}-15$ & $3.04300 \mathrm{E}-15$ \\
\hline$S b-118 m$ & $0.00000 \mathrm{E}+00$ & $0.00000 \mathrm{E}+00$ & $0.00000 \mathrm{E}+00$ & $3.75441 \mathrm{E}-13$ & $3.82999 \mathrm{E}-13$ & $3.78365 \mathrm{E}-13$ \\
\hline$S b-119$ & $0.00000 \mathrm{E}+00$ & $0.00000 \mathrm{E}+00$ & $0.00000 \mathrm{E}+00$ & $5.40668 \mathrm{E}-10$ & $5.46637 \mathrm{E}-10$ & $5.37927 \mathrm{E}-10$ \\
\hline$S b-120$ & $0.00000 \mathrm{E}+00$ & $0.00000 \mathrm{E}+00$ & $0.00000 \mathrm{E}+00$ & $1.73290 \mathrm{E}-10$ & $1.95339 \mathrm{E}-10$ & $1.96775 \mathrm{E}-10$ \\
\hline$S b-120 m$ & $0.00000 \mathrm{E}+00$ & $0.00000 \mathrm{E}+00$ & $0.00000 \mathrm{E}+00$ & 4.94183E-08 & 4.97748E-08 & 4.89608E-08 \\
\hline$S b-122$ & $0.00000 \mathrm{E}+00$ & $0.00000 \mathrm{E}+00$ & $0.00000 \mathrm{E}+00$ & $4.41004 \mathrm{E}-03$ & $4.39281 \mathrm{E}-03$ & 4.30055E-03 \\
\hline$S b-122 m$ & $0.00000 \mathrm{E}+00$ & $0.00000 \mathrm{E}+00$ & $0.00000 \mathrm{E}+00$ & $1.99865 \mathrm{E}-08$ & $2.00463 \mathrm{E}-08$ & $96863 \mathrm{E}-08$ \\
\hline$S b-124 m$ & $0.00000 \mathrm{E}+00$ & $0.00000 \mathrm{E}+00$ & $0.00000 \mathrm{E}+00$ & $1.56555 \mathrm{E}-08$ & $1.54212 \mathrm{E}-08$ & $33 \mathrm{E}-08$ \\
\hline$S b-124 m$ & $0.00000 \mathrm{E}+00$ & $0.00000 \mathrm{E}+00$ & $0.00000 \mathrm{E}+00$ & $1.56555 \mathrm{E}-08$ & $1.54212 \mathrm{E}-08$ & $33 \mathrm{E}-08$ \\
\hline$S b-126 m$ & $0.00000 \mathrm{E}+00$ & $0.00000 \mathrm{E}+00$ & $0.00000 \mathrm{E}+00$ & $9 \mathrm{E}-06$ & $8.11979 \mathrm{E}-06$ & 8.09224E-06 \\
\hline$S b-126 m$ & $0.00000 \mathrm{E}+00$ & $0.00000 \mathrm{E}+00$ & $0.00000 \mathrm{E}+00$ & $8.13829 \mathrm{E}-06$ & 8.11979E-06 & 8.09224E-06 \\
\hline$S b-127$ & $0.00000 \mathrm{E}+00$ & $0.00000 \mathrm{E}+00$ & $0.00000 \mathrm{E}+00$ & $9.23573 \mathrm{E}-01$ & $9.22895 \mathrm{E}-01$ & $9.14787 \mathrm{E}-01$ \\
\hline$S b-128$ & $0.00000 \mathrm{E}+00$ & $0.00000 \mathrm{E}+00$ & $0.00000 \mathrm{E}+00$ & $1.43249 \mathrm{E}-01$ & $1.43251 \mathrm{E}-01$ & $1.42360 \mathrm{E}-01$ \\
\hline$S b-128 m$ & $0.00000 \mathrm{E}+00$ & $0.00000 \mathrm{E}+00$ & $0.00000 \mathrm{E}+00$ & $1.62537 \mathrm{E}-04$ & $1.62560 \mathrm{E}-04$ & $1.60267 \mathrm{E}-04$ \\
\hline$S b-129$ & $0.00000 \mathrm{E}+00$ & $0.00000 \mathrm{E}+00$ & $0.00000 \mathrm{E}+00$ & $1.33077 \mathrm{E}-01$ & $1.33003 \mathrm{E}-01$ & $1.32010 \mathrm{E}-01$ \\
\hline$S b-130$ & $0.00000 \mathrm{E}+00$ & $0.00000 \mathrm{E}+00$ & $0.00000 \mathrm{E}+00$ & $1.90335 \mathrm{E}-02$ & $1.89862 \mathrm{E}-02$ & $1.89339 \mathrm{E}-02$ \\
\hline$S b-130 m$ & $0.00000 \mathrm{E}+00$ & $0.00000 \mathrm{E}+00$ & $0.00000 \mathrm{E}+00$ & $3.36989 \mathrm{E}-03$ & 3.36567E-03 & 3.35984E-03 \\
\hline$S b-131$ & $0.00000 \mathrm{E}+00$ & $0.00000 \mathrm{E}+00$ & $0.00000 \mathrm{E}+00$ & $3.13209 \mathrm{E}-02$ & $3.12502 \mathrm{E}-02$ & $.12394 \mathrm{E}-02$ \\
\hline$S b-132$ & $0.00000 \mathrm{E}+00$ & $0.00000 \mathrm{E}+00$ & $0.00000 \mathrm{E}+00$ & $2.81529 \mathrm{E}-03$ & $2.80108 \mathrm{E}-03$ & $.79813 \mathrm{E}-03$ \\
\hline$S b-132 m$ & $0.00000 \mathrm{E}+00$ & $0.00000 \mathrm{E}+00$ & $0.00000 \mathrm{E}+00$ & $1.68560 \mathrm{E}-03$ & $1.68259 \mathrm{E}-03$ & $1.68691 \mathrm{E}-03$ \\
\hline$S b-133$ & $0.00000 \mathrm{E}+00$ & $0.00000 \mathrm{E}+00$ & $0.00000 \mathrm{E}+00$ & $2.62667 \mathrm{E}-03$ & $2.62076 \mathrm{E}-03$ & $25 \mathrm{E}-03$ \\
\hline$S b-134$ & $0.00000 \mathrm{E}+00$ & $0.00000 \mathrm{E}+00$ & $0.00000 \mathrm{E}+00$ & $2.25530 \mathrm{E}-06$ & $2.24949 \mathrm{E}-06$ & $.25840 \mathrm{E}-06$ \\
\hline$S b-134 m$ & $0.00000 \mathrm{E}+00$ & $0.00000 \mathrm{E}+00$ & $0.00000 \mathrm{E}+00$ & 2.79493E-05 & $2.78672 \mathrm{E}-05$ & $79752 \mathrm{E}-05$ \\
\hline$S b-135$ & $0.00000 \mathrm{E}+00$ & $0.00000 \mathrm{E}+00$ & $0.00000 \mathrm{E}+00$ & $1.88279 \mathrm{E}-06$ & $1.88411 \mathrm{E}-06$ & $1.89210 \mathrm{E}-06$ \\
\hline$S b-136$ & $0.00000 \mathrm{E}+00$ & $0.00000 \mathrm{E}+00$ & $0.00000 \mathrm{E}+00$ & $9.12564 \mathrm{E}-08$ & $9.18531 \mathrm{E}-08$ & $.22983 \mathrm{E}-08$ \\
\hline$S b-137$ & $0.00000 \mathrm{E}+00$ & $0.00000 \mathrm{E}+00$ & $0.00000 \mathrm{E}+00$ & $7.16149 \mathrm{E}-08$ & $7.18636 \mathrm{E}-08$ & $.26535 \mathrm{E}-08$ \\
\hline$S b-138$ & $0.00000 \mathrm{E}+00$ & $0.00000 \mathrm{E}+00$ & $0.00000 \mathrm{E}+00$ & $7.85750 \mathrm{E}-11$ & $8.16815 \mathrm{E}-11$ & $8.16519 \mathrm{E}-11$ \\
\hline$S b-139$ & $0.00000 \mathrm{E}+00$ & $0.00000 \mathrm{E}+00$ & $0.00000 \mathrm{E}+00$ & $2.92692 \mathrm{E}-12$ & $3.10767 \mathrm{E}-12$ & $3.09511 \mathrm{E}-12$ \\
\hline Te-115 & $0.00000 \mathrm{E}+00$ & $0.00000 \mathrm{E}+00$ & $0.00000 \mathrm{E}+00$ & $0.00000 \mathrm{E}+00$ & $0.00000 \mathrm{E}+00$ & $0.00000 \mathrm{E}+00$ \\
\hline Te-117 & $0.00000 \mathrm{E}+00$ & $0.00000 \mathrm{E}+00$ & $0.00000 \mathrm{E}+00$ & $0.00000 \mathrm{E}+00$ & $0.00000 \mathrm{E}+00$ & $0.00000 \mathrm{E}+00$ \\
\hline Te-118 & $0.00000 \mathrm{E}+00$ & $0.00000 \mathrm{E}+00$ & $0.00000 \mathrm{E}+00$ & $0.00000 \mathrm{E}+00$ & $0.00000 \mathrm{E}+00$ & $0.00000 \mathrm{E}+00$ \\
\hline Te-119 & $0.00000 \mathrm{E}+00$ & $0.00000 \mathrm{E}+00$ & $0.00000 \mathrm{E}+00$ & $1.05853 \mathrm{E}-15$ & $1.08461 \mathrm{E}-15$ & $1.09476 \mathrm{E}-15$ \\
\hline$T e-121$ & $0.00000 \mathrm{E}+00$ & $0.00000 \mathrm{E}+00$ & $0.00000 \mathrm{E}+00$ & $8.56588 \mathrm{E}-09$ & 9.67449E-09 & 9.92899E-09 \\
\hline $\mathrm{Te}-121 \mathrm{~m}$ & $0.00000 \mathrm{E}+00$ & $0.00000 \mathrm{E}+00$ & $0.00000 \mathrm{E}+00$ & $8.61662 \mathrm{E}-09$ & 8.61912E-09 & $8.68410 \mathrm{E}-09$ \\
\hline$T e-123 m$ & $0.00000 \mathrm{E}+00$ & $0.00000 \mathrm{E}+00$ & $0.00000 \mathrm{E}+00$ & $1.93859 \mathrm{E}-05$ & $1.89493 \mathrm{E}-05$ & $1.89362 \mathrm{E}-05$ \\
\hline
\end{tabular}




\section{Depletion Analysis of Modular High Temperature Gas-cooled Reactor Loaded with LEU/Thorium Fuel}

February 2012

61

\begin{tabular}{|c|c|c|c|c|c|c|}
\hline \multirow[b]{2}{*}{ Nuclide } & \multicolumn{3}{|c|}{ Fresh Fuel } & \multicolumn{3}{|c|}{ Discharge } \\
\hline & Unit Cell & $\begin{array}{l}\text { Full Core } \\
\text { (NO LBP) }\end{array}$ & Full Core (3) & Unit Cell & $\begin{array}{l}\text { Full Core } \\
\text { (NO LBP) }\end{array}$ & Full Core (3) \\
\hline$T e-125 m$ & $0.00000 \mathrm{E}+00$ & $0.00000 \mathrm{E}+00$ & $0.00000 \mathrm{E}+00$ & $2.50108 \mathrm{E}-01$ & $2.48473 \mathrm{E}-01$ & $2.52832 \mathrm{E}-01$ \\
\hline Te-127 & $0.00000 \mathrm{E}+00$ & $0.00000 \mathrm{E}+00$ & $0.00000 \mathrm{E}+00$ & $9.05622 \mathrm{E}-02$ & $9.04680 \mathrm{E}-02$ & $8.97224 \mathrm{E}-02$ \\
\hline Te-129 & $0.00000 \mathrm{E}+00$ & $0.00000 \mathrm{E}+00$ & $0.00000 \mathrm{E}+00$ & $3.52409 \mathrm{E}-02$ & $3.52265 \mathrm{E}-02$ & $3.49630 \mathrm{E}-02$ \\
\hline Te-131 & $0.00000 \mathrm{E}+00$ & $0.00000 \mathrm{E}+00$ & $0.00000 \mathrm{E}+00$ & $3.89631 \mathrm{E}-02$ & $3.88878 \mathrm{E}-02$ & $3.88189 \mathrm{E}-02$ \\
\hline$T e-131 m$ & $0.00000 \mathrm{E}+00$ & $0.00000 \mathrm{E}+00$ & $0.00000 \mathrm{E}+00$ & $5.11666 \mathrm{E}-01$ & $5.12431 \mathrm{E}-01$ & $5.06890 \mathrm{E}-01$ \\
\hline Te-133 & $0.00000 \mathrm{E}+00$ & $0.00000 \mathrm{E}+00$ & $0.00000 \mathrm{E}+00$ & $2.59146 \mathrm{E}-02$ & $2.58581 \mathrm{E}-02$ & $2.58737 \mathrm{E}-02$ \\
\hline$T e-133 m$ & $0.00000 \mathrm{E}+00$ & $0.00000 \mathrm{E}+00$ & $0.00000 \mathrm{E}+00$ & $9.13441 \mathrm{E}-02$ & $9.12550 \mathrm{E}-02$ & $9.11712 \mathrm{E}-02$ \\
\hline Te-134 & $0.00000 \mathrm{E}+00$ & $0.00000 \mathrm{E}+00$ & $0.00000 \mathrm{E}+00$ & $1.38871 \mathrm{E}-01$ & $1.38667 \mathrm{E}-01$ & $1.38910 \mathrm{E}-01$ \\
\hline Te-135 & $0.00000 \mathrm{E}+00$ & $0.00000 \mathrm{E}+00$ & $0.00000 \mathrm{E}+00$ & $5.18215 \mathrm{E}-04$ & 5.17073E-04 & 8232E-04 \\
\hline Te-136 & $0.00000 \mathrm{E}+00$ & $0.00000 \mathrm{E}+00$ & $0.00000 \mathrm{E}+00$ & $1.80743 \mathrm{E}-04$ & $1.80748 \mathrm{E}-04$ & $1.81517 \mathrm{E}-04$ \\
\hline Te-137 & $0.00000 \mathrm{E}+00$ & $0.00000 \mathrm{E}+00$ & $0.00000 \mathrm{E}+00$ & $7.90811 \mathrm{E}-06$ & $7.90248 \mathrm{E}-06$ & 95314E-06 \\
\hline Te-138 & $0.00000 \mathrm{E}+00$ & $0.00000 \mathrm{E}+00$ & $0.00000 \mathrm{E}+00$ & $6.87472 \mathrm{E}-07$ & $6.90086 \mathrm{E}-07$ & 4430E-07 \\
\hline Te-139 & $0.00000 \mathrm{E}+00$ & $0.00000 \mathrm{E}+00$ & $0.00000 \mathrm{E}+00$ & & $1.94775 \mathrm{E}-08$ & $8 \mathrm{E}-08$ \\
\hline Te-140 & $0.00000 \mathrm{E}+00$ & $0.00000 \mathrm{E}+00$ & $0.00000 \mathrm{E}+00$ & $7 \mathrm{E}-08$ & $2.58201 \mathrm{E}-08$ & $2.60976 \mathrm{E}-08$ \\
\hline Te-141 & $0.00000 \mathrm{E}+00$ & $0.00000 \mathrm{E}+00$ & $0.00000 \mathrm{E}+00$ & 7.38834E-11 & $8.03388 \mathrm{E}-11$ & $7.96445 \mathrm{E}-11$ \\
\hline Te-142 & $0.00000 \mathrm{E}+00$ & $0.00000 \mathrm{E}+00$ & $0.00000 \mathrm{E}+00$ & $3.55333 \mathrm{E}-12$ & $3.63036 \mathrm{E}-12$ & $3.64029 \mathrm{E}-12$ \\
\hline$I-121$ & $0.00000 \mathrm{E}+00$ & $0.00000 \mathrm{E}+00$ & $0.00000 \mathrm{E}+00$ & $5.43378 \mathrm{E}-17$ & $5.39983 \mathrm{E}-17$ & $5.67877 \mathrm{E}-17$ \\
\hline$I-122$ & $0.00000 \mathrm{E}+00$ & $0.00000 \mathrm{E}+00$ & $0.00000 \mathrm{E}+00$ & $0.00000 \mathrm{E}+00$ & $0.00000 \mathrm{E}+00$ & $0.00000 \mathrm{E}+00$ \\
\hline$I-123$ & $0.00000 \mathrm{E}+00$ & $0.00000 \mathrm{E}+00$ & $0.00000 \mathrm{E}+00$ & $1.13122 \mathrm{E}-11$ & $1.11225 \mathrm{E}-11$ & $1.08576 \mathrm{E}-11$ \\
\hline$I-124$ & $0.00000 \mathrm{E}+00$ & $0.00000 \mathrm{E}+00$ & $0.00000 \mathrm{E}+00$ & $2.90509 \mathrm{E}-20$ & 4.39293E-20 & $4.71355 \mathrm{E}-20$ \\
\hline$I-125$ & $0.00000 \mathrm{E}+00$ & $0.00000 \mathrm{E}+00$ & $0.00000 \mathrm{E}+00$ & $1.58488 \mathrm{E}-12$ & $2.05475 \mathrm{E}-12$ & $2.11338 \mathrm{E}-12$ \\
\hline$I-126$ & $0.00000 \mathrm{E}+00$ & $0.00000 \mathrm{E}+00$ & $0.00000 \mathrm{E}+00$ & $2.45399 \mathrm{E}-06$ & $2.97698 \mathrm{E}-06$ & $3.03101 \mathrm{E}-06$ \\
\hline$I-128$ & $0.00000 \mathrm{E}+00$ & $0.00000 \mathrm{E}+00$ & $0.00000 \mathrm{E}+00$ & $3.56826 \mathrm{E}-04$ & $3.67620 \mathrm{E}-04$ & 3.61421E-04 \\
\hline $\mathrm{I}-130 \mathrm{~m}$ & $0.00000 \mathrm{E}+00$ & $0.00000 \mathrm{E}+00$ & $0.00000 \mathrm{E}+00$ & $2.35551 \mathrm{E}-06$ & $2.33883 \mathrm{E}-06$ & $2.29556 \mathrm{E}-06$ \\
\hline I-132 & $0.00000 \mathrm{E}+00$ & $0.00000 \mathrm{E}+00$ & $0.00000 \mathrm{E}+00$ & $3.59628 \mathrm{E}-01$ & $3.59125 \mathrm{E}-01$ & $3.58260 \mathrm{E}-01$ \\
\hline$I-132 m$ & $0.00000 \mathrm{E}+00$ & $0.00000 \mathrm{E}+00$ & $0.00000 \mathrm{E}+00$ & $2.51015 \mathrm{E}-03$ & $2.49698 \mathrm{E}-03$ & $2.45793 \mathrm{E}-03$ \\
\hline$I-133$ & $0.00000 \mathrm{E}+00$ & $0.00000 \mathrm{E}+00$ & $0.00000 \mathrm{E}+00$ & $4.61337 \mathrm{E}+00$ & $4.60501 \mathrm{E}+00$ & $4.60049 \mathrm{E}+00$ \\
\hline$I-133 m$ & $0.00000 \mathrm{E}+00$ & $0.00000 \mathrm{E}+00$ & $0.00000 \mathrm{E}+00$ & $1.93793 \mathrm{E}-05$ & $1.93093 \mathrm{E}-05$ & $1.90310 \mathrm{E}-05$ \\
\hline$I-134$ & $0.00000 \mathrm{E}+00$ & $0.00000 \mathrm{E}+00$ & $0.00000 \mathrm{E}+00$ & $2.18845 \mathrm{E}-01$ & $2.18420 \mathrm{E}-01$ & $2.18360 \mathrm{E}-01$ \\
\hline$I-134 m$ & $0.00000 \mathrm{E}+00$ & $0.00000 \mathrm{E}+00$ & $0.00000 \mathrm{E}+00$ & $1.30472 \mathrm{E}-03$ & $1.29914 \mathrm{E}-03$ & $1.28780 \mathrm{E}-03$ \\
\hline$I-136$ & $0.00000 \mathrm{E}+00$ & $0.00000 \mathrm{E}+00$ & $0.00000 \mathrm{E}+00$ & $1.91358 \mathrm{E}-03$ & $1.91046 \mathrm{E}-03$ & $1.91424 \mathrm{E}-03$ \\
\hline$I-136 m$ & $0.00000 \mathrm{E}+00$ & $0.00000 \mathrm{E}+00$ & $0.00000 \mathrm{E}+00$ & $6.19747 \mathrm{E}-04$ & $6.16948 \mathrm{E}-04$ & $6.15918 \mathrm{E}-04$ \\
\hline$I-137$ & $0.00000 \mathrm{E}+00$ & $0.00000 \mathrm{E}+00$ & $0.00000 \mathrm{E}+00$ & $6.53447 \mathrm{E}-04$ & $6.51331 \mathrm{E}-04$ & $6.52351 \mathrm{E}-04$ \\
\hline$I-138$ & $0.00000 \mathrm{E}+00$ & $0.00000 \mathrm{E}+00$ & $0.00000 \mathrm{E}+00$ & $8.24676 \mathrm{E}-05$ & $8.21216 \mathrm{E}-05$ & $8.22430 \mathrm{E}-05$ \\
\hline$I-139$ & $0.00000 \mathrm{E}+00$ & $0.00000 \mathrm{E}+00$ & $0.00000 \mathrm{E}+00$ & $1.31328 \mathrm{E}-05$ & $1.31142 \mathrm{E}-05$ & $1.31787 \mathrm{E}-05$ \\
\hline$I-140$ & $0.00000 \mathrm{E}+00$ & $0.00000 \mathrm{E}+00$ & $0.00000 \mathrm{E}+00$ & $9.85356 \mathrm{E}-07$ & $9.84913 \mathrm{E}-07$ & $9.90178 \mathrm{E}-07$ \\
\hline
\end{tabular}




\begin{tabular}{|c|c|c|c|c|c|c|}
\hline \multirow[b]{2}{*}{ Nuclide } & \multicolumn{3}{|c|}{ Fresh Fuel } & \multicolumn{3}{|c|}{ Discharge } \\
\hline & Unit Cell & $\begin{array}{l}\text { Full Core } \\
\text { (NO LBP) }\end{array}$ & Full Core (3) & Unit Cell & $\begin{array}{l}\text { Full Core } \\
(N O L B P)\end{array}$ & Full Core (3) \\
\hline$I-141$ & $0.00000 \mathrm{E}+00$ & $0.00000 \mathrm{E}+00$ & $0.00000 \mathrm{E}+00$ & $1.11165 \mathrm{E}-07$ & $1.11541 \mathrm{E}-07$ & $1.12381 \mathrm{E}-07$ \\
\hline$I-142$ & $0.00000 \mathrm{E}+00$ & $0.00000 \mathrm{E}+00$ & $0.00000 \mathrm{E}+00$ & $8.85320 \mathrm{E}-09$ & $8.84035 \mathrm{E}-09$ & 8.88933E-09 \\
\hline$I-143$ & $0.00000 \mathrm{E}+00$ & $0.00000 \mathrm{E}+00$ & $0.00000 \mathrm{E}+00$ & $1.09685 \mathrm{E}-10$ & $1.22054 \mathrm{E}-10$ & $1.20382 \mathrm{E}-10$ \\
\hline$I-144$ & $0.00000 \mathrm{E}+00$ & $0.00000 \mathrm{E}+00$ & $0.00000 \mathrm{E}+00$ & $3.88750 \mathrm{E}-12$ & $3.94852 \mathrm{E}-12$ & $3.95771 \mathrm{E}-12$ \\
\hline $\mathrm{Xe}-122$ & $0.00000 \mathrm{E}+00$ & $0.00000 \mathrm{E}+00$ & $0.00000 \mathrm{E}+00$ & $0.00000 \mathrm{E}+00$ & $0.00000 \mathrm{E}+00$ & $0.00000 \mathrm{E}+00$ \\
\hline$X e-125$ & $0.00000 \mathrm{E}+00$ & $0.00000 \mathrm{E}+00$ & $0.00000 \mathrm{E}+00$ & $9.98404 \mathrm{E}-15$ & $1.39625 \mathrm{E}-14$ & $1.51490 \mathrm{E}-14$ \\
\hline$X e-125 m$ & $0.00000 \mathrm{E}+00$ & $0.00000 \mathrm{E}+00$ & $0.00000 \mathrm{E}+00$ & $0.00000 \mathrm{E}+00$ & $0.00000 \mathrm{E}+00$ & $0.00000 \mathrm{E}+00$ \\
\hline$X e-127$ & $0.00000 \mathrm{E}+00$ & $0.00000 \mathrm{E}+00$ & $0.00000 \mathrm{E}+00$ & $2.09884 \mathrm{E}-07$ & $2.54150 \mathrm{E}-07$ & $2.63186 \mathrm{E}-07$ \\
\hline$X e-127 m$ & $0.00000 \mathrm{E}+00$ & $0.00000 \mathrm{E}+00$ & $0.00000 \mathrm{E}+00$ & $0.00000 \mathrm{E}+00$ & $0.00000 \mathrm{E}+00$ & $0.00000 \mathrm{E}+00$ \\
\hline $\mathrm{Xe}-129 \mathrm{~m}$ & $0.00000 \mathrm{E}+00$ & $0.00000 \mathrm{E}+00$ & $0.00000 \mathrm{E}+00$ & $5.55588 \mathrm{E}-09$ & $5.72379 \mathrm{E}-09$ & 5.66514E-09 \\
\hline $\mathrm{Xe}-131 \mathrm{~m}$ & $0.00000 \mathrm{E}+00$ & $0.00000 \mathrm{E}+00$ & $0000 \mathrm{E}+00$ & $7347 \mathrm{E}-01$ & $3.66907 \mathrm{E}-01$ & $3 \mathrm{E}-01$ \\
\hline$X e-133 m$ & $0.00000 \mathrm{E}+00$ & $0.00000 \mathrm{E}+00$ & $0.00000 \mathrm{E}+00$ & $68 \mathrm{E}-01$ & $1.49094 \mathrm{E}-01$ & $9 \mathrm{E}-01$ \\
\hline$X e-134 m$ & $0.00000 \mathrm{E}+00$ & $0.00000 \mathrm{E}+00$ & $0.00000 \mathrm{E}+00$ & $2.45653 \mathrm{E}-07$ & $2.44697 \mathrm{E}-07$ & $2.41501 \mathrm{E}-07$ \\
\hline$X e-135 m$ & $0.00000 \mathrm{E}+00$ & $0.00000 \mathrm{E}+00$ & $0.00000 \mathrm{E}+00$ & $8.86727 \mathrm{E}-03$ & $8.85272 \mathrm{E}-03$ & $8.80863 \mathrm{E}-03$ \\
\hline$X e-137$ & $0.00000 \mathrm{E}+00$ & $0.00000 \mathrm{E}+00$ & $0.00000 \mathrm{E}+00$ & $1.37276 \mathrm{E}-02$ & $1.37099 \mathrm{E}-02$ & $1.36990 \mathrm{E}-02$ \\
\hline $\mathrm{Xe}-138$ & $0.00000 \mathrm{E}+00$ & $0.00000 \mathrm{E}+00$ & $0.00000 \mathrm{E}+00$ & 4.73293E-02 & $4.72665 \mathrm{E}-02$ & $4.72871 \mathrm{E}-02$ \\
\hline$X e-139$ & $0.00000 \mathrm{E}+00$ & $0.00000 \mathrm{E}+00$ & $0.00000 \mathrm{E}+00$ & $1.65509 \mathrm{E}-03$ & $1.65355 \mathrm{E}-03$ & $1.65729 \mathrm{E}-03$ \\
\hline$X e-140$ & $0.00000 \mathrm{E}+00$ & $0.00000 \mathrm{E}+00$ & $0.00000 \mathrm{E}+00$ & $3.72874 \mathrm{E}-04$ & $3.72704 \mathrm{E}-04$ & $3.74218 \mathrm{E}-04$ \\
\hline$X e-141$ & $0.00000 \mathrm{E}+00$ & $0.00000 \mathrm{E}+00$ & $0.00000 \mathrm{E}+00$ & $1.62437 \mathrm{E}-05$ & $1.62226 \mathrm{E}-05$ & $2 \mathrm{E}-05$ \\
\hline$X e-142$ & $0.00000 \mathrm{E}+00$ & $0.00000 \mathrm{E}+00$ & $0.00000 \mathrm{E}+00$ & $4.05610 \mathrm{E}-06$ & $4.05410 \mathrm{E}-06$ & $07788 \mathrm{E}-06$ \\
\hline$X e-143$ & $0.00000 \mathrm{E}+00$ & $0.00000 \mathrm{E}+00$ & $0.00000 \mathrm{E}+00$ & $1.05834 \mathrm{E}-07$ & $1.05887 \mathrm{E}-07$ & $1.06626 \mathrm{E}-07$ \\
\hline$X e-144$ & $0.00000 \mathrm{E}+00$ & $0.00000 \mathrm{E}+00$ & $0.00000 \mathrm{E}+00$ & $1.92943 \mathrm{E}-08$ & $1.94831 \mathrm{E}-08$ & 02E-08 \\
\hline $\mathrm{Xe}-145$ & $0.00000 \mathrm{E}+00$ & $0.00000 \mathrm{E}+00$ & $0.00000 \mathrm{E}+00$ & $3.38348 \mathrm{E}-10$ & $3.49791 \mathrm{E}-10$ & $9 \mathrm{E}-10$ \\
\hline $\mathrm{Xe}-146$ & $0.00000 \mathrm{E}+00$ & $0.00000 \mathrm{E}+00$ & $0.00000 \mathrm{E}+00$ & $1.84437 \mathrm{E}-11$ & $1.91719 \mathrm{E}-11$ & 727E-11 \\
\hline$X e-147$ & $0.00000 \mathrm{E}+00$ & $0.00000 \mathrm{E}+00$ & $0.00000 \mathrm{E}+00$ & $1.62419 \mathrm{E}-12$ & $1.62573 \mathrm{E}-12$ & $1.64161 \mathrm{E}-12$ \\
\hline Cs-127 & $0.00000 \mathrm{E}+00$ & $0.00000 \mathrm{E}+00$ & $0.00000 \mathrm{E}+00$ & $0.00000 \mathrm{E}+00$ & $0.00000 \mathrm{E}+00$ & $0.00000 \mathrm{E}+00$ \\
\hline Cs -128 & $0.00000 \mathrm{E}+00$ & $0.00000 \mathrm{E}+00$ & $0.00000 \mathrm{E}+00$ & $0.00000 \mathrm{E}+00$ & $0.00000 \mathrm{E}+00$ & $0.00000 \mathrm{E}+00$ \\
\hline Cs-129 & $0.00000 \mathrm{E}+00$ & $0.00000 \mathrm{E}+00$ & $0.00000 \mathrm{E}+00$ & $1.29766 \mathrm{E}-15$ & $1.38691 \mathrm{E}-15$ & $1.47213 \mathrm{E}-15$ \\
\hline Cs-130 & $0.00000 \mathrm{E}+00$ & $0.00000 \mathrm{E}+00$ & $0.00000 \mathrm{E}+00$ & $2.55771 \mathrm{E}-22$ & $3.79310 \mathrm{E}-22$ & $3.96488 \mathrm{E}-22$ \\
\hline Cs-131 & $0.00000 \mathrm{E}+00$ & $0.00000 \mathrm{E}+00$ & $0.00000 \mathrm{E}+00$ & & $6.67701 \mathrm{E}-09$ & $6.55505 \mathrm{E}-09$ \\
\hline Cs-132 & $0.00000 \mathrm{E}+00$ & $0.00000 \mathrm{E}+00$ & $0.00000 \mathrm{E}+00$ & 2.79779E-05 & $3.37916 \mathrm{E}-05$ & $3.39901 \mathrm{E}-05$ \\
\hline Cs $-134 m$ & $0.00000 \mathrm{E}+00$ & $0.00000 \mathrm{E}+00$ & $0.00000 \mathrm{E}+00$ & $1.01672 \mathrm{E}-05$ & $1.00642 \mathrm{E}-05$ & $9.85960 \mathrm{E}-06$ \\
\hline Cs $-135 m$ & $0.00000 \mathrm{E}+00$ & $0.00000 \mathrm{E}+00$ & $0.00000 \mathrm{E}+00$ & $6.35403 \mathrm{E}-05$ & $6.30005 \mathrm{E}-05$ & $6.18286 \mathrm{E}-05$ \\
\hline Cs- $136 m$ & $0.00000 \mathrm{E}+00$ & $0.00000 \mathrm{E}+00$ & $0.00000 \mathrm{E}+00$ & 4.36097E-06 & $4.36800 \mathrm{E}-06$ & $4.29940 \mathrm{E}-06$ \\
\hline Cs-138 & $0.00000 \mathrm{E}+00$ & $0.00000 \mathrm{E}+00$ & $0.00000 \mathrm{E}+00$ & $1.23359 \mathrm{E}-01$ & $1.23216 \mathrm{E}-01$ & $1.23195 \mathrm{E}-01$ \\
\hline Cs $-138 m$ & $0.00000 \mathrm{E}+00$ & $0.00000 \mathrm{E}+00$ & $0.00000 \mathrm{E}+00$ & 6.51976E-04 & $6.51846 \mathrm{E}-04$ & $6.46414 \mathrm{E}-04$ \\
\hline
\end{tabular}




\section{Depletion Analysis of Modular High Temperature Gas-cooled Reactor Loaded with LEU/Thorium Fuel}

February 2012

63

\begin{tabular}{|c|c|c|c|c|c|c|}
\hline \multirow[b]{2}{*}{ Nuclide } & \multicolumn{3}{|c|}{ Fresh Fuel } & \multicolumn{3}{|c|}{ Discharge } \\
\hline & Unit Cell & $\begin{array}{l}\text { Full Core } \\
(N O L B P)\end{array}$ & Full Core (3) & Unit Cell & $\begin{array}{l}\text { Full Core } \\
\text { (NO LBP) }\end{array}$ & Full Core (3) \\
\hline 139 & $0.00000 \mathrm{E}+00$ & $0.00000 \mathrm{E}+00$ & $0.00000 \mathrm{E}+00$ & $3.26870 \mathrm{E}-02$ & $3.26631 \mathrm{E}-02$ & $3.26634 \mathrm{E}-02$ \\
\hline$s-140$ & $0.00000 \mathrm{E}+00$ & $0.00000 \mathrm{E}+00$ & $0.00000 \mathrm{E}+00$ & $3.16224 \mathrm{E}-03$ & 3.16127E-03 & 3.16549E-03 \\
\hline Cs-141 & $0.00000 \mathrm{E}+00$ & $0.00000 \mathrm{E}+00$ & $0.00000 \mathrm{E}+00$ & $9.54988 \mathrm{E}-04$ & $9.54500 \mathrm{E}-04$ & $9.54836 \mathrm{E}-04$ \\
\hline$C s-142$ & $0.00000 \mathrm{E}+00$ & $0.00000 \mathrm{E}+00$ & $0.00000 \mathrm{E}+00$ & $3.79075 \mathrm{E}-05$ & $3.78395 \mathrm{E}-05$ & $3.79562 \mathrm{E}-05$ \\
\hline$C s-143$ & $0.00000 \mathrm{E}+00$ & $0.00000 \mathrm{E}+00$ & $0.00000 \mathrm{E}+00$ & $1.98879 \mathrm{E}-05$ & $1.98445 \mathrm{E}-05$ & $1.99318 \mathrm{E}-05$ \\
\hline Cs-144 & $0.00000 \mathrm{E}+00$ & $0.00000 \mathrm{E}+00$ & $0.00000 \mathrm{E}+00$ & $3.35875 \mathrm{E}-06$ & $3.35419 \mathrm{E}-06$ & 3.37231E-06 \\
\hline$C s-145$ & $0.00000 \mathrm{E}+00$ & $0.00000 \mathrm{E}+00$ & $0.00000 \mathrm{E}+00$ & $3.84995 \mathrm{E}-07$ & $3.85367 \mathrm{E}-07$ & 3.87244E-07 \\
\hline$C s-146$ & $0.00000 \mathrm{E}+00$ & $0.00000 \mathrm{E}+00$ & $0.00000 \mathrm{E}+00$ & $2.34588 \mathrm{E}-08$ & $2.35177 \mathrm{E}-08$ & 36491E-08 \\
\hline$C s-147$ & $0.00000 \mathrm{E}+00$ & $0.00000 \mathrm{E}+00$ & $0.00000 \mathrm{E}+00$ & $3.71291 \mathrm{E}-09$ & $3.71361 \mathrm{E}-09$ & $75009 \mathrm{E}-09$ \\
\hline$C s-148$ & $0.00000 \mathrm{E}+00$ & $0.00000 \mathrm{E}+00$ & $0.00000 \mathrm{E}+00$ & 3.67013E-11 & $3.82968 \mathrm{E}-11$ & $82527 \mathrm{E}-11$ \\
\hline Cs-149 & $0.00000 \mathrm{E}+00$ & $0.00000 \mathrm{E}+00$ & $0.00000 \mathrm{E}+00$ & $5796 \mathrm{E}-13$ & $7.31244 \mathrm{E}-13$ & $33237 \mathrm{E}-13$ \\
\hline$C s-150$ & $0.00000 \mathrm{E}+00$ & $0.00000 \mathrm{E}+00$ & $0.00000 \mathrm{E}+00$ & $3856 \mathrm{E}-14$ & $1.73279 \mathrm{E}-14$ & $0 \mathrm{E}-14$ \\
\hline Cs-151 & $0.00000 \mathrm{E}+00$ & $0.00000 \mathrm{E}+00$ & $0.00000 \mathrm{E}+00$ & $5411 \mathrm{E}-15$ & $2.65916 \mathrm{E}-15$ & $0 \mathrm{E}-15$ \\
\hline$B a-129$ & $0.00000 \mathrm{E}+00$ & $0.00000 \mathrm{E}+00$ & $0.00000 \mathrm{E}+00$ & $1.77682 \mathrm{E}-30$ & $2.96392 \mathrm{E}-30$ & $36432 \mathrm{E}-30$ \\
\hline$B a-131$ & $0.00000 \mathrm{E}+00$ & $0.00000 \mathrm{E}+00$ & $0.00000 \mathrm{E}+00$ & $2.51675 \mathrm{E}-13$ & $3.53100 \mathrm{E}-13$ & $75670 \mathrm{E}-13$ \\
\hline$B a-135 m$ & $0.00000 \mathrm{E}+00$ & $0.00000 \mathrm{E}+00$ & $0.00000 \mathrm{E}+00$ & $1.80739 \mathrm{E}-06$ & $1.78940 \mathrm{E}-06$ & $45 \mathrm{E}-06$ \\
\hline$B a-136 m$ & $0.00000 \mathrm{E}+00$ & $0.00000 \mathrm{E}+00$ & $0.00000 \mathrm{E}+00$ & 4.16607E-08 & $4.22791 \mathrm{E}-08$ & 4.22767E-08 \\
\hline$B a-137 m$ & $0.00000 \mathrm{E}+00$ & $0.00000 \mathrm{E}+00$ & $0.00000 \mathrm{E}+00$ & $5.60485 \mathrm{E}-04$ & 5.60146E-04 & $5.60216 \mathrm{E}-04$ \\
\hline$B a-139$ & $0.00000 \mathrm{E}+00$ & $0.00000 \mathrm{E}+00$ & $0.00000 \mathrm{E}+00$ & $3.02198 \mathrm{E}-01$ & $3.02066 \mathrm{E}-01$ & $3.01956 \mathrm{E}-01$ \\
\hline$B a-141$ & $0.00000 \mathrm{E}+00$ & $0.00000 \mathrm{E}+00$ & $0.00000 \mathrm{E}+00$ & $6.11881 \mathrm{E}-02$ & $6.12169 \mathrm{E}-02$ & $11699 \mathrm{E}-02$ \\
\hline$B a-142$ & $0.00000 \mathrm{E}+00$ & $0.00000 \mathrm{E}+00$ & $0.00000 \mathrm{E}+00$ & $3.43204 \mathrm{E}-02$ & $3.43492 \mathrm{E}-02$ & 3.43384E-02 \\
\hline$B a-143$ & $0.00000 \mathrm{E}+00$ & $0.00000 \mathrm{E}+00$ & $0.00000 \mathrm{E}+00$ & $6.82208 \mathrm{E}-04$ & $6.82300 \mathrm{E}-04$ & $6.83309 \mathrm{E}-04$ \\
\hline$B a-144$ & $0.00000 \mathrm{E}+00$ & $0.00000 \mathrm{E}+00$ & $0.00000 \mathrm{E}+00$ & 4.21253E-04 & 4.21621E-04 & 4.22593E-04 \\
\hline$B a-145$ & $0.00000 \mathrm{E}+00$ & $0.00000 \mathrm{E}+00$ & $0.00000 \mathrm{E}+00$ & $6.83683 \mathrm{E}-05$ & $6.84125 \mathrm{E}-05$ & $6.86572 \mathrm{E}-05$ \\
\hline$B a-146$ & $0.00000 \mathrm{E}+00$ & $0.00000 \mathrm{E}+00$ & $0.00000 \mathrm{E}+00$ & $1.52197 \mathrm{E}-05$ & $1.52356 \mathrm{E}-05$ & $1.53234 \mathrm{E}-05$ \\
\hline$B a-147$ & $0.00000 \mathrm{E}+00$ & $0.00000 \mathrm{E}+00$ & $0.00000 \mathrm{E}+00$ & $1.54400 \mathrm{E}-06$ & $1.54496 \mathrm{E}-06$ & $1.55704 \mathrm{E}-06$ \\
\hline$B a-148$ & $0.00000 \mathrm{E}+00$ & $0.00000 \mathrm{E}+00$ & $0.00000 \mathrm{E}+00$ & $1.14207 \mathrm{E}-07$ & $1.14518 \mathrm{E}-07$ & $1.15341 \mathrm{E}-07$ \\
\hline$B a-149$ & $0.00000 \mathrm{E}+00$ & $0.00000 \mathrm{E}+00$ & $0.00000 \mathrm{E}+00$ & 4.41753E-09 & 4.46028E-09 & $.48051 \mathrm{E}-09$ \\
\hline$B a-150$ & $0.00000 \mathrm{E}+00$ & $0.00000 \mathrm{E}+00$ & $0.00000 \mathrm{E}+00$ & $2.43637 \mathrm{E}-10$ & $2.49379 \mathrm{E}-10$ & $.49826 \mathrm{E}-10$ \\
\hline$B a-151$ & $0.00000 \mathrm{E}+00$ & $0.00000 \mathrm{E}+00$ & $0.00000 \mathrm{E}+00$ & $9.48964 \mathrm{E}-12$ & $1.08128 \mathrm{E}-11$ & $1.06208 \mathrm{E}-11$ \\
\hline$B a-152$ & $0.00000 \mathrm{E}+00$ & $0.00000 \mathrm{E}+00$ & $0.00000 \mathrm{E}+00$ & $9.02724 \mathrm{E}-14$ & $9.66879 \mathrm{E}-14$ & $9.60356 \mathrm{E}-14$ \\
\hline$B a-153$ & $0.00000 \mathrm{E}+00$ & $0.00000 \mathrm{E}+00$ & $0.00000 \mathrm{E}+00$ & $1.48943 \mathrm{E}-15$ & $1.80012 \mathrm{E}-15$ & $1.75305 \mathrm{E}-15$ \\
\hline$L a-133$ & $0.00000 \mathrm{E}+00$ & $0.00000 \mathrm{E}+00$ & $0.00000 \mathrm{E}+00$ & $2.87183 \mathrm{E}-17$ & $2.91621 \mathrm{E}-17$ & $3.11300 \mathrm{E}-17$ \\
\hline$L a-135$ & $0.00000 \mathrm{E}+00$ & $0.00000 \mathrm{E}+00$ & $0.00000 \mathrm{E}+00$ & $7.04341 \mathrm{E}-11$ & $6.94627 \mathrm{E}-11$ & $6.81003 \mathrm{E}-11$ \\
\hline La-136 & $0.00000 \mathrm{E}+00$ & $0.00000 \mathrm{E}+00$ & $0.00000 \mathrm{E}+00$ & $0.00000 \mathrm{E}+00$ & $0.00000 \mathrm{E}+00$ & $0.00000 \mathrm{E}+00$ \\
\hline La-137 & $0.00000 \mathrm{E}+00$ & $0.00000 \mathrm{E}+00$ & $0.00000 \mathrm{E}+00$ & $2.93808 \mathrm{E}-04$ & $2.85644 \mathrm{E}-04$ & $3.00652 \mathrm{E}-04$ \\
\hline
\end{tabular}




\begin{tabular}{|c|c|c|c|c|c|c|}
\hline \multirow[b]{2}{*}{ Nuclide } & \multicolumn{3}{|c|}{ Fresh Fuel } & \multicolumn{3}{|c|}{ Discharge } \\
\hline & Unit Cell & $\begin{array}{l}\text { Full Core } \\
\text { (NO LBP) }\end{array}$ & Full Core (3) & Unit Cell & $\begin{array}{l}\text { Full Core } \\
\text { (NO LBP) }\end{array}$ & Full Core (3) \\
\hline$L a-141$ & $0.00000 \mathrm{E}+00$ & $0.00000 \mathrm{E}+00$ & $0.00000 \mathrm{E}+00$ & $7.95409 \mathrm{E}-01$ & $7.95838 \mathrm{E}-01$ & $7.95145 \mathrm{E}-01$ \\
\hline$L a-142$ & $0.00000 \mathrm{E}+00$ & $0.00000 \mathrm{E}+00$ & $0.00000 \mathrm{E}+00$ & $3.05725 \mathrm{E}-01$ & $3.06009 \mathrm{E}-01$ & $3.05806 \mathrm{E}-01$ \\
\hline La-143 & $0.00000 \mathrm{E}+00$ & $0.00000 \mathrm{E}+00$ & $0.00000 \mathrm{E}+00$ & $4.61069 \mathrm{E}-02$ & 4.61525E-02 & 4.61606E-02 \\
\hline$L a-144$ & $0.00000 \mathrm{E}+00$ & $0.00000 \mathrm{E}+00$ & $0.00000 \mathrm{E}+00$ & $1.95443 \mathrm{E}-03$ & $1.95573 \mathrm{E}-03$ & $1.95786 \mathrm{E}-03$ \\
\hline$L a-145$ & $0.00000 \mathrm{E}+00$ & $0.00000 \mathrm{E}+00$ & $0.00000 \mathrm{E}+00$ & $8.37344 \mathrm{E}-04$ & $8.37533 \mathrm{E}-04$ & 8.38673E-04 \\
\hline$L a-146$ & $0.00000 \mathrm{E}+00$ & $0.00000 \mathrm{E}+00$ & $0.00000 \mathrm{E}+00$ & $8.61787 \mathrm{E}-05$ & $8.61551 \mathrm{E}-05$ & 8.64417E-05 \\
\hline$L a-146 m$ & $0.00000 \mathrm{E}+00$ & $0.00000 \mathrm{E}+00$ & $0.00000 \mathrm{E}+00$ & $6.88905 \mathrm{E}-05$ & $6.87817 \mathrm{E}-05$ & $6.88436 \mathrm{E}-05$ \\
\hline$L a-147$ & $0.00000 \mathrm{E}+00$ & $0.00000 \mathrm{E}+00$ & $0.00000 \mathrm{E}+00$ & $3.52280 \mathrm{E}-05$ & $3.51262 \mathrm{E}-05$ & $3.51973 \mathrm{E}-05$ \\
\hline$L a-148$ & $0.00000 \mathrm{E}+00$ & $0.00000 \mathrm{E}+00$ & $0.00000 \mathrm{E}+00$ & $4.01918 \mathrm{E}-06$ & $4.00665 \mathrm{E}-06$ & 4.02977E-06 \\
\hline La-149 & $0.00000 \mathrm{E}+00$ & $0.00000 \mathrm{E}+00$ & $0.00000 \mathrm{E}+00$ & $8.74493 \mathrm{E}-07$ & $8.71352 \mathrm{E}-07$ & $75673 \mathrm{E}-07$ \\
\hline$L a-150$ & $0.00000 \mathrm{E}+00$ & $0.00000 \mathrm{E}+00$ & $0.00000 \mathrm{E}+00$ & $6.97095 \mathrm{E}-08$ & $6.93811 \mathrm{E}-08$ & $4 \mathrm{E}-08$ \\
\hline$L a-151$ & $0.00000 \mathrm{E}+00$ & $0.00000 \mathrm{E}+00$ & $0.00000 \mathrm{E}+00$ & $1.27593 \mathrm{E}-08$ & $1.27483 \mathrm{E}-08$ & $1.28105 \mathrm{E}-08$ \\
\hline$L a-152$ & $0.00000 \mathrm{E}+00$ & $0.00000 \mathrm{E}+00$ & $0.00000 \mathrm{E}+00$ & $5.79243 \mathrm{E}-10$ & $5.80489 \mathrm{E}-10$ & $5.83036 \mathrm{E}-10$ \\
\hline$L a-153$ & $0.00000 \mathrm{E}+00$ & $0.00000 \mathrm{E}+00$ & $0.00000 \mathrm{E}+00$ & $2.49666 \mathrm{E}-11$ & $2.55783 \mathrm{E}-11$ & $2.55929 \mathrm{E}-11$ \\
\hline La-154 & $0.00000 \mathrm{E}+00$ & $0.00000 \mathrm{E}+00$ & $0.00000 \mathrm{E}+00$ & $2.40690 \mathrm{E}-13$ & $2.49915 \mathrm{E}-13$ & $2.49439 \mathrm{E}-13$ \\
\hline$L a-155$ & $0.00000 \mathrm{E}+00$ & $0.00000 \mathrm{E}+00$ & $0.00000 \mathrm{E}+00$ & $6.41698 \mathrm{E}-15$ & $6.47517 \mathrm{E}-15$ & $.49740 \mathrm{E}-15$ \\
\hline $\mathrm{Ce}-135$ & $0.00000 \mathrm{E}+00$ & $0.00000 \mathrm{E}+00$ & $0.00000 \mathrm{E}+00$ & $7.49070 \mathrm{E}-17$ & 7.38416E-17 & $.91205 \mathrm{E}-17$ \\
\hline $\mathrm{Ce}-137$ & $0.00000 \mathrm{E}+00$ & $0.00000 \mathrm{E}+00$ & $0.00000 \mathrm{E}+00$ & $7.83365 \mathrm{E}-12$ & $7.72944 \mathrm{E}-12$ & 7.60074E-12 \\
\hline $\mathrm{Ce}-139 \mathrm{~m}$ & $0.00000 \mathrm{E}+00$ & $0.00000 \mathrm{E}+00$ & $0.00000 \mathrm{E}+00$ & $3.97619 \mathrm{E}-11$ & 3.94243E-11 & $3.86369 \mathrm{E}-11$ \\
\hline $\mathrm{Ce}-145$ & $0.00000 \mathrm{E}+00$ & $0.00000 \mathrm{E}+00$ & $0.00000 \mathrm{E}+00$ & $6.54654 \mathrm{E}-03$ & $6.54845 \mathrm{E}-03$ & $6.55062 \mathrm{E}-03$ \\
\hline $\mathrm{Ce}-146$ & $0.00000 \mathrm{E}+00$ & $0.00000 \mathrm{E}+00$ & $0.00000 \mathrm{E}+00$ & $2.29300 \mathrm{E}-02$ & $2.29203 \mathrm{E}-02$ & 2.29253E-02 \\
\hline $\mathrm{Ce}-147$ & $0.00000 \mathrm{E}+00$ & $0.00000 \mathrm{E}+00$ & $0.00000 \mathrm{E}+00$ & $1.08977 \mathrm{E}-03$ & $1.08770 \mathrm{E}-03$ & $1.08714 \mathrm{E}-03$ \\
\hline $\mathrm{Ce}-148$ & $0.00000 \mathrm{E}+00$ & $0.00000 \mathrm{E}+00$ & $0.00000 \mathrm{E}+00$ & $8.15521 \mathrm{E}-04$ & $8.14759 \mathrm{E}-04$ & $8.16363 \mathrm{E}-04$ \\
\hline $\mathrm{Ce}-149$ & $0.00000 \mathrm{E}+00$ & $0.00000 \mathrm{E}+00$ & $0.00000 \mathrm{E}+00$ & 4.08106E-05 & $4.06809 \mathrm{E}-05$ & $.07497 \mathrm{E}-05$ \\
\hline $\mathrm{Ce}-150$ & $0.00000 \mathrm{E}+00$ & $0.00000 \mathrm{E}+00$ & $0.00000 \mathrm{E}+00$ & $1.58438 \mathrm{E}-05$ & $1.57784 \mathrm{E}-05$ & 58204E-05 \\
\hline $\mathrm{Ce}-151$ & $0.00000 \mathrm{E}+00$ & $0.00000 \mathrm{E}+00$ & $0.00000 \mathrm{E}+00$ & $1.10518 \mathrm{E}-06$ & $1.09797 \mathrm{E}-06$ & $10248 \mathrm{E}-06$ \\
\hline $\mathrm{Ce}-152$ & $0.00000 \mathrm{E}+00$ & $0.00000 \mathrm{E}+00$ & $0.00000 \mathrm{E}+00$ & $3.52895 \mathrm{E}-07$ & 3.49935E-07 & $3.51776 \mathrm{E}-07$ \\
\hline $\mathrm{Ce}-153$ & $0.00000 \mathrm{E}+00$ & $0.00000 \mathrm{E}+00$ & $0.00000 \mathrm{E}+00$ & $3.11241 \mathrm{E}-08$ & $3.08159 \mathrm{E}-08$ & 3.09994E-08 \\
\hline $\mathrm{Ce}-154$ & $0.00000 \mathrm{E}+00$ & $0.00000 \mathrm{E}+00$ & $0.00000 \mathrm{E}+00$ & $2.28714 \mathrm{E}-09$ & $2.26455 \mathrm{E}-09$ & $2.27721 \mathrm{E}-09$ \\
\hline $\mathrm{Ce}-155$ & $0.00000 \mathrm{E}+00$ & $0.00000 \mathrm{E}+00$ & $0.00000 \mathrm{E}+00$ & $8.94359 \mathrm{E}-11$ & $8.91116 \mathrm{E}-11$ & $8.95385 \mathrm{E}-11$ \\
\hline $\mathrm{Ce}-156$ & $0.00000 \mathrm{E}+00$ & $0.00000 \mathrm{E}+00$ & $0.00000 \mathrm{E}+00$ & $1.46734 \mathrm{E}-12$ & $1.46920 \mathrm{E}-12$ & $1.47524 \mathrm{E}-12$ \\
\hline $\mathrm{Ce}-157$ & $0.00000 \mathrm{E}+00$ & $0.00000 \mathrm{E}+00$ & $0.00000 \mathrm{E}+00$ & $1.64169 \mathrm{E}-14$ & $1.65270 \mathrm{E}-14$ & $1.65859 \mathrm{E}-14$ \\
\hline Pr-139 & $0.00000 \mathrm{E}+00$ & $0.00000 \mathrm{E}+00$ & $0.00000 \mathrm{E}+00$ & $1.72093 \mathrm{E}-13$ & $1.69593 \mathrm{E}-13$ & $1.69596 \mathrm{E}-13$ \\
\hline $\operatorname{Pr}-140$ & $0.00000 \mathrm{E}+00$ & $0.00000 \mathrm{E}+00$ & $0.00000 \mathrm{E}+00$ & $6.73117 \mathrm{E}-09$ & 8.08744E-09 & $8.13250 \mathrm{E}-09$ \\
\hline Pr-142m & $0.00000 \mathrm{E}+00$ & $0.00000 \mathrm{E}+00$ & $0.00000 \mathrm{E}+00$ & $1.51906 \mathrm{E}-09$ & $1.50787 \mathrm{E}-09$ & $1.47814 \mathrm{E}-09$ \\
\hline $\operatorname{Pr}-144$ & $0.00000 \mathrm{E}+00$ & $0.00000 \mathrm{E}+00$ & $0.00000 \mathrm{E}+00$ & $5.02424 \mathrm{E}-02$ & $5.03563 \mathrm{E}-02$ & $5.01949 \mathrm{E}-02$ \\
\hline
\end{tabular}




\section{Depletion Analysis of Modular High Temperature Gas-cooled Reactor Loaded with LEU/Thorium Fuel}

February 2012

65

\begin{tabular}{|c|c|c|c|c|c|c|}
\hline \multirow[b]{2}{*}{ Nuclide } & \multicolumn{3}{|c|}{ Fresh Fuel } & \multicolumn{3}{|c|}{ Discharge } \\
\hline & Unit Cell & $\begin{array}{l}\text { Full Core } \\
\text { (NO LBP) }\end{array}$ & Full Core (3) & Unit Cell & $\begin{array}{l}\text { Full Core } \\
\text { (NO LBP) }\end{array}$ & Full Core (3) \\
\hline $\operatorname{Pr}-144 m$ & $0.00000 \mathrm{E}+00$ & $0.00000 \mathrm{E}+00$ & $0.00000 \mathrm{E}+00$ & $1.98310 \mathrm{E}-04$ & $1.98715 \mathrm{E}-04$ & $1.98122 \mathrm{E}-04$ \\
\hline $\operatorname{Pr}-145$ & $0.00000 \mathrm{E}+00$ & $0.00000 \mathrm{E}+00$ & $0.00000 \mathrm{E}+00$ & $7.81660 \mathrm{E}-01$ & $7.81888 \mathrm{E}-01$ & 7.82142E-01 \\
\hline $\operatorname{Pr}-146$ & $0.00000 \mathrm{E}+00$ & $0.00000 \mathrm{E}+00$ & $0.00000 \mathrm{E}+00$ & $4.11055 \mathrm{E}-02$ & $4.10883 \mathrm{E}-02$ & 4.10953E-02 \\
\hline $\operatorname{Pr}-147$ & $0.00000 \mathrm{E}+00$ & $0.00000 \mathrm{E}+00$ & $0.00000 \mathrm{E}+00$ & $1.74072 \mathrm{E}-02$ & $1.73796 \mathrm{E}-02$ & $1.73807 \mathrm{E}-02$ \\
\hline $\operatorname{Pr}-148$ & $0.00000 \mathrm{E}+00$ & $0.00000 \mathrm{E}+00$ & $0.00000 \mathrm{E}+00$ & $2.15396 \mathrm{E}-03$ & $2.15029 \mathrm{E}-03$ & $2.15196 \mathrm{E}-03$ \\
\hline $\operatorname{Pr}-148 m$ & $0.00000 \mathrm{E}+00$ & $0.00000 \mathrm{E}+00$ & $0.00000 \mathrm{E}+00$ & $1.34326 \mathrm{E}-04$ & $1.32757 \mathrm{E}-04$ & $1.30767 \mathrm{E}-04$ \\
\hline Pr-149 & $0.00000 \mathrm{E}+00$ & $0.00000 \mathrm{E}+00$ & $0.00000 \mathrm{E}+00$ & $1.52841 \mathrm{E}-03$ & $1.52262 \mathrm{E}-03$ & $1.52187 \mathrm{E}-03$ \\
\hline $\operatorname{Pr}-150$ & $0.00000 \mathrm{E}+00$ & $0.00000 \mathrm{E}+00$ & $0.00000 \mathrm{E}+00$ & $4.43588 \mathrm{E}-05$ & $4.41028 \mathrm{E}-05$ & $4.40646 \mathrm{E}-05$ \\
\hline $\operatorname{Pr}-151$ & $0.00000 \mathrm{E}+00$ & $0.00000 \mathrm{E}+00$ & $0.00000 \mathrm{E}+00$ & 7.69873E-05 & $7.64168 \mathrm{E}-05$ & 7.63990E-05 \\
\hline $\operatorname{Pr}-152$ & $0.00000 \mathrm{E}+00$ & $0.00000 \mathrm{E}+00$ & $0.00000 \mathrm{E}+00$ & $6.26067 \mathrm{E}-06$ & $6.19254 \mathrm{E}-06$ & $6.19758 \mathrm{E}-06$ \\
\hline $\operatorname{Pr}-153$ & $0.00000 \mathrm{E}+00$ & $0.00000 \mathrm{E}+00$ & $0.00000 \mathrm{E}+00$ & $2.60389 \mathrm{E}-06$ & $2.57016 \mathrm{E}-06$ & $.57673 \mathrm{E}-06$ \\
\hline $\operatorname{Pr}-154$ & $0.00000 \mathrm{E}+00$ & $0.00000 \mathrm{E}+00$ & $0.00000 \mathrm{E}+00$ & $3.11658 \mathrm{E}-07$ & $3.05789 \mathrm{E}-07$ & 06949E-07 \\
\hline $\operatorname{Pr}-155$ & $0.00000 \mathrm{E}+00$ & $0.00000 \mathrm{E}+00$ & $0.00000 \mathrm{E}+00$ & $2.15823 \mathrm{E}-08$ & $2.11747 \mathrm{E}-08$ & $.12763 \mathrm{E}-08$ \\
\hline $\operatorname{Pr}-156$ & $0.00000 \mathrm{E}+00$ & $0.00000 \mathrm{E}+00$ & $0.00000 \mathrm{E}+00$ & $2.61084 \mathrm{E}-09$ & $2.55416 \mathrm{E}-09$ & $.57070 \mathrm{E}-09$ \\
\hline $\operatorname{Pr}-157$ & $0.00000 \mathrm{E}+00$ & $0.00000 \mathrm{E}+00$ & $0.00000 \mathrm{E}+00$ & $1.11900 \mathrm{E}-10$ & $1.09481 \mathrm{E}-10$ & $.10323 \mathrm{E}-10$ \\
\hline $\operatorname{Pr}-158$ & $0.00000 \mathrm{E}+00$ & $0.00000 \mathrm{E}+00$ & $0.00000 \mathrm{E}+00$ & $4.65759 \mathrm{E}-12$ & $4.55430 \mathrm{E}-12$ & $59355 \mathrm{E}-12$ \\
\hline Pr-159 & $0.00000 \mathrm{E}+00$ & $0.00000 \mathrm{E}+00$ & $0.00000 \mathrm{E}+00$ & $936 \mathrm{E}-14$ & $263 \mathrm{E}-14$ & $70 \mathrm{E}-14$ \\
\hline$N d-140$ & $0.00000 \mathrm{E}+00$ & $0.00000 \mathrm{E}+00$ & $0.00000 \mathrm{E}+00$ & $1.75566 \mathrm{E}-15$ & $1.75218 \mathrm{E}-15$ & $1.87567 \mathrm{E}-15$ \\
\hline$N d-141$ & $0.00000 \mathrm{E}+00$ & $0.00000 \mathrm{E}+00$ & $0.00000 \mathrm{E}+00$ & $5.44326 \mathrm{E}-09$ & $6.68301 \mathrm{E}-09$ & $6.53679 \mathrm{E}-09$ \\
\hline$N d-149$ & $0.00000 \mathrm{E}+00$ & $0.00000 \mathrm{E}+00$ & $0.00000 \mathrm{E}+00$ & $7.33721 \mathrm{E}-02$ & 7.31972E-02 & $7.30680 \mathrm{E}-02$ \\
\hline$N d-151$ & $0.00000 \mathrm{E}+00$ & $0.00000 \mathrm{E}+00$ & $0.00000 \mathrm{E}+00$ & $4.17925 \mathrm{E}-03$ & $4.15245 \mathrm{E}-03$ & 4.13905E-03 \\
\hline Nd-152 & $0.00000 \mathrm{E}+00$ & $0.00000 \mathrm{E}+00$ & $0.00000 \mathrm{E}+00$ & $2.63868 \mathrm{E}-03$ & $2.61468 \mathrm{E}-03$ & $2.60598 \mathrm{E}-03$ \\
\hline$N d-153$ & $0.00000 \mathrm{E}+00$ & $0.00000 \mathrm{E}+00$ & $0.00000 \mathrm{E}+00$ & 7.05871E-05 & $6.98254 \mathrm{E}-05$ & $6.96889 \mathrm{E}-05$ \\
\hline$N d-154$ & $0.00000 \mathrm{E}+00$ & $0.00000 \mathrm{E}+00$ & $0.00000 \mathrm{E}+00$ & $2.98813 \mathrm{E}-05$ & $2.94562 \mathrm{E}-05$ & $2.93873 \mathrm{E}-05$ \\
\hline$N d-155$ & $0.00000 \mathrm{E}+00$ & $0.00000 \mathrm{E}+00$ & $0.00000 \mathrm{E}+00$ & $3.77137 \mathrm{E}-06$ & 3.70603E-06 & $3.70348 \mathrm{E}-06$ \\
\hline$N d-156$ & $0.00000 \mathrm{E}+00$ & $0.00000 \mathrm{E}+00$ & $0.00000 \mathrm{E}+00$ & $8.26186 \mathrm{E}-07$ & $8.09277 \mathrm{E}-07$ & $8.09948 \mathrm{E}-07$ \\
\hline$N d-157$ & $0.00000 \mathrm{E}+00$ & $0.00000 \mathrm{E}+00$ & $0.00000 \mathrm{E}+00$ & 6.82894E-08 & $6.64993 \mathrm{E}-08$ & $6.68493 \mathrm{E}-08$ \\
\hline$N d-158$ & $0.00000 \mathrm{E}+00$ & $0.00000 \mathrm{E}+00$ & $0.00000 \mathrm{E}+00$ & 8.40615E-09 & 8.16764E-09 & $.23138 \mathrm{E}-09$ \\
\hline$N d-159$ & $0.00000 \mathrm{E}+00$ & $0.00000 \mathrm{E}+00$ & $0.00000 \mathrm{E}+00$ & $4.17473 \mathrm{E}-10$ & 4.05357E-10 & 4.09007E-10 \\
\hline$N d-160$ & $0.00000 \mathrm{E}+00$ & $0.00000 \mathrm{E}+00$ & $0.00000 \mathrm{E}+00$ & $9.96213 \mathrm{E}-12$ & $9.69439 \mathrm{E}-12$ & $9.78156 \mathrm{E}-12$ \\
\hline$N d-161$ & $0.00000 \mathrm{E}+00$ & $0.00000 \mathrm{E}+00$ & $0.00000 \mathrm{E}+00$ & $2.40804 \mathrm{E}-13$ & $2.34697 \mathrm{E}-13$ & $2.36855 \mathrm{E}-13$ \\
\hline Pm-141 & $0.00000 \mathrm{E}+00$ & $0.00000 \mathrm{E}+00$ & $0.00000 \mathrm{E}+00$ & $0.00000 \mathrm{E}+00$ & $0.00000 \mathrm{E}+00$ & $0.00000 \mathrm{E}+00$ \\
\hline Pm-143 & $0.00000 \mathrm{E}+00$ & $0.00000 \mathrm{E}+00$ & $0.00000 \mathrm{E}+00$ & $4.83465 \mathrm{E}-13$ & $4.80981 \mathrm{E}-13$ & $5.27541 \mathrm{E}-13$ \\
\hline Pm-144 & $0.00000 \mathrm{E}+00$ & $0.00000 \mathrm{E}+00$ & $0.00000 \mathrm{E}+00$ & $8.55871 \mathrm{E}-11$ & $8.42606 \mathrm{E}-11$ & $8.86562 \mathrm{E}-11$ \\
\hline$P m-145$ & $0.00000 \mathrm{E}+00$ & $0.00000 \mathrm{E}+00$ & $0.00000 \mathrm{E}+00$ & $3.65110 \mathrm{E}-07$ & $4.21242 \mathrm{E}-07$ & 4.44133E-07 \\
\hline Pm-146 & $0.00000 \mathrm{E}+00$ & $0.00000 \mathrm{E}+00$ & $0.00000 \mathrm{E}+00$ & $1.29239 \mathrm{E}-03$ & $1.58122 \mathrm{E}-03$ & $1.63752 \mathrm{E}-03$ \\
\hline
\end{tabular}




\begin{tabular}{|c|c|c|c|c|c|c|}
\hline \multirow[b]{2}{*}{ Nuclide } & \multicolumn{3}{|c|}{ Fresh Fuel } & \multicolumn{3}{|c|}{ Discharge } \\
\hline & Unit Cell & $\begin{array}{l}\text { Full Core } \\
\text { (NO LBP) }\end{array}$ & Full Core (3) & Unit Cell & $\begin{array}{l}\text { Full Core } \\
\text { (NO LBP) }\end{array}$ & Full Core (3) \\
\hline$P m-150$ & $0.00000 \mathrm{E}+00$ & $0.00000 \mathrm{E}+00$ & $0.00000 \mathrm{E}+00$ & $2.92743 \mathrm{E}-03$ & $2.98531 \mathrm{E}-03$ & $2.84210 \mathrm{E}-03$ \\
\hline$P m-152$ & $0.00000 \mathrm{E}+00$ & $0.00000 \mathrm{E}+00$ & $0.00000 \mathrm{E}+00$ & $9.67943 \mathrm{E}-04$ & $9.59035 \mathrm{E}-04$ & $9.55561 \mathrm{E}-04$ \\
\hline$P m-152 m$ & $0.00000 \mathrm{E}+00$ & $0.00000 \mathrm{E}+00$ & $0.00000 \mathrm{E}+00$ & $1.72704 \mathrm{E}-05$ & $1.70463 \mathrm{E}-05$ & $1.67618 \mathrm{E}-05$ \\
\hline$P m-152 m$ & $0.00000 \mathrm{E}+00$ & $0.00000 \mathrm{E}+00$ & $0.00000 \mathrm{E}+00$ & $1.72704 \mathrm{E}-05$ & $1.70463 \mathrm{E}-05$ & $1.67618 \mathrm{E}-05$ \\
\hline Pm-153 & $0.00000 \mathrm{E}+00$ & $0.00000 \mathrm{E}+00$ & $0.00000 \mathrm{E}+00$ & 7.86136E-04 & $7.77501 \mathrm{E}-04$ & 7.75013E-04 \\
\hline Pm-154 & $0.00000 \mathrm{E}+00$ & $0.00000 \mathrm{E}+00$ & $0.00000 \mathrm{E}+00$ & $2.13902 \mathrm{E}-04$ & $2.10815 \mathrm{E}-04$ & $2.10036 \mathrm{E}-04$ \\
\hline$P m-154 m$ & $0.00000 \mathrm{E}+00$ & $0.00000 \mathrm{E}+00$ & $0.00000 \mathrm{E}+00$ & $1.83244 \mathrm{E}-05$ & $1.80354 \mathrm{E}-05$ & $1.78090 \mathrm{E}-05$ \\
\hline$P m-155$ & $0.00000 \mathrm{E}+00$ & $0.00000 \mathrm{E}+00$ & $0.00000 \mathrm{E}+00$ & $3.39978 \mathrm{E}-05$ & $3.34235 \mathrm{E}-05$ & $3.32410 \mathrm{E}-05$ \\
\hline$P m-156$ & $0.00000 \mathrm{E}+00$ & $0.00000 \mathrm{E}+00$ & $0.00000 \mathrm{E}+00$ & $1.19842 \mathrm{E}-05$ & $1.17445 \mathrm{E}-05$ & $1.16842 \mathrm{E}-05$ \\
\hline$P m-157$ & $0.00000 \mathrm{E}+00$ & $0.00000 \mathrm{E}+00$ & $0000 \mathrm{E}+00$ & $3650 \mathrm{E}-06$ & $2.18295 \mathrm{E}-06$ & $2 \mathrm{E}-06$ \\
\hline$P m-158$ & $0.00000 \mathrm{E}+00$ & $0.00000 \mathrm{E}+00$ & $0.00000 \mathrm{E}+00$ & $81 \mathrm{E}-07$ & $5858 \mathrm{E}-07$ & $3.46945 \mathrm{E}-07$ \\
\hline Pm-159 & $0.00000 \mathrm{E}+00$ & $0.00000 \mathrm{E}+00$ & $0.00000 \mathrm{E}+00$ & $2.66960 \mathrm{E}-08$ & $2.59155 \mathrm{E}-08$ & 2.60467E-08 \\
\hline$P m-160$ & $0.00000 \mathrm{E}+00$ & $0.00000 \mathrm{E}+00$ & $0.00000 \mathrm{E}+00$ & $5.47193 \mathrm{E}-09$ & 5.30607E-09 & 5.34365E-09 \\
\hline Pm-161 & $0.00000 \mathrm{E}+00$ & $0.00000 \mathrm{E}+00$ & $0.00000 \mathrm{E}+00$ & $2.08607 \mathrm{E}-10$ & $2.02296 \mathrm{E}-10$ & $2.03923 \mathrm{E}-10$ \\
\hline$P m-162$ & $0.00000 \mathrm{E}+00$ & $0.00000 \mathrm{E}+00$ & $0.00000 \mathrm{E}+00$ & $7.94656 \mathrm{E}-13$ & $8.15689 \mathrm{E}-13$ & $8.01563 \mathrm{E}-13$ \\
\hline$P m-163$ & $0.00000 \mathrm{E}+00$ & $0.00000 \mathrm{E}+00$ & $0.00000 \mathrm{E}+00$ & $1.37463 \mathrm{E}-14$ & $1.49120 \mathrm{E}-14$ & $1.46417 \mathrm{E}-14$ \\
\hline$S m-143$ & $0.00000 \mathrm{E}+00$ & $0.00000 \mathrm{E}+00$ & $0.00000 \mathrm{E}+00$ & $2.42942 \mathrm{E}-27$ & $2.76005 \mathrm{E}-27$ & $3.14540 \mathrm{E}-27$ \\
\hline$S m-143 m$ & $0.00000 \mathrm{E}+00$ & $0.00000 \mathrm{E}+00$ & $0.00000 \mathrm{E}+00$ & $0.00000 \mathrm{E}+00$ & $0.00000 \mathrm{E}+00$ & $0.00000 \mathrm{E}+00$ \\
\hline$S m-145$ & $0.00000 \mathrm{E}+00$ & $0.00000 \mathrm{E}+00$ & $0.00000 \mathrm{E}+00$ & $7.57279 \mathrm{E}-14$ & $7.55091 \mathrm{E}-14$ & $29648 \mathrm{E}-14$ \\
\hline$S m-146$ & $0.00000 \mathrm{E}+00$ & $0.00000 \mathrm{E}+00$ & $0.00000 \mathrm{E}+00$ & 4.87137E-04 & 5.94497E-04 & $02761 \mathrm{E}-04$ \\
\hline$S m-155$ & $0.00000 \mathrm{E}+00$ & $0.00000 \mathrm{E}+00$ & $0.00000 \mathrm{E}+00$ & $1.27172 \mathrm{E}-03$ & $1.25378 \mathrm{E}-03$ & $24542 \mathrm{E}-03$ \\
\hline$S m-156$ & $0.00000 \mathrm{E}+00$ & $0.00000 \mathrm{E}+00$ & $0.00000 \mathrm{E}+00$ & $3207 \mathrm{E}-02$ & $1.99342 \mathrm{E}-02$ & $6 \mathrm{E}-02$ \\
\hline Sm-157 & $0.00000 \mathrm{E}+00$ & $0.00000 \mathrm{E}+00$ & $0.00000 \mathrm{E}+00$ & $1.78150 \mathrm{E}-04$ & $1.74273 \mathrm{E}-04$ & $.73115 \mathrm{E}-04$ \\
\hline Sm-158 & $0.00000 \mathrm{E}+00$ & $0.00000 \mathrm{E}+00$ & $0.00000 \mathrm{E}+00$ & $6.69120 \mathrm{E}-05$ & $6.53264 \mathrm{E}-05$ & $50329 \mathrm{E}-05$ \\
\hline$S m-159$ & $0.00000 \mathrm{E}+00$ & $0.00000 \mathrm{E}+00$ & $0.00000 \mathrm{E}+00$ & $1.00391 \mathrm{E}-06$ & $9.78300 \mathrm{E}-07$ & $3 \mathrm{E}-07$ \\
\hline$S m-160$ & $0.00000 \mathrm{E}+00$ & $0.00000 \mathrm{E}+00$ & $0.00000 \mathrm{E}+00$ & $2.84420 \mathrm{E}-07$ & $2.76910 \mathrm{E}-07$ & 76524E-07 \\
\hline$S m-161$ & $0.00000 \mathrm{E}+00$ & $0.00000 \mathrm{E}+00$ & $0.00000 \mathrm{E}+00$ & $3.49110 \mathrm{E}-08$ & 3.39253E-08 & 39772E-08 \\
\hline$S m-162$ & $0.00000 \mathrm{E}+00$ & $0.00000 \mathrm{E}+00$ & $0.00000 \mathrm{E}+00$ & $6.68628 \mathrm{E}-10$ & $6.61272 \mathrm{E}-10$ & $6.48843 \mathrm{E}-10$ \\
\hline$S m-163$ & $0.00000 \mathrm{E}+00$ & $0.00000 \mathrm{E}+00$ & $0.00000 \mathrm{E}+00$ & & $2.59980 \mathrm{E}-11$ & $2.55780 \mathrm{E}-11$ \\
\hline$S m-164$ & $0.00000 \mathrm{E}+00$ & $0.00000 \mathrm{E}+00$ & $0.00000 \mathrm{E}+00$ & $8.31681 \mathrm{E}-13$ & $8.60742 \mathrm{E}-13$ & $8.46890 \mathrm{E}-13$ \\
\hline$S m-165$ & $0.00000 \mathrm{E}+00$ & $0.00000 \mathrm{E}+00$ & $0.00000 \mathrm{E}+00$ & $1.71332 \mathrm{E}-14$ & $1.88347 \mathrm{E}-14$ & $1.84645 \mathrm{E}-14$ \\
\hline$E u-147$ & $0.00000 \mathrm{E}+00$ & $0.00000 \mathrm{E}+00$ & $0.00000 \mathrm{E}+00$ & $1.34122 \mathrm{E}-15$ & $1.32014 \mathrm{E}-15$ & $1.42167 \mathrm{E}-15$ \\
\hline$E u-149$ & $0.00000 \mathrm{E}+00$ & $0.00000 \mathrm{E}+00$ & $0.00000 \mathrm{E}+00$ & $1.34540 \mathrm{E}-11$ & $1.35938 \mathrm{E}-11$ & $1.46944 \mathrm{E}-11$ \\
\hline$E u-150$ & $0.00000 \mathrm{E}+00$ & $0.00000 \mathrm{E}+00$ & $0.00000 \mathrm{E}+00$ & $4.20979 \mathrm{E}-08$ & 5.00124E-08 & $5.80856 \mathrm{E}-08$ \\
\hline$E u-152 m$ & $0.00000 \mathrm{E}+00$ & $0.00000 \mathrm{E}+00$ & $0.00000 \mathrm{E}+00$ & 4.15737E-09 & 4.08809E-09 & 4.00481E-09 \\
\hline$E u-152 m$ & $0.00000 \mathrm{E}+00$ & $0.00000 \mathrm{E}+00$ & $0.00000 \mathrm{E}+00$ & 4.15737E-09 & 4.08809E-09 & 4.00481E-09 \\
\hline
\end{tabular}




\section{Depletion Analysis of Modular High Temperature Gas-cooled Reactor Loaded with LEU/Thorium Fuel}

February 2012

\begin{tabular}{|c|c|c|c|c|c|c|}
\hline \multirow[b]{2}{*}{ Nuclide } & \multicolumn{3}{|c|}{ Fresh Fuel } & \multicolumn{3}{|c|}{ Discharge } \\
\hline & Unit Cell & $\begin{array}{l}\text { Full Core } \\
\text { (NO LBP) }\end{array}$ & Full Core (3) & Unit Cell & $\begin{array}{l}\text { Full Core } \\
\text { (NO LBP) }\end{array}$ & Full Core (3) \\
\hline $\mathrm{Eu}-154 \mathrm{~m}$ & $0.00000 \mathrm{E}+00$ & $0.00000 \mathrm{E}+00$ & $0.00000 \mathrm{E}+00$ & $1.06129 \mathrm{E}-07$ & $1.04256 \mathrm{E}-07$ & $1.01951 \mathrm{E}-07$ \\
\hline Eu-158 & $0.00000 \mathrm{E}+00$ & $0.00000 \mathrm{E}+00$ & $0.00000 \mathrm{E}+00$ & $6.27504 \mathrm{E}-04$ & $6.12827 \mathrm{E}-04$ & $6.09404 \mathrm{E}-04$ \\
\hline$E u-159$ & $0.00000 \mathrm{E}+00$ & $0.00000 \mathrm{E}+00$ & $0.00000 \mathrm{E}+00$ & $1.21207 \mathrm{E}-04$ & $1.18234 \mathrm{E}-04$ & $1.17595 \mathrm{E}-04$ \\
\hline$E u-160$ & $0.00000 \mathrm{E}+00$ & $0.00000 \mathrm{E}+00$ & $0.00000 \mathrm{E}+00$ & $1.79871 \mathrm{E}-06$ & $1.75354 \mathrm{E}-06$ & $1.74408 \mathrm{E}-06$ \\
\hline$E u-161$ & $0.00000 \mathrm{E}+00$ & $0.00000 \mathrm{E}+00$ & $0.00000 \mathrm{E}+00$ & 4.93345E-07 & 4.80731E-07 & 4.78069E-07 \\
\hline$E u-162$ & $0.00000 \mathrm{E}+00$ & $0.00000 \mathrm{E}+00$ & $0.00000 \mathrm{E}+00$ & $2.94186 \mathrm{E}-08$ & $2.89128 \mathrm{E}-08$ & $2.83499 \mathrm{E}-08$ \\
\hline$E u-163$ & $0.00000 \mathrm{E}+00$ & $0.00000 \mathrm{E}+00$ & $0.00000 \mathrm{E}+00$ & 3.78462E-09 & $3.72424 \mathrm{E}-09$ & 3.65977E-09 \\
\hline$E u-164$ & $0.00000 \mathrm{E}+00$ & $0.00000 \mathrm{E}+00$ & $0.00000 \mathrm{E}+00$ & $1.59802 \mathrm{E}-10$ & $1.58611 \mathrm{E}-10$ & $1.56175 \mathrm{E}-10$ \\
\hline$E u-165$ & $0.00000 \mathrm{E}+00$ & $0.00000 \mathrm{E}+00$ & $0.00000 \mathrm{E}+00$ & $1.03246 \mathrm{E}-11$ & $1.03942 \mathrm{E}-11$ & $2323 \mathrm{E}-11$ \\
\hline$E u-166$ & $0.00000 \mathrm{E}+00$ & $0.00000 \mathrm{E}+00$ & $0.00000 \mathrm{E}+00$ & $3.93125 \mathrm{E}-13$ & 4.09853E-13 & 02587E-13 \\
\hline$E u-167$ & $0.00000 \mathrm{E}+00$ & $0.00000 \mathrm{E}+00$ & $0.00000 \mathrm{E}+00$ & $1.54992 \mathrm{E}-14$ & $1.78916 \mathrm{E}-14$ & $1.75002 \mathrm{E}-14$ \\
\hline$G d-147$ & $0.00000 \mathrm{E}+00$ & $0.00000 \mathrm{E}+00$ & $0.00000 \mathrm{E}+00$ & $0.00000 \mathrm{E}+00$ & $0.00000 \mathrm{E}+00$ & $0.00000 \mathrm{E}+00$ \\
\hline$G d-149$ & $0.00000 \mathrm{E}+00$ & $0.00000 \mathrm{E}+00$ & $0.00000 \mathrm{E}+00$ & 5.67867E-17 & $5.59424 \mathrm{E}-17$ & $.00595 \mathrm{E}-17$ \\
\hline$G d-151$ & $0.00000 \mathrm{E}+00$ & $0.00000 \mathrm{E}+00$ & $0.00000 \mathrm{E}+00$ & $1.74535 \mathrm{E}-10$ & $2.00144 \mathrm{E}-10$ & $2.27277 \mathrm{E}-10$ \\
\hline$G d-153 m$ & $0.00000 \mathrm{E}+00$ & $0.00000 \mathrm{E}+00$ & $0.00000 \mathrm{E}+00$ & $2.44454 \mathrm{E}-27$ & $2.41849 \mathrm{E}-27$ & $.59114 \mathrm{E}-27$ \\
\hline$G d-159$ & $0.00000 \mathrm{E}+00$ & $0.00000 \mathrm{E}+00$ & $0.00000 \mathrm{E}+00$ & $9.05165 \mathrm{E}-03$ & $8.87865 \mathrm{E}-03$ & $.83124 \mathrm{E}-03$ \\
\hline$G d-161$ & $0.00000 \mathrm{E}+00$ & $0.00000 \mathrm{E}+00$ & $0.00000 \mathrm{E}+00$ & $5.07083 \mathrm{E}-06$ & $4.94828 \mathrm{E}-06$ & $.91160 \mathrm{E}-06$ \\
\hline$G d-162$ & $0.00000 \mathrm{E}+00$ & $0.00000 \mathrm{E}+00$ & $0.00000 \mathrm{E}+00$ & 3.06919E-06 & $3.01331 \mathrm{E}-06$ & $2.95253 \mathrm{E}-06$ \\
\hline$G d-163$ & $0.00000 \mathrm{E}+00$ & $0.00000 \mathrm{E}+00$ & $0.00000 \mathrm{E}+00$ & $1.58703 \mathrm{E}-07$ & $1.55841 \mathrm{E}-07$ & $1.52984 \mathrm{E}-07$ \\
\hline$G d-164$ & $0.00000 \mathrm{E}+00$ & $0.00000 \mathrm{E}+00$ & $0.00000 \mathrm{E}+00$ & 3.28072E-08 & $3.23262 \mathrm{E}-08$ & $3.17588 \mathrm{E}-08$ \\
\hline$G d-165$ & $0.00000 \mathrm{E}+00$ & $0.00000 \mathrm{E}+00$ & $0.00000 \mathrm{E}+00$ & $1.96060 \mathrm{E}-09$ & $1.93842 \mathrm{E}-09$ & $1.90568 \mathrm{E}-09$ \\
\hline$G d-166$ & $0.00000 \mathrm{E}+00$ & $0.00000 \mathrm{E}+00$ & $0.00000 \mathrm{E}+00$ & $3.52369 \mathrm{E}-10$ & $3.50337 \mathrm{E}-10$ & $3.43968 \mathrm{E}-10$ \\
\hline$G d-167$ & $0.00000 \mathrm{E}+00$ & $0.00000 \mathrm{E}+00$ & $0.00000 \mathrm{E}+00$ & $1.35560 \mathrm{E}-11$ & $1.40002 \mathrm{E}-11$ & $1.38103 \mathrm{E}-11$ \\
\hline$G d-168$ & $0.00000 \mathrm{E}+00$ & $0.00000 \mathrm{E}+00$ & $0.00000 \mathrm{E}+00$ & $6.52231 \mathrm{E}-13$ & $6.51178 \mathrm{E}-13$ & $6.54365 \mathrm{E}-13$ \\
\hline$G d-169$ & $0.00000 \mathrm{E}+00$ & $0.00000 \mathrm{E}+00$ & $0.00000 \mathrm{E}+00$ & $2.63992 \mathrm{E}-13$ & $2.69824 \mathrm{E}-13$ & $2.70247 \mathrm{E}-13$ \\
\hline$T b-151$ & $0.00000 \mathrm{E}+00$ & $0.00000 \mathrm{E}+00$ & $0.00000 \mathrm{E}+00$ & $0.00000 \mathrm{E}+00$ & $0.00000 \mathrm{E}+00$ & $0.00000 \mathrm{E}+00$ \\
\hline$T b-153$ & $0.00000 \mathrm{E}+00$ & $0.00000 \mathrm{E}+00$ & $0.00000 \mathrm{E}+00$ & 4.32339E-15 & $4.27731 \mathrm{E}-15$ & 4.58264E-15 \\
\hline$T b-155$ & $0.00000 \mathrm{E}+00$ & $0.00000 \mathrm{E}+00$ & $0.00000 \mathrm{E}+00$ & $1.12523 \mathrm{E}-11$ & $1.11291 \mathrm{E}-11$ & $.14067 \mathrm{E}-11$ \\
\hline$T b-156$ & $0.00000 \mathrm{E}+00$ & $0.00000 \mathrm{E}+00$ & $0.00000 \mathrm{E}+00$ & 4.30666E-10 & $4.23987 \mathrm{E}-10$ & $.21143 \mathrm{E}-10$ \\
\hline$T b-156 m$ & $0.00000 \mathrm{E}+00$ & $0.00000 \mathrm{E}+00$ & $0.00000 \mathrm{E}+00$ & $7.91731 \mathrm{E}-12$ & $7.78858 \mathrm{E}-12$ & $7.72447 \mathrm{E}-12$ \\
\hline$T b-157$ & $0.00000 \mathrm{E}+00$ & $0.00000 \mathrm{E}+00$ & $0.00000 \mathrm{E}+00$ & 8.89714E-07 & $8.62191 \mathrm{E}-07$ & $9.11686 \mathrm{E}-07$ \\
\hline$T b-158$ & $0.00000 \mathrm{E}+00$ & $0.00000 \mathrm{E}+00$ & $0.00000 \mathrm{E}+00$ & $1.65473 \mathrm{E}-05$ & $1.69237 \mathrm{E}-05$ & $1.81845 \mathrm{E}-05$ \\
\hline Tb-158m & $0.00000 \mathrm{E}+00$ & $0.00000 \mathrm{E}+00$ & $0.00000 \mathrm{E}+00$ & $3.31050 \mathrm{E}-13$ & $3.25024 \mathrm{E}-13$ & $3.18767 \mathrm{E}-13$ \\
\hline$T b-161$ & $0.00000 \mathrm{E}+00$ & $0.00000 \mathrm{E}+00$ & $0.00000 \mathrm{E}+00$ & $1.64378 \mathrm{E}-02$ & $1.60223 \mathrm{E}-02$ & $1.59334 \mathrm{E}-02$ \\
\hline$T b-162$ & $0.00000 \mathrm{E}+00$ & $0.00000 \mathrm{E}+00$ & $0.00000 \mathrm{E}+00$ & 2.81643E-06 & $2.76509 \mathrm{E}-06$ & 2.70933E-06 \\
\hline$T b-163$ & $0.00000 \mathrm{E}+00$ & $0.00000 \mathrm{E}+00$ & $0.00000 \mathrm{E}+00$ & 3.06524E-06 & $3.00941 \mathrm{E}-06$ & $2.95429 \mathrm{E}-06$ \\
\hline
\end{tabular}




\begin{tabular}{|c|c|c|c|c|c|c|}
\hline \multirow[b]{2}{*}{ Nuclide } & \multicolumn{3}{|c|}{ Fresh Fuel } & \multicolumn{3}{|c|}{ Discharge } \\
\hline & Unit Cell & $\begin{array}{l}\text { Full Core } \\
\text { (NO LBP) }\end{array}$ & Full Core (3) & Unit Cell & $\begin{array}{l}\text { Full Core } \\
(N O L B P)\end{array}$ & Full Core (3) \\
\hline$T b-164$ & $0.00000 \mathrm{E}+00$ & $0.00000 \mathrm{E}+00$ & $0.00000 \mathrm{E}+00$ & $1.71766 \mathrm{E}-07$ & $1.69125 \mathrm{E}-07$ & $1.66185 \mathrm{E}-07$ \\
\hline$T b-165$ & $0.00000 \mathrm{E}+00$ & $0.00000 \mathrm{E}+00$ & $0.00000 \mathrm{E}+00$ & $4.77072 \mathrm{E}-08$ & 4.70521E-08 & 4.62552E-08 \\
\hline$T b-166$ & $0.00000 \mathrm{E}+00$ & $0.00000 \mathrm{E}+00$ & $0.00000 \mathrm{E}+00$ & $3.28359 \mathrm{E}-09$ & $3.24769 \mathrm{E}-09$ & $3.18892 \mathrm{E}-09$ \\
\hline$T b-167$ & $0.00000 \mathrm{E}+00$ & $0.00000 \mathrm{E}+00$ & $0.00000 \mathrm{E}+00$ & $5.75738 \mathrm{E}-10$ & $5.76985 \mathrm{E}-10$ & $5.69809 \mathrm{E}-10$ \\
\hline$T b-168$ & $0.00000 \mathrm{E}+00$ & $0.00000 \mathrm{E}+00$ & $0.00000 \mathrm{E}+00$ & $1.05092 \mathrm{E}-10$ & $1.03683 \mathrm{E}-10$ & $1.04281 \mathrm{E}-10$ \\
\hline$T b-169$ & $0.00000 \mathrm{E}+00$ & $0.00000 \mathrm{E}+00$ & $0.00000 \mathrm{E}+00$ & $6.47970 \mathrm{E}-12$ & $6.45677 \mathrm{E}-12$ & $49681 \mathrm{E}-12$ \\
\hline$T b-170$ & $0.00000 \mathrm{E}+00$ & $0.00000 \mathrm{E}+00$ & $0.00000 \mathrm{E}+00$ & $1.76699 \mathrm{E}-12$ & $1.79006 \mathrm{E}-12$ & $9955 \mathrm{E}-12$ \\
\hline$T b-171$ & $0.00000 \mathrm{E}+00$ & $0.00000 \mathrm{E}+00$ & $0.00000 \mathrm{E}+00$ & $2.87722 \mathrm{E}-14$ & 3.18774E-14 & $5813 \mathrm{E}-14$ \\
\hline$D y-155$ & $0.00000 \mathrm{E}+00$ & $0.00000 \mathrm{E}+00$ & $0.00000 \mathrm{E}+00$ & $3.97700 \mathrm{E}-17$ & $3.91976 \mathrm{E}-17$ & $69 \mathrm{E}-17$ \\
\hline$D y-157$ & $0.00000 \mathrm{E}+00$ & $0.00000 \mathrm{E}+00$ & $0.00000 \mathrm{E}+00$ & $6139 \mathrm{E}-14$ & $2.74821 \mathrm{E}-14$ & $2 \mathrm{E}-14$ \\
\hline$D y-159$ & $0.00000 \mathrm{E}+00$ & $0.00000 \mathrm{E}+00$ & $0.00000 \mathrm{E}+00$ & $8 \mathrm{E}-08$ & $8.38657 \mathrm{E}-08$ & $9 \mathrm{E}-08$ \\
\hline$D y-165$ & $0.00000 \mathrm{E}+00$ & $0.00000 \mathrm{E}+00$ & $0.00000 \mathrm{E}+00$ & $5 \mathrm{E}-05$ & $4.32578 \mathrm{E}-05$ & 4.42589E-05 \\
\hline$D y-165 m$ & $0.00000 \mathrm{E}+00$ & $0.00000 \mathrm{E}+00$ & $0.00000 \mathrm{E}+00$ & $2.01137 \mathrm{E}-08$ & $1.98371 \mathrm{E}-08$ & $1.95011 \mathrm{E}-08$ \\
\hline$D y-166$ & $0.00000 \mathrm{E}+00$ & $0.00000 \mathrm{E}+00$ & $0.00000 \mathrm{E}+00$ & $5.89501 \mathrm{E}-05$ & $5.82880 \mathrm{E}-05$ & $5.72357 \mathrm{E}-05$ \\
\hline$D y-167$ & $0.00000 \mathrm{E}+00$ & $0.00000 \mathrm{E}+00$ & $0.00000 \mathrm{E}+00$ & $1.88410 \mathrm{E}-08$ & $1.88204 \mathrm{E}-08$ & $1.85761 \mathrm{E}-08$ \\
\hline$D y-168$ & $0.00000 \mathrm{E}+00$ & $0.00000 \mathrm{E}+00$ & $0.00000 \mathrm{E}+00$ & $1.32788 \mathrm{E}-08$ & $1.31010 \mathrm{E}-08$ & $1.31324 \mathrm{E}-08$ \\
\hline$D y-169$ & $0.00000 \mathrm{E}+00$ & $0.00000 \mathrm{E}+00$ & $0.00000 \mathrm{E}+00$ & $3.60945 \mathrm{E}-10$ & $3.57042 \mathrm{E}-10$ & $3.59164 \mathrm{E}-10$ \\
\hline$D y-170$ & $0.00000 \mathrm{E}+00$ & $0.00000 \mathrm{E}+00$ & $0.00000 \mathrm{E}+00$ & $8.33176 \mathrm{E}-11$ & $8.28272 \mathrm{E}-11$ & $.38013 \mathrm{E}-11$ \\
\hline$D y-171$ & $0.00000 \mathrm{E}+00$ & $0.00000 \mathrm{E}+00$ & $0.00000 \mathrm{E}+00$ & $3.15526 \mathrm{E}-12$ & $3.25937 \mathrm{E}-12$ & $98 \mathrm{E}-12$ \\
\hline$D y-172$ & $0.00000 \mathrm{E}+00$ & $0.00000 \mathrm{E}+00$ & $0.00000 \mathrm{E}+00$ & $7.04806 \mathrm{E}-13$ & $8.15405 \mathrm{E}-13$ & $.03238 \mathrm{E}-13$ \\
\hline Ho- 159 & $0.00000 \mathrm{E}+00$ & $0.00000 \mathrm{E}+00$ & $0.00000 \mathrm{E}+00$ & $9.10000 \mathrm{E}-17$ & $9.01366 \mathrm{E}-17$ & $65184 \mathrm{E}-17$ \\
\hline Ho- $159 \mathrm{~m}$ & $0.00000 \mathrm{E}+00$ & $0.00000 \mathrm{E}+00$ & $0.00000 \mathrm{E}+00$ & $4.58348 \mathrm{E}-20$ & $4.54258 \mathrm{E}-20$ & $.86418 \mathrm{E}-20$ \\
\hline Ho- 160 & $0.00000 \mathrm{E}+00$ & $0.00000 \mathrm{E}+00$ & $0.00000 \mathrm{E}+00$ & $4.63166 \mathrm{E}-30$ & $5.46763 \mathrm{E}-30$ & $.05075 \mathrm{E}-30$ \\
\hline Ho-161 & $0.00000 \mathrm{E}+00$ & $0.00000 \mathrm{E}+00$ & $0.00000 \mathrm{E}+00$ & $1.77285 \mathrm{E}-13$ & $1.75265 \mathrm{E}-13$ & $1.80837 \mathrm{E}-13$ \\
\hline Ho- $161 \mathrm{~m}$ & $0.00000 \mathrm{E}+00$ & $0.00000 \mathrm{E}+00$ & $0.00000 \mathrm{E}+00$ & $9.86900 \mathrm{E}-18$ & $9.83001 \mathrm{E}-18$ & $1.05101 \mathrm{E}-17$ \\
\hline Ho-162 & $0.00000 \mathrm{E}+00$ & $0.00000 \mathrm{E}+00$ & $0.00000 \mathrm{E}+00$ & $2.02199 \mathrm{E}-13$ & $1.99230 \mathrm{E}-13$ & $2.01303 \mathrm{E}-13$ \\
\hline Ho- $162 \mathrm{~m}$ & $0.00000 \mathrm{E}+00$ & $0.00000 \mathrm{E}+00$ & $0.00000 \mathrm{E}+00$ & $8.69333 \mathrm{E}-13$ & $8.56782 \mathrm{E}-13$ & $8.66039 \mathrm{E}-13$ \\
\hline Ho- 163 & $0.00000 \mathrm{E}+00$ & $0.00000 \mathrm{E}+00$ & $0.00000 \mathrm{E}+00$ & $1.33455 \mathrm{E}-07$ & $1.29776 \mathrm{E}-07$ & $1.38025 \mathrm{E}-07$ \\
\hline Ho- $163 \mathrm{~m}$ & $0.00000 \mathrm{E}+00$ & $0.00000 \mathrm{E}+00$ & $0.00000 \mathrm{E}+00$ & $5.55297 \mathrm{E}-16$ & $5.46309 \mathrm{E}-16$ & $5.46482 \mathrm{E}-16$ \\
\hline Ho- 164 & $0.00000 \mathrm{E}+00$ & $0.00000 \mathrm{E}+00$ & $0.00000 \mathrm{E}+00$ & $5.21690 \mathrm{E}-11$ & $5.33515 \mathrm{E}-11$ & $5.40955 \mathrm{E}-11$ \\
\hline Ho- $164 \mathrm{~m}$ & $0.00000 \mathrm{E}+00$ & $0.00000 \mathrm{E}+00$ & $0.00000 \mathrm{E}+00$ & 3.95457E-11 & $3.90540 \mathrm{E}-11$ & $3.89422 \mathrm{E}-11$ \\
\hline Ho- 166 & $0.00000 \mathrm{E}+00$ & $0.00000 \mathrm{E}+00$ & $0.00000 \mathrm{E}+00$ & $1.02643 \mathrm{E}-04$ & $9.93898 \mathrm{E}-05$ & $1.01802 \mathrm{E}-04$ \\
\hline Ho- 167 & $0.00000 \mathrm{E}+00$ & $0.00000 \mathrm{E}+00$ & $0.00000 \mathrm{E}+00$ & $5.81268 \mathrm{E}-07$ & $5.80588 \mathrm{E}-07$ & $5.73085 \mathrm{E}-07$ \\
\hline Ho- 168 & $0.00000 \mathrm{E}+00$ & $0.00000 \mathrm{E}+00$ & $0.00000 \mathrm{E}+00$ & $4.76702 \mathrm{E}-09$ & $4.70380 \mathrm{E}-09$ & 4.71519E-09 \\
\hline Ho-169 & $0.00000 \mathrm{E}+00$ & $0.00000 \mathrm{E}+00$ & $0.00000 \mathrm{E}+00$ & $2.99764 \mathrm{E}-09$ & $2.96403 \mathrm{E}-09$ & 2.98239E-09 \\
\hline Ho- 170 & $0.00000 \mathrm{E}+00$ & $0.00000 \mathrm{E}+00$ & $0.00000 \mathrm{E}+00$ & $5.23655 \mathrm{E}-10$ & 5.19613E-10 & $5.26774 \mathrm{E}-10$ \\
\hline
\end{tabular}




\section{Depletion Analysis of Modular High Temperature Gas-cooled Reactor Loaded with LEU/Thorium Fuel}

February 2012

\begin{tabular}{|c|c|c|c|c|c|c|}
\hline \multirow[b]{2}{*}{ Nuclide } & \multicolumn{3}{|c|}{ Fresh Fuel } & \multicolumn{3}{|c|}{ Discharge } \\
\hline & Unit Cell & $\begin{array}{l}\text { Full Core } \\
(N O L B P)\end{array}$ & Full Core (3) & Unit Cell & $\begin{array}{l}\text { Full Core } \\
\text { (NO LBP) }\end{array}$ & Full Core (3) \\
\hline $\mathrm{Ho}-170 \mathrm{~m}$ & $0.00000 \mathrm{E}+00$ & $0.00000 \mathrm{E}+00$ & $0.00000 \mathrm{E}+00$ & $1.65533 \mathrm{E}-11$ & $1.62068 \mathrm{E}-11$ & $1.66698 \mathrm{E}-11$ \\
\hline Ho- 171 & $0.00000 \mathrm{E}+00$ & $0.00000 \mathrm{E}+00$ & $0.00000 \mathrm{E}+00$ & $5.49601 \mathrm{E}-11$ & $5.53303 \mathrm{E}-11$ & $5.64752 \mathrm{E}-11$ \\
\hline Ho- 172 & $0.00000 \mathrm{E}+00$ & $0.00000 \mathrm{E}+00$ & $0.00000 \mathrm{E}+00$ & $1.23054 \mathrm{E}-11$ & $1.35146 \mathrm{E}-11$ & $1.35581 \mathrm{E}-11$ \\
\hline Er-161 & $0.00000 \mathrm{E}+00$ & $0.00000 \mathrm{E}+00$ & $0.00000 \mathrm{E}+00$ & $1.80966 \mathrm{E}-17$ & $1.78403 \mathrm{E}-17$ & $1.91128 \mathrm{E}-17$ \\
\hline$E r-163$ & $0.00000 \mathrm{E}+00$ & $0.00000 \mathrm{E}+00$ & $0.00000 \mathrm{E}+00$ & $1.98875 \mathrm{E}-15$ & $2.00135 \mathrm{E}-15$ & $2.14468 \mathrm{E}-15$ \\
\hline$E r-165$ & $0.00000 \mathrm{E}+00$ & $0.00000 \mathrm{E}+00$ & $0.00000 \mathrm{E}+00$ & $6.77607 \mathrm{E}-11$ & 7.47727E-11 & $7.88176 \mathrm{E}-11$ \\
\hline$E r-167 m$ & $0.00000 \mathrm{E}+00$ & $0.00000 \mathrm{E}+00$ & $0.00000 \mathrm{E}+00$ & $1.40497 \mathrm{E}-11$ & $1.40333 \mathrm{E}-11$ & $1.38520 \mathrm{E}-11$ \\
\hline Er-169 & $0.00000 \mathrm{E}+00$ & $0.00000 \mathrm{E}+00$ & $0.00000 \mathrm{E}+00$ & $9.86298 \mathrm{E}-06$ & $9.79551 \mathrm{E}-06$ & 9.88881E-06 \\
\hline$E r-171$ & $0.00000 \mathrm{E}+00$ & $0.00000 \mathrm{E}+00$ & $0.00000 \mathrm{E}+00$ & $3.38800 \mathrm{E}-08$ & $3.41296 \mathrm{E}-08$ & 3.49217E-08 \\
\hline Er-172 & $0.00000 \mathrm{E}+00$ & $0.00000 \mathrm{E}+00$ & $0.00000 \mathrm{E}+00$ & $1.08551 \mathrm{E}-07$ & $1.16907 \mathrm{E}-07$ & $18180 \mathrm{E}-07$ \\
\hline Tm-165 & $0.00000 \mathrm{E}+00$ & $0.00000 \mathrm{E}+00$ & $0.00000 \mathrm{E}+00$ & $644 \mathrm{E}-15$ & $1.30541 \mathrm{E}-15$ & $39361 \mathrm{E}-15$ \\
\hline Tm-166 & $0.00000 \mathrm{E}+00$ & $0.00000 \mathrm{E}+00$ & $0.00000 \mathrm{E}+00$ & $4.15875 \mathrm{E}-15$ & $4.34368 \mathrm{E}-15$ & $62915 \mathrm{E}-15$ \\
\hline Tm-167 & $0.00000 \mathrm{E}+00$ & $0.00000 \mathrm{E}+00$ & $0.00000 \mathrm{E}+00$ & $1.54202 \mathrm{E}-12$ & $1.67286 \mathrm{E}-12$ & $.76977 \mathrm{E}-12$ \\
\hline Tm-168 & $0.00000 \mathrm{E}+00$ & $0.00000 \mathrm{E}+00$ & $0.00000 \mathrm{E}+00$ & $1.29795 \mathrm{E}-10$ & $1.36990 \mathrm{E}-10$ & $.44651 \mathrm{E}-10$ \\
\hline$T m-169$ & $0.00000 \mathrm{E}+00$ & $0.00000 \mathrm{E}+00$ & $0.00000 \mathrm{E}+00$ & $3.51670 \mathrm{E}-04$ & $3.47470 \mathrm{E}-04$ & $3.71485 \mathrm{E}-04$ \\
\hline Tm-170 & $0.00000 \mathrm{E}+00$ & $0.00000 \mathrm{E}+00$ & $0.00000 \mathrm{E}+00$ & $4.90015 \mathrm{E}-09$ & $5.21178 \mathrm{E}-09$ & $.54657 \mathrm{E}-09$ \\
\hline Tm-171 & $0.00000 \mathrm{E}+00$ & $0.00000 \mathrm{E}+00$ & $0.00000 \mathrm{E}+00$ & $2.78775 \mathrm{E}-05$ & $2.83536 \mathrm{E}-05$ & $3.00735 \mathrm{E}-05$ \\
\hline Tm-172 & $0.00000 \mathrm{E}+00$ & $0.00000 \mathrm{E}+00$ & $0.00000 \mathrm{E}+00$ & $1.40952 \mathrm{E}-07$ & $1.51790 \mathrm{E}-07$ & $1.53497 \mathrm{E}-07$ \\
\hline$Y b-166$ & $0.00000 \mathrm{E}+00$ & $0.00000 \mathrm{E}+00$ & $0.00000 \mathrm{E}+00$ & $0.00000 \mathrm{E}+00$ & $0.00000 \mathrm{E}+00$ & $0.00000 \mathrm{E}+00$ \\
\hline$Y b-167$ & $0.00000 \mathrm{E}+00$ & $0.00000 \mathrm{E}+00$ & $0.00000 \mathrm{E}+00$ & $1.33842 \mathrm{E}-19$ & $1.32176 \mathrm{E}-19$ & $1.41583 \mathrm{E}-19$ \\
\hline$Y b-168$ & $0.00000 \mathrm{E}+00$ & $0.00000 \mathrm{E}+00$ & $0.00000 \mathrm{E}+00$ & $1.29475 \mathrm{E}-13$ & $1.43802 \mathrm{E}-13$ & $1.59324 \mathrm{E}-13$ \\
\hline$Y b-169$ & $0.00000 \mathrm{E}+00$ & $0.00000 \mathrm{E}+00$ & $0.00000 \mathrm{E}+00$ & $2.63532 \mathrm{E}-13$ & $2.98827 \mathrm{E}-13$ & $3.17295 \mathrm{E}-13$ \\
\hline$Y b-169 m$ & $0.00000 \mathrm{E}+00$ & $0.00000 \mathrm{E}+00$ & $0.00000 \mathrm{E}+00$ & $6.37335 \mathrm{E}-19$ & $7.21403 \mathrm{E}-19$ & 7.60912E-19 \\
\hline$Y b-170$ & $0.00000 \mathrm{E}+00$ & $0.00000 \mathrm{E}+00$ & $0.00000 \mathrm{E}+00$ & 8.38423E-09 & 8.81178E-09 & $9.83226 \mathrm{E}-09$ \\
\hline$Y b-171$ & $0.00000 \mathrm{E}+00$ & $0.00000 \mathrm{E}+00$ & $0.00000 \mathrm{E}+00$ & $1.05686 \mathrm{E}-05$ & $1.08698 \mathrm{E}-05$ & $1.20339 \mathrm{E}-05$ \\
\hline$Y b-172$ & $0.00000 \mathrm{E}+00$ & $0.00000 \mathrm{E}+00$ & $0.00000 \mathrm{E}+00$ & $2.22661 \mathrm{E}-05$ & $2.46808 \mathrm{E}-05$ & $2.65359 \mathrm{E}-05$ \\
\hline Lu-169 & $0.00000 \mathrm{E}+00$ & $0.00000 \mathrm{E}+00$ & $0.00000 \mathrm{E}+00$ & $0.00000 \mathrm{E}+00$ & $0.00000 \mathrm{E}+00$ & $0.00000 \mathrm{E}+00$ \\
\hline$L u-169 m$ & $0.00000 \mathrm{E}+00$ & $0.00000 \mathrm{E}+00$ & $0.00000 \mathrm{E}+00$ & $0.00000 \mathrm{E}+00$ & $0.00000 \mathrm{E}+00$ & $0.00000 \mathrm{E}+00$ \\
\hline$L u-171$ & $0.00000 \mathrm{E}+00$ & $0.00000 \mathrm{E}+00$ & $0.00000 \mathrm{E}+00$ & $3.12851 \mathrm{E}-15$ & $3.60940 \mathrm{E}-15$ & $3.80560 \mathrm{E}-15$ \\
\hline$L u-171 m$ & $0.00000 \mathrm{E}+00$ & $0.00000 \mathrm{E}+00$ & $0.00000 \mathrm{E}+00$ & $1.78226 \mathrm{E}-19$ & $2.05481 \mathrm{E}-19$ & $2.16281 \mathrm{E}-19$ \\
\hline Lu-172 & $0.00000 \mathrm{E}+00$ & $0.00000 \mathrm{E}+00$ & $0.00000 \mathrm{E}+00$ & $3.56809 \mathrm{E}-14$ & $4.11655 \mathrm{E}-14$ & 4.33924E-14 \\
\hline$L u-172 m$ & $0.00000 \mathrm{E}+00$ & $0.00000 \mathrm{E}+00$ & $0.00000 \mathrm{E}+00$ & 6.99354E-18 & $8.06333 \mathrm{E}-18$ & $8.48778 \mathrm{E}-18$ \\
\hline$H f-171$ & $0.00000 \mathrm{E}+00$ & $0.00000 \mathrm{E}+00$ & $0.00000 \mathrm{E}+00$ & $0.00000 \mathrm{E}+00$ & $0.00000 \mathrm{E}+00$ & $0.00000 \mathrm{E}+00$ \\
\hline$H f-172$ & $0.00000 \mathrm{E}+00$ & $0.00000 \mathrm{E}+00$ & $0.00000 \mathrm{E}+00$ & $0.00000 \mathrm{E}+00$ & $0.00000 \mathrm{E}+00$ & $0.00000 \mathrm{E}+00$ \\
\hline $\mathrm{Hg}-206$ & $0.00000 \mathrm{E}+00$ & $0.00000 \mathrm{E}+00$ & $0.00000 \mathrm{E}+00$ & $5.33836 \mathrm{E}-21$ & $7.39882 \mathrm{E}-21$ & $8.37225 \mathrm{E}-21$ \\
\hline$T l-206$ & $0.00000 \mathrm{E}+00$ & $0.00000 \mathrm{E}+00$ & $0.00000 \mathrm{E}+00$ & $3.29695 \mathrm{E}-19$ & $4.46626 \mathrm{E}-19$ & $4.95461 \mathrm{E}-19$ \\
\hline
\end{tabular}




\begin{tabular}{|c|c|c|c|c|c|c|}
\hline \multirow[b]{2}{*}{ Nuclide } & \multicolumn{3}{|c|}{ Fresh Fuel } & \multicolumn{3}{|c|}{ Discharge } \\
\hline & Unit Cell & $\begin{array}{l}\text { Full Core } \\
\text { (NO LBP) }\end{array}$ & Full Core (3) & Unit Cell & $\begin{array}{l}\text { Full Core } \\
\text { (NO LBP) }\end{array}$ & Full Core (3) \\
\hline$T l-207$ & $0.00000 \mathrm{E}+00$ & $0.00000 \mathrm{E}+00$ & $0.00000 \mathrm{E}+00$ & $6.59546 \mathrm{E}-12$ & $8.16195 \mathrm{E}-12$ & $9.17674 \mathrm{E}-12$ \\
\hline$T l-208$ & $0.00000 \mathrm{E}+00$ & $0.00000 \mathrm{E}+00$ & $0.00000 \mathrm{E}+00$ & $1.29809 \mathrm{E}-08$ & $1.60479 \mathrm{E}-08$ & $1.80945 \mathrm{E}-08$ \\
\hline$T l-209$ & $0.00000 \mathrm{E}+00$ & $0.00000 \mathrm{E}+00$ & $0.00000 \mathrm{E}+00$ & 4.74513E-13 & $5.21224 \mathrm{E}-13$ & $5.65316 \mathrm{E}-13$ \\
\hline$T l-210$ & $0.00000 \mathrm{E}+00$ & $0.00000 \mathrm{E}+00$ & $0.00000 \mathrm{E}+00$ & $9.55035 \mathrm{E}-20$ & $1.02797 \mathrm{E}-19$ & $1.18277 \mathrm{E}-19$ \\
\hline $\mathrm{Pb}-206$ & $0.00000 \mathrm{E}+00$ & $0.00000 \mathrm{E}+00$ & $0.00000 \mathrm{E}+00$ & 5.19027E-09 & 7.40113E-09 & $9.34170 \mathrm{E}-09$ \\
\hline$P b-207$ & $0.00000 \mathrm{E}+00$ & $0.00000 \mathrm{E}+00$ & $0.00000 \mathrm{E}+00$ & $7.02100 \mathrm{E}-07$ & $8.74929 \mathrm{E}-07$ & $1.03170 \mathrm{E}-06$ \\
\hline$P b-208$ & $0.00000 \mathrm{E}+00$ & $0.00000 \mathrm{E}+00$ & $0.00000 \mathrm{E}+00$ & $3.20074 \mathrm{E}-03$ & $3.95060 \mathrm{E}-03$ & $4.61029 \mathrm{E}-03$ \\
\hline$P b-209$ & $0.00000 \mathrm{E}+00$ & $0.00000 \mathrm{E}+00$ & $0.00000 \mathrm{E}+00$ & 2.01652E-09 & $2.21599 \mathrm{E}-09$ & $2.40365 \mathrm{E}-09$ \\
\hline$P b-210$ & $0.00000 \mathrm{E}+00$ & $0.00000 \mathrm{E}+00$ & $0.00000 \mathrm{E}+00$ & 4.10371E-07 & $5.68767 \mathrm{E}-07$ & $6.43596 \mathrm{E}-07$ \\
\hline$P b-211$ & $0.00000 \mathrm{E}+00$ & $0.00000 \mathrm{E}+00$ & $0.00000 \mathrm{E}+00$ & 0238E-11 & $6.31422 \mathrm{E}-11$ & $7.09926 \mathrm{E}-11$ \\
\hline$P b-212$ & $0.00000 \mathrm{E}+00$ & $0.00000 \mathrm{E}+00$ & $0.00000 \mathrm{E}+00$ & $9940 \mathrm{E}-06$ & $9.51848 \mathrm{E}-06$ & $3 \mathrm{E}-05$ \\
\hline$P b-214$ & $0.00000 \mathrm{E}+00$ & $0.00000 \mathrm{E}+00$ & $0.00000 \mathrm{E}+00$ & $9.55308 \mathrm{E}-15$ & $1.02827 \mathrm{E}-14$ & $1.18311 \mathrm{E}-14$ \\
\hline$B i-209$ & $0.00000 \mathrm{E}+00$ & $0.00000 \mathrm{E}+00$ & $0.00000 \mathrm{E}+00$ & $3.35685 \mathrm{E}-06$ & $3.59097 \mathrm{E}-06$ & $3.97459 \mathrm{E}-06$ \\
\hline$B i-210$ & $0.00000 \mathrm{E}+00$ & $0.00000 \mathrm{E}+00$ & $0.00000 \mathrm{E}+00$ & $2.54738 \mathrm{E}-10$ & $3.51874 \mathrm{E}-10$ & $3.98359 \mathrm{E}-10$ \\
\hline$B i-211$ & $0.00000 \mathrm{E}+00$ & $0.00000 \mathrm{E}+00$ & $0.00000 \mathrm{E}+00$ & $3.02464 \mathrm{E}-12$ & $3.74302 \mathrm{E}-12$ & $4.20840 \mathrm{E}-12$ \\
\hline$B i-212$ & $0.00000 \mathrm{E}+00$ & $0.00000 \mathrm{E}+00$ & $0.00000 \mathrm{E}+00$ & 7.30148E-07 & $9.02654 \mathrm{E}-07$ & $1.01778 \mathrm{E}-06$ \\
\hline$B i-213$ & $0.00000 \mathrm{E}+00$ & $0.00000 \mathrm{E}+00$ & $0.00000 \mathrm{E}+00$ & $4.79518 \mathrm{E}-10$ & $5.26717 \mathrm{E}-10$ & $5.71274 \mathrm{E}-10$ \\
\hline$B i-214$ & $0.00000 \mathrm{E}+00$ & $0.00000 \mathrm{E}+00$ & $0.00000 \mathrm{E}+00$ & $7.09453 \mathrm{E}-15$ & $7.63635 \mathrm{E}-15$ & $8.78631 \mathrm{E}-15$ \\
\hline Po-210 & $0.00000 \mathrm{E}+00$ & $0.00000 \mathrm{E}+00$ & $0.00000 \mathrm{E}+00$ & $4.37509 \mathrm{E}-09$ & $6.11486 \mathrm{E}-09$ & $7.18599 \mathrm{E}-09$ \\
\hline Po-211 & $0.00000 \mathrm{E}+00$ & $0.00000 \mathrm{E}+00$ & $0.00000 \mathrm{E}+00$ & $3.35480 \mathrm{E}-17$ & $4.15159 \mathrm{E}-17$ & 4.66777E-17 \\
\hline Po-212 & $0.00000 \mathrm{E}+00$ & $0.00000 \mathrm{E}+00$ & $0.00000 \mathrm{E}+00$ & $3.84944 \mathrm{E}-17$ & 4.75894E-17 & $5.36585 \mathrm{E}-17$ \\
\hline Po-213 & $0.00000 \mathrm{E}+00$ & $0.00000 \mathrm{E}+00$ & $0.00000 \mathrm{E}+00$ & $7.20920 \mathrm{E}-19$ & 7.91887E-19 & $8869 \mathrm{E}-19$ \\
\hline Po-214 & $0.00000 \mathrm{E}+00$ & $0.00000 \mathrm{E}+00$ & $0.00000 \mathrm{E}+00$ & $3.02451 \mathrm{E}-18$ & 4.06736E-18 & $4.19500 \mathrm{E}-18$ \\
\hline Po-215 & $0.00000 \mathrm{E}+00$ & $0.00000 \mathrm{E}+00$ & $0.00000 \mathrm{E}+00$ & $4.27521 \mathrm{E}-17$ & $5.29060 \mathrm{E}-17$ & $4834 \mathrm{E}-17$ \\
\hline Po-216 & $0.00000 \mathrm{E}+00$ & $0.00000 \mathrm{E}+00$ & $0.00000 \mathrm{E}+00$ & $2.97447 \mathrm{E}-11$ & $3.67726 \mathrm{E}-11$ & $4.14580 \mathrm{E}-11$ \\
\hline Po-218 & $0.00000 \mathrm{E}+00$ & $0.00000 \mathrm{E}+00$ & $0.00000 \mathrm{E}+00$ & $1.12604 \mathrm{E}-15$ & $1.21203 \mathrm{E}-15$ & $1.39454 \mathrm{E}-15$ \\
\hline$A t-217$ & $0.00000 \mathrm{E}+00$ & $0.00000 \mathrm{E}+00$ & $0.00000 \mathrm{E}+00$ & $5.76969 \mathrm{E}-15$ & $6.33765 \mathrm{E}-15$ & $6.87373 \mathrm{E}-15$ \\
\hline At-218 & $0.00000 \mathrm{E}+00$ & $0.00000 \mathrm{E}+00$ & $0.00000 \mathrm{E}+00$ & $1.81619 \mathrm{E}-21$ & $1.95490 \mathrm{E}-21$ & $2.24926 \mathrm{E}-21$ \\
\hline$R n-217$ & $0.00000 \mathrm{E}+00$ & $0.00000 \mathrm{E}+00$ & $0.00000 \mathrm{E}+00$ & $6.75213 \mathrm{E}-21$ & $7.41685 \mathrm{E}-21$ & $8.04418 \mathrm{E}-21$ \\
\hline$R n-218$ & $0.00000 \mathrm{E}+00$ & $0.00000 \mathrm{E}+00$ & $0.00000 \mathrm{E}+00$ & $6.56158 \mathrm{E}-16$ & $8.82459 \mathrm{E}-16$ & $9.10125 \mathrm{E}-16$ \\
\hline$R n-219$ & $0.00000 \mathrm{E}+00$ & $0.00000 \mathrm{E}+00$ & $0.00000 \mathrm{E}+00$ & 9.68307E-14 & $1.19829 \mathrm{E}-13$ & $1.34727 \mathrm{E}-13$ \\
\hline$R n-220$ & $0.00000 \mathrm{E}+00$ & $0.00000 \mathrm{E}+00$ & $0.00000 \mathrm{E}+00$ & $1.16172 \mathrm{E}-08$ & $1.43621 \mathrm{E}-08$ & $1.61921 \mathrm{E}-08$ \\
\hline$R n-222$ & $0.00000 \mathrm{E}+00$ & $0.00000 \mathrm{E}+00$ & $0.00000 \mathrm{E}+00$ & $2.03672 \mathrm{E}-12$ & $2.19227 \mathrm{E}-12$ & $2.52238 \mathrm{E}-12$ \\
\hline$F r-221$ & $0.00000 \mathrm{E}+00$ & $0.00000 \mathrm{E}+00$ & $0.00000 \mathrm{E}+00$ & $5.34868 \mathrm{E}-11$ & $5.87522 \mathrm{E}-11$ & $6.37219 \mathrm{E}-11$ \\
\hline$F r-222$ & $0.00000 \mathrm{E}+00$ & $0.00000 \mathrm{E}+00$ & $0.00000 \mathrm{E}+00$ & $5.28840 \mathrm{E}-16$ & $5.89856 \mathrm{E}-16$ & $6.17207 \mathrm{E}-16$ \\
\hline$F r-223$ & $0.00000 \mathrm{E}+00$ & $0.00000 \mathrm{E}+00$ & $0.00000 \mathrm{E}+00$ & $5.11119 \mathrm{E}-13$ & $6.26099 \mathrm{E}-13$ & $6.96320 \mathrm{E}-13$ \\
\hline
\end{tabular}




\section{Depletion Analysis of Modular High Temperature Gas-cooled Reactor Loaded with LEU/Thorium Fuel}

February 2012

\begin{tabular}{|c|c|c|c|c|c|c|}
\hline \multirow[b]{2}{*}{ Nuclide } & \multicolumn{3}{|c|}{ Fresh Fuel } & \multicolumn{3}{|c|}{ Discharge } \\
\hline & Unit Cell & $\begin{array}{l}\text { Full Core } \\
(N O L B P)\end{array}$ & Full Core (3) & Unit Cell & $\begin{array}{l}\text { Full Core } \\
\text { (NO LBP) }\end{array}$ & Full Core (3) \\
\hline$R a-222$ & $0.00000 \mathrm{E}+00$ & $0.00000 \mathrm{E}+00$ & $0.00000 \mathrm{E}+00$ & $7.25505 \mathrm{E}-13$ & $9.75723 \mathrm{E}-13$ & $1.00631 \mathrm{E}-12$ \\
\hline$R a-223$ & $0.00000 \mathrm{E}+00$ & $0.00000 \mathrm{E}+00$ & $0.00000 \mathrm{E}+00$ & $2.45899 \mathrm{E}-08$ & $3.04302 \mathrm{E}-08$ & $3.42135 \mathrm{E}-08$ \\
\hline$R a-224$ & $0.00000 \mathrm{E}+00$ & $0.00000 \mathrm{E}+00$ & $0.00000 \mathrm{E}+00$ & $6.72771 \mathrm{E}-05$ & $8.31730 \mathrm{E}-05$ & 9.37704E-05 \\
\hline$R a-225$ & $0.00000 \mathrm{E}+00$ & $0.00000 \mathrm{E}+00$ & $0.00000 \mathrm{E}+00$ & $2.58048 \mathrm{E}-07$ & $2.84008 \mathrm{E}-07$ & 3.07097E-07 \\
\hline$R a-226$ & $0.00000 \mathrm{E}+00$ & $0.00000 \mathrm{E}+00$ & $0.00000 \mathrm{E}+00$ & $3.21905 \mathrm{E}-07$ & $3.46481 \mathrm{E}-07$ & $3.98216 \mathrm{E}-07$ \\
\hline$R a-227$ & $0.00000 \mathrm{E}+00$ & $0.00000 \mathrm{E}+00$ & $0.00000 \mathrm{E}+00$ & $6.01293 \mathrm{E}-12$ & $6.37869 \mathrm{E}-12$ & $7.09083 \mathrm{E}-12$ \\
\hline$R a-228$ & $0.00000 \mathrm{E}+00$ & $0.00000 \mathrm{E}+00$ & $0.00000 \mathrm{E}+00$ & $4.35845 \mathrm{E}-05$ & $4.36038 \mathrm{E}-05$ & $4.35462 \mathrm{E}-05$ \\
\hline$A c-225$ & $0.00000 \mathrm{E}+00$ & $0.00000 \mathrm{E}+00$ & $0.00000 \mathrm{E}+00$ & $1.60039 \mathrm{E}-07$ & $1.75793 \mathrm{E}-07$ & $1.90662 \mathrm{E}-07$ \\
\hline$A c-226$ & $0.00000 \mathrm{E}+00$ & $0.00000 \mathrm{E}+00$ & $0.00000 \mathrm{E}+00$ & $1.11358 \mathrm{E}-09$ & $1.24207 \mathrm{E}-09$ & 6E-09 \\
\hline$A c-227$ & $0.00000 \mathrm{E}+00$ & $0.00000 \mathrm{E}+00$ & $0.00000 \mathrm{E}+00$ & $1.96249 \mathrm{E}-05$ & $2.40397 \mathrm{E}-05$ & $2.67358 \mathrm{E}-05$ \\
\hline Ac- 228 & $0.00000 \mathrm{E}+00$ & $0.00000 \mathrm{E}+00$ & $0.00000 \mathrm{E}+00$ & 4.45390E- 08 & 5.41937E-08 & 77284E-08 \\
\hline Th-226 & $0.00000 \mathrm{E}+00$ & $0.00000 \mathrm{E}+00$ & $0.00000 \mathrm{E}+00$ & $6498 \mathrm{E}-11$ & $4.79451 \mathrm{E}-11$ & $94482 \mathrm{E}-11$ \\
\hline Th-227 & $0.00000 \mathrm{E}+00$ & $0.00000 \mathrm{E}+00$ & $0.00000 \mathrm{E}+00$ & $4.07726 \mathrm{E}-08$ & $5.04470 \mathrm{E}-08$ & $5 \mathrm{E}-08$ \\
\hline Th-228 & $0.00000 \mathrm{E}+00$ & $0.00000 \mathrm{E}+00$ & $0.00000 \mathrm{E}+00$ & $1.32394 \mathrm{E}-02$ & $1.63684 \mathrm{E}-02$ & $95 \mathrm{E}-02$ \\
\hline Th-229 & $0.00000 \mathrm{E}+00$ & $0.00000 \mathrm{E}+00$ & $0.00000 \mathrm{E}+00$ & $3.52220 \mathrm{E}-02$ & 3.62954E-02 & $3.90088 \mathrm{E}-02$ \\
\hline Th-230 & $0.00000 \mathrm{E}+00$ & $0.00000 \mathrm{E}+00$ & $0.00000 \mathrm{E}+00$ & $3.16941 \mathrm{E}-02$ & 3.35546E-02 & 3.63819E-02 \\
\hline Th-231 & $0.00000 \mathrm{E}+00$ & $0.00000 \mathrm{E}+00$ & $0.00000 \mathrm{E}+00$ & $8.98217 \mathrm{E}-03$ & $1.11422 \mathrm{E}-02$ & $1.08415 \mathrm{E}-02$ \\
\hline Th-235 & $0.00000 \mathrm{E}+00$ & $0.00000 \mathrm{E}+00$ & $0.00000 \mathrm{E}+00$ & $3.51668 \mathrm{E}-08$ & $3.83040 \mathrm{E}-08$ & 3.43204E-08 \\
\hline $\mathrm{Pa}-229$ & $0.00000 \mathrm{E}+00$ & $0.00000 \mathrm{E}+00$ & $0.00000 \mathrm{E}+00$ & $1.49029 \mathrm{E}-11$ & $1.98025 \mathrm{E}-11$ & 1.99184E-11 \\
\hline $\mathrm{Pa}-230$ & $0.00000 \mathrm{E}+00$ & $0.00000 \mathrm{E}+00$ & $0.00000 \mathrm{E}+00$ & $1.97654 \mathrm{E}-07$ & $3.02808 \mathrm{E}-07$ & 3.08134E-07 \\
\hline $\mathrm{Pa}-234$ & $0.00000 \mathrm{E}+00$ & $0.00000 \mathrm{E}+00$ & $0.00000 \mathrm{E}+00$ & $1.97288 \mathrm{E}-01$ & $1.99855 \mathrm{E}-01$ & $1.86386 \mathrm{E}-01$ \\
\hline $\mathrm{Pa}-235$ & $0.00000 \mathrm{E}+00$ & $0.00000 \mathrm{E}+00$ & $0.00000 \mathrm{E}+00$ & $1.19370 \mathrm{E}-07$ & $1.30019 \mathrm{E}-07$ & $1.16497 \mathrm{E}-07$ \\
\hline$U-230$ & $0.00000 \mathrm{E}+00$ & $0.00000 \mathrm{E}+00$ & $0.00000 \mathrm{E}+00$ & $1.95484 \mathrm{E}-08$ & $2.99606 \mathrm{E}-08$ & $3.06315 \mathrm{E}-08$ \\
\hline$U-231$ & $0.00000 \mathrm{E}+00$ & $0.00000 \mathrm{E}+00$ & $0.00000 \mathrm{E}+00$ & $1.50604 \mathrm{E}-09$ & 2.15554E-09 & $2.26707 \mathrm{E}-09$ \\
\hline$U-242$ & $0.00000 \mathrm{E}+00$ & $0.00000 \mathrm{E}+00$ & $0.00000 \mathrm{E}+00$ & $1.48370 \mathrm{E}-14$ & $1.49281 \mathrm{E}-14$ & $1.29861 \mathrm{E}-14$ \\
\hline$N p-233$ & $0.00000 \mathrm{E}+00$ & $0.00000 \mathrm{E}+00$ & $0.00000 \mathrm{E}+00$ & $2.01569 \mathrm{E}-22$ & $2.76043 \mathrm{E}-22$ & $.94284 \mathrm{E}-22$ \\
\hline$N p-234$ & $0.00000 \mathrm{E}+00$ & $0.00000 \mathrm{E}+00$ & $0.00000 \mathrm{E}+00$ & $1.13313 \mathrm{E}-14$ & $1.56108 \mathrm{E}-14$ & $1.63589 \mathrm{E}-14$ \\
\hline$N p-240$ & $0.00000 \mathrm{E}+00$ & $0.00000 \mathrm{E}+00$ & $0.00000 \mathrm{E}+00$ & $3.72492 \mathrm{E}-03$ & $3.73539 \mathrm{E}-03$ & $3.48206 \mathrm{E}-03$ \\
\hline$N p-241$ & $0.00000 \mathrm{E}+00$ & $0.00000 \mathrm{E}+00$ & $0.00000 \mathrm{E}+00$ & $1.66542 \mathrm{E}-09$ & $1.62962 \mathrm{E}-09$ & $1.47116 \mathrm{E}-09$ \\
\hline$N p-242$ & $0.00000 \mathrm{E}+00$ & $0.00000 \mathrm{E}+00$ & $0.00000 \mathrm{E}+00$ & $1.94293 \mathrm{E}-15$ & $1.95486 \mathrm{E}-15$ & $1.70056 \mathrm{E}-15$ \\
\hline$P u-234$ & $0.00000 \mathrm{E}+00$ & $0.00000 \mathrm{E}+00$ & $0.00000 \mathrm{E}+00$ & $2.93754 \mathrm{E}-20$ & $3.96901 \mathrm{E}-20$ & $4.35490 \mathrm{E}-20$ \\
\hline$P u-235$ & $0.00000 \mathrm{E}+00$ & $0.00000 \mathrm{E}+00$ & $0.00000 \mathrm{E}+00$ & $4.21650 \mathrm{E}-17$ & $5.80912 \mathrm{E}-17$ & 6.29804 \\
\hline$P u-237 m$ & $0.00000 \mathrm{E}+00$ & $0.00000 \mathrm{E}+00$ & $0.00000 \mathrm{E}+00$ & $5.79174 \mathrm{E}-17$ & $6.58878 \mathrm{E}-17$ & $7.38855 \mathrm{E}-17$ \\
\hline$P u-245$ & $0.00000 \mathrm{E}+00$ & $0.00000 \mathrm{E}+00$ & $0.00000 \mathrm{E}+00$ & $1.65561 \mathrm{E}-06$ & $1.52852 \mathrm{E}-06$ & $9.90570 \mathrm{E}-07$ \\
\hline$A m-238$ & $0.00000 \mathrm{E}+00$ & $0.00000 \mathrm{E}+00$ & $0.00000 \mathrm{E}+00$ & $1.68286 \mathrm{E}-26$ & $2.28985 \mathrm{E}-26$ & $2.55039 \mathrm{E}-26$ \\
\hline Am-239 & $0.00000 \mathrm{E}+00$ & $0.00000 \mathrm{E}+00$ & $0.00000 \mathrm{E}+00$ & $5.40575 \mathrm{E}-11$ & 5.93834E-11 & $6.61396 \mathrm{E}-11$ \\
\hline
\end{tabular}




\begin{tabular}{|c|c|c|c|c|c|c|}
\hline \multirow[b]{2}{*}{ Nuclide } & \multicolumn{3}{|c|}{ Fresh Fuel } & \multicolumn{3}{|c|}{ Discharge } \\
\hline & Unit Cell & $\begin{array}{l}\text { Full Core } \\
\text { (NO LBP) }\end{array}$ & Full Core (3) & Unit Cell & $\begin{array}{l}\text { Full Core } \\
\text { (NO LBP) }\end{array}$ & Full Core (3) \\
\hline Am-240 & $0.00000 \mathrm{E}+00$ & $0.00000 \mathrm{E}+00$ & $0.00000 \mathrm{E}+00$ & $1.18496 \mathrm{E}-07$ & $1.35222 \mathrm{E}-07$ & $1.50954 \mathrm{E}-07$ \\
\hline$A m-245$ & $0.00000 \mathrm{E}+00$ & $0.00000 \mathrm{E}+00$ & $0.00000 \mathrm{E}+00$ & $3.80447 \mathrm{E}-06$ & $3.47398 \mathrm{E}-06$ & 3.52333E-05 \\
\hline $\mathrm{Cm}-238$ & $0.00000 \mathrm{E}+00$ & $0.00000 \mathrm{E}+00$ & $0.00000 \mathrm{E}+00$ & $2.74823 \mathrm{E}-26$ & $3.73943 \mathrm{E}-26$ & 4.16491E-26 \\
\hline Cm-239 & $0.00000 \mathrm{E}+00$ & $0.00000 \mathrm{E}+00$ & $0.00000 \mathrm{E}+00$ & $6.77000 \mathrm{E}-21$ & $9.03954 \mathrm{E}-21$ & $1.00845 \mathrm{E}-20$ \\
\hline $\mathrm{Cm}-246$ & $0.00000 \mathrm{E}+00$ & $0.00000 \mathrm{E}+00$ & $0.00000 \mathrm{E}+00$ & $2.16884 \mathrm{E}-10$ & $1.84332 \mathrm{E}-10$ & $2.25675 \mathrm{E}-10$ \\
\hline$L i-7$ & $0.00000 \mathrm{E}+00$ & $0.00000 \mathrm{E}+00$ & $0.00000 \mathrm{E}+00$ & $2.91193 \mathrm{E}-24$ & $4.25238 \mathrm{E}-24$ & $4.19039 \mathrm{E}-24$ \\
\hline$B e-7$ & $0.00000 \mathrm{E}+00$ & $0.00000 \mathrm{E}+00$ & $0.00000 \mathrm{E}+00$ & $0.00000 \mathrm{E}+00$ & $0.00000 \mathrm{E}+00$ & $0.00000 \mathrm{E}+00$ \\
\hline$B e-9$ & $0.00000 \mathrm{E}+00$ & $0.00000 \mathrm{E}+00$ & $0.00000 \mathrm{E}+00$ & $8.37547 \mathrm{E}-19$ & $1.51643 \mathrm{E}-18$ & $97741 \mathrm{E}-18$ \\
\hline$B-10$ & $0.00000 \mathrm{E}+00$ & $0.00000 \mathrm{E}+00$ & $0.00000 \mathrm{E}+00$ & $2.33451 \mathrm{E}-24$ & $3.35235 \mathrm{E}-24$ & $19942 \mathrm{E}-24$ \\
\hline$B-11$ & $0.00000 \mathrm{E}+00$ & $0.00000 \mathrm{E}+00$ & $0.00000 \mathrm{E}+00$ & $2.47517 \mathrm{E}-14$ & $3.24482 \mathrm{E}-14$ & $.11291 \mathrm{E}-14$ \\
\hline$C-12$ & $0.00000 \mathrm{E}+00$ & $0.00000 \mathrm{E}+00$ & $0.00000 \mathrm{E}+00$ & $4.05818 \mathrm{E}-12$ & $5.90114 \mathrm{E}-12$ & $101 \mathrm{E}-12$ \\
\hline$O-17$ & $0.00000 \mathrm{E}+00$ & $0.00000 \mathrm{E}+00$ & $0.00000 \mathrm{E}+00$ & $5.54078 \mathrm{E}-02$ & $5.60680 \mathrm{E}-02$ & $76 \mathrm{E}-02$ \\
\hline Co- $158 m$ & $0.00000 \mathrm{E}+00$ & $0.00000 \mathrm{E}+00$ & $0.00000 \mathrm{E}+00$ & $0.00000 \mathrm{E}+00$ & $0.00000 \mathrm{E}+00$ & $.00000 \mathrm{E}+00$ \\
\hline Ga-69 & $0.00000 \mathrm{E}+00$ & $0.00000 \mathrm{E}+00$ & $0.00000 \mathrm{E}+00$ & $8.92380 \mathrm{E}-04$ & $8.97126 \mathrm{E}-04$ & $9.19248 \mathrm{E}-04$ \\
\hline Ga-71 & $0.00000 \mathrm{E}+00$ & $0.00000 \mathrm{E}+00$ & $0.00000 \mathrm{E}+00$ & $7.78758 \mathrm{E}-03$ & $7.79839 \mathrm{E}-03$ & $8.02103 \mathrm{E}-03$ \\
\hline$G e-70$ & $0.00000 \mathrm{E}+00$ & $0.00000 \mathrm{E}+00$ & $0.00000 \mathrm{E}+00$ & $6.38214 \mathrm{E}-06$ & $6.62910 \mathrm{E}-06$ & $6.80385 \mathrm{E}-06$ \\
\hline Ge-72 & $0.00000 \mathrm{E}+00$ & $0.00000 \mathrm{E}+00$ & $0.00000 \mathrm{E}+00$ & $2.15314 \mathrm{E}-02$ & $2.15945 \mathrm{E}-02$ & $2.21413 \mathrm{E}-02$ \\
\hline Ge-73 & $0.00000 \mathrm{E}+00$ & $0.00000 \mathrm{E}+00$ & $0.00000 \mathrm{E}+00$ & $6.17123 \mathrm{E}-02$ & $6.19604 \mathrm{E}-02$ & $6.31289 \mathrm{E}-02$ \\
\hline Ge-74 & $0.00000 \mathrm{E}+00$ & $0.00000 \mathrm{E}+00$ & $0.00000 \mathrm{E}+00$ & $1.76029 \mathrm{E}-01$ & $1.76910 \mathrm{E}-01$ & $1.79291 \mathrm{E}-01$ \\
\hline$G e-76$ & $0.00000 \mathrm{E}+00$ & $0.00000 \mathrm{E}+00$ & $0.00000 \mathrm{E}+00$ & $1.23890 \mathrm{E}+00$ & $1.24780 \mathrm{E}+00$ & $1.25435 \mathrm{E}+00$ \\
\hline As-74 & $0.00000 \mathrm{E}+00$ & $0.00000 \mathrm{E}+00$ & $0.00000 \mathrm{E}+00$ & $8.74201 \mathrm{E}-09$ & $9.39911 \mathrm{E}-09$ & 9.34594E-09 \\
\hline As-75 & $0.00000 \mathrm{E}+00$ & $0.00000 \mathrm{E}+00$ & $0.00000 \mathrm{E}+00$ & $5.01315 \mathrm{E}-01$ & $5.04195 \mathrm{E}-01$ & $5.09385 \mathrm{E}-01$ \\
\hline $\mathrm{Se}-74$ & $0.00000 \mathrm{E}+00$ & $0.00000 \mathrm{E}+00$ & $0.00000 \mathrm{E}+00$ & $5.00855 \mathrm{E}-08$ & $5.21872 \mathrm{E}-08$ & $5.48995 \mathrm{E}-08$ \\
\hline $\mathrm{Se}-76$ & $0.00000 \mathrm{E}+00$ & $0.00000 \mathrm{E}+00$ & $0.00000 \mathrm{E}+00$ & $1.00586 \mathrm{E}-02$ & $1.04258 \mathrm{E}-02$ & $1.05474 \mathrm{E}-02$ \\
\hline $\mathrm{Se}-77$ & $0.00000 \mathrm{E}+00$ & $0.00000 \mathrm{E}+00$ & $0.00000 \mathrm{E}+00$ & $2.80138 \mathrm{E}+00$ & $2.81444 \mathrm{E}+00$ & $2.82654 \mathrm{E}+00$ \\
\hline $\mathrm{Se}-78$ & $0.00000 \mathrm{E}+00$ & $0.00000 \mathrm{E}+00$ & $0.00000 \mathrm{E}+00$ & $7.71110 \mathrm{E}+00$ & $7.74393 \mathrm{E}+00$ & $7.74889 \mathrm{E}+00$ \\
\hline Se-79 & $0.00000 \mathrm{E}+00$ & $0.00000 \mathrm{E}+00$ & $0.00000 \mathrm{E}+00$ & $1.59538 \mathrm{E}+01$ & $1.59943 \mathrm{E}+01$ & $1.60677 \mathrm{E}+01$ \\
\hline Se-80 & $0.00000 \mathrm{E}+00$ & $0.00000 \mathrm{E}+00$ & $0.00000 \mathrm{E}+00$ & $4.43744 \mathrm{E}+01$ & $4.45658 \mathrm{E}+01$ & $4.44063 \mathrm{E}+01$ \\
\hline $\mathrm{Se}-82$ & $0.00000 \mathrm{E}+00$ & $0.00000 \mathrm{E}+00$ & $0.00000 \mathrm{E}+00$ & $1.12899 \mathrm{E}+02$ & $1.13307 \mathrm{E}+02$ & $1.12989 \mathrm{E}+02$ \\
\hline Br-79 & $0.00000 \mathrm{E}+00$ & $0.00000 \mathrm{E}+00$ & $0.00000 \mathrm{E}+00$ & $8.65358 \mathrm{E}-03$ & $8.67681 \mathrm{E}-03$ & $8.70004 \mathrm{E}-03$ \\
\hline$B r-81$ & $0.00000 \mathrm{E}+00$ & $0.00000 \mathrm{E}+00$ & $0.00000 \mathrm{E}+00$ & $7.04447 \mathrm{E}+01$ & $7.05773 \mathrm{E}+01$ & $7.04798 \mathrm{E}+01$ \\
\hline$K r-78$ & $0.00000 \mathrm{E}+00$ & $0.00000 \mathrm{E}+00$ & $0.00000 \mathrm{E}+00$ & $2.01930 \mathrm{E}-11$ & $1.96778 \mathrm{E}-11$ & $2.07365 \mathrm{E}-11$ \\
\hline$K r-80$ & $0.00000 \mathrm{E}+00$ & $0.00000 \mathrm{E}+00$ & $0.00000 \mathrm{E}+00$ & 7.00164E-04 & $7.14376 \mathrm{E}-04$ & $7.24767 \mathrm{E}-04$ \\
\hline$K r-82$ & $0.00000 \mathrm{E}+00$ & $0.00000 \mathrm{E}+00$ & $0.00000 \mathrm{E}+00$ & $1.22426 \mathrm{E}+00$ & $1.27339 \mathrm{E}+00$ & $1.27592 \mathrm{E}+00$ \\
\hline$K r-83$ & $0.00000 \mathrm{E}+00$ & $0.00000 \mathrm{E}+00$ & $0.00000 \mathrm{E}+00$ & $1.42716 \mathrm{E}+02$ & $1.42763 \mathrm{E}+02$ & $1.43481 \mathrm{E}+02$ \\
\hline$K r-84$ & $0.00000 \mathrm{E}+00$ & $0.00000 \mathrm{E}+00$ & $0.00000 \mathrm{E}+00$ & $3.20454 \mathrm{E}+02$ & $3.22602 \mathrm{E}+02$ & $3.20032 \mathrm{E}+02$ \\
\hline
\end{tabular}




\section{Depletion Analysis of Modular High Temperature Gas-cooled Reactor Loaded with LEU/Thorium Fuel}

February 2012

73

\begin{tabular}{|c|c|c|c|c|c|c|}
\hline \multirow[b]{2}{*}{ Nuclide } & \multicolumn{3}{|c|}{ Fresh Fuel } & \multicolumn{3}{|c|}{ Discharge } \\
\hline & Unit Cell & $\begin{array}{l}\text { Full Core } \\
(N O L B P)\end{array}$ & Full Core (3) & Unit Cell & $\begin{array}{l}\text { Full Core } \\
\text { (NO LBP) }\end{array}$ & Full Core (3) \\
\hline$K r-85$ & $0.00000 \mathrm{E}+00$ & $0.00000 \mathrm{E}+00$ & $0.00000 \mathrm{E}+00$ & $7.30160 \mathrm{E}+01$ & $7.34560 \mathrm{E}+01$ & $7.31443 \mathrm{E}+01$ \\
\hline$K r-86$ & $0.00000 \mathrm{E}+00$ & $0.00000 \mathrm{E}+00$ & $0.00000 \mathrm{E}+00$ & $5.80488 \mathrm{E}+02$ & $5.83917 \mathrm{E}+02$ & $5.78641 \mathrm{E}+02$ \\
\hline$R b-85$ & $0.00000 \mathrm{E}+00$ & $0.00000 \mathrm{E}+00$ & $0.00000 \mathrm{E}+00$ & $2.20403 \mathrm{E}+02$ & $2.21600 \mathrm{E}+02$ & $2.20550 \mathrm{E}+02$ \\
\hline$R b-86$ & $0.00000 \mathrm{E}+00$ & $0.00000 \mathrm{E}+00$ & $0.00000 \mathrm{E}+00$ & $4.02271 \mathrm{E}-02$ & 4.35815E-02 & 4.18557E-02 \\
\hline$R b-87$ & $0.00000 \mathrm{E}+00$ & $0.00000 \mathrm{E}+00$ & $0.00000 \mathrm{E}+00$ & $8.62256 \mathrm{E}+02$ & $8.67015 \mathrm{E}+02$ & $8.61177 \mathrm{E}+02$ \\
\hline$S r-84$ & $0.00000 \mathrm{E}+00$ & $0.00000 \mathrm{E}+00$ & $0.00000 \mathrm{E}+00$ & $1.16277 \mathrm{E}-06$ & $1.34504 \mathrm{E}-06$ & $1.41502 \mathrm{E}-06$ \\
\hline$S r-86$ & $0.00000 \mathrm{E}+00$ & $0.00000 \mathrm{E}+00$ & $0.00000 \mathrm{E}+00$ & $6.12632 \mathrm{E}-01$ & 6.55913E-01 & $6.55009 \mathrm{E}-01$ \\
\hline$S r-87$ & $0.00000 \mathrm{E}+00$ & $0.00000 \mathrm{E}+00$ & $0.00000 \mathrm{E}+00$ & 4.03413E-03 & $4.12850 \mathrm{E}-03$ & $4.25220 \mathrm{E}-03$ \\
\hline$S r-88$ & $0.00000 \mathrm{E}+00$ & $0.00000 \mathrm{E}+00$ & $0.00000 \mathrm{E}+00$ & $1.21458 \mathrm{E}+03$ & $1.22143 \mathrm{E}+03$ & $1.21267 \mathrm{E}+03$ \\
\hline$S r-89$ & $0.00000 \mathrm{E}+00$ & $0.00000 \mathrm{E}+00$ & $0.00000 \mathrm{E}+00$ & $1.13017 \mathrm{E}+02$ & $1.13924 \mathrm{E}+02$ & $1.13942 \mathrm{E}+02$ \\
\hline$S r-90$ & $0.00000 \mathrm{E}+00$ & $0.00000 \mathrm{E}+00$ & $0.00000 \mathrm{E}+00$ & $1.93709 \mathrm{E}+03$ & $1.94740 \mathrm{E}+03$ & $1.93107 \mathrm{E}+03$ \\
\hline$Y-89$ & $0.00000 \mathrm{E}+00$ & $0.00000 \mathrm{E}+00$ & $0.00000 \mathrm{E}+00$ & $1.50380 \mathrm{E}+03$ & $1.51170 \mathrm{E}+03$ & $1.49885 \mathrm{E}+03$ \\
\hline$Y-90$ & $0.00000 \mathrm{E}+00$ & $0.00000 \mathrm{E}+00$ & $0.00000 \mathrm{E}+00$ & $5.15275 \mathrm{E}-01$ & 5.18791E-01 & $.13474 \mathrm{E}-01$ \\
\hline$Y-91$ & $0.00000 \mathrm{E}+00$ & $0.00000 \mathrm{E}+00$ & $0.00000 \mathrm{E}+00$ & $1.62726 \mathrm{E}+02$ & $1.63793 \mathrm{E}+02$ & $1.63788 \mathrm{E}+02$ \\
\hline$Z r-90$ & $0.00000 \mathrm{E}+00$ & $0.00000 \mathrm{E}+00$ & $0.00000 \mathrm{E}+00$ & $6.64940 \mathrm{E}+01$ & $6.68181 \mathrm{E}+01$ & $6.60546 \mathrm{E}+01$ \\
\hline$Z r-91$ & $0.00000 \mathrm{E}+00$ & $0.00000 \mathrm{E}+00$ & $0.00000 \mathrm{E}+00$ & $1.86281 \mathrm{E}+03$ & $1.87115 \mathrm{E}+03$ & $1.85537 \mathrm{E}+03$ \\
\hline$Z r-92$ & $0.00000 \mathrm{E}+00$ & $0.00000 \mathrm{E}+00$ & $0.00000 \mathrm{E}+00$ & $2.12958 \mathrm{E}+03$ & $2.13848 \mathrm{E}+03$ & $2.12374 \mathrm{E}+03$ \\
\hline$Z r-93$ & $0.00000 \mathrm{E}+00$ & $0.00000 \mathrm{E}+00$ & $0.00000 \mathrm{E}+00$ & $2.27885 \mathrm{E}+03$ & $2.28581 \mathrm{E}+03$ & $2.27312 \mathrm{E}+03$ \\
\hline$Z r-94$ & $0.00000 \mathrm{E}+00$ & $0.00000 \mathrm{E}+00$ & $0.00000 \mathrm{E}+00$ & $2.47519 \mathrm{E}+03$ & $2.48228 \mathrm{E}+03$ & $2.47007 \mathrm{E}+03$ \\
\hline$Z r-95$ & $0.00000 \mathrm{E}+00$ & $0.00000 \mathrm{E}+00$ & $0.00000 \mathrm{E}+00$ & $2.26721 \mathrm{E}+02$ & $2.27286 \mathrm{E}+02$ & $2.27188 \mathrm{E}+02$ \\
\hline$Z r-96$ & $0.00000 \mathrm{E}+00$ & $0.00000 \mathrm{E}+00$ & $0.00000 \mathrm{E}+00$ & $2.45971 \mathrm{E}+03$ & $2.46335 \mathrm{E}+03$ & $2.45400 \mathrm{E}+03$ \\
\hline $\mathrm{Nb}-93$ & $0.00000 \mathrm{E}+00$ & $0.00000 \mathrm{E}+00$ & $0.00000 \mathrm{E}+00$ & $1.16522 \mathrm{E}-04$ & $1.15861 \mathrm{E}-04$ & $1.16829 \mathrm{E}-04$ \\
\hline$N b-94$ & $0.00000 \mathrm{E}+00$ & $0.00000 \mathrm{E}+00$ & $0.00000 \mathrm{E}+00$ & $1.36083 \mathrm{E}-03$ & $1.36684 \mathrm{E}-03$ & $1.43592 \mathrm{E}-03$ \\
\hline$N b-95$ & $0.00000 \mathrm{E}+00$ & $0.00000 \mathrm{E}+00$ & $0.00000 \mathrm{E}+00$ & $1.25253 \mathrm{E}+02$ & $1.25580 \mathrm{E}+02$ & $1.25459 \mathrm{E}+02$ \\
\hline Mo-92 & $0.00000 \mathrm{E}+00$ & $0.00000 \mathrm{E}+00$ & $0.00000 \mathrm{E}+00$ & $7.15408 \mathrm{E}-14$ & $7.76367 \mathrm{E}-14$ & 8.59404E-14 \\
\hline Mo-94 & $0.00000 \mathrm{E}+00$ & $0.00000 \mathrm{E}+00$ & $0.00000 \mathrm{E}+00$ & $1.90299 \mathrm{E}-03$ & $2.30225 \mathrm{E}-03$ & $2.36473 \mathrm{E}-03$ \\
\hline Mo-95 & $0.00000 \mathrm{E}+00$ & $0.00000 \mathrm{E}+00$ & $0.00000 \mathrm{E}+00$ & $2.06119 \mathrm{E}+03$ & $2.06316 \mathrm{E}+03$ & $2.05460 \mathrm{E}+03$ \\
\hline Mo-96 & $0.00000 \mathrm{E}+00$ & $0.00000 \mathrm{E}+00$ & $0.00000 \mathrm{E}+00$ & $8.86168 \mathrm{E}+01$ & $9.05719 \mathrm{E}+01$ & $8.89887 \mathrm{E}+01$ \\
\hline Mo-97 & $0.00000 \mathrm{E}+00$ & $0.00000 \mathrm{E}+00$ & $0.00000 \mathrm{E}+00$ & $2.39950 \mathrm{E}+03$ & $2.40035 \mathrm{E}+03$ & $2.39515 \mathrm{E}+03$ \\
\hline Mo-98 & $0.00000 \mathrm{E}+00$ & $0.00000 \mathrm{E}+00$ & $0.00000 \mathrm{E}+00$ & $2.39488 \mathrm{E}+03$ & $2.39519 \mathrm{E}+03$ & $2.39233 \mathrm{E}+03$ \\
\hline Mo-99 & $0.00000 \mathrm{E}+00$ & $0.00000 \mathrm{E}+00$ & $0.00000 \mathrm{E}+00$ & $9.84129 \mathrm{E}+00$ & $9.82441 \mathrm{E}+00$ & $9.81641 \mathrm{E}+00$ \\
\hline Mo- 100 & $0.00000 \mathrm{E}+00$ & $0.00000 \mathrm{E}+00$ & $0.00000 \mathrm{E}+00$ & $2.66019 \mathrm{E}+03$ & $2.65730 \mathrm{E}+03$ & $2.65551 \mathrm{E}+03$ \\
\hline Tc-99 & $0.00000 \mathrm{E}+00$ & $0.00000 \mathrm{E}+00$ & $0.00000 \mathrm{E}+00$ & $2.29994 \mathrm{E}+03$ & $2.30317 \mathrm{E}+03$ & $2.30152 \mathrm{E}+03$ \\
\hline$R u-96$ & $0.00000 \mathrm{E}+00$ & $0.00000 \mathrm{E}+00$ & $0.00000 \mathrm{E}+00$ & $0.00000 \mathrm{E}+00$ & $0.00000 \mathrm{E}+00$ & $0.00000 \mathrm{E}+00$ \\
\hline$R u-98$ & $0.00000 \mathrm{E}+00$ & $0.00000 \mathrm{E}+00$ & $0.00000 \mathrm{E}+00$ & $1.47383 \mathrm{E}-07$ & $1.78547 \mathrm{E}-07$ & $1.84935 \mathrm{E}-07$ \\
\hline$R u-99$ & $0.00000 \mathrm{E}+00$ & $0.00000 \mathrm{E}+00$ & $0.00000 \mathrm{E}+00$ & $8.90960 \mathrm{E}-02$ & $8.90380 \mathrm{E}-02$ & $8.89327 \mathrm{E}-02$ \\
\hline
\end{tabular}




\begin{tabular}{|c|c|c|c|c|c|c|}
\hline \multirow[b]{2}{*}{ Nuclide } & \multicolumn{3}{|c|}{ Fresh Fuel } & \multicolumn{3}{|c|}{ Discharge } \\
\hline & Unit Cell & $\begin{array}{l}\text { Full Core } \\
\text { (NO LBP) }\end{array}$ & Full Core (3) & Unit Cell & $\begin{array}{l}\text { Full Core } \\
\text { (NO LBP) }\end{array}$ & Full Core (3) \\
\hline$R u-100$ & $0.00000 \mathrm{E}+00$ & $0.00000 \mathrm{E}+00$ & $0.00000 \mathrm{E}+00$ & $2.58870 \mathrm{E}+02$ & $2.54448 \mathrm{E}+02$ & $2.53483 \mathrm{E}+02$ \\
\hline$R u-101$ & $0.00000 \mathrm{E}+00$ & $0.00000 \mathrm{E}+00$ & $0.00000 \mathrm{E}+00$ & $2.17074 \mathrm{E}+03$ & $2.16529 \mathrm{E}+03$ & $2.16597 \mathrm{E}+03$ \\
\hline$R u-102$ & $0.00000 \mathrm{E}+00$ & $0.00000 \mathrm{E}+00$ & $0.00000 \mathrm{E}+00$ & $2.04782 \mathrm{E}+03$ & $2.04160 \mathrm{E}+03$ & $2.04838 \mathrm{E}+03$ \\
\hline$R u-103$ & $0.00000 \mathrm{E}+00$ & $0.00000 \mathrm{E}+00$ & $0.00000 \mathrm{E}+00$ & $1.03070 \mathrm{E}+02$ & $1.02104 \mathrm{E}+02$ & $.01869 \mathrm{E}+02$ \\
\hline Ru-104 & $0.00000 \mathrm{E}+00$ & $0.00000 \mathrm{E}+00$ & $0.00000 \mathrm{E}+00$ & $1.18535 \mathrm{E}+03$ & $1.17045 \mathrm{E}+03$ & $1.19104 \mathrm{E}+03$ \\
\hline$R u-105$ & $0.00000 \mathrm{E}+00$ & $0.00000 \mathrm{E}+00$ & $0.00000 \mathrm{E}+00$ & $2.93347 \mathrm{E}-01$ & $2.88539 \mathrm{E}-01$ & $2.86109 \mathrm{E}-01$ \\
\hline$R u-106$ & $0.00000 \mathrm{E}+00$ & $0.00000 \mathrm{E}+00$ & $0.00000 \mathrm{E}+00$ & $2.95849 \mathrm{E}+02$ & $2.88075 \mathrm{E}+02$ & $2.95673 \mathrm{E}+02$ \\
\hline$R h-103$ & $0.00000 \mathrm{E}+00$ & $0.00000 \mathrm{E}+00$ & $0.00000 \mathrm{E}+00$ & $1.02065 \mathrm{E}+03$ & $1.02091 \mathrm{E}+03$ & $.03863 \mathrm{E}+03$ \\
\hline$R h-105$ & $0.00000 \mathrm{E}+00$ & $0.00000 \mathrm{E}+00$ & $0.00000 \mathrm{E}+00$ & $2.09436 \mathrm{E}+00$ & $2.05600 \mathrm{E}+00$ & $2.04672 \mathrm{E}+00$ \\
\hline$P d-102$ & & $0.00000 \mathrm{E}+00$ & $0.00000 \mathrm{E}+00$ & $4.64700 \mathrm{E}-05$ & $5.59364 \mathrm{E}-05$ & $6.06464 \mathrm{E}-05$ \\
\hline$P d-104$ & & $0.00000 \mathrm{E}+00$ & $0.00000 \mathrm{E}+00$ & & $4.88486 \mathrm{E}+02$ & $87619 \mathrm{E}+02$ \\
\hline$P d-105$ & $0.00000 \mathrm{E}+00$ & $0.00000 \mathrm{E}+00$ & $0.00000 \mathrm{E}+00$ & $7.37364 \mathrm{E}+02$ & $7.22673 \mathrm{E}+02$ & $.45295 \mathrm{E}+02$ \\
\hline$P d-106$ & & $0.00000 \mathrm{E}+00$ & $0.00000 \mathrm{E}+00$ & $3.54818 \mathrm{E}+02$ & $3.47453 \mathrm{E}+02$ & $.61762 \mathrm{E}+02$ \\
\hline$P d-107$ & $0.00000 \mathrm{E}+00$ & $0.00000 \mathrm{E}+00$ & $0.00000 \mathrm{E}+00$ & $3.64354 \mathrm{E}+02$ & $3.52497 \mathrm{E}+02$ & $3.69639 \mathrm{E}+02$ \\
\hline$P d-108$ & $0.00000 \mathrm{E}+00$ & $0.00000 \mathrm{E}+00$ & $0.00000 \mathrm{E}+00$ & $2.36923 \mathrm{E}+02$ & $2.28751 \mathrm{E}+02$ & $2.41042 \mathrm{E}+02$ \\
\hline$P d-110$ & $0.00000 \mathrm{E}+00$ & $0.00000 \mathrm{E}+00$ & $0.00000 \mathrm{E}+00$ & $8.04749 \mathrm{E}+01$ & $7.77368 \mathrm{E}+01$ & $8.17687 \mathrm{E}+01$ \\
\hline Ag-107 & $0.00000 \mathrm{E}+00$ & $0.00000 \mathrm{E}+00$ & $0.00000 \mathrm{E}+00$ & $3.83569 \mathrm{E}-05$ & $3.70515 \mathrm{E}-05$ & $3.99326 \mathrm{E}-05$ \\
\hline Ag-109 & $0.00000 \mathrm{E}+00$ & $0.00000 \mathrm{E}+00$ & $0.00000 \mathrm{E}+00$ & $9.38409 \mathrm{E}+01$ & $9.13262 \mathrm{E}+01$ & $9.60592 \mathrm{E}+01$ \\
\hline Ag-111 & $0.00000 \mathrm{E}+00$ & $0.00000 \mathrm{E}+00$ & $0.00000 \mathrm{E}+00$ & 7.39584E-01 & $7.22497 \mathrm{E}-01$ & $9471 \mathrm{E}-01$ \\
\hline $\mathrm{Ag}-110 \mathrm{~m}$ & $0.00000 \mathrm{E}+00$ & $0.00000 \mathrm{E}+00$ & $0.00000 \mathrm{E}+00$ & $1.69072 \mathrm{E}+01$ & $1.60310 \mathrm{E}+01$ & $1.67683 \mathrm{E}+01$ \\
\hline$C d-106$ & $0.00000 \mathrm{E}+00$ & $0.00000 \mathrm{E}+00$ & $0.00000 \mathrm{E}+00$ & $2.63565 \mathrm{E}-12$ & $2.56564 \mathrm{E}-12$ & $2.72465 \mathrm{E}-12$ \\
\hline$C d-108$ & $0.00000 \mathrm{E}+00$ & $0.00000 \mathrm{E}+00$ & $0.00000 \mathrm{E}+00$ & $3.21728 \mathrm{E}-05$ & $3.65718 \mathrm{E}-05$ & $4 \mathrm{E}-05$ \\
\hline$C d-110$ & $0.00000 \mathrm{E}+00$ & $0.00000 \mathrm{E}+00$ & $0.00000 \mathrm{E}+00$ & $1.49158 \mathrm{E}+01$ & $1.40186 \mathrm{E}+01$ & $5 \mathrm{E}+01$ \\
\hline$C d-111$ & $0.00000 \mathrm{E}+00$ & $0.00000 \mathrm{E}+00$ & $0.00000 \mathrm{E}+00$ & $3.90538 \mathrm{E}+01$ & $3.78129 \mathrm{E}+01$ & $.96766 \mathrm{E}+01$ \\
\hline Cd-112 & $0.00000 \mathrm{E}+00$ & $0.00000 \mathrm{E}+00$ & $0.00000 \mathrm{E}+00$ & $1.91357 \mathrm{E}+01$ & $1.86666 \mathrm{E}+01$ & $.94270 \mathrm{E}+01$ \\
\hline$C d-113$ & $0.00000 \mathrm{E}+00$ & $0.00000 \mathrm{E}+00$ & $0.00000 \mathrm{E}+00$ & $1.07682 \mathrm{E}-01$ & $1.02447 \mathrm{E}-01$ & $1.05897 \mathrm{E}-01$ \\
\hline Cd-114 & $0.00000 \mathrm{E}+00$ & $0.00000 \mathrm{E}+00$ & $0.00000 \mathrm{E}+00$ & $2.49381 \mathrm{E}+01$ & $2.44859 \mathrm{E}+01$ & $2.52113 \mathrm{E}+01$ \\
\hline$C d-116$ & $0.00000 \mathrm{E}+00$ & $0.00000 \mathrm{E}+00$ & $0.00000 \mathrm{E}+00$ & $9.79022 \mathrm{E}+00$ & $9.69268 \mathrm{E}+00$ & $9.87112 \mathrm{E}+00$ \\
\hline$C d-115 m$ & $0.00000 \mathrm{E}+00$ & $0.00000 \mathrm{E}+00$ & $0.00000 \mathrm{E}+00$ & $3.99443 \mathrm{E}-02$ & $3.95735 \mathrm{E}-02$ & 3.94463E-02 \\
\hline $\operatorname{In}-113$ & $0.00000 \mathrm{E}+00$ & $0.00000 \mathrm{E}+00$ & $0.00000 \mathrm{E}+00$ & & $7.96062 \mathrm{E}-03$ & $8.31685 \mathrm{E}-03$ \\
\hline In-115 & $0.00000 \mathrm{E}+00$ & $0.00000 \mathrm{E}+00$ & $0.00000 \mathrm{E}+00$ & $3.79321 \mathrm{E}+00$ & $3.85011 \mathrm{E}+00$ & $3.93234 \mathrm{E}+00$ \\
\hline Sn-112 & $0.00000 \mathrm{E}+00$ & $0.00000 \mathrm{E}+00$ & $0.00000 \mathrm{E}+00$ & $7.31278 \mathrm{E}-10$ & $8.41390 \mathrm{E}-10$ & $9.13007 \mathrm{E}-10$ \\
\hline Sn-113 & $0.00000 \mathrm{E}+00$ & $0.00000 \mathrm{E}+00$ & $0.00000 \mathrm{E}+00$ & $2.03246 \mathrm{E}-11$ & $2.28355 \mathrm{E}-11$ & $2.43993 \mathrm{E}-11$ \\
\hline Sn-114 & $0.00000 \mathrm{E}+00$ & $0.00000 \mathrm{E}+00$ & $0.00000 \mathrm{E}+00$ & $5.12472 \mathrm{E}-04$ & $4.99278 \mathrm{E}-04$ & $5.15347 \mathrm{E}-04$ \\
\hline$S n-115$ & $0.00000 \mathrm{E}+00$ & $0.00000 \mathrm{E}+00$ & $0.00000 \mathrm{E}+00$ & $4.06970 \mathrm{E}-01$ & 4.02754E-01 & 4.11080E-01 \\
\hline$S n-116$ & $0.00000 \mathrm{E}+00$ & $0.00000 \mathrm{E}+00$ & $0.00000 \mathrm{E}+00$ & $4.95756 \mathrm{E}+00$ & $4.82170 \mathrm{E}+00$ & $4.90003 \mathrm{E}+00$ \\
\hline
\end{tabular}




\section{Depletion Analysis of Modular High Temperature Gas-cooled Reactor Loaded with LEU/Thorium Fuel}

February 2012

\begin{tabular}{|c|c|c|c|c|c|c|}
\hline \multirow[b]{2}{*}{ Nuclide } & \multicolumn{3}{|c|}{ Fresh Fuel } & \multicolumn{3}{|c|}{ Discharge } \\
\hline & Unit Cell & $\begin{array}{l}\text { Full Core } \\
(N O L B P)\end{array}$ & Full Core (3) & Unit Cell & $\begin{array}{l}\text { Full Core } \\
\text { (NO LBP) }\end{array}$ & Full Core (3) \\
\hline Sn-117 & $0.00000 \mathrm{E}+00$ & $0.00000 \mathrm{E}+00$ & $0.00000 \mathrm{E}+00$ & $9.14585 \mathrm{E}+00$ & $9.06849 \mathrm{E}+00$ & $9.22180 \mathrm{E}+00$ \\
\hline$S n-118$ & $0.00000 \mathrm{E}+00$ & $0.00000 \mathrm{E}+00$ & $0.00000 \mathrm{E}+00$ & $7.88328 \mathrm{E}+00$ & $7.83721 \mathrm{E}+00$ & $7.95355 \mathrm{E}+00$ \\
\hline Sn-119 & $0.00000 \mathrm{E}+00$ & $0.00000 \mathrm{E}+00$ & $0.00000 \mathrm{E}+00$ & $8.54659 \mathrm{E}+00$ & $8.50402 \mathrm{E}+00$ & $8.61175 \mathrm{E}+00$ \\
\hline$S n-120$ & $0.00000 \mathrm{E}+00$ & $0.00000 \mathrm{E}+00$ & $0.00000 \mathrm{E}+00$ & $8.60339 \mathrm{E}+00$ & $8.56522 \mathrm{E}+00$ & $8.67487 \mathrm{E}+00$ \\
\hline$S n-122$ & $0.00000 \mathrm{E}+00$ & $0.00000 \mathrm{E}+00$ & $0.00000 \mathrm{E}+00$ & $1.16832 \mathrm{E}+01$ & $1.16234 \mathrm{E}+01$ & $1.17989 \mathrm{E}+01$ \\
\hline$S n-123$ & $0.00000 \mathrm{E}+00$ & $0.00000 \mathrm{E}+00$ & $0.00000 \mathrm{E}+00$ & $1.19924 \mathrm{E}+00$ & $1.19733 \mathrm{E}+00$ & $1.19893 \mathrm{E}+00$ \\
\hline Sn-124 & $0.00000 \mathrm{E}+00$ & $0.00000 \mathrm{E}+00$ & $0.00000 \mathrm{E}+00$ & $2.04744 \mathrm{E}+01$ & $2.03709 \mathrm{E}+01$ & $2.06683 \mathrm{E}+01$ \\
\hline$S n-125$ & $0.00000 \mathrm{E}+00$ & $0.00000 \mathrm{E}+00$ & $0.00000 \mathrm{E}+00$ & $3.13133 \mathrm{E}-01$ & $3.12914 \mathrm{E}-01$ & $.09726 \mathrm{E}-01$ \\
\hline$S n-126$ & $0.00000 \mathrm{E}+00$ & $0.00000 \mathrm{E}+00$ & $0.00000 \mathrm{E}+00$ & $4.91481 \mathrm{E}+01$ & $4.88360 \mathrm{E}+01$ & $4.97162 \mathrm{E}+01$ \\
\hline$S b-121$ & $0.00000 \mathrm{E}+00$ & $0.00000 \mathrm{E}+00$ & $0.00000 \mathrm{E}+00$ & $8.43158 \mathrm{E}+00$ & $8.38577 \mathrm{E}+00$ & $8.50672 \mathrm{E}+00$ \\
\hline$S b-123$ & $0.00000 \mathrm{E}+00$ & $0.00000 \mathrm{E}+00$ & $0.00000 \mathrm{E}+00$ & $1.10054 \mathrm{E}+01$ & $1.09619 \mathrm{E}+01$ & $1.11507 \mathrm{E}+01$ \\
\hline$S b-124$ & $0.00000 \mathrm{E}+00$ & $0.00000 \mathrm{E}+00$ & $0.00000 \mathrm{E}+00$ & $6.89383 \mathrm{E}-02$ & $6.94845 \mathrm{E}-02$ & $.86436 \mathrm{E}-02$ \\
\hline$S b-125$ & $0.00000 \mathrm{E}+00$ & $0.00000 \mathrm{E}+00$ & $0.00000 \mathrm{E}+00$ & $2.07168 \mathrm{E}+01$ & $2.05927 \mathrm{E}+01$ & $2.08933 \mathrm{E}+01$ \\
\hline$S b-126$ & $0.00000 \mathrm{E}+00$ & $0.00000 \mathrm{E}+00$ & $0.00000 \mathrm{E}+00$ & $3.40267 \mathrm{E}-02$ & 3.44881E-02 & $.40597 \mathrm{E}-02$ \\
\hline Te-120 & $0.00000 \mathrm{E}+00$ & $0.00000 \mathrm{E}+00$ & $0.00000 \mathrm{E}+00$ & $4.28013 \mathrm{E}-10$ & $4.27660 \mathrm{E}-10$ & $47042 \mathrm{E}-10$ \\
\hline $\mathrm{Te}-122$ & $0.00000 \mathrm{E}+00$ & $0.00000 \mathrm{E}+00$ & $0.00000 \mathrm{E}+00$ & 33309E-01 & $15 \mathrm{E}-01$ & $4 \mathrm{E}-01$ \\
\hline$T e-123$ & $0.00000 \mathrm{E}+00$ & $0.00000 \mathrm{E}+00$ & $0.00000 \mathrm{E}+00$ & E-03 & $3.61770 \mathrm{E}-03$ & $.73019 \mathrm{E}-03$ \\
\hline $\mathrm{Te}-124$ & $0.00000 \mathrm{E}+00$ & $0.00000 \mathrm{E}+00$ & $0.00000 \mathrm{E}+00$ & $2.80427 \mathrm{E}-01$ & $2.79184 \mathrm{E}-01$ & $2.87660 \mathrm{E}-01$ \\
\hline$T e-125$ & $0.00000 \mathrm{E}+00$ & $0.00000 \mathrm{E}+00$ & $0.00000 \mathrm{E}+00$ & $6.48419 \mathrm{E}+00$ & $6.43816 \mathrm{E}+00$ & $6.59596 \mathrm{E}+00$ \\
\hline$T e-126$ & $0.00000 \mathrm{E}+00$ & $0.00000 \mathrm{E}+00$ & $0.00000 \mathrm{E}+00$ & $1.73202 \mathrm{E}+00$ & $1.73741 \mathrm{E}+00$ & $1.74725 \mathrm{E}+00$ \\
\hline $\mathrm{Te}-128$ & $0.00000 \mathrm{E}+00$ & $0.00000 \mathrm{E}+00$ & $0.00000 \mathrm{E}+00$ & $2.46658 \mathrm{E}+02$ & $2.45851 \mathrm{E}+02$ & $2.48190 \mathrm{E}+02$ \\
\hline Te-130 & $0.00000 \mathrm{E}+00$ & $0.00000 \mathrm{E}+00$ & $0.00000 \mathrm{E}+00$ & $1.06383 \mathrm{E}+03$ & $1.06220 \mathrm{E}+03$ & $1.06452 \mathrm{E}+03$ \\
\hline Te-132 & $0.00000 \mathrm{E}+00$ & $0.00000 \mathrm{E}+00$ & $0.00000 \mathrm{E}+00$ & $1.17615 \mathrm{E}+01$ & $1.17453 \mathrm{E}+01$ & $1.17214 \mathrm{E}+01$ \\
\hline$T e-127 m$ & $0.00000 \mathrm{E}+00$ & $0.00000 \mathrm{E}+00$ & $0.00000 \mathrm{E}+00$ & $4.90812 \mathrm{E}-01$ & $4.82467 \mathrm{E}-01$ & 4.91507E-01 \\
\hline $\mathrm{Te}-129 \mathrm{~m}$ & $0.00000 \mathrm{E}+00$ & $0.00000 \mathrm{E}+00$ & $0.00000 \mathrm{E}+00$ & $7.85542 \mathrm{E}-02$ & $8.02153 \mathrm{E}-02$ & $8.00459 \mathrm{E}-02$ \\
\hline$I-127$ & $0.00000 \mathrm{E}+00$ & $0.00000 \mathrm{E}+00$ & $0.00000 \mathrm{E}+00$ & $1.21720 \mathrm{E}+02$ & $1.20840 \mathrm{E}+02$ & $1.22881 \mathrm{E}+02$ \\
\hline$I-129$ & $0.00000 \mathrm{E}+00$ & $0.00000 \mathrm{E}+00$ & $0.00000 \mathrm{E}+00$ & $4.02595 \mathrm{E}+02$ & $4.00385 \mathrm{E}+02$ & $4.06088 \mathrm{E}+02$ \\
\hline$I-130$ & $0.00000 \mathrm{E}+00$ & $0.00000 \mathrm{E}+00$ & $0.00000 \mathrm{E}+00$ & $3.28043 \mathrm{E}-02$ & $3.36605 \mathrm{E}-02$ & $3.29082 \mathrm{E}-02$ \\
\hline I-131 & $0.00000 \mathrm{E}+00$ & $0.00000 \mathrm{E}+00$ & $0.00000 \mathrm{E}+00$ & $2.07974 \mathrm{E}+01$ & $2.07680 \mathrm{E}+01$ & $2.07165 \mathrm{E}+01$ \\
\hline$I-135$ & $0.00000 \mathrm{E}+00$ & $0.00000 \mathrm{E}+00$ & $0.00000 \mathrm{E}+00$ & $1.38350 \mathrm{E}+00$ & $1.38008 \mathrm{E}+00$ & $1.37907 \mathrm{E}+00$ \\
\hline$X e-123$ & $0.00000 \mathrm{E}+00$ & $0.00000 \mathrm{E}+00$ & $0.00000 \mathrm{E}+00$ & $0.00000 \mathrm{E}+00$ & $0.00000 \mathrm{E}+00$ & $0.00000 \mathrm{E}+00$ \\
\hline$X e-124$ & $0.00000 \mathrm{E}+00$ & $0.00000 \mathrm{E}+00$ & $0.00000 \mathrm{E}+00$ & $0.00000 \mathrm{E}+00$ & $0.00000 \mathrm{E}+00$ & $0.00000 \mathrm{E}+00$ \\
\hline$X e-126$ & $0.00000 \mathrm{E}+00$ & $0.00000 \mathrm{E}+00$ & $0.00000 \mathrm{E}+00$ & $2.38659 \mathrm{E}-05$ & $2.83160 \mathrm{E}-05$ & $3.02620 \mathrm{E}-05$ \\
\hline$X e-128$ & $0.00000 \mathrm{E}+00$ & $0.00000 \mathrm{E}+00$ & $0.00000 \mathrm{E}+00$ & $5.16077 \mathrm{E}+00$ & $5.24705 \mathrm{E}+00$ & $5.36750 \mathrm{E}+00$ \\
\hline$X e-129$ & $0.00000 \mathrm{E}+00$ & $0.00000 \mathrm{E}+00$ & $0.00000 \mathrm{E}+00$ & $2.90222 \mathrm{E}-02$ & 3.04447E-02 & $3.11773 \mathrm{E}-02$ \\
\hline$X e-130$ & $0.00000 \mathrm{E}+00$ & $0.00000 \mathrm{E}+00$ & $0.00000 \mathrm{E}+00$ & $1.62495 \mathrm{E}+01$ & $1.65502 \mathrm{E}+01$ & $1.63207 \mathrm{E}+01$ \\
\hline
\end{tabular}




\begin{tabular}{|c|c|c|c|c|c|c|}
\hline \multirow[b]{2}{*}{ Nuclide } & \multicolumn{3}{|c|}{ Fresh Fuel } & \multicolumn{3}{|c|}{ Discharge } \\
\hline & Unit Cell & $\begin{array}{l}\text { Full Core } \\
\text { (NO LBP) }\end{array}$ & Full Core (3) & Unit Cell & $\begin{array}{l}\text { Full Core } \\
\text { (NO LBP) }\end{array}$ & Full Core (3) \\
\hline $\mathrm{Xe}-131$ & $0.00000 \mathrm{E}+00$ & $0.00000 \mathrm{E}+00$ & $0.00000 \mathrm{E}+00$ & $1.27757 \mathrm{E}+03$ & $1.27671 \mathrm{E}+03$ & $1.28358 \mathrm{E}+03$ \\
\hline$X e-132$ & $0.00000 \mathrm{E}+00$ & $0.00000 \mathrm{E}+00$ & $0.00000 \mathrm{E}+00$ & $2.98203 \mathrm{E}+03$ & $2.97650 \mathrm{E}+03$ & $2.97949 \mathrm{E}+03$ \\
\hline$X e-133$ & $0.00000 \mathrm{E}+00$ & $0.00000 \mathrm{E}+00$ & $0.00000 \mathrm{E}+00$ & $2.82000 \mathrm{E}+01$ & $2.81556 \mathrm{E}+01$ & $2.81318 \mathrm{E}+01$ \\
\hline $\mathrm{Xe}-134$ & $0.00000 \mathrm{E}+00$ & $0.00000 \mathrm{E}+00$ & $0.00000 \mathrm{E}+00$ & $4.42198 \mathrm{E}+03$ & $4.42030 \mathrm{E}+03$ & $4.41413 \mathrm{E}+03$ \\
\hline $\mathrm{Xe}-135$ & $0.00000 \mathrm{E}+00$ & $0.00000 \mathrm{E}+00$ & $0.00000 \mathrm{E}+00$ & 4.01462E-01 & 3.89374E-01 & 4.00734E-01 \\
\hline$X e-136$ & $0.00000 \mathrm{E}+00$ & $0.00000 \mathrm{E}+00$ & $0.00000 \mathrm{E}+00$ & $6.51528 \mathrm{E}+03$ & $6.52525 \mathrm{E}+03$ & $6.48768 \mathrm{E}+03$ \\
\hline Cs-133 & $0.00000 \mathrm{E}+00$ & $0.00000 \mathrm{E}+00$ & $0.00000 \mathrm{E}+00$ & $3.31112 \mathrm{E}+03$ & $3.31477 \mathrm{E}+03$ & $3.31552 \mathrm{E}+03$ \\
\hline Cs-134 & $0.00000 \mathrm{E}+00$ & $0.00000 \mathrm{E}+00$ & $0.00000 \mathrm{E}+00$ & $3.16884 \mathrm{E}+02$ & $3.12640 \mathrm{E}+02$ & $3.09394 \mathrm{E}+02$ \\
\hline Cs-135 & $0.00000 \mathrm{E}+00$ & $0.00000 \mathrm{E}+00$ & $0.00000 \mathrm{E}+00$ & $1.04410 \mathrm{E}+03$ & $1.02573 \mathrm{E}+03$ & $1.06829 \mathrm{E}+03$ \\
\hline Cs-136 & $0.00000 \mathrm{E}+00$ & $0.00000 \mathrm{E}+00$ & $0.00000 \mathrm{E}+00$ & $1.37278 \mathrm{E}+00$ & $1.39332 \mathrm{E}+00$ & $1.39341 \mathrm{E}+00$ \\
\hline Cs-137 & & $0.00000 \mathrm{E}+00$ & $0000 \mathrm{E}+00$ & $3.66460 \mathrm{E}+03$ & $3.66248 \mathrm{E}+03$ & $2 \mathrm{E}+03$ \\
\hline$B a-130$ & $0.00000 \mathrm{E}+00$ & $0.00000 \mathrm{E}+00$ & $0.00000 \mathrm{E}+00$ & $3.75681 \mathrm{E}-20$ & $5.23224 \mathrm{E}-20$ & $5.79324 \mathrm{E}-20$ \\
\hline$B a-132$ & & $0.00000 \mathrm{E}+00$ & $0.00000 \mathrm{E}+00$ & $2.48004 \mathrm{E}-05$ & $2.94199 \mathrm{E}-05$ & $3.08531 \mathrm{E}-05$ \\
\hline$B a-133$ & $0.00000 \mathrm{E}+00$ & $0.00000 \mathrm{E}+00$ & $0.00000 \mathrm{E}+00$ & $1.65380 \mathrm{E}-05$ & $1.89722 \mathrm{E}-05$ & $2.03551 \mathrm{E}-05$ \\
\hline$B a-134$ & $0.00000 \mathrm{E}+00$ & $0.00000 \mathrm{E}+00$ & $0.00000 \mathrm{E}+00$ & $9.55427 \mathrm{E}+01$ & $9.35936 \mathrm{E}+01$ & $9.56738 \mathrm{E}+01$ \\
\hline$B a-135$ & $0.00000 \mathrm{E}+00$ & $0.00000 \mathrm{E}+00$ & $0.00000 \mathrm{E}+00$ & $4.13473 \mathrm{E}-01$ & 4.22057E-01 & $4.30681 \mathrm{E}-01$ \\
\hline$B a-136$ & $0.00000 \mathrm{E}+00$ & $0.00000 \mathrm{E}+00$ & $0.00000 \mathrm{E}+00$ & $4.14894 \mathrm{E}+01$ & $4.15752 \mathrm{E}+01$ & $4.30956 \mathrm{E}+01$ \\
\hline$B a-137$ & $0.00000 \mathrm{E}+00$ & $0.00000 \mathrm{E}+00$ & $0.00000 \mathrm{E}+00$ & $1.12843 \mathrm{E}+02$ & $1.12757 \mathrm{E}+02$ & $1.12847 \mathrm{E}+02$ \\
\hline$B a-138$ & $0.00000 \mathrm{E}+00$ & $0.00000 \mathrm{E}+00$ & $0.00000 \mathrm{E}+00$ & $3.89708 \mathrm{E}+03$ & $3.89753 \mathrm{E}+03$ & $.89062 \mathrm{E}+03$ \\
\hline$B a-140$ & $0.00000 \mathrm{E}+00$ & $0.00000 \mathrm{E}+00$ & $0.00000 \mathrm{E}+00$ & $6.58738 \mathrm{E}+01$ & $6.58969 \mathrm{E}+01$ & $6.58909 \mathrm{E}+01$ \\
\hline$L a-138$ & $0.00000 \mathrm{E}+00$ & $0.00000 \mathrm{E}+00$ & $0.00000 \mathrm{E}+00$ & $1.91032 \mathrm{E}-02$ & $1.94061 \mathrm{E}-02$ & $1.95889 \mathrm{E}-02$ \\
\hline La-139 & $0.00000 \mathrm{E}+00$ & $0.00000 \mathrm{E}+00$ & $0.00000 \mathrm{E}+00$ & $3.71125 \mathrm{E}+03$ & $3.71201 \mathrm{E}+03$ & $.70661 \mathrm{E}+03$ \\
\hline$L a-140$ & $0.00000 \mathrm{E}+00$ & $0.00000 \mathrm{E}+00$ & $0.00000 \mathrm{E}+00$ & $9.00911 \mathrm{E}+00$ & $9.02118 \mathrm{E}+00$ & $.00888 \mathrm{E}+00$ \\
\hline $\mathrm{Ce}-136$ & $0.00000 \mathrm{E}+00$ & $0.00000 \mathrm{E}+00$ & $0.00000 \mathrm{E}+00$ & $0.00000 \mathrm{E}+00$ & $0.00000 \mathrm{E}+00$ & $0.00000 \mathrm{E}+00$ \\
\hline $\mathrm{Ce}-138$ & $0.00000 \mathrm{E}+00$ & $0.00000 \mathrm{E}+00$ & $0.00000 \mathrm{E}+00$ & $9.95959 \mathrm{E}-07$ & $9.74965 \mathrm{E}-07$ & $1.01274 \mathrm{E}-06$ \\
\hline $\mathrm{Ce}-139$ & $0.00000 \mathrm{E}+00$ & $0.00000 \mathrm{E}+00$ & $0.00000 \mathrm{E}+00$ & 5.01557E-04 & $5.89059 \mathrm{E}-04$ & 6.00679E-04 \\
\hline $\mathrm{Ce}-140$ & $0.00000 \mathrm{E}+00$ & $0.00000 \mathrm{E}+00$ & $0.00000 \mathrm{E}+00$ & $3.61135 \mathrm{E}+03$ & $3.61476 \mathrm{E}+03$ & $3.60606 \mathrm{E}+03$ \\
\hline $\mathrm{Ce}-141$ & $0.00000 \mathrm{E}+00$ & $0.00000 \mathrm{E}+00$ & $0.00000 \mathrm{E}+00$ & $1.63034 \mathrm{E}+02$ & $1.63204 \mathrm{E}+02$ & $1.63184 \mathrm{E}+02$ \\
\hline $\mathrm{Ce}-142$ & $0.00000 \mathrm{E}+00$ & $0.00000 \mathrm{E}+00$ & $0.00000 \mathrm{E}+00$ & $3.47678 \mathrm{E}+03$ & $3.48054 \mathrm{E}+03$ & $3.47330 \mathrm{E}+03$ \\
\hline $\mathrm{Ce}-143$ & $0.00000 \mathrm{E}+00$ & $0.00000 \mathrm{E}+00$ & $0.00000 \mathrm{E}+00$ & $6.50581 \mathrm{E}+00$ & $6.51333 \mathrm{E}+00$ & $6.51366 \mathrm{E}+00$ \\
\hline $\mathrm{Ce}-144$ & $0.00000 \mathrm{E}+00$ & $0.00000 \mathrm{E}+00$ & $0.00000 \mathrm{E}+00$ & $1.18351 \mathrm{E}+03$ & $1.18594 \mathrm{E}+03$ & $1.18245 \mathrm{E}+03$ \\
\hline Pr-141 & $0.00000 \mathrm{E}+00$ & $0.00000 \mathrm{E}+00$ & $0.00000 \mathrm{E}+00$ & $3.23750 \mathrm{E}+03$ & $3.23847 \mathrm{E}+03$ & $3.23423 \mathrm{E}+03$ \\
\hline $\operatorname{Pr}-142$ & $0.00000 \mathrm{E}+00$ & $0.00000 \mathrm{E}+00$ & $0.00000 \mathrm{E}+00$ & $1.69952 \mathrm{E}-01$ & $1.75813 \mathrm{E}-01$ & 1.69335E-01 \\
\hline Pr-143 & $0.00000 \mathrm{E}+00$ & $0.00000 \mathrm{E}+00$ & $0.00000 \mathrm{E}+00$ & $6.50694 \mathrm{E}+01$ & $6.51593 \mathrm{E}+01$ & $6.51931 \mathrm{E}+01$ \\
\hline$N d-142$ & $0.00000 \mathrm{E}+00$ & $0.00000 \mathrm{E}+00$ & $0.00000 \mathrm{E}+00$ & $5.51426 \mathrm{E}+01$ & $5.67091 \mathrm{E}+01$ & $5.49398 \mathrm{E}+01$ \\
\hline$N d-143$ & $0.00000 \mathrm{E}+00$ & $0.00000 \mathrm{E}+00$ & $0.00000 \mathrm{E}+00$ & $2.34074 \mathrm{E}+03$ & $2.32701 \mathrm{E}+03$ & $2.35226 \mathrm{E}+03$ \\
\hline
\end{tabular}




\section{Depletion Analysis of Modular High Temperature Gas-cooled Reactor Loaded with LEU/Thorium Fuel}

February 2012

\begin{tabular}{|c|c|c|c|c|c|c|}
\hline \multirow[b]{2}{*}{ Nuclide } & \multicolumn{3}{|c|}{ Fresh Fuel } & \multicolumn{3}{|c|}{ Discharge } \\
\hline & Unit Cell & $\begin{array}{l}\text { Full Core } \\
(N O L B P)\end{array}$ & Full Core (3) & Unit Cell & $\begin{array}{l}\text { Full Core } \\
\text { (NO LBP) }\end{array}$ & Full Core (3) \\
\hline$N d-144$ & $0.00000 \mathrm{E}+00$ & $.00000 \mathrm{E}+00$ & $0.00000 \mathrm{E}+00$ & $3.00554 \mathrm{E}+03$ & $3.02789 \mathrm{E}+03$ & $2.97947 \mathrm{E}+03$ \\
\hline$N d-145$ & $0.00000 \mathrm{E}+00$ & $0.00000 \mathrm{E}+00$ & $0.00000 \mathrm{E}+00$ & $2.07633 \mathrm{E}+03$ & $2.07847 \mathrm{E}+03$ & $2.07454 \mathrm{E}+03$ \\
\hline$N d-146$ & $0.00000 \mathrm{E}+00$ & $0.00000 \mathrm{E}+00$ & $0.00000 \mathrm{E}+00$ & $2.04248 \mathrm{E}+03$ & $2.04528 \mathrm{E}+03$ & $2.03554 \mathrm{E}+03$ \\
\hline$N d-147$ & $0.00000 \mathrm{E}+00$ & $0.00000 \mathrm{E}+00$ & $0.00000 \mathrm{E}+00$ & $2.05982 \mathrm{E}+01$ & $2.05695 \mathrm{E}+01$ & $2.05858 \mathrm{E}+01$ \\
\hline$N d-148$ & $0.00000 \mathrm{E}+00$ & $0.00000 \mathrm{E}+00$ & $0.00000 \mathrm{E}+00$ & $1.06003 \mathrm{E}+03$ & $1.05940 \mathrm{E}+03$ & $1.05799 \mathrm{E}+03$ \\
\hline$N d-150$ & $0.00000 \mathrm{E}+00$ & $0.00000 \mathrm{E}+00$ & $0.00000 \mathrm{E}+00$ & $4.54435 \mathrm{E}+02$ & $4.52508 \mathrm{E}+02$ & $4.54688 \mathrm{E}+02$ \\
\hline$P m-147$ & $0.00000 \mathrm{E}+00$ & $0.00000 \mathrm{E}+00$ & $0.00000 \mathrm{E}+00$ & $5.62171 \mathrm{E}+02$ & $5.67535 \mathrm{E}+02$ & $5.70387 \mathrm{E}+02$ \\
\hline Pm-148 & $0.00000 \mathrm{E}+00$ & $0.00000 \mathrm{E}+00$ & $0.00000 \mathrm{E}+00$ & $3.48024 \mathrm{E}+00$ & $3.47260 \mathrm{E}+00$ & $3.37897 \mathrm{E}+00$ \\
\hline Pm-149 & $0.00000 \mathrm{E}+00$ & $0.00000 \mathrm{E}+00$ & $0.00000 \mathrm{E}+00$ & $3.49074 \mathrm{E}+00$ & $3.48848 \mathrm{E}+00$ & $44228 \mathrm{E}+00$ \\
\hline Pm-151 & $0.00000 \mathrm{E}+00$ & $0.00000 \mathrm{E}+00$ & $0.00000 \mathrm{E}+00$ & $5.74879 \mathrm{E}-01$ & $5.71216 \mathrm{E}-01$ & $.69422 \mathrm{E}-01$ \\
\hline$P m-148 m$ & $0.00000 \mathrm{E}+00$ & $0.00000 \mathrm{E}+00$ & $0.00000 \mathrm{E}+00$ & $2.75869 \mathrm{E}+00$ & $2.67950 \mathrm{E}+00$ & $2.68960 \mathrm{E}+00$ \\
\hline Sm-144 & $0.00000 \mathrm{E}+00$ & $0.00000 \mathrm{E}+00$ & $0.00000 \mathrm{E}+00$ & $9.19900 \mathrm{E}-16$ & $97780 \mathrm{E}-16$ & $.95930 \mathrm{E}-16$ \\
\hline$S m-147$ & $0.00000 \mathrm{E}+00$ & $0.00000 \mathrm{E}+00$ & $0.00000 \mathrm{E}+00$ & $2.20330 \mathrm{E}+02$ & $2.22007 \mathrm{E}+02$ & $2.20673 \mathrm{E}+02$ \\
\hline$S m-148$ & $0.00000 \mathrm{E}+00$ & $0.00000 \mathrm{E}+00$ & $0.00000 \mathrm{E}+00$ & $3.27413 \mathrm{E}+02$ & $3.22802 \mathrm{E}+02$ & $3.21983 \mathrm{E}+02$ \\
\hline$S m-149$ & $0.00000 \mathrm{E}+00$ & $0.00000 \mathrm{E}+00$ & $0.00000 \mathrm{E}+00$ & $4.44213 \mathrm{E}+00$ & $4.28667 \mathrm{E}+00$ & $4.39017 \mathrm{E}+00$ \\
\hline$S m-150$ & $0.00000 \mathrm{E}+00$ & $0.00000 \mathrm{E}+00$ & $0.00000 \mathrm{E}+00$ & $7.86336 \mathrm{E}+02$ & $7.82452 \mathrm{E}+02$ & $7.82818 \mathrm{E}+02$ \\
\hline$S m-151$ & $0.00000 \mathrm{E}+00$ & $0.00000 \mathrm{E}+00$ & $0.00000 \mathrm{E}+00$ & $2.80644 \mathrm{E}+01$ & $2.74373 \mathrm{E}+01$ & $2.79832 \mathrm{E}+01$ \\
\hline$S m-152$ & $0.00000 \mathrm{E}+00$ & $0.00000 \mathrm{E}+00$ & $0.00000 \mathrm{E}+00$ & $3.15990 \mathrm{E}+02$ & $3.16923 \mathrm{E}+02$ & $3.19383 \mathrm{E}+02$ \\
\hline$S m-153$ & $0.00000 \mathrm{E}+00$ & $0.00000 \mathrm{E}+00$ & $0.00000 \mathrm{E}+00$ & $2.39474 \mathrm{E}+00$ & $2.39737 \mathrm{E}+00$ & $2.34356 \mathrm{E}+00$ \\
\hline$S m-154$ & $0.00000 \mathrm{E}+00$ & $0.00000 \mathrm{E}+00$ & $0.00000 \mathrm{E}+00$ & $7.70156 \mathrm{E}+01$ & $7.57989 \mathrm{E}+01$ & $7.72546 \mathrm{E}+01$ \\
\hline$E u-151$ & $0.00000 \mathrm{E}+00$ & $0.00000 \mathrm{E}+00$ & $0.00000 \mathrm{E}+00$ & $1.82953 \mathrm{E}-02$ & $1.76097 \mathrm{E}-02$ & $1.86791 \mathrm{E}-02$ \\
\hline$E u-152$ & $0.00000 \mathrm{E}+00$ & $0.00000 \mathrm{E}+00$ & $0.00000 \mathrm{E}+00$ & $3.03317 \mathrm{E}-02$ & $2.87743 \mathrm{E}-02$ & $3.07808 \mathrm{E}-02$ \\
\hline$E u-153$ & $0.00000 \mathrm{E}+00$ & $0.00000 \mathrm{E}+00$ & $0.00000 \mathrm{E}+00$ & $2.86863 \mathrm{E}+02$ & $2.84438 \mathrm{E}+02$ & $2.85519 \mathrm{E}+02$ \\
\hline$E u-154$ & $0.00000 \mathrm{E}+00$ & $0.00000 \mathrm{E}+00$ & $0.00000 \mathrm{E}+00$ & $3.65575 \mathrm{E}+01$ & $3.56739 \mathrm{E}+01$ & $3.58260 \mathrm{E}+01$ \\
\hline$E u-155$ & $0.00000 \mathrm{E}+00$ & $0.00000 \mathrm{E}+00$ & $0.00000 \mathrm{E}+00$ & $1.34626 \mathrm{E}+01$ & $1.35306 \mathrm{E}+01$ & $1.36104 \mathrm{E}+01$ \\
\hline$E u-156$ & $0.00000 \mathrm{E}+00$ & $0.00000 \mathrm{E}+00$ & $0.00000 \mathrm{E}+00$ & $9.90359 \mathrm{E}+00$ & $9.89226 \mathrm{E}+00$ & $9.64036 \mathrm{E}+00$ \\
\hline Eu-157 & $0.00000 \mathrm{E}+00$ & $0.00000 \mathrm{E}+00$ & $0.00000 \mathrm{E}+00$ & $3.26846 \mathrm{E}-02$ & $3.20608 \mathrm{E}-02$ & $3.12330 \mathrm{E}-02$ \\
\hline$G d-152$ & $0.00000 \mathrm{E}+00$ & $0.00000 \mathrm{E}+00$ & $0.00000 \mathrm{E}+00$ & $5.88689 \mathrm{E}-04$ & $5.60354 \mathrm{E}-04$ & $6.27256 \mathrm{E}-04$ \\
\hline$G d-153$ & $0.00000 \mathrm{E}+00$ & $0.00000 \mathrm{E}+00$ & $0.00000 \mathrm{E}+00$ & $5.73646 \mathrm{E}-05$ & $5.46524 \mathrm{E}-05$ & $.09728 \mathrm{E}-05$ \\
\hline$G d-154$ & $0.00000 \mathrm{E}+00$ & $0.00000 \mathrm{E}+00$ & $0.00000 \mathrm{E}+00$ & $2.64048 \mathrm{E}+00$ & $2.56104 \mathrm{E}+00$ & $2.65766 \mathrm{E}+00$ \\
\hline$G d-155$ & $0.00000 \mathrm{E}+00$ & $0.00000 \mathrm{E}+00$ & $0.00000 \mathrm{E}+00$ & $8.41099 \mathrm{E}-02$ & $8.17998 \mathrm{E}-02$ & $8.53847 \mathrm{E}-02$ \\
\hline$G d-156$ & $0.00000 \mathrm{E}+00$ & $0.00000 \mathrm{E}+00$ & $0.00000 \mathrm{E}+00$ & $1.56625 \mathrm{E}+02$ & $1.54791 \mathrm{E}+02$ & $1.55780 \mathrm{E}+02$ \\
\hline$G d-157$ & $0.00000 \mathrm{E}+00$ & $0.00000 \mathrm{E}+00$ & $0.00000 \mathrm{E}+00$ & $1.49015 \mathrm{E}-01$ & $1.42776 \mathrm{E}-01$ & $1.44389 \mathrm{E}-01$ \\
\hline$G d-158$ & $0.00000 \mathrm{E}+00$ & $0.00000 \mathrm{E}+00$ & $0.00000 \mathrm{E}+00$ & $2.94699 \mathrm{E}+01$ & $2.87242 \mathrm{E}+01$ & $2.97085 \mathrm{E}+01$ \\
\hline$G d-160$ & $0.00000 \mathrm{E}+00$ & $0.00000 \mathrm{E}+00$ & $0.00000 \mathrm{E}+00$ & $1.73022 \mathrm{E}+00$ & $1.67019 \mathrm{E}+00$ & $1.76015 \mathrm{E}+00$ \\
\hline$T b-159$ & $0.00000 \mathrm{E}+00$ & $0.00000 \mathrm{E}+00$ & $0.00000 \mathrm{E}+00$ & $3.98594 \mathrm{E}+00$ & $3.86225 \mathrm{E}+00$ & $4.05502 \mathrm{E}+00$ \\
\hline
\end{tabular}




\begin{tabular}{|c|c|c|c|c|c|c|}
\hline \multirow[b]{2}{*}{ Nuclide } & \multicolumn{3}{|c|}{ Fresh Fuel } & \multicolumn{3}{|c|}{ Discharge } \\
\hline & Unit Cell & $\begin{array}{l}\text { Full Core } \\
\text { (NO LBP) }\end{array}$ & Full Core (3) & Unit Cell & $\begin{array}{l}\text { Full Core } \\
(N O L B P)\end{array}$ & Full Core (3) \\
\hline$T b-160$ & $0.00000 \mathrm{E}+00$ & $0.00000 \mathrm{E}+00$ & $0.00000 \mathrm{E}+00$ & $8.45852 \mathrm{E}-02$ & $8.32718 \mathrm{E}-02$ & $8.58421 \mathrm{E}-02$ \\
\hline$D y-156$ & $0.00000 \mathrm{E}+00$ & $0.00000 \mathrm{E}+00$ & $0.00000 \mathrm{E}+00$ & $4.79988 \mathrm{E}-13$ & $4.72635 \mathrm{E}-13$ & $5.24183 \mathrm{E}-13$ \\
\hline$D y-158$ & $0.00000 \mathrm{E}+00$ & $0.00000 \mathrm{E}+00$ & $0.00000 \mathrm{E}+00$ & $1.70080 \mathrm{E}-08$ & $1.67751 \mathrm{E}-08$ & $1.82661 \mathrm{E}-08$ \\
\hline$D y-160$ & $0.00000 \mathrm{E}+00$ & $0.00000 \mathrm{E}+00$ & $0.00000 \mathrm{E}+00$ & $2.09594 \mathrm{E}-01$ & $2.04839 \mathrm{E}-01$ & $2.21175 \mathrm{E}-01$ \\
\hline$D y-161$ & $0.00000 \mathrm{E}+00$ & $0.00000 \mathrm{E}+00$ & $0.00000 \mathrm{E}+00$ & $5.12699 \mathrm{E}-01$ & 4.91762E-01 & $5.20588 \mathrm{E}-01$ \\
\hline$D y-162$ & $0.00000 \mathrm{E}+00$ & $0.00000 \mathrm{E}+00$ & $0.00000 \mathrm{E}+00$ & $3.94381 \mathrm{E}-01$ & $3.86810 \mathrm{E}-01$ & 07595E-01 \\
\hline$D y-163$ & $0.00000 \mathrm{E}+00$ & $0.00000 \mathrm{E}+00$ & $0.00000 \mathrm{E}+00$ & $2.53825 \mathrm{E}-01$ & $2.44365 \mathrm{E}-01$ & $2.58660 \mathrm{E}-01$ \\
\hline$D y-164$ & $0.00000 \mathrm{E}+00$ & $0.00000 \mathrm{E}+00$ & $0.00000 \mathrm{E}+00$ & $4.05950 \mathrm{E}-02$ & $3.79241 \mathrm{E}-02$ & $.04034 \mathrm{E}-02$ \\
\hline Ho- 165 & $0.00000 \mathrm{E}+00$ & $0.00000 \mathrm{E}+00$ & $0.00000 \mathrm{E}+00$ & $8.89220 \mathrm{E}-02$ & $8.52888 \mathrm{E}-02$ & $.12269 \mathrm{E}-02$ \\
\hline Ho- $166 \mathrm{~m}$ & & $0.00000 \mathrm{E}+00$ & $0.00000 \mathrm{E}+00$ & 1441E-07 & $4.17966 \mathrm{E}-07$ & $66 \mathrm{E}-07$ \\
\hline$E r-162$ & $0.00000 \mathrm{E}+00$ & $0.00000 \mathrm{E}+00$ & $0.00000 \mathrm{E}+00$ & $6 \mathrm{E}-13$ & $4.10522 \mathrm{E}-13$ & $4 \mathrm{E}-13$ \\
\hline Er-164 & $0.00000 \mathrm{E}+00$ & $0.00000 \mathrm{E}+00$ & $0.00000 \mathrm{E}+00$ & $3.58595 \mathrm{E}-07$ & $3.54934 \mathrm{E}-07$ & 3.79445E-07 \\
\hline Er-166 & $0.00000 \mathrm{E}+00$ & $0.00000 \mathrm{E}+00$ & $0.00000 \mathrm{E}+00$ & $5152 \mathrm{E}-02$ & $1.88366 \mathrm{E}-02$ & $2.01322 \mathrm{E}-02$ \\
\hline Er-167 & $0.00000 \mathrm{E}+00$ & $0.00000 \mathrm{E}+00$ & $0.00000 \mathrm{E}+00$ & 4.96577E-04 & $4.94460 \mathrm{E}-04$ & $5.17979 \mathrm{E}-04$ \\
\hline$E r-168$ & $0.00000 \mathrm{E}+00$ & $0.00000 \mathrm{E}+00$ & $0.00000 \mathrm{E}+00$ & $2.93599 \mathrm{E}-03$ & $2.89093 \mathrm{E}-03$ & $3.08066 \mathrm{E}-03$ \\
\hline$E r-170$ & $0.00000 \mathrm{E}+00$ & $0.00000 \mathrm{E}+00$ & $0.00000 \mathrm{E}+00$ & $1.03538 \mathrm{E}-04$ & $1.03035 \mathrm{E}-04$ & $1.10455 \mathrm{E}-04$ \\
\hline Th-232 & $4.05956 \mathrm{E}+05$ & $4.06201 \mathrm{E}+05$ & $4.06200 \mathrm{E}+05$ & $3.88950 \mathrm{E}+05$ & $3.88973 \mathrm{E}+05$ & $3.88537 \mathrm{E}+05$ \\
\hline Th-233 & $0.00000 \mathrm{E}+00$ & $0.00000 \mathrm{E}+00$ & $0.00000 \mathrm{E}+00$ & $4.81700 \mathrm{E}-01$ & $4.95034 \mathrm{E}-01$ & 4.76749E-01 \\
\hline Th-234 & $0.00000 \mathrm{E}+00$ & $0.00000 \mathrm{E}+00$ & $0.00000 \mathrm{E}+00$ & $7.85341 \mathrm{E}-02$ & $8.26825 \mathrm{E}-02$ & $7.67482 \mathrm{E}-02$ \\
\hline$P a-231$ & $0.00000 \mathrm{E}+00$ & $0.00000 \mathrm{E}+00$ & $0.00000 \mathrm{E}+00$ & $1.91211 \mathrm{E}+00$ & $2.36480 \mathrm{E}+00$ & $2.46656 \mathrm{E}+00$ \\
\hline$P a-232$ & $0.00000 \mathrm{E}+00$ & $0.00000 \mathrm{E}+00$ & $0.00000 \mathrm{E}+00$ & $1.01709 \mathrm{E}-02$ & $1.26506 \mathrm{E}-02$ & $1.27491 \mathrm{E}-02$ \\
\hline$P a-233$ & $0.00000 \mathrm{E}+00$ & $0.00000 \mathrm{E}+00$ & $0.00000 \mathrm{E}+00$ & $8.35093 \mathrm{E}+02$ & $8.58132 \mathrm{E}+02$ & $.27220 \mathrm{E}+02$ \\
\hline$U-232$ & $0.00000 \mathrm{E}+00$ & $0.00000 \mathrm{E}+00$ & $0.00000 \mathrm{E}+00$ & $2.18659 \mathrm{E}+00$ & $2.69943 \mathrm{E}+00$ & $2.91788 \mathrm{E}+00$ \\
\hline$U-233$ & $0.00000 \mathrm{E}+00$ & $0.00000 \mathrm{E}+00$ & $0.00000 \mathrm{E}+00$ & $7.84522 \mathrm{E}+03$ & $7.91256 \mathrm{E}+03$ & $.10461 \mathrm{E}+03$ \\
\hline$U-234$ & $0.00000 \mathrm{E}+00$ & $0.00000 \mathrm{E}+00$ & $0.00000 \mathrm{E}+00$ & $9.95438 \mathrm{E}+02$ & $1.00084 \mathrm{E}+03$ & $1.03873 \mathrm{E}+03$ \\
\hline$U-235$ & $1.18213 \mathrm{E}+05$ & $1.18145 \mathrm{E}+05$ & $1.18146 \mathrm{E}+05$ & $2.85457 \mathrm{E}+04$ & $2.78704 \mathrm{E}+04$ & $2.92438 \mathrm{E}+04$ \\
\hline$U-236$ & $0.00000 \mathrm{E}+00$ & $0.00000 \mathrm{E}+00$ & $0.00000 \mathrm{E}+00$ & $1.48588 \mathrm{E}+04$ & $1.49514 \mathrm{E}+04$ & $1.49706 \mathrm{E}+04$ \\
\hline$U-237$ & $0.00000 \mathrm{E}+00$ & $0.00000 \mathrm{E}+00$ & $0.00000 \mathrm{E}+00$ & $2.59675 \mathrm{E}+01$ & $2.56368 \mathrm{E}+01$ & $2.48621 \mathrm{E}+01$ \\
\hline$U-238$ & $4.75831 \mathrm{E}+05$ & $4.75654 \mathrm{E}+05$ & $4.75655 \mathrm{E}+05$ & $4.46607 \mathrm{E}+05$ & $4.47433 \mathrm{E}+05$ & $4.45993 \mathrm{E}+05$ \\
\hline$U-239$ & $0.00000 \mathrm{E}+00$ & $0.00000 \mathrm{E}+00$ & $0.00000 \mathrm{E}+00$ & $8.41697 \mathrm{E}-01$ & $8.26451 \mathrm{E}-01$ & $7.98136 \mathrm{E}-01$ \\
\hline$U-240$ & $0.00000 \mathrm{E}+00$ & $0.00000 \mathrm{E}+00$ & $0.00000 \mathrm{E}+00$ & $1.61998 \mathrm{E}-04$ & $1.62923 \mathrm{E}-04$ & $1.51722 \mathrm{E}-04$ \\
\hline$U-241$ & $0.00000 \mathrm{E}+00$ & $0.00000 \mathrm{E}+00$ & $0.00000 \mathrm{E}+00$ & $5.99075 \mathrm{E}-10$ & $5.86197 \mathrm{E}-10$ & $5.29199 \mathrm{E}-10$ \\
\hline$N p-235$ & $0.00000 \mathrm{E}+00$ & $0.00000 \mathrm{E}+00$ & $0.00000 \mathrm{E}+00$ & $2.23221 \mathrm{E}-07$ & $2.53470 \mathrm{E}-07$ & $2.69712 \mathrm{E}-07$ \\
\hline$N p-236$ & $0.00000 \mathrm{E}+00$ & $0.00000 \mathrm{E}+00$ & $0.00000 \mathrm{E}+00$ & $2.40001 \mathrm{E}-04$ & $2.78581 \mathrm{E}-04$ & $2.93747 \mathrm{E}-04$ \\
\hline$N p-237$ & $0.00000 \mathrm{E}+00$ & $0.00000 \mathrm{E}+00$ & $0.00000 \mathrm{E}+00$ & $9.60667 \mathrm{E}+02$ & $9.37525 \mathrm{E}+02$ & $9.54347 \mathrm{E}+02$ \\
\hline$N p-238$ & $0.00000 \mathrm{E}+00$ & $0.00000 \mathrm{E}+00$ & $0.00000 \mathrm{E}+00$ & $3.49705 \mathrm{E}+00$ & $3.42229 \mathrm{E}+00$ & $3.36758 \mathrm{E}+00$ \\
\hline
\end{tabular}


Depletion Analysis of Modular High Temperature Gas-cooled Reactor Loaded with LEU/Thorium Fuel

February 2012

\begin{tabular}{|c|c|c|c|c|c|c|}
\hline \multirow[b]{2}{*}{ Nuclide } & \multicolumn{3}{|c|}{ Fresh Fuel } & \multicolumn{3}{|c|}{ Discharge } \\
\hline & Unit Cell & $\begin{array}{l}\text { Full Core } \\
(N O L B P)\end{array}$ & Full Core (3) & Unit Cell & $\begin{array}{l}\text { Full Core } \\
(N O L B P)\end{array}$ & Full Core (3) \\
\hline$N p-239$ & $0.00000 \mathrm{E}+00$ & $0.00000 \mathrm{E}+00$ & $0.00000 \mathrm{E}+00$ & $1.21618 \mathrm{E}+02$ & $1.19411 \mathrm{E}+02$ & $1.15325 \mathrm{E}+02$ \\
\hline$P u-236$ & $0.00000 \mathrm{E}+00$ & $0.00000 \mathrm{E}+00$ & $0.00000 \mathrm{E}+00$ & $2.63192 \mathrm{E}-06$ & $2.92301 \mathrm{E}-06$ & 3.19823E-06 \\
\hline$P u-237$ & $0.00000 \mathrm{E}+00$ & $0.00000 \mathrm{E}+00$ & $0.00000 \mathrm{E}+00$ & $4.74585 \mathrm{E}-05$ & $5.55725 \mathrm{E}-05$ & $5.91510 \mathrm{E}-05$ \\
\hline$P u-238$ & $0.00000 \mathrm{E}+00$ & $0.00000 \mathrm{E}+00$ & $0.00000 \mathrm{E}+00$ & $3.00022 \mathrm{E}+02$ & $2.87301 \mathrm{E}+02$ & $2.99767 \mathrm{E}+02$ \\
\hline$P u-239$ & $0.00000 \mathrm{E}+00$ & $0.00000 \mathrm{E}+00$ & $0.00000 \mathrm{E}+00$ & $3.66909 \mathrm{E}+03$ & $3.50890 \mathrm{E}+03$ & $3.55140 \mathrm{E}+03$ \\
\hline$P u-240$ & $0.00000 \mathrm{E}+00$ & $0.00000 \mathrm{E}+00$ & $0.00000 \mathrm{E}+00$ & $2.55300 \mathrm{E}+03$ & $2.61185 \mathrm{E}+03$ & $2.69440 \mathrm{E}+03$ \\
\hline$P u-241$ & $0.00000 \mathrm{E}+00$ & $0.00000 \mathrm{E}+00$ & $0.00000 \mathrm{E}+00$ & $1.75087 \mathrm{E}+03$ & $1.64411 \mathrm{E}+03$ & $1.72613 \mathrm{E}+03$ \\
\hline$P u-242$ & $0.00000 \mathrm{E}+00$ & $0.00000 \mathrm{E}+00$ & $0.00000 \mathrm{E}+00$ & $1.25683 \mathrm{E}+03$ & $1.19619 \mathrm{E}+03$ & $1.27890 \mathrm{E}+03$ \\
\hline$P u-243$ & $0.00000 \mathrm{E}+00$ & $0.00000 \mathrm{E}+00$ & $0.00000 \mathrm{E}+00$ & $2.76718 \mathrm{E}-01$ & $2.56329 \mathrm{E}-01$ & $2.65680 \mathrm{E}-01$ \\
\hline$P u-244$ & $0.00000 \mathrm{E}+00$ & $0.00000 \mathrm{E}+00$ & $0.00000 \mathrm{E}+00$ & $4.01355 \mathrm{E}-02$ & $3.59262 \mathrm{E}-02$ & $2.41276 \mathrm{E}-02$ \\
\hline Am-241 & $0.00000 \mathrm{E}+00$ & $0.00000 \mathrm{E}+00$ & $0.00000 \mathrm{E}+00$ & $3.75288 \mathrm{E}+01$ & $3.46617 \mathrm{E}+01$ & $3.87940 \mathrm{E}+01$ \\
\hline$A m-242$ & $0.00000 \mathrm{E}+00$ & $0.00000 \mathrm{E}+00$ & $0.00000 \mathrm{E}+00$ & $1.84887 \mathrm{E}-01$ & $1.72852 \mathrm{E}-01$ & $1.86752 \mathrm{E}-01$ \\
\hline$A m-243$ & $0.00000 \mathrm{E}+00$ & $0.00000 \mathrm{E}+00$ & $0.00000 \mathrm{E}+00$ & $1.88618 \mathrm{E}+02$ & $1.72203 \mathrm{E}+02$ & $1.87258 \mathrm{E}+02$ \\
\hline Am-244 & $0.00000 \mathrm{E}+00$ & $0.00000 \mathrm{E}+00$ & $0.00000 \mathrm{E}+00$ & $7.38466 \mathrm{E}-03$ & $6.57144 \mathrm{E}-03$ & $1.31635 \mathrm{E}-01$ \\
\hline$A m-242 m$ & $0.00000 \mathrm{E}+00$ & $0.00000 \mathrm{E}+00$ & $0.00000 \mathrm{E}+00$ & $7.35049 \mathrm{E}-01$ & $6.67488 \mathrm{E}-01$ & $7.51805 \mathrm{E}-01$ \\
\hline$A m-244 m$ & $0.00000 \mathrm{E}+00$ & $0.00000 \mathrm{E}+00$ & $0.00000 \mathrm{E}+00$ & $6.07026 \mathrm{E}-03$ & $5.40294 \mathrm{E}-03$ & 2.99699E-04 \\
\hline $\mathrm{Cm}-240$ & $0.00000 \mathrm{E}+00$ & $0.00000 \mathrm{E}+00$ & $0.00000 \mathrm{E}+00$ & $1.88621 \mathrm{E}-10$ & $2.09860 \mathrm{E}-10$ & $2.30279 \mathrm{E}-10$ \\
\hline Cm-241 & $0.00000 \mathrm{E}+00$ & $0.00000 \mathrm{E}+00$ & $0.00000 \mathrm{E}+00$ & $1.34583 \mathrm{E}-07$ & $1.53103 \mathrm{E}-07$ & $1.71688 \mathrm{E}-07$ \\
\hline Cm-242 & $0.00000 \mathrm{E}+00$ & $0.00000 \mathrm{E}+00$ & $0.00000 \mathrm{E}+00$ & $2.56440 \mathrm{E}+01$ & $2.38180 \mathrm{E}+01$ & $2.64380 \mathrm{E}+01$ \\
\hline Cm-243 & $0.00000 \mathrm{E}+00$ & $0.00000 \mathrm{E}+00$ & $0.00000 \mathrm{E}+00$ & 4.38814E-01 & 4.13732E-01 & 4.62786E-01 \\
\hline $\mathrm{Cm}-244$ & $0.00000 \mathrm{E}+00$ & $0.00000 \mathrm{E}+00$ & $0.00000 \mathrm{E}+00$ & $4.29192 \mathrm{E}+01$ & $3.74368 \mathrm{E}+01$ & $4.11564 \mathrm{E}+01$ \\
\hline Cm-245 & $0.00000 \mathrm{E}+00$ & $0.00000 \mathrm{E}+00$ & $0.00000 \mathrm{E}+00$ & $1.59383 \mathrm{E}+00$ & $1.35694 \mathrm{E}+00$ & $1.54133 \mathrm{E}+00$ \\
\hline
\end{tabular}

